\title{
Epigenetics, resilience and brain stimulation
}

Citation for published version (APA):

Nagy Adel, Y. (2020). Epigenetics, resilience and brain stimulation: advances in the mechanistic and therapeutic utility in patients with affective (PTSD and mood) disorders. [Doctoral Thesis, Maastricht University]. https://doi.org/10.26481/dis.20200123ny

Document status and date:

Published: 01/01/2020

DOI:

10.26481/dis.20200123ny

Document Version:

Publisher's PDF, also known as Version of record

\section{Please check the document version of this publication:}

- A submitted manuscript is the version of the article upon submission and before peer-review. There can be important differences between the submitted version and the official published version of record.

People interested in the research are advised to contact the author for the final version of the publication, or visit the DOI to the publisher's website.

- The final author version and the galley proof are versions of the publication after peer review.

- The final published version features the final layout of the paper including the volume, issue and page numbers.

Link to publication

\footnotetext{
General rights rights.

- You may freely distribute the URL identifying the publication in the public portal. please follow below link for the End User Agreement:

www.umlib.nl/taverne-license

Take down policy

If you believe that this document breaches copyright please contact us at:

repository@maastrichtuniversity.nl

providing details and we will investigate your claim.
}

Copyright and moral rights for the publications made accessible in the public portal are retained by the authors and/or other copyright owners and it is a condition of accessing publications that users recognise and abide by the legal requirements associated with these

- Users may download and print one copy of any publication from the public portal for the purpose of private study or research.

- You may not further distribute the material or use it for any profit-making activity or commercial gain

If the publication is distributed under the terms of Article $25 \mathrm{fa}$ of the Dutch Copyright Act, indicated by the "Taverne" license above, 


\section{EPIGENETICS, RESILIENCE, AND BRAIN STIMULATION}

ADVANCES IN THE MECHANISTIC AND

THERAPEUTIC UTILITY IN PATIENTS WITH AFFECTIVE DISORDERS

by

Nagy A. Youssef, MD 


\title{
Maastricht University
}

\section{EPIGENETICS, RESILIENCE, AND BRAIN STIMULATION: ADVANCES IN THE MECHANISTIC AND THERAPEUTIC UTILITY IN PATIENTS WITH AFFECTIVE (PTSD AND MOOD) DISORDERS}

\author{
DISSERTATION \\ to obtain the degree of Doctor at the Maastricht University, \\ on the authority of the Rector Magnificus, \\ Prof. Dr. Rianne M. Letschert \\ in accordance with the decision of the Board of Deans, \\ to be defended in public \\ on $23^{\text {rd }}$ day of January 2020, at 2:45 hours \\ by
}

Nagy A. Youssef 
Supervisor:

Prof. Dr. Bart P. F. Rutten

Co-supervisor:

Prof. Dr. Pascal Sienaert

Assessment Committee:

Prof. Dr. K.R.J. Schruers (Chairman)

Dr. B.M.G. Arts

Prof. Dr. F.P.M.L. Peeters

Prof. Dr. M. Van Den Bossche

Prof. Dr. Shaoyong Su 


\section{EPIGENETICS, RESILIENCE, AND BRAIN STIMULATION: ADVANCES IN THE MECHANISTIC AND THERAPEUTIC UTILITY IN PATIENTS WITH AFFECTIVE (PTSD AND MOOD) DISORDERS}

Nagy A. Youssef, MD 


\section{Promotor}

Prof. Dr. Bart P. F. Rutten

Maastricht University, The Netherlands

\section{Co-promotor}

Prof. Dr. Pascal Sienaert

University Psychiatric Center KU Leuven, Belgium

\section{Assessment Committee}

Prof. Dr. K.R.J. Schruers,

Maastricht University (Chairman)

Dr. B.M.G. Arts

Maastricht University, The Netherlands

Prof. Dr. F.P.M.L. Peeters

Maastricht University, The Netherlands

Prof. Dr. M. Van Den Bossche

University Psychiatric Center KU Leuven, Belgium

Prof. Dr. Shaoyong Su

Augusta University, United States of America 


\section{Table of Contents}

\section{PART I. EPIGENETICS \& RESILIENCE}

Chapter 1 General Introduction

Chapter 2 The Role of Epigenetics in Depression and Suicide: A Platform for GeneEnvironment Interactions

Chapter 3 The Effects of Trauma, With or Without PTSD, on the Transgenerational DNA Methylation Alterations in Human Offspring.

Chapter 4 Epigenome-Wide Association of PTSD From Heterogeneous Cohorts With a Common Multi-Site Analysis Pipeline 53

Chapter 5 A 3-Year Longitudinal Study Examining the Effect of Resilience on Suicidality in Veterans

Chapter 6 Racial/Ethnic Differences in the Association of Childhood Adversities With Depression and the Role of Resilience

Chapter 7 Moral Injury, Post-Traumatic Stress Disorder, and Religious Involvement in U.S. Veterans

\section{Part II. BRAIN STIMULATION}

Chapter 8 Relapse Prevention After Index Electroconvulsive Therapy in TreatmentResistant Depression

Chapter 9 Effects of Continuation Electroconvulsive Therapy on Quality of Life in Elderly Depressed Patients: A Randomized Clinical Trial

Chapter 10 Charting The ECT Course: Where Have We Been? Where Are We Headed?

Chapter 11 The Role of ECT in Posttraumatic Stress Disorder: A Systematic Review .... 196

Chapter 12 Feasibility, Safety, and Preliminary Efficacy of Low Amplitude Seizure Therapy (LAP-ST): A Proof of Concept Clinical Trial in Man

Chapter 13 General Discussion 235

Valorization of Knowledge 245 


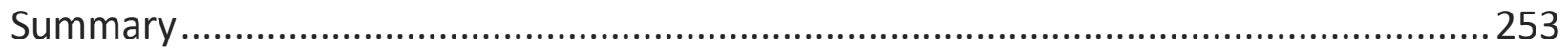

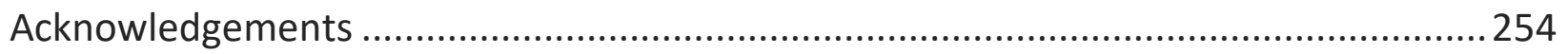

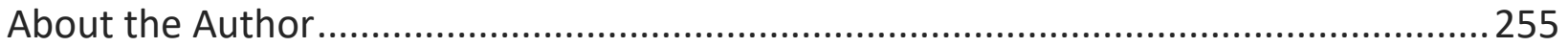


Chapter 1 General Introduction 


\section{INTRODUCTION}

Psychological adaptation reflects functional, cognitive and/or behavioral adjustment to contextual (environmental) changes that help the individual and comprises a key factor in psychological well-being. The mechanisms underlying adaptability to environmental challenges as well as the onset and course of mental disorders comprise biopsychosocial and existential factors. Based on advances in methodologies and conceptual ideas on how nature and nurture may interact, there is now ample opportunity to identify such factors and to advance our understanding. At the molecular biological level, epigenetic mechanisms may have an important role in the response to rapid environmental change and adaptation. For example, people who are resilient to disease development may have epigenetic makeup that fosters the adaptive processes to the environmental exposures during life. One of the newly evolving research and clinical tools that can be used to both modify neuronal function, adaptability, and neuroplasticity and to investigate underlying (biological) processes is brain stimulation (Bolwig \& Madsen, 2007; Joshi et al., 2016; Schloesser et al., 2015). Stimulation of the brain reflects exogenously induced or administered interventions and can thus be seen as an environmental exposure.

This thesis focuses and converges on two promising and rapidly evolving areas of neuroscience and psychiatry. These areas are epigenetics and brain stimulation. Research in both of these areas not only has the potential to transform our understanding of the onset and course of mental illness in high risk populations, but also in understanding resilience against mental illnesses in those who can "bounce back" from adversities with no development of mental illness (despite exposure to a similar environment).

Although the two main sections of this thesis, namely brain stimulation on one side and resilience and epigenetics on the other side, are not always related, some early studies in the literature have examined the relationship and interactions between both. For instance, there is evidence to suggest from Professor Rutten's laboratory that brain stimulation interventions may lead to epigenetic changes (de Jong JO et al., 2014). My hope is that this thesis will bring us a step forward to answering some of these questions in the two fields and spur interest in how both of these 2 fields can interact together and benefit from each other.

As for future directions after this thesis, I would like to investigate how brain stimulation interventions might improve resilience. An ECT study from our group, where I was the principal investigator, supports that patients who have undergone ECT for treatment-resistant depression also experienced improved resilience (publication pending). These relations might be mediated by epigenetics. I believe this to be important area to be explored further in the future as a follow up after my PhD.

On the other hand, epigenetics' signature may provide markers of treatment response for ECT or other brain stimulation interventions. The clinical implications of this are great. For instance, knowing beforehand through epigenetic testing who will respond to, or who will not respond to an acute course of ECT, can save a lot of suffering, cost, and time. This can individualize treatment to the right individual. 
Of course, more studies in these areas are needed in the future, and thus one of the goals of this thesis is to stimulate more studies about the interactions of the two areas. It is hoped that it spurs further studies in the field to contribute to our understanding of these two interesting fields and their relationships. Although this thesis will address these two areas separately, given my interest in both, the work in this thesis will act as preparation and a sounding board for me for the next steps after my PhD, which is to examine and perform studies that involve interactions between both areas to further understand the above relationships.

The first section of this thesis focuses on epigenetics as one possible molecular mechanism underlying risk and resilience to psychological trauma in relation to subsequent development of affective disorders such as PTSD and depression.

Several studies in the literature have described the relationship between resilience and epigenetics, where epigenetic alterations might cause certain individuals to be more resilient to the effects of trauma and depression than others. Resilience does not only have a psychological contribution but also a biological contribution, which probably has an epigenetic basis.

The second section of this thesis will focus on electroconvulsive therapy (ECT). Of the brain stimulation interventions, ECT is considered to be not only the oldest (with much improved modern techniques for application), but also the most effective treatment known to man for several psychiatric disorders, especially mood disorders (Greenberg \& Kellner, 2005; Lin, Huang, \& Chen, 2018). Thus, ECT is considered the prototype of brain stimulation techniques and will be the focus of this thesis.

Moreover, ECT has the most research support among neuromodulation devices. In this thesis, I will also shed some light on cutting-edge research developed to mitigate (or avoid) cognitive side effects of ECT and how modern modification has markedly improved the safety and tolerability of ECT.

\section{General introduction on epigenetics and its role in mental disorders:}

The field of epigenetics may offer useful insights into differential susceptibility of risk to develop psychopathology as well as resilience. According to Goldberg et al., epigenetics is the study of functionally stable and (ideally) heritable changes in gene expression or cellular phenotype that occurs without changes in base pairing (Goldberg, Allis, \& Bernstein, 2007). The National Institutes of Health in the United States provides a broader definition, wherein their 2009 epigenomics initiative stated that "epigenetics refers to both heritable changes in gene activity and expression (in the progeny of cells or of individuals) and also stable, long-term alterations in the transcriptional potential of a cell that are not necessarily heritable" (National Advisory Mental Health Council Workgroup on Genomics, 2018).

Thus, epigenetics involves functional changes to the gene without sequence changes. The best-studied epigenetic mechanism so far is DNA methylation. DNA methylation involves the 
attachment of methyl groups to the DNA molecule. When methyl groups are attached to the promoter region, they typically act to repress gene transcription.

Certain DNA methylation alterations have been associated with several mental phenotypes. For instance, we found an association between hypermethylation of either Brainderived neurotrophic factor (BDNF) promoter or tropomycin receptor kinase B (TrkB) and suicide (Lockwood, Su, \& Youssef, 2015).

Interactions between the environment and biological background of an individual during the onset and course of mental disorders may be better understood in the lens of epigenetics. This is because epigenetics may be an important link that explains how the environment (trauma or stressor) interfaces with the biological machinery. The exposure to psychological trauma has been shown to increase risk of the onset of PTSD and depression (Hovens, Giltay, Spinhoven, van Hemert, \& Penninx, 2015; Hovens et al., 2012; Youssef et al., 2017; Youssef et al., 2013). Also, chronic severe stress has been observed to exacerbate or induce an episode of depression. Childhood trauma has even been associated with the development of physical illnesses such as cardiometabolic disease in adulthood, and evidence suggests the involvement of an epigenetic mechanism (Hao, Youssef, Davis, \& Su, 2018).

An important question that frequently arises is: Why do some people develop mental disorders after experiencing a traumatic event, while others do not? Possible explanations could be an underlying genetic or epigenetic risk in those who are more prone to develop PTSD or depression. Similarly, those who are resilient to disease development may have some epigenetic makeup that facilitates the adaptive processes.

In this example, PTSD has long been established to be due to exposure to trauma (trauma is even the cardinal criterion for PTSD; without it PTSD cannot be diagnosed). Thus, it has been assumed that only environmental factors, namely trauma, contribute to the development of PTSD. However, emerging data show that: Although trauma is necessary, it is not sufficient for PTSD development. A range of studies has now estimated that PTSD shows considerable heritability component that is between $30 \%$ and $70 \%$ in twin studies (Sartor et al., 2012; Stein, Jang, Taylor, Vernon, \& Livesley, 2002; True et al., 1993; Xian et al., 2000). This indicates the role of heritable factors related to either DNA structure or function. Then again, many decades of genetic studies of both candidate gene and genome-wide association studies (GWAS) have provided many interesting and promising findings, yet so far no robust structural genetic variants for PTSD have been identified (Logue et al., 2015). This risk is unlikely to be fully explained by only structural genetics (Ratanatharathorn et al., 2017). Thus, the field of epigenetics could offer great insights into differential susceptibility of risk to develop psychopathology.

\section{Epigenetics, gene expression, and cell type specificity:}


Although all of our cells contain the same DNA, the cell types and functions vary greatly, resulting in different organs. This is due to both qualitative and quantitative differences in their gene expression.

The transfer of genetic information from the DNA to the messenger RNA (mRNA), (usually resulting in the subsequent protein formation or modification) is done through transcription then translation into proteins.

Epigenetic mechanisms, including DNA methylation, among others, influence gene expression mainly at the level of transcription. However, translation may also be regulated epigenetically.

Epigenetic response to environmental change and epigenetic mediation of neuronal plasticity:

Epigenetic mechanisms may have an important role in the response to rapid environmental change and adaptation (Schrey et al., 2016). This may involve behavioral or physiological adaptation. However, certain environmental stressors and trauma might lead to dysfunctional responses that lead to symptoms and disorders in susceptible individuals, while not in others.

The epigenetic alteration in the response to rapid environmental changes also requires an interface between epigenetics and neuronal plasticity. The interaction between epigenetics and plasticity can be in the form of either functional (physiological) or structural changes in the neurons in response to internal or external stimuli and stressors. If the adaption is dysfunctional, or the system is overwhelmed and unable to adapt altogether, this can lead to disease development. Likewise, in other individuals, the physiological changes lead to successful functional adaptation (and possibly structural), and thus amount to rapid recovery from trauma or resilience against trauma or stressors in the face of the same type and intensity of the trauma/stressor.

\section{General introduction to brain stimulation and approaches for stimulation:}

As for the field of brain stimulation, it commonly involves either electric or magnetic stimulation of particular brain circuit(s) or region(s) to modulate (stimulation, inhibition, etc.) neuronal activity in specific circuit(s) or region(s) of the brain. Such brain stimulation interventions can in principle be used to modulate neuronal activity either for therapeutic reasons as commonly used clinically (as well as for research purposes to develop therapeutic interventions), or as a probe in order to identify certain conditions or states (as a biomarker). The use of brain stimulation modalities as probes/biomarkers is now mostly confined to research and is not yet in clinical use. Brain stimulation modalities involve transcranial, noninvasive stimulation, but can also be intracranial as in the case of deep brain stimulation (DBS). ECT is the 
oldest and most effective method of noninvasive brain stimulation that is commonly used in clinical practice. ECT is a prototype of brain stimulation and will be the focus of the brain stimulation section of this thesis.

Brain stimulation comprises the administration of a direct, specific environmental stimulus that can modify neuronal functioning, neuroplasticity, and possibly epigenetics. Indeed, not only can electric or magnetic brain stimulation modify neuronal functioning for fostering treatment and recovery, but it can also be considered as a tool in boosting resilience (Bolwig \& Madsen, 2007; Joshi et al., 2016; Schloesser et al., 2015).

Brain stimulation can provide a focused environmental stimulus to certain regions of the brain, and some have even considered brain stimulatory approaches for the restoration of healthy states as well as for the promotion of healing and recovery (Veena et al., 2009).

Environmental enrichment through offering enhanced positive stimulation to an individual has been suggested to improve brain function. Environmental enrichment has also been connected to structural neuronal and synaptic formation (Fan, Liu, Weinstein, Fike, \& Liu, 2007; Veena et al., 2009), prevention (and even reversal) of neuronal loss in the hippocampus (after chronic stress) by inducing adult hippocampal regeneration of neurons (Veena et al., 2009), and possibly hippocampal neurogenesis (Cao et al., 2018; Joshi et al., 2016; Schloesser et al., 2015; Tendolkar et al., 2013). This has also been observed in studies using other brain stimulation modalities such as vagal nerve stimulation, suggesting a "modulatory effect on hippocampal plasticity" as mentioned by the Perini and colleagues' study (Perini et al., 2017).

\section{What main questions have arisen in the fields of epigenetics and brain stimulation?}

There are/remain a lot of unanswered questions, both in the field of epigenetics and brain stimulation. Some of these important questions are as follows:

- Is there evidence that depression is associated with epigenetic alterations? Similarly, is there evidence that epigenetic alterations are associated with suicide or suicidality? Are there transgenerational effects of trauma and/or PTSD, or does trauma only affect the person exposed to it?

Answers to these questions and many more in epigenetics, as well as replication of existing studies in a larger dataset and in diverse populations, will be improved by data sharing between many centers. Sharing data will generate a substantial sample size and meaningful strong significances in genome-wide studies (Ratanatharathorn et al., 2017), as indicated also by the National Advisory Mental Health Council Workgroup on Genomics, 2018 (NAMHC) (National Advisory Mental Health Council Workgroup on Genomics, 2018). Thus, it is key to ask: 
- Is it feasible to assemble an epigenetics consortium to share and pool data for generating a large sample size (to increase the power of detection) in epigenetic studies?

Other important questions on the clinical side are:

- What is the meaning of a certain traumatic event (e.g. combat trauma) to the specific individual? Does the moral code and/or their spiritual or religious beliefs color the clinical presentation in that it has an effect on development or lack of development of psychopathology?

- What is the relationship between moral injury and severity of PTSD symptoms in U.S. military veterans? Are there mediating and moderating effects of religiosity on the relationship between moral injury and PTSD severity?

Moral injury has been defined by Litz and colleagues as "perpetrating, failing to prevent, bearing witness to, or learning about acts that transgress deeply held moral beliefs." Moral injury, a construct that is different from PTSD, has been described after traumatic experiences during wartime (Litz et al., 2009; Nash \& Litz, 2013; Shay, 1994) and can co-occur with PTSD.

Moral injury results from experiences that violate deeply held moral beliefs and values, which then arouse shame, guilt, and existential questioning. Moral injury symptoms include both psychological and spiritual symptoms (loss of meaning and hope, spiritual struggles, loss of faith) (Koenig et al., 2017). Treatments targeting moral injury (both spiritual and secular) have been shown to reduce PTSD symptoms (Gray et al., 2012; Ogden et al., 2011).

Other related important questions in this clinical area include:

- Is there a dose-response relationship between trauma and depressive symptoms?

- Is early childhood trauma effect different or similar across difference races? For instance, does early childhood trauma affect European Americans (EA) and African Americans (AA) in a similar fashion?

- Does resilience mitigate the effect of early childhood trauma on depressive symptoms?

- What is the effect of resilience on suicidality? More specifically, would higher resilience decrease the risk of suicide, thus have a protective effect against suicidality?

Some overarching and important questions in the field of brain stimulation are as follows:

- What are the best strategies to avoid relapse and recurrence of depressive symptoms after an acute ECT course? 
- Does continuation ECT plus medications have superior impact on quality of life after remission of a depressive episode with an acute course of ECT compared to continuation medications alone?

- What is the current state of knowledge in the field of ECT regarding indications, patient selection, choice of electrode placement, and stimulus parameters?

- What are the future directions of research in the field of ECT including biomarkers and new techniques for delivering ECT?

- Is there preliminary human data that ECT can also be effective for PTSD, especially treatment-resistant PTSD? What is the feasibility (especially for the ability to consistently induce seizures) and safety of using a low current amplitude 0.5-0.6A (Low Pulse Amplitude Seizure Therapy or LAP-ST) for treatment of depressive episodes? Is there preliminary evidence that LAP-ST has fewer cognitive adverse effects compared to standard ECT while retaining efficacy?

\section{Overview of thesis}

This thesis attempts to answer most of these questions both in the fields of epigenetics as well as brain stimulation. It addresses these fields into the context of two main disease classifications (both with significant genetic-environmental (GXE) interaction) namely mood disorders and PTSD. Thus, the research compiled in this thesis can be divided in two main sections. The first section focuses on 'Epigenetics and Resilience,' and the second section focuses on 'Brain Stimulation' with ECT as the prototype for brain stimulation treatments for the purpose of this thesis. The thesis will also introduce a novel form of ECT, namely Low Amplitude Seizure Therapy (LAP-ST).

The first section focuses on epigenetics and resilience and starts with Chapter 2, which defines epigenetics, and critically reviews the available studies on the association between epigenetics and depression.

In order to examine the available literature on the effects of individual trauma exposure and PTSD on the offspring, and if there is a probability of transgenerational transmission via epigenetic alterations, Chapter $\mathbf{3}$ critically reviews the human literature that discusses and studies the possible role of epigenetic methylation transmission from one generation to the next. The chapter also concludes with a discussion of the challenges that the field of epigenetics faces and provides guidelines for improving the quality of future epigenetic research.

In order to examine the feasibility to assemble an epigenetics consortium to share and pool data for generating a large sample size, Chapter 4 describes our efforts in assembling a consortium to develop a common pipeline for Epigenome-Wide Association of PTSD collected 
from many centers to increase the number of participants and statistical power needed for epigenetic research. These data from different cohorts/centers will have a Common Multi-Site Analysis Pipeline.

Since epigenetics may also play a role in resilience, the following chapters (Chapters 5, 6, and 7) switch gears from the molecular level to the clinical level by presenting studies of resilience to mental problems. Given that spirituality has been described as one of the domains for the Conner Davidson Resilience Scale (CD-RISC) and that moral values and meaning of life (Snijders et al., 2018) have been suggested to influence resilience, we examine spirituality and moral injury in veterans with PTSD.

In order to examine the effect of resilience on suicidality and if higher resilience decreases the risk of suicide, Chapter 5 presents data from our 3-year longitudinal study of 178 veterans that examines this relationship.

In order to examine whether there is a dose-response relationship between trauma and depressive symptoms, whether early trauma affects European Americans and African Americans in a similar fashion, and whether resilience mitigates the effect of trauma on depression, Chapter 6 presents our study of 413 participants to answer these questions.

Chapter 7 describes our cross-sectional study of 120 U.S. combat veterans in an effort to answer inquiries related to 1) the meaning of combat trauma to a specific individual based on their moral code and/or spiritual or religious beliefs, 2) the relationship between moral injury and severity of PTSD symptoms in U.S. military veterans, and 3) any mediating and moderating effects of religiosity on the relationship between moral injury and PTSD severity.

Section two of the thesis focuses on ECT. Treatment-resistant depression (TRD) is one of the most common indications for ECT. Thus, the section starts with Chapter 8, which presents a critical systematic review of the literature to examine the best way to prevent relapse in TRD after remission of an episode, and whether ECT alone, pharmacology alone, or combined ECT and pharmacology would be the best way to prevent relapse after an acute course of ECT for TRD.

In those who had full remission of depressive symptoms after an acute course of ECT, the question then becomes: Does continuation of ECT plus medications have a superior impact on quality of life compared to continuation of medications alone? Thus, Chapter 9 presents our randomized multicenter clinical trial on the effects of continuation of ECT plus medications versus continuation of medication alone on improving the quality in a total sample of 120 patients to address this question.

In order to briefly overview the literature on the current knowledge of ECT and the future direction of ECT, Chapter 10 discusses the achievements so far in ECT, including current knowledge on indication, patient selection, choice of electrode placement and stimulus parameters, augmentation with pharmacotherapy, and the use of continuation/maintenance treatment. Then, it discusses cutting-edge research in the field including research into 
biomarkers and neuroplasticity related to ECT response, as well as new, investigational methods of ECT delivery. Then, it charts the sea of future research of "where we are headed."

In order to examine any available evidence that ECT might be of help in PTSD (especially for treatment-resistant cases) and if this warrants a definitive randomized clinical trial, Chapter 11 critically reviews the literature of the potential use of ECT for PTSD by reviewing the emerging literature and providing directions for future research.

Although ECT is the most effective treatment for TRD, cognitive (including memory) side effects can both be burdensome to some patients who were treated with ECT, and may discourage others who desperately need this life-saving intervention from receiving it. Thus, in order to find ways to avoid (or minimize) the cognitive side effects, we examine a more focal form of stimulation: Low Pulse Amplitude Seizure Therapy (LAP-ST) for treatment of depressive episodes. In this clinical trial, we specifically examine the feasibility and any preliminary evidence that it has less cognitive adverse effects compared to standard ECT. Thus, Chapter 12 presents this investigational technique and the first open-label proof of concept (POC) clinical trial using LAP-ST to examine this technique and answer these questions. Chapter 13 is a "Discussion" of the concepts presented in this thesis, including the summary, limitations, and recommended future research directions. 


\section{REFERENCES}

Bolwig, T. G., \& Madsen, T. M. (2007). Electroconvulsive therapy in melancholia: the role of hippocampal neurogenesis. Acta Psychiatr Scand Suppl(433), 130-135. doi:10.1111/j.1600-0447.2007.00971.x

Cao, B., Luo, Q., Fu, Y., Du, L., Qiu, T., Yang, X., ... Qiu, H. (2018). Predicting individual responses to the electroconvulsive therapy with hippocampal subfield volumes in major depression disorder. Sci Rep, 8(1), 5434. doi:10.1038/s41598-018-23685-9

de Jong JO, Arts B, Boks MP, Sienaert P, van den Hove DL, Kenis G, van Os J, Rutten BP. Epigenetic effects of electroconvulsive seizures. J ECT. 2014 Jun;30(2):152-9. doi: 10.1097/YCT.0000000000000141.

Fan, Y., Liu, Z., Weinstein, P. R., Fike, J. R., \& Liu, J. (2007). Environmental enrichment enhances neurogenesis and improves functional outcome after cranial irradiation. Eur J Neurosci, 25(1), 38-46. doi:10.1111/j.1460-9568.2006.05269.x

Goldberg, A. D., Allis, C. D., \& Bernstein, E. (2007). Epigenetics: a landscape takes shape. Cell, 128(4), 635-638. doi:10.1016/j.cell.2007.02.006

Gray, M. J., Schorr, Y., Nash, W., Lebowitz, L., Amidon, A., Lansing, A., . . Litz, B. T. (2012). Adaptive disclosure: an open trial of a novel exposure-based intervention for service members with combat-related psychological stress injuries. Behav Ther, 43(2), 407-415. doi:10.1016/j.beth.2011.09.001

Greenberg, R. M., \& Kellner, C. H. (2005). Electroconvulsive therapy: a selected review. Am J Geriatr Psychiatry, 13(4), 268-281. doi:10.1176/appi.ajgp.13.4.268

Hao, G., Youssef, N. A., Davis, C. L., \& Su, S. (2018). The role of DNA methylation in the association between childhood adversity and cardiometabolic disease. Int J Cardiol, 255, 168-174. doi:10.1016/j.ijcard.2017.12.063

Hovens, J. G., Giltay, E. J., Spinhoven, P., van Hemert, A. M., \& Penninx, B. W. (2015). Impact of childhood life events and childhood trauma on the onset and recurrence of depressive and anxiety disorders. J Clin Psychiatry, 76(7), 931-938. doi:10.4088/JCP.14m09135

Hovens, J. G., Giltay, E. J., Wiersma, J. E., Spinhoven, P., Penninx, B. W., \& Zitman, F. G. (2012). Impact of childhood life events and trauma on the course of depressive and anxiety disorders. Acta Psychiatr Scand, 126(3), 198-207. doi:10.1111/j.1600-0447.2011.01828.x

Joshi, S. H., Espinoza, R. T., Pirnia, T., Shi, J., Wang, Y., Ayers, B., . . Narr, K. L. (2016). Structural plasticity of the hippocampus and amygdala induced by electroconvulsive therapy in major depression. Biol Psychiatry, 79(4), 282-292. doi:10.1016/j.biopsych.2015.02.029

Koenig, H. G., Boucher, N. A., Oliver, R. J. P., Youssef, N., Mooney, S. R., Currier, J. M., \& Pearce, M. (2017). Rationale for spiritually oriented cognitive processing therapy for moral injury in active duty military and veterans with posttraumatic stress disorder. Journal of Nervous and Mental Disease, 205(2), 147-153. doi:10.1097/NMD.0000000000000554 
Lin, C. H., Huang, C. J., \& Chen, C. C. (2018). ECT Has Greater Efficacy Than Fluoxetine in Alleviating the Burden of Illness for Patients with Major Depressive Disorder: A Taiwanese Pooled Analysis. Int J Neuropsychopharmacol, 21(1), 63-72. doi:10.1093/ijnp/pyx114

Litz, B. T., Stein, N., Delaney, E., Lebowitz, L., Nash, W. P., Silva, C., \& Maguen, S. (2009). Moral injury and moral repair in war veterans: a preliminary model and intervention strategy. Clin Psychol Rev, 29(8), 695-706. doi:10.1016/j.cpr.2009.07.003

Lockwood, L. E., Su, S., \& Youssef, N. A. (2015). The role of epigenetics in depression and suicide: a platform for gene-environment interactions. Psychiatry Res, 228(3), 235-242. doi:10.1016/j.psychres.2015.05.071

Logue, M. W., Amstadter, A. B., Baker, D. G., Duncan, L., Koenen, K. C., Liberzon, I., . . Ressler, K. J. (2015). The Psychiatric Genomics Consortium Posttraumatic Stress Disorder Workgroup: posttraumatic stress disorder enters the age of large-scale genomic collaboration. Neuropsychopharmacology, 40(10), 2287-2297. doi:10.1038/npp.2015.118

Nash, W. P., \& Litz, B. T. (2013). Moral injury: a mechanism for war-related psychological trauma in military family members. Clin Child Fam Psychol Rev, 16(4), 365-375. doi:10.1007/s10567-013-0146-y

National Advisory Mental Health Council Workgroup on Genomics. (2018). Opportunities and Challenges of Psychiatric Genetics. Retrieved from Bethesda, MD; National Advisory Mental Health Council (NAMHC), National Institutes of Health (NIH): https://www.nimh.nih.gov/about/advisory-boards-and-groups/namhc/reports/reportof-the-national-advisory-mental-health-council-workgroup-on-genomics.shtml

Ogden, H., Harris, J. I., Erbes, C. R., Engdahl, B. E., Olson, R. H., Winskowski, A. M., \& McMahill, J. (2011). Religious functioning and trauma outcomes among combat veterans. Counseling et spiritualité, 30(1), 71-89.

Perini, G. I., Toffanin, T., Pigato, G., Ferri, G., Follador, H., Zonta, F., . . D'Avella, D. (2017). Hippocampal gray volumes increase in treatment-resistant depression responding to vagus nerve stimulation. J ect, 33(3), 160-166. doi:10.1097/yct.0000000000000424

Ratanatharathorn, A., Boks, M. P., Maihofer, A. X., Aiello, A. E., Amstadter, A. B., Ashley-Koch, A. E., . . . Smith, A. K. (2017). Epigenome-wide association of PTSD from heterogeneous cohorts with a common multi-site analysis pipeline. Am J Med Genet B Neuropsychiatr Genet, 174(6), 619-630. doi:10.1002/ajmg.b.32568

Sartor, C. E., Grant, J. D., Lynskey, M. T., McCutcheon, V. V., Waldron, M., Statham, D. J., . . . Nelson, E. C. (2012). Common heritable contributions to low-risk trauma, high-risk trauma, posttraumatic stress disorder, and major depression. Archives of General Psychiatry, 69(3), 293-299. doi:10.1001/archgenpsychiatry.2011.1385

Schloesser, R. J., Orvoen, S., Jimenez, D. V., Hardy, N. F., Maynard, K. R., Sukumar, M., ... . Martinowich, K. (2015). Antidepressant-like effects of electroconvulsive seizures require 
adult neurogenesis in a neuroendocrine model of depression. Brain Stimul, 8(5), 862867. doi:10.1016/j.brs.2015.05.011

Schrey, A. W., Robbins, T. R., Lee, J., Dukes, D. W., Jr., Ragsdale, A. K., Thawley, C. J., \& Langkilde, T. (2016). Epigenetic response to environmental change: DNA methylation varies with invasion status. Environ Epigenet, 2(2), dvw008. doi:10.1093/eep/dvw008

Shay, J. (1994). Achilles in Vietnam: combat trauma and the undoing of character. New York: Atheneum.

Snijders, C., Pries, L., Sgammeglia, N., Youssef, N., de Nijs, L., Guloksuz, S., \& Rutten, B. (2018). Resilience against traumatic stress: current developments and future directions. Frontiers in Psychiatry, 9(676). doi:10.3389/fpsyt.2018.00676

Stein, M. B., Jang, K. L., Taylor, S., Vernon, P. A., \& Livesley, W. J. (2002). Genetic and environmental influences on trauma exposure and posttraumatic stress disorder symptoms: a twin study. Am J Psychiatry, 159(10), 1675-1681. doi:10.1176/appi.ajp.159.10.1675

Tendolkar, I., van Beek, M., van Oostrom, I., Mulder, M., Janzing, J., Voshaar, R. O., \& van Eijndhoven, P. (2013). Electroconvulsive therapy increases hippocampal and amygdala volume in therapy refractory depression: a longitudinal pilot study. Psychiatry Res, 214(3), 197-203. doi:10.1016/j.pscychresns.2013.09.004

True, W. R., Rice, J., Eisen, S. A., Heath, A. C., Goldberg, J., Lyons, M. J., \& Nowak, J. (1993). A twin study of genetic and environmental contributions to liability for posttraumatic stress symptoms. Arch Gen Psychiatry, 50(4), 257-264. doi:10.1001/archpsyc.1993.01820160019002

Veena, J., Srikumar, B. N., Mahati, K., Bhagya, V., Raju, T. R., \& Shankaranarayana Rao, B. S. (2009). Enriched environment restores hippocampal cell proliferation and ameliorates cognitive deficits in chronically stressed rats. J Neurosci Res, 87(4), 831-843. doi:10.1002/jnr.21907

Xian, H., Chantarujikapong, S. I., Scherrer, J. F., Eisen, S. A., Lyons, M. J., Goldberg, J., ... True, W. R. (2000). Genetic and environmental influences on posttraumatic stress disorder, alcohol and drug dependence in twin pairs. Drug \& Alcohol Dependence, 61(1), 95-102. doi:10.1016/S0376-8716(00)00127-7

Youssef, N. A., Belew, D., Hao, G., Wang, X., Treiber, F. A., Stefanek, M., . . Su, S. (2017). Racial/ethnic differences in the association of childhood adversities with depression and the role of resilience. J Affect Disord, 208, 577-581. doi:10.1016/j.jad.2016.10.024

Youssef, N. A., Green, K. T., Dedert, E. A., Hertzberg, J. S., Calhoun, P. S., Dennis, M. F., \& Beckham, J. C. (2013). Exploration of the influence of childhood trauma, combat exposure, and the resilience construct on depression and suicidal ideation among U.S. Iraq/Afghanistan era military personnel and veterans. Arch Suicide Res, 17(2), 106-122. doi:10.1080/13811118.2013.776445 


\section{Chapter 2 The Role of Epigenetics in Depression and Suicide: A Platform for Gene-Environment Interactions}

Laura E. Lockwood ${ }^{1}$, Shaoyong Su ${ }^{2}$, Nagy A. Youssef ${ }^{1,3}$

${ }^{1}$ Department of Psychiatry \& Health Behavior, The Medical College of Georgia, Augusta University, USA.

${ }^{2}$ Georgia Prevention Institute, Medical College of Georgia, Augusta University, USA.

${ }^{3}$ Charlie Norwood VA Medical Center, Augusta, GA, USA.

Published as: Lockwood LE, SU S, Youssef NA. The role of epigenetics in depression and suicide: A platform for gene-environment interactions. Psychiatry Res. 2015 Aug 30;228(3):235-42. doi: 10.1016/j.psychres.2015.05.071. Epub 2015 Jun 27. Review. PubMed PMID: 26163724. https://www.sciencedirect.com/science/article/pii/S0165178115003832?via\%3Dihub 


\section{Abstract}

Epigenetics involves functional modifications of genes that are affected by environmental factors. The aim of this paper is to explore if an association exists between epigenetics and depression and/or suicide. Medline/PubMed searches were performed using both Medical Subject Heading (MeSH) and Non-MeSH terms. Based on pre-specified terms and inclusion criteria, sixteen studies met inclusion criteria by 2 independent reviewers. Epigenetic changes seem to be important in both depression and suicide. All of the studies reviewed herein found significant epigenetic changes associated with depression and suicide except for two. Several studies showed that hypermethylation of BDNF is involved in suicide. TrkB hypermethylation was also shown to be associated with suicide by several studies, specifically in Brodmann's Areas (BA) 8 and 9. Future research is needed in a larger sample to further characterize these changes.

\section{Keywords}

Epigenetics, Depression, Suicide, Suicidality, Genes, Methylation of Genes, Gene-Environment Interactions 


\section{INTRODUCTION}

Epigenetics involves functional modifications of genes that are affected by environmental factors. Goldberg et al (2007) defines epigenetics in a broad sense as a "bridge between genotype and phenotype." They also define it more specifically as "the study of any potentially stable and, ideally, heritable changes in gene expression or cellular phenotype that occurs without changes in Watson-Crick base-pairing of DNA" (Goldberg, Allis, \& Bernstein, 2007). Epigenetics involves functional changes to the genome that may result in it being "turned off" or "turned on," and does not involve a change in the nucleotide sequence or structure. "Epigenetic markers on DNA can be influenced by environmental forces, such as smoking, diet, and pollutants" (Yan, 5 Mar 2010) or even medications and other therapeutic interventions. "In contrast to the genetic sequence, which is determined by inheritance and is virtually identical in all tissues, the epigenetic pattern varies from cell type to cell type and is potentially dynamic throughout life. It is postulated here that different environmental exposures. . could impact epigenetic patterns, with important implications for mental health" (McGowan \& Szyf, 2010). In contrast to genetic sequence alterations, "epigenetic alterations are potentially reversible" (McGowan \& Szyf, 2010).

Depression is a likely candidate for a disease that would have epigenetic mechanisms associated with it, as there has long been a recognized connection between environmental factors and depression (Wankerl et al., 2014). If an association is found between epigenetics and depression and suicide, this could shed light on suicide prevention efforts as well as identification of biomarkers for depression that could possibly help with disease stratification.

The aim of this paper is to perform a systematic review of the literature related to the role of epigenetics and depression and suicide. We hypothesized that an association does exist.

\section{METHODS}

Inclusion criteria for this systematic review were studies with original data which were written in English. Exclusion criteria were review papers and papers written in languages other than English.

A search of Medline/PubMed was performed using Medical Subject Heading (MeSH) Database for articles from inception to February 23, 2015. The search terms were "Epigenomics" [Mesh] and "Depressive Disorder" [Mesh]. Of the 11 papers produced by the search, 9 were excluded because they were review papers, and one was excluded due to being a letter. The remaining study was reviewed herein.

Another search was performed using PubMed MeSH from inception to February 23, 2015. The search terms were "Suicide" [Mesh] and "Epigenomics" [Mesh]. This search yielded 9 papers, 3 of which met our inclusion criteria. The remaining 6 papers were excluded due to being review articles.

Non-MeSH searches were also performed, using the terms "epigenetics depression," "epigenetics suicide," "epigenetics suicidal ideation," "epigenomics depression," "epigenomics suicide," and "epigenomics suicidal ideation." When searching "epigenetics depression," 3 additional papers were found; 2 more were found when searching "epigenetics suicide"; 1 more 
was found when searching "epigenetics suicidal ideation"; and no additional papers were found when searching "epigenomics depression," "epigenomics suicide," or "epigenomics suicidal ideation."

A search was conducted using the terms "(epigenetics OR epigenomics) AND (depressive disorder OR depression)." This search resulted in 164 papers. Of these, 65 were excluded due to being reviews; 1 was excluded due to being in a foreign language; 11 were excluded due to being both reviews and in a foreign language; 70 did not meet the objectives of the review, and 6 were letters. Five of the papers had already been included from other searches, and 5 more papers were found to add to the review.

A similar search of "(epigenetics OR epigenomics) AND (suicide OR suicidal ideation)" was performed, which yielded 28 papers. Of these, 9 were excluded due to being reviews; 1 was excluded due to being in a foreign language; 1 was a letter, and 83 were excluded due to not meeting the objectives of the review. Eight of the papers had already been included in the review from prior searches, and 1 new paper was added. Of note, both human and animal studies were included. See Figure 1 for more details.

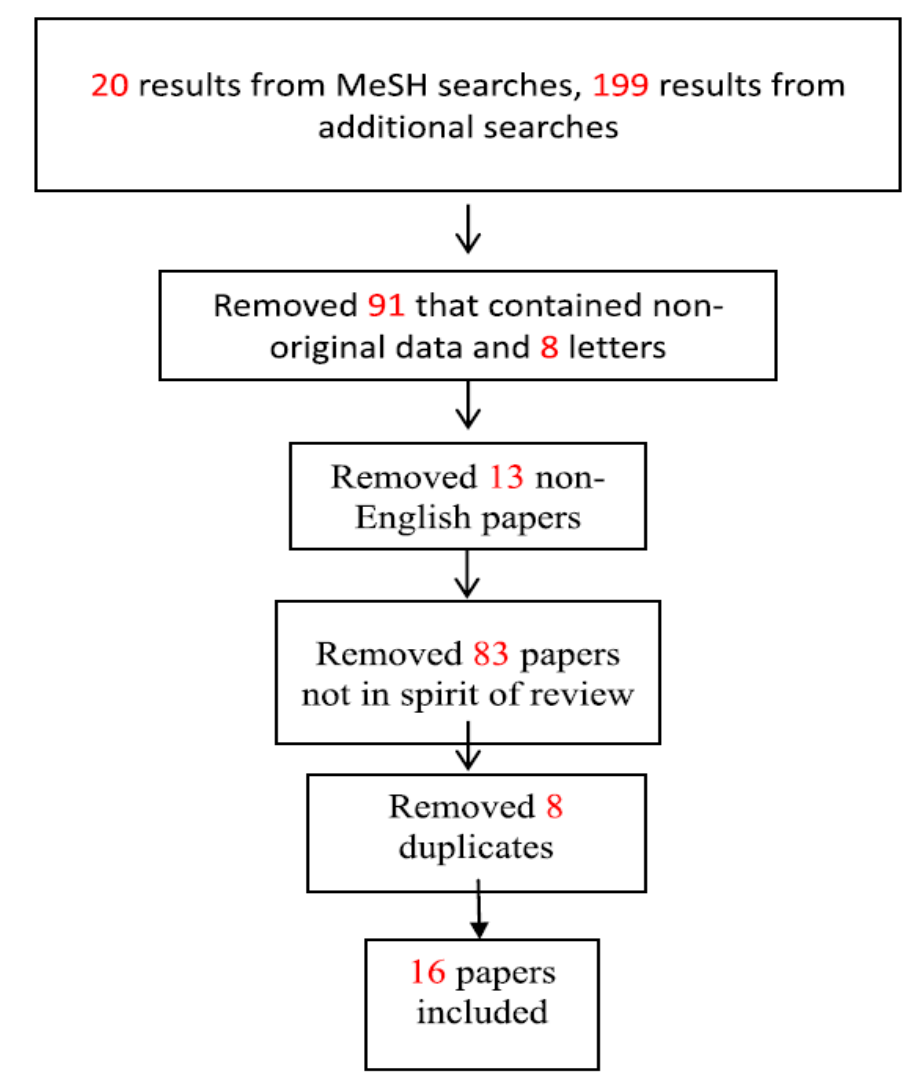

Fig. 1. Diagram of the systematic literature review of studies examining the role of epigenetics in depression and suicide.

\section{RESULTS}


The results of the review are summarized in Table 1 and discussed in more detail in the following paragraphs.

\subsection{Epigenetics and Suicide/Suicidal Ideation}

Fiori and Turecki examined postmortem brain tissue of 10 subjects who died by suicide and 10 controls (Fiori \& Turecki, 2011). Cytosine-guanine dinucleotides (CpG site) methylation at Brodmann's area (BA) 8/9 showed a negative association with SAT1 expression in the suicide group. The CPG promoter as a whole, as opposed to a part, seemed to be instrumental in determining SAT1 expression. There was increased methylation at the polymorphic CpG site created by $\mathrm{rs} 6526342$ in the group who died by suicide, indicating that methylation at this site could mediate the association between the $C$ allele and suicide.

Chromatin modifications were assessed to see how they affected SAT1 expression. H3K27me3 was studied as it seems to be unaffected by postmortem changes to the brain. However, there seemed to be no significant changes in H3K27me3 levels between the control group and the group who died by suicide, nor did the level of H3k27me3 seem to correlate with the expression of SAT1. A correlation was found between H3k27me3 and SAT1 exon methylation, which may be incidental, as it did not seem to make a difference in the expression of SAT1.

This study is valuable for its preliminary results of epigenetic changes in patients who died by suicide. However, there are several potential limitations to consider in regards to this study. First, the small sample size limits the power of the data analysis. Second, the study only included males, and therefore it is unclear if the results are generalizable to females. As the authors point out in their study, SAT1 is found on the X chromosome, and there is increased CpG methylation in females regardless of other factors. Finally, it is possible that other histone modifications which were not studied may be involved in important epigenetic changes.

Gross et al (2013) examined BA44 in postmortem brain tissue in 34 people who had died by suicide and 34 control subjects. The first thing that was examined was the level of gene expression of OAZ1, OAZ2, AMD1, and ARG2. All of these had increased levels of expression in the group of patients who died by suicide. Then, methylation level in the promoter regions of these genes was assessed. The mean methylation level was 3-8\%. OAZ1 was the only gene to show significant methylation differences between the two groups. OAZ2 showed a trend toward significance. While there was hypermethylation of specific CpGs of promoters for both OAZ1 and OAZ2, neither of these was correlated with gene expression.

Alternatively, for AMD1 there was hypomethylation in patients who died by suicide at CpG9 and CpG 16 that showed a negative correlation with gene expression. For ARG2, hypomethylation of CpGs 5-7 in patients who died by suicide was negatively correlated with gene expression.

The researchers in this study accounted for age, post-mortem interval, and if the subject was on psychiatric medications at the time of death. As these are all possible confounding variables, it is a strength of the study that these were taken into account when processing their data. Limitations, including the small sample size, might have affected the power of the study. Also, only Caucasian males were included in the study which limits the generalizability of the findings to the wider population. Finally, it is possible that, since only a limited area (500 base 
pairs) was studied, the results found were from changes further upstream than the area under investigation in this study.

Ernst et al. (2009) examined TrkBT1 methylation in patients who died of suicide as well as other causes. All subjects were French Canadian males with postmortem interval less than 48 hours. There were 28 subjects who died by suicide and 11 subjects who died of other causes. This study used extreme value analysis to find abnormalities. The researchers initially focused on BA 8/9 and found that there was reduced TrkBT1 expression in 10 of 28 subjects who died of suicide. Then, the researchers examined 8 other frontal cortical regions, and they found that there was similarly low TrkBT1 expression in these subjects in all these regions. They also examined the cerebellum but did not find a decreased expression of TrkBT1. Interestingly, the lower TrkBT1 expression was associated with 2 particular CpG sites in the TrkB promoter.

The authors of this study (Ernst et al., 2009) investigated whether PMI, pH, or age affected TrkBT1 expression, and found that they did not. A strength of this study is that the authors examined multiple brain regions in the same subjects and found that the effect held. The researcher also examined if a history of alcohol or cocaine abuse affected the TrkBT1 expression, but no effect was found. A major weakness of this study is the small number of subjects. Another concern is that the statistical method utilized, the extreme value analysis method, is a new method, and it is unclear if the results are as reliable.

In a study by Keller et al. (2011), post-mortem brain tissue from 18 subjects who died by suicide and 18 control subjects were examined, with samples collected from Wernicke's area. Contrary to studies which showed a downregulation of TrkB-T1 transcript levels in other brain areas, in this study there was no significant difference in TrkB-T1 expression and TrkB promoter methylation in Wernicke's area between the control group and the group of patients who died by suicide. The authors also extrapolated previously obtained data about BDNF promoter methylation from a previous study they had performed as well as subjects in this study, and found that the BDNF promoter was hypermethylated in patients who died of suicide; these data are in accordance with that of Kang et al. (2013) and Kim et al. (2014).

An important aspect of this study (Keller et al., 2011) is that it examined epigenetic findings in suicide in an area that has not been investigated before: Wernicke's area. Although the study had a small sample size and was preliminary in nature, it provided a base for further studies in this area (Fiori \& Turecki, 2011; Gross et al., 2013; Maussion et al., 2014). The results may not be generalizable given that only Caucasian individuals were studied.

Maussion et al. (2014) examined the epigenetics of suicide in BA 8/9 involving TrkB. Other than looking at the promoter region, this study investigated the methylation in other TrkB gene regions. A methylation study was done on 11 subjects who died by suicide and who had low TrkBT1 levels plus 13 subjects who died of other causes and who were not selected on the basis of TrkB-T1 level. Next, genotyping of the rs1624327 variant was performed on the 24 subjects that were involved in the methylation study, as well as 22 additional subjects who died by suicide and who also had low TrkB-T1 levels, plus 15 additional subjects who died of other causes and were not selected on the basis of TrkB-T1 levels. All subjects were male French Canadians and were matched according to age and postmortem interval. 
In the microarray analysis of the 11 subjects who died by suicide and had low TrkB-T1 levels and 13 subjects who died of other causes and were not selected on the basis of TrkB-T1 level, five consecutive probes were found to be hypermethylated in the subjects who died by suicide. The $1 \mathrm{~kb}$ region that encompassed these five probes was located at the TrkB 3' UTR and contained $13 \mathrm{CpGs}$. In this region, there was overall significant hypermethylation in the group who died of suicide versus the group who died of other causes. This was further broken down to indicate that the hypermethylation was clustered in CpGs6-9. From the expanded sample for rs1624327, no significant differences were found between the group that died of suicide and the group that died of other causes.

This study (Maussion et al., 2014) had some strengths, including that it built on the data of a previous study to further elucidate and differentiate the data. There were also some weaknesses, including the small sample size, especially of the microarray analysis. Additionally, the data included only males of French Canadian origin, which makes it difficult to generalize the results to a more diverse population.

Kang et al. (2013) investigated BDNF promoter methylation in patients with suicidal ideation. They examined 108 patients with a diagnosis of major depressive disorder who completed 12 weeks of outpatient evaluations. The patients involved in the study received treatment "as usual" by their psychiatrists. The patients were treated with nine antidepressants including escitalopram, paroxetine, sertraline, fluoxetine, buproprion, mirtazapine, venlafaxine, amitryptyline, and imipramine. The patients were questioned about a previous suicide attempt as well as suicidal ideation at baseline and at weeks 1, 2, 4, 8, and 12 of treatment. The Beck Scale for Suicidal Ideation was administered at baseline and at weeks 1, 2, 4, 8, and 12. Several other ratings scales were also used to quantitatively assess depressive symptoms.

There was significantly higher BDNF methylation in males, as well as higher BDNF methylation associated with lower scores on the Social and Occupational Functioning Assessment Scale (SOFAS) and World Health Organization Quality of Life Abbreviated Form (WHOQOL-BREF). No association between BDNF methylation and Hamilton Depression Rating Scale (HAMD) score was found, so the authors further separated the HAMD scores into $<20$ and $\geq 20$, but there still was no association between HAMD score and BDNF methylation.

Of the subjects, $19.4 \%$ had previous suicide attempts, $51.9 \%$ had suicidal ideation at baseline; $52.8 \%$ had suicidal ideation during treatment, and $25 \%$ had suicidal ideation at the last treatment session. Twenty-five percent of the patients had suicidal ideation during treatment but not at baseline, and $5.6 \%$ had self-harm during treatment. Previous suicide attempt, suicidal ideation during treatment, and suicidal ideation at the last treatment session were found to be significantly associated with a higher BDNF methylation level, even when adjusted for gender, SOFAS, and WHOQUOL-BREF scores. There was no significant association between antidepressant type and BDNF methylation nor between antidepressant type and suicidal outcome. Of the patients with lower BDNF methylation, there was significant improvement in Beck Scale for Suicidal Ideation scores over the 12-week treatment period. However, in patients with higher levels of BDNF methylation, there was much less improvement in Beck Scale for Suicidal Ideation scores. 
This study has several strengths. First, the investigators used several scales to objectively assess symptoms. Second, the investigators statistically controlled for antidepressant use. The major weakness of this study is the lack of a control group. Of note, in this study and in some of the others reviewed, blood samples were used. There is, however, some concern about how the results of blood samples translate with postmortem brain autopsy samples.

Kim et al. (2014) investigated the methylation of BDNF promoter in a geriatric Korean population. Community residents of two areas of Kwangju, South Korea, were asked questions about suicidal ideation at baseline and were followed up at two years. Both at baseline and at the 2-year follow-up, participants were given the Geriatric Mental State Schedule, and suicidal ideation within the past month was assessed.

Other data collected were age, sex, education level, depressive symptoms, cognitive function, and any level of disability. The Geriatric Depression Scale was used to assess level of depression, the Mini Mental State Exam to assess cognitive function, and the World Health Organization Disability Schedule II to assess level of disability.

At baseline, 732 subjects were interviewed, of which 93 endorsed suicidal ideation within the past month (Kim et al., 2014). The factors that were most commonly associated with suicidal ideation were older age, lower cognitive function, more severe depressive symptoms, and higher level of disability; hence, these factors were chosen as covariates for this study. Only subjects who did not endorse suicidal ideation at baseline were chosen to be followed (639 subjects). Of these, 57 endorsed suicidal ideation within the past month at 2-year follow-up. Of the remaining subjects, 24 were lost to follow-up, 12 had died, 10 refused to participate, 8 had changed their addresses, and 6 were too unwell to participate. There were no statistical differences between the subjects who participated in the two year follow-up and those who did not.

Suicidal ideation at baseline was significantly associated with higher methylation at BDNF promoter CpGs 5, 7, 8, and 9 (Kim et al., 2014). Suicidal ideation at 2-year follow-up was significantly associated with higher methylation at BDNF promoter CpG 9.

This study (Kim et al., 2014) has several strengths. First, it had a larger sample size than many of the other studies that have been reviewed herein, which makes the power of the data stronger. It also confirmed the findings of Kang et al. that BDNF methylation is significantly associated with suicidal ideation (Kang et al., 2013). Another strength of this study is that it had a large retention rate, and there was no statistical significance between the participants who followed up and those lost to follow-up. Additionally, there are few studies that have focused on the geriatric population in the area of epigenetics and suicidality. A weakness of the study is that it did not utilize a control group. Also, the study (Kim et al., 2014) only utilized one CpG island to assess for BDNF methylation status (similar to (Kang et al., 2013)).

Klempan et al. (2009) examined QKI gene expression and epigenetics in subjects who had died of suicide and who had major depressive disorder. The researchers assessed 16 patients who had died of suicide and 13 controls. The following brain areas were examined: BA 4, 6, 8/9, $10,11,20,21,24,29,38,44,45,46$, and 47 as well as the hippocampus, amygdala, and nucleus accumbens. The number of subjects varied by brain region, with the median for the suicide group being 10 and the median for the control group being 7. The researchers found that the QKI 
expression was significantly downregulated at most areas tested (BA4, BA8/9, BA11, BA20, BA21, BA44, BA45, BA46, and BA47). Five of the subjects had a history of alcohol abuse, and this was not found to be related to the QKI expression. The researchers had hypothesized that the difference in QKI expression was due to epigenetic changes, but found little methylation at the promoter for QKI in either group.

Previous studies had shown decreased QKI expression in schizophrenia ((Aberg et al., 2006) and (Haroutunian, Katsel, Dracheva, \& Davis, 2006)), and this study further extended that work in subjects with major depressive disorder. A limitation of this study is its small sample size. Further, it is difficult to tease out if the decrease in QKI expression was due to the history of major depressive disorder or suicidality.

Haghighi et al. (2014) examined DNA methylation in 25 subjects who died of suicide (and had major depressive disorder) as compared to 28 subjects who died of other causes (with no axis I DSM-IV diagnosis). All subjects had negative toxicological screening for psychotropic or illicit drug use in the last 3 months. The researchers tested BA47 for DNA methylation at 27,578 CpG sites. The data showed that all CpG sites had an increase in DNA methylation with age. However, in the group that died by suicide, the increase in methylation was 8 times greater.

This study has several strengths. First is the large amount of CpG sites tested. Second, the researchers excluded patients with psychotropic or illicit drug use three months premortem, which eliminated a potential confounding factor. Weaknesses include that only one brain area was tested and the small sample size. Also, increased methylation may be hard to discern if related to depression or suicide.

Nagy et al. (2015) examined epigenetic changes in astrocyte marker expression in the context of patients who have died of suicide. The researchers examined 76 subjects who died of suicide and 45 controls. Using BA10, they identified males within the lowest quartile of expression of seven genes: GFAP, ALDH1L1, SOX9, GLUL, SLC1A3, GJA1, and GJB6 (for a total of 22 subjects). Then, the control subjects were picked in order to best match the characteristics of the suicide group. Methylation analysis was done of the genome and differentially methylated regions (DMRs) were identified. The researchers chose to focus on two DMRs, GRIK2 and BEGAIN, that are important in astrocyte function. They found that GRIK2 had an increased expression in the patients who had died of suicide. For BEGAIN, there are two transcripts, but for variant 1 (which is expressed mostly in the brain), they found a decreased expression. The researchers then separated tissue into neuronal and non-neuronal fractions and found no difference for GRIK2, but found an increase in methylation in the non-neuronal fraction for BEGAIN.

This study is one of the first to use next-generation sequencing to investigate epigenetic changes, and it is a very innovative approach to investigating astrocytic epigenetic changes. One weakness is that the authors state that their data apply to subjects with depression and suicide; however, subjects with a DSM-IV diagnosis of major depressive disorder as well as with no axis I diagnosis were included in the study group. Also, a low number of patients were studied and, since the study was restricted to males, it may not be applicable to females.

\subsection{Epigenetics and Depression}


A study by Dell'Osso et al. examined the difference in BDNF promoter methylation in subjects with major depressive disorder (MDD), bipolar I disorder, bipolar II disorder, and healthy control subjects (Dell'Osso et al., 2014). The study examined 43 subjects with major depressive disorder, 61 subjects with bipolar I disorder, 50 subjects with bipolar II disorder, and 44 agematched healthy controls. The patients with psychiatric diagnoses were on stable pharmacologic treatment.

There was a similar level of BDNF DNA methylation in the MDD patients as in the bipolar II patients, both significantly more than the bipolar I patients. The authors also stratified data according to the mood state of the patients and found that BDNF DNA methylation of manic/mixed patients was comparable to healthy controls, whereas BDNF DNA methylation of depressed patients was comparable to euthymic patients. Methylation was significantly higher in depressed patients compared to manic/mixed patients. BDNF DNA methylation was found to be lowest in bipolar I disorder patients regardless of mood state as compared to patients with major depressive disorder and bipolar II disorder. Patients with bipolar II disorder in a euthymic and depressed state were found to have a higher level of BDNF DNA methylation compared to bipolar II patients in a hypomanic state.

BDNF DNA methylation was analyzed on the basis of the type of pharmacologic treatment that the patients were receiving. It was shown that, overall, lithium and valproate tended to decrease the level of methylation at the BDNF gene promoter as compared to other classes of medications (antidepressants, antipsychotics) but that this decrease was not of a statistically significant level.

There are several strengths of this study. The study identifies an epigenetic marker for depression, and further stratifies it according to manic versus depressive phases of bipolar disorder. The authors of the study also included potential confounding variables (pharmacological treatment, age, and sex) in the statistical model.

Melas et al. (2012) examined epigenetic effects associated with depression in rat models. The study examined prefrontal cortices of Flinders Sensitive Line (FSL) and Flinders Resistant Line (FRL) rats. Control rats were used as well as rats that had been treated with escitalopram from both lines. The escitalopram was administered for 3 weeks prior to the euthanasia of the rats. There were $12 \mathrm{FRL}$ rats, $14 \mathrm{FSL}, 6 \mathrm{FRL}$ treated with escitalopram, and $9 \mathrm{FSL}$ treated with escitalopram.

P11 mRNA has previously been shown to specifically interact with serotonin levels in the prefrontal cortex. The investigators assessed if there was a difference in P11 mRNA expression between the control FSL and FRL rats. There was significantly reduced P11 mRNA expression in the pre-frontal cortices of the FSL rats (which served as the model for depression). This was confirmed by Western blotting which showed decreased protein expression. The FSL rats showed hypermethylation in the P11 promoter compared to the controls. In silico analysis revealed that two CpG sites in the P11 promoter region were located within a putative binding site of the androgen receptor (AR), a DNA-binding transcription factor (TF) that inhibits corticotropinreleasing factor (CRF). Reduced AR mRNA levels have previously been reported in post-mortem brains of depressed individuals (Wang et al., 2008). However, Melas et al. did not find significant 
difference of AR mRNA levels between FSL and FRL rats, suggesting that aberrant AR mRNA levels may not underlie the depressed phenotype in this rat model.

In the escitalopram-treated rats, P11 mRNA levels were significantly higher when compared to the rats left untreated. In fact, the P11 of FSL rats treated with Escitalopram was found to be at a level that was not statistically different from the P11 level of the FRL rats.

Similarly, in the FSL rats treated with escitalopram, the level of methylation of the P11 promoter was statistically undifferentiated from the FRL group.

The mRNA levels of three DNA methyltransferases as well as four genes encoding proteins were examined to see if they are involved in the methylation changes observed. Of the methyltransferases examined (Dnmt1, Dnmt3a, Dnmt3b), Dnmt1 and Dnmt3a were significantly decreased after escitalopram treatment in the FSL group. Of the genes examined, there was no significant difference for Mbd2, Mbd4, or Gadd45b, though Gadd45b did show a trend toward significance.

As a rat study, this study occurred in a much more controlled environment than the other studies reviewed herein. On the other hand, weaknesses of the Melas et al. study may include the lack of applicability of this rat study to humans.

Davies et al. (2014) investigated human discordant twin pairs for major depressive disorder to identify epigenetic changes associated with the disorder. The study included two samples, 27 monozygotic twin (MZT) pairs from the UK and 23 MZT pairs from Australia.

The two data sets from the twin pairs were integrated through a meta-analysis (Davies et al., 2014). Genes identified in the meta-analysis as hypermethylated were evaluated in conjunction with existing literature to select for likely hypermethylated regions associated with major depressive disorder. In order to replicate these findings, blood MeDIP-seq data from 354 unrelated, age-matched females from the EpiTwin project were included, 118 suffering from major depressive disorder and 236 controls. Four DMRs (differentially methylated regions) were selected for replication. They were also compared with an independent dataset of 932 postmortem brain samples from the Edinburgh Brain Bank, which included 932 brain samples of ten different brain regions obtained from 101 unrelated individuals ( 24 male and 77 female) ages 16 to 83. The brain regions were cerebellum, frontal cortex, hippocampus, medulla, occipital cortex, putamen, substantia nigra, temporal cortex, thalamus, and intralobular white matter.

The meta-analysis of the UK and Australian data revealed 17 DMRs of genome-wide significance. Four of these were in genes related to the pathology of major depressive disorder: ZBTB20, AGTPBP1, TBC1D8, and CLSTN1. Each of these was replicated in an independent replication cohort of 354 unrelated age-matched females, and there was $28.2 \%$ increased methylation in the depressed cohort compared to the control group. In the meta-analysis, ZBTB20 was the second most differentially methylated region (of all seventeen DMRs identified).

The investigators made two additional linear mixed models with the UK data subset, as described above, in order to rule out antidepressant medication as a confounding factor for the results found, and it was found that ZBTB20 is highly methylated in subjects with major depressive disorder regardless of whether they are taking antidepressant medications. 
Additionally, examination of 932 postmortem brain samples from the Edinburgh Brain Bank from ten different brain regions found that ZBTB20 is highly expressed in the hippocampus, cerebellum, and white matter regions, and lowly expressed in the frontal, occipital, and temporal cortices.

A strength of this study (Davies et al., 2014) is that the researchers accounted for several confounding variables, including age, BMI, smoking status, and antidepressant medication. A weakness is that subjects from the two twin sets were diagnosed using different questionnaires, which could be a confounding factor. Additionally, the majority of the twin pairs studied were female, which limits the generalizability of the results for males.

Weder et al. (2014) investigated the effects of child abuse on epigenetics and depression. The study included 94 maltreated children and 96 healthy control children with no history of maltreatment or exposure to intrafamilial violence and no past psychiatric illness. The maltreated children had been removed from their parents' care. Many objective measures were used to assess diagnoses for the subjects. Of the maltreated children, 50\% met criteria for PTSD, $12 \%$ for major depressive disorder, $17 \%$ for dissociative disorder and $17 \%$ for dissociative disorder not otherwise specified (Weder et al., 2014). Of the children with depressive disorder, 88\% were comorbid for PTSD.

The maltreated children in the study had a range of 1-7 instances of substantiated abuse or neglect, with an average of 3.92\% of the children experienced more than one type of abuse or neglect: $65 \%$ of the children had experienced physical abuse, $24 \%$ sexual abuse, $83 \%$ neglect, $65 \%$ emotional abuse and $70 \%$ had witnessed domestic violence. The Mood and Feelings Questionnaire (MFQ) was used to assess the children's depression symptomatology. Twenty-six percent of maltreated children and $4 \%$ of controls met the threshold for depression (score of 27).

Three genes (ID3, TPPP, and GRIN1) had methylation values at CpG sites that were significantly related to depression scores. Greater methylation at the CpG sites (related to ID3, TPPP, and GRIN1) were associated with lower depression scores. Methylation changes at these genes appeared to be independent of maltreatment history, age, race, and gender. Additionally, methylation changes appeared to be independent of whether the children met criteria for a PTSD diagnosis.

One strength of this study is that it had two quality control measures to ensure that the data were of high standards. Additionally, the researchers employed several objective measures to diagnose their subjects. Weaknesses include that the study included only children, thus limits generalizability to adults, and the inclusion of siblings may have led to unintentional bias.

Melas et al. (2013) examined genetic and epigenetic associations of monoamine oxidase A (MAOA) gene and glucocorticoid receptor (NR3C1) gene with childhood adversities and the later development of depression. The researchers used data from the PART study, which is a longitudinal population-based mental health study of Swedish citizens. They investigated the relationship between childhood adversities, depression, and MOAO gene alleles. Childhood adversities were associated with depression in both males and females. However, females who experienced childhood adversity and had the MAOA-L allele were at higher risk than those with MAOA-H allele. There was no difference between these subtypes in men. Also, in MAOA-L 
females, exposure to one childhood adversity was associated with higher risk for depression. This association was less pronounced in males.

The researchers (Melas et al. 2013) then assessed the following childhood adversities to see if they altered methylation levels of MAOA and NR3C1 in females with depression and controls: parental death, parental divorce, financial problems, and familial constraints. They found a hypomethylation pattern of the MAOA in depressed females compared to controls and that this association was independent of the MAOA genetic variations. In addition, an association of early parental death with hypermethylation of the NR3C1 was found in females. However, this association was found only among MAOA-L but not MAOA-H, indicating that the MAOAgenotypic variations may affect the extent of NR3C1's epigenetic programming as a result of this traumatic experience.

This study (Melas et al., 2013) analyzed data from the PART study, which included 8613 randomly selected individuals. However, a subset analysis of NR3C1 included only 10 females with depression and early parental death and only 2 controls, thus making the data difficult to interpret due to its lack of power. The PART study also used saliva to collect DNA, which is unique among the studies reviewed herein, and though the researchers assert that saliva shows directly what is in blood leukocytes, this is not yet a commonly used method. The DNA methylation portion of the study included females, thus it is not generalizable to males. Another limitation in the generalization of the PART study is that it only examined the Swedish population, and the study has not been replicated in other samples.

Numata et al. (2015) examined medication-free patients with major depressive disorder in an effort to isolate biomarkers. To this end, the researchers had two parts to their study: discovery and replication. For their discovery sample, they had 20 medication-free patients with major depressive disorder ( 2 males and 18 females, only one patient who was on zolpidem) and 19 medication-free control subjects with no mental illness. For the replication study, they had 12 medication-free patients with major depressive disorder ( 3 males and 9 females, one patient was taking tandospirone) and 12 medication-free controls with no mental illness. The researchers looked for differences in DNA methylation between the two groups at 431,389 CpG sites, and differences were found at 363 sites. Of the 363 sites, all had lower methylation in the patients with major depressive disorder as compared to the controls, and notably this included some CpG sites which had previously been hypothesized to be associated with major depressive disorder, including CITED2, DGKH, GSK3B, and SGK1. The researchers then did a replication study. In this study, of the top 100 methylated CpG sites in the discovery arm, 84 were significant.

This study took a large leap forward in trying to identify epigenetic biomarkers. There were many CpG sites investigated. The authors also did both a discovery and replication step, which further validates the data. Limitations included the small number of subjects, and the much higher percentage of females. 


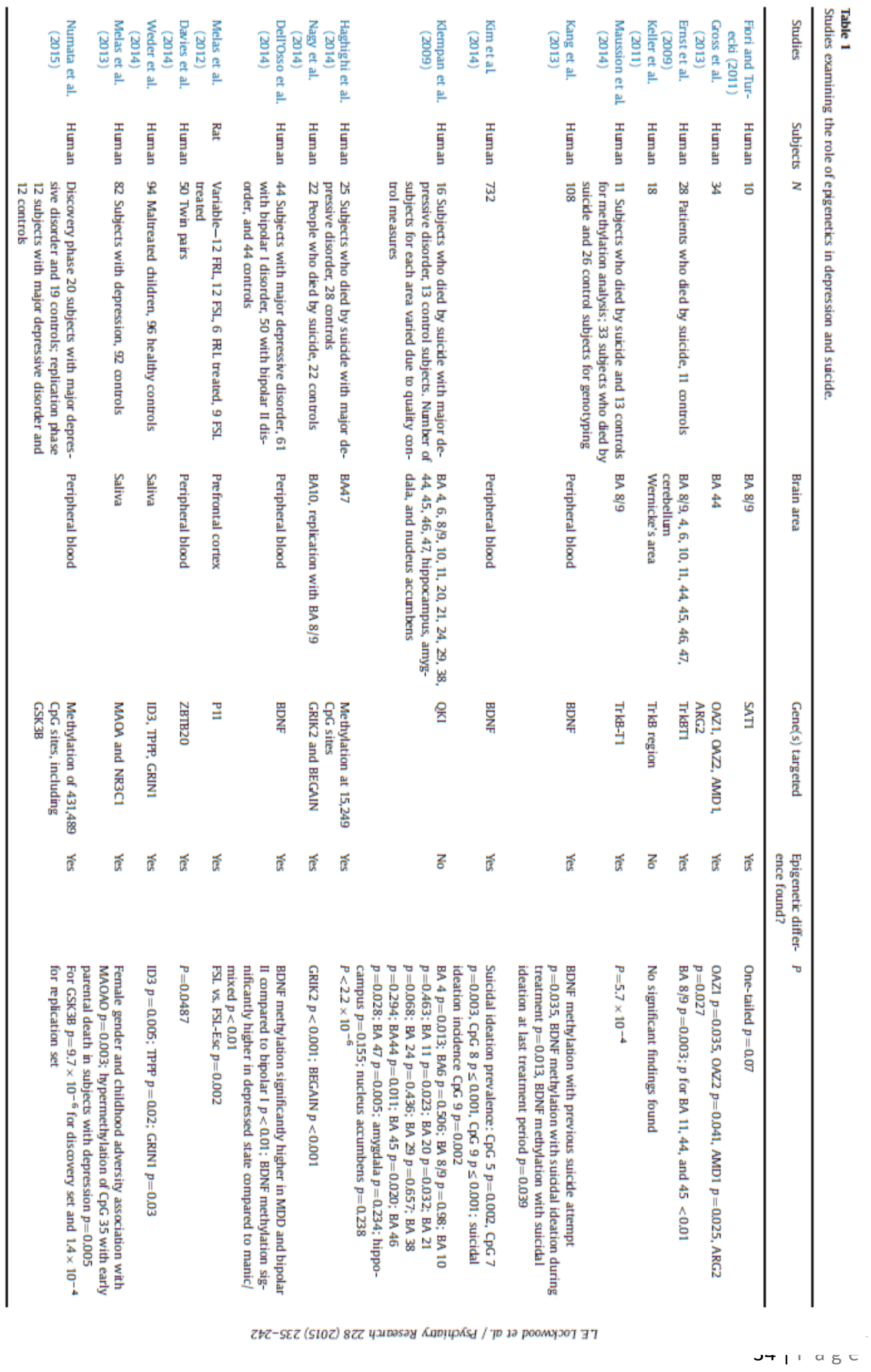




\section{DISCUSSION}

This paper reviewed the role of epigenetics on depression and suicide. Our hypothesis was that depression and suicide are associated with epigenetics alterations, which was supported by the majority of papers reviewed.

Maussion et al. (2014) examined methylation at the TrkB-T1 region. They found significant hypermethylation at this area in subjects who died of suicide in comparison to subjects who died of other causes. The TrkB region was also examined in the Keller study (Keller et al., 2011), which did not find any difference in methylation between patients who died of suicide and patients who died of alternative means.

Ernst et al. (2009) also examined the TrkB region in postmortem brain tissue of control subjects and subjects who died of suicide, both in BA8/9 as well as eight other cortical regions and the cerebellum. In the Ernst paper, a difference in TrkB methylation specific to the TrkBT1 site was found in the group of people who died by suicide. The researchers in the Ernst study did not look at Wernicke's area, which is BA22.

There are several possible reasons for the differences in these findings. First, both Ernst and Maussion examined the BA8/9 region, while Keller examined Wernicke's area. It is possible that the methylation difference found in people who died of suicide is not present in all regions of the brain. Second, all three studies had small sample sizes (Table 1). It is possible that none of these studies were powered adequately to give definitive findings. Additionally, the Ernst and Maussion studies were both performed by the same laboratory, and it is possible that some of the subjects could have been overlapping (other papers in this review performed by that laboratory include Fiori and Turecki (2011), Gross et al. (2013), Klempan et al. (2009), and Nagy et al. (2015). It is conceivable that some of the studies reviewed herein from this laboratory had overlapping samples, but it is impossible to tell this with certainty (personal correspondence with Dr. Turecki).

BDNF methylation was found to be significantly associated with suicidal ideation by Keller et al. (2011), Kang et al. (2013) and Kim et al. (2014). The Dell'Osso study (Dell'Osso et al., 2014) further examines when BDNF is hypermethylated in psychiatric illness as well as current treatments that help to decrease this methylation. Dell'Osso et al. (2014) found that BDNF methylation in patients with major depressive disorder was higher than in patients in a manic or mixed state of bipolar disorder. Further, they found that while lithium and valproate decreased the level of BDNF methylation, the decrease was not statistically significant.

This review paper has some weaknesses, in that the studies reviewed were all preliminary in nature and had small sample sizes. Nonetheless, most studies were in agreement concerning the association between depression and suicide and epigenetic alterations. Another possible limitation of our study is publication bias. It is possible that researchers who did not find a role for epigenetics in depression and suicide may not have published their studies. Another limitation is the possible overlap of samples in six of the papers reviewed, which could account for some of the results seen.

However, the agreement among most of the reviewed studies is highly suggestive that epigenetics may play an important role in depression and suicide. This can provide an important 
biomarker in predicting disease state and disease stratification that might help move the field forward towards precision medicine. Moreover, as epigenetic modifications are functional modifications, rather than structure changes in the genome, epigenetic modifications may offer an easier and more effective mode for future therapeutic methods. Thus, further studies to better characterize epigenetic modifications are promising. Epigenetics has a promising role in the future for both new therapeutic avenues and as a possible psychiatric biomarker, but more research in this field is needed. 
Contributions (added for the purpose of this thesis): Nagy Youssef conceptualized the idea of the manuscript, developed the inclusion criteria and search strategy, and mentored Laura Lockwood, his resident physician at the time of this systematic review. They searched the literature, and both contributed equally to the text and edited the paper of the initial draft. Shaoyong Su provided input on the later draft in conceptualization and editing, and all coauthors approved the final version of the manuscript. Also, of note, none of the chapters used in this thesis, to my knowledge, have been used for any other PhD thesis. 


\section{REFERENCES}

Aberg, K., Saetre, P., Lindholm, E., Ekholm, B., Pettersson, U., Adolfsson, R., \& Jazin, E. (2006). Human QKI, a new candidate gene for schizophrenia involved in myelination. Am J Med Genet B Neuropsychiatr Genet, 141b(1), 84-90. doi:10.1002/ajmg.b.30243

Davies, M. N., Krause, L., Bell, J. T., Gao, F., Ward, K. J., Wu, H., . . Wang, J. (2014). Hypermethylation in the ZBTB20 gene is associated with major depressive disorder. Genome Biol, 15(4), R56. doi:10.1186/gb-2014-15-4-r56

Dell'Osso, B., D'Addario, C., Carlotta Palazzo, M., Benatti, B., Camuri, G., Galimberti, D., . . . Altamura, A. C. (2014). Epigenetic modulation of BDNF gene: differences in DNA methylation between unipolar and bipolar patients. J Affect Disord, 166, 330-333. doi:10.1016/j.jad.2014.05.020

Ernst, C., Deleva, V., Deng, X., Sequeira, A., Pomarenski, A., Klempan, T., . . Turecki, G. (2009). Alternative splicing, methylation state, and expression profile of tropomyosin-related kinase B in the frontal cortex of suicide completers. Arch Gen Psychiatry, 66(1), 22-32. doi:10.1001/archpsyc.66.1.22

Fiori, L. M., \& Turecki, G. (2011). Epigenetic regulation of spermidine/spermine N1acetyltransferase (SAT1) in suicide. J Psychiatr Res, 45(9), 1229-1235. doi:10.1016/j.jpsychires.2011.03.015

Goldberg, A. D., Allis, C. D., \& Bernstein, E. (2007). Epigenetics: a landscape takes shape. Cell, 128(4), 635-638. doi:10.1016/j.cell.2007.02.006

Gross, J. A., Fiori, L. M., Labonte, B., Lopez, J. P., \& Turecki, G. (2013). Effects of promoter methylation on increased expression of polyamine biosynthetic genes in suicide. $J$ Psychiatr Res, 47(4), 513-519. doi:10.1016/j.jpsychires.2012.11.016

Haghighi, F., Xin, Y., Chanrion, B., O'Donnell, A. H., Ge, Y., Dwork, A. J., . . Mann, J. J. (2014). Increased DNA methylation in the suicide brain. Dialogues Clin Neurosci, 16(3), 430-438.

Haroutunian, V., Katsel, P., Dracheva, S., \& Davis, K. L. (2006). The human homolog of the QKI gene affected in the severe dysmyelination "quaking" mouse phenotype: downregulated in multiple brain regions in schizophrenia. Am J Psychiatry, 163(10), 1834-1837. doi:10.1176/ajp.2006.163.10.1834

Kang, H. J., Kim, J. M., Lee, J. Y., Kim, S. Y., Bae, K. Y., Kim, S. W., . . Yoon, J. S. (2013). BDNF promoter methylation and suicidal behavior in depressive patients. J Affect Disord, 151(2), 679-685. doi:10.1016/j.jad.2013.08.001

Keller, S., Sarchiapone, M., Zarrilli, F., Tomaiuolo, R., Carli, V., Angrisano, T., . . Chiariotti, L. (2011). TrkB gene expression and DNA methylation state in Wernicke area does not associate with suicidal behavior. J Affect Disord, 135(1-3), 400-404.

doi:10.1016/j.jad.2011.07.003 
Kim, J. M., Kang, H. J., Bae, K. Y., Kim, S. W., Shin, I. S., Kim, H. R., . . Yoon, J. S. (2014). Association of BDNF promoter methylation and genotype with suicidal ideation in elderly Koreans. Am J Geriatr Psychiatry, 22(10), 989-996. doi:10.1016/j.jagp.2014.02.011

Klempan, T. A., Ernst, C., Deleva, V., Labonte, B., \& Turecki, G. (2009). Characterization of QKI gene expression, genetics, and epigenetics in suicide victims with major depressive disorder. Biol Psychiatry, 66(9), 824-831. doi:10.1016/j.biopsych.2009.05.010

Maussion, G., Yang, J., Suderman, M., Diallo, A., Nagy, C., Arnovitz, M., . . Turecki, G. (2014). Functional DNA methylation in a transcript specific 3'UTR region of TrkB associates with suicide. Epigenetics, 9(8), 1061-1070. doi:10.4161/epi.29068

McGowan, P. O., \& Szyf, M. (2010). The epigenetics of social adversity in early life: implications for mental health outcomes. Neurobiol Dis, 39(1), 66-72. doi:10.1016/j.nbd.2009.12.026

Melas, P. A., Rogdaki, M., Lennartsson, A., Bjork, K., Qi, H., Witasp, A., . . L Lavebratt, C. (2012). Antidepressant treatment is associated with epigenetic alterations in the promoter of P11 in a genetic model of depression. Int J Neuropsychopharmacol, 15(5), 669-679. doi:10.1017/s1461145711000940

Melas, P. A., Wei, Y., Wong, C. C., Sjoholm, L. K., Aberg, E., Mill, J., . . . Lavebratt, C. (2013). Genetic and epigenetic associations of MAOA and NR3C1 with depression and childhood adversities. Int J Neuropsychopharmacol, 16(7), 1513-1528. doi:10.1017/s1461145713000102

Nagy, C., Suderman, M., Yang, J., Szyf, M., Mechawar, N., Ernst, C., \& Turecki, G. (2015). Astrocytic abnormalities and global DNA methylation patterns in depression and suicide. Mol Psychiatry, 20(3), 320-328. doi:10.1038/mp.2014.21

Numata, S., Ishii, K., Tajima, A., Iga, J., Kinoshita, M., Watanabe, S., . . Ohmori, T. (2015). Blood diagnostic biomarkers for major depressive disorder using multiplex DNA methylation profiles: discovery and validation. Epigenetics, 10(2), 135-141. doi:10.1080/15592294.2014.1003743

Wankerl, M., Miller, R., Kirschbaum, C., Hennig, J., Stalder, T., \& Alexander, N. (2014). Effects of genetic and early environmental risk factors for depression on serotonin transporter expression and methylation profiles. Transl Psychiatry, 4, e402. doi:10.1038/tp.2014.37

Weder, N., Zhang, H., Jensen, K., Yang, B. Z., Simen, A., Jackowski, A., . . Kaufman, J. (2014). Child abuse, depression, and methylation in genes involved with stress, neural plasticity, and brain circuitry. J Am Acad Child Adolesc Psychiatry, 53(4), 417-424.e415. doi:10.1016/j.jaac.2013.12.025

Yan, J. (5 Mar 2010). Epigenetics links nature and nurture. Psychiatric News, 45(5), 12-12. 



\title{
Chapter 3 The Effects of Trauma, With or Without PTSD, on the Transgenerational DNA Methylation Alterations in Human Offspring
}

\author{
Nagy A. Youssef ${ }^{1,2}{ }^{*}$, Laura Lockwood ${ }^{3}$, Shaoyong Su ${ }^{4}$, Guang Hao ${ }^{4}$ and Bart P. F. Rutten ${ }^{5}$ \\ 1 Department of Psychiatry \& Health Behavior, Medical College of Georgia at Augusta \\ University, 30912 Augusta, GA, USA \\ 2 Academic Affairs, Medical College of Georgia, Augusta University, 30912 Augusta, GA, USA \\ 3 Department of Psychiatry and Neuropsychology, University of Alabama at Birmingham, \\ 35233 Birmingham, AL, USA; llockwood@uabmc.edu \\ 4 Department of Population Health Sciences, Medical College of Georgia, Augusta University, \\ 30912 Augusta, GA, USA; SSU@augusta.edu (S.S.); GHAO@augusta.edu (G.H.) \\ 5 Department of Psychiatry and Neuropsychology, Maastricht University Medical Centre, \\ School for Mental Health and Neuroscience, 6202 AZ Maastricht, The Netherlands; \\ b.rutten@maastrichtuniversity.nl
}

* Correspondence: nyoussef@augusta.edu; Tel: +1-706-721-6963; Fax: +1-706-434-3200

Published as: Youssef NA, Lockwood L, Su S, Hao G, Rutten BPF. The Effects of Trauma, with or without PTSD, on the Transgenerational DNA Methylation Alterations in Human Offsprings. Brain Sci. 2018 May 8;8(5). pii: E83. doi: 10.3390/brainsci8050083. Review. PubMed PMID:

29738444; PubMed Central PMCID: PMC5977074.

https://www.mdpi.com/2076-3425/8/5/83 


\begin{abstract}
Exposure to psychological trauma is a strong risk factor for several debilitating disorders including post-traumatic stress disorder (PTSD) and depression. Besides the impact on mental well-being and behavior in the exposed individuals, it has been suggested that psychological trauma can affect the biology of the individuals, and even have biological and behavioral consequences on the offspring of exposed individuals. While knowledge of possible epigenetic underpinnings of the association between exposure to trauma and risk of PTSD have been discussed in several reviews, it remains to be established whether or not trauma-induced epigenetic modifications can be passed from traumatized individuals to subsequent generations of offspring. The aim of this paper is to review the emerging literature on the evidence of transgenerational inheritance due to trauma exposure on the epigenetic mechanism of DNA methylation in humans. Our review found an accumulating amount of evidence that the enduring effect of trauma exposure can be passed down to offspring transgenerationally (via the epigenetic inheritance mechanism of DNA methylation alterations), which has the capacity to change the expression of genes and the metabolome. This manuscript summarizes and critically reviews the relevant original human studies in this area. Thus, it provides an overview of where we stand, and a clearer vision of where we should go in terms of future research directions.
\end{abstract}

\title{
Keywords
}

psychological trauma; post-traumatic stress disorder; epigenomics; DNA methylation; prevention; treatment; transgenerational effect; transgenerational inheritance 


\section{INTRODUCTION}

Post-traumatic stress disorder (PTSD) is characterized by four symptom clusters as defined by the Diagnostic and Statistical Manual, Fifth Edition (DSM-5) (American Psychiatric Association, 2013): re-experiencing, avoidance of stimuli associated with the trauma, negative cognitions and affect associated with the trauma, and hyperarousal symptoms and signs. Fifty to $85 \%$ of Americans experience at least one traumatic event during their lifetimes, but only $7.8 \%$ go on to develop PTSD (Kessler \& Wang, 2008). Thus, the question that frequently arises is: why do some people develop PTSD after experiencing trauma, while others do not? Possible explanations could be: an underlying genetic or epigenetic risk in those who are more prone to develop PTSD and/or a protective (epi)genetic makeup, or some form of high psychological resiliency in those who do not develop PTSD or who quickly recover from PTSD, i.e., resilient individuals.

PTSD has long been established to be due to exposure to trauma, and it has been assumed that only environmental factors could contribute to the development of PTSD. On the other hand, heritability of PTSD has been estimated to be between $30 \%$ and $70 \%$ in twin studies (Sartor et al., 2012; Sartor et al., 2011; Stein, Jang, Taylor, Vernon, \& Livesley, 2002; True et al., 1993; Xian et al., 2000). Genetic studies of both candidate genes and genome-wide association studies (GWAS) have provided many interesting and promising findings, yet (so far) no robust genetic variants for PTSD have been identified (Logue et al., 2015). This risk is unlikely to be fully explained by only structural genetics (Ratanatharathorn et al., 2017).

Thus, the field of epigenetics could offer insights into differential susceptibility of risk to develop psychopathology. According to Goldberg et al. (Goldberg, Allis, \& Bernstein, 2007), epigenetics is the study of functionally stable and ideally heritable changes in gene expression or cellular phenotype that occurs without changes in base pairing. In other words, epigenetics involves functional changes to the gene without sequence changes. The best-studied epigenetic mechanism so far is via DNA methylation. We define DNA methylation as the attachment of methyl groups to the DNA molecule. When methyl groups are attached to the promoter, they typically act to repress gene transcription.

DNA methylation changes in genes have been associated with several psychiatric dysfunctions. For instance, we previously found an association between DNA methylation and both depression and suicide (Lockwood, Su, \& Youssef, 2015). Hypermethylation of BDNF promoter or TrkB were especially involved in suicide (Lockwood et al., 2015). Another equally interesting area of research is the epigenetic changes associated with PTSD.

Only a few reviews have thoroughly examined this area of the literature. For instance, a review by Ramo-Fernandez et al. (Ramo-Fernandez, Schneider, Wilker, \& Kolassa, 2015) focused primarily on the epigenetic changes associated with war trauma and childhood maltreatment. Also, a helpful review by Vinkers et al. discussed the effect of trauma on DNA methylation changes in humans and categorized the studies reviewed according to the timing of trauma exposure throughout the life span (Vinkers et al., 2015). The authors pointed out "there are significant drawbacks in the existing human literature" including "lack of longitudinal studies, methodological 
heterogeneity, selection of tissue type, and the influence of developmental stage and trauma type on methylation outcomes" (Vinkers et al., 2015). A recent longitudinal study by Rutten et al. examined the genome-wide blood DNA methylation profile changes as associated with the development of PTSD symptoms over time in two military cohorts (discovery, $N=93$, and replication data sets, $N=98$ ) (Rutten et al., 2018). The researchers found that development of PTSD symptoms over time in combat soldiers was significantly associated with DNA methylation changes (Rutten et al., 2018). The researchers suggested that the DNA methylation mediated the relation between combat trauma and PTSD symptoms longitudinally (Rutten et al., 2018).

Another equally important idea that has yet to be explored is that epigenetic modifications may mediate the impact of traumatization of parents to be passed to their offspring. Evidence from epigenetic cell and animal studies has spurred this understanding. In addition, initial recent studies in humans support these notions. The best studied of all the epigenetic mechanisms is the DNA methylation mechanism.

Since trauma and PTSD models in animals may not be well characterized (and it is not very clear how much of the animal knowledge could translate to humans), we find that in this case human studies could provide valuable insight in this area. Despite the limited literature to date in humans, a review of the transgenerational heritability as it pertains to trauma will provide both clinicians and researchers with an overview on where we stand and a clearer vision of where we should go in terms of future research directions. Transgenerational epigenetic transmission is defined as the transmission of genomic information (in this paper, DNA methylation) from one generation to the next without changing the main structure of DNA (i.e., nucleotide sequence).

Also, it should be noted that there are 2 subtypes of studies reviewed below, which involve transgenerational effects of DNA methylation changes. In the first subtype, the samples of mothers were pregnant during the time of trauma. In the second subtype, the mothers were not pregnant, and the trauma occurred before pregnancy. It seems for the latter, methylation changes to the mothers' DNA is a necessity for the transmission to occur transgenerationally. On the other hand, when trauma occurs during pregnancy, through methylation changes, the changes in the mothers are (in theory) not a necessity for the changes in the offspring to occur. While the mechanism and intensity of transgenerational genomic transmission (among other factors) may differ between these types of studies, the field has yet to clarify these differences in future research.

It is still unclear whether trauma-induced epigenetic modifications can be passed from traumatized individuals to subsequent generations of offspring. Thus, the key question of this paper is whether trauma-induced DNA methylation modifications can be passed from traumatized individuals to subsequent generations of offspring. The aim of this paper is thus to review the emerging literature on the transgenerational effects of trauma on DNA methylation in humans and to provide insights from the current literature (PubMed was reviewed from inception until March 2018), then end with recommendations for future research directions.

\section{STUDIES ON TRANSGENERATIONAL EFFECT OF TRAUMA AND PTSD}


Perroud et al. (Perroud et al., 2014) examined the impact of the Tutsi genocide on the children of the women who were pregnant while genocide was ongoing in Rwanda (which took place in 1995). In 2011, more than 20\% of the Rwandan population met criteria for PTSD (Munyandamutsa, Mahoro Nkubamugisha, Gex-Fabry, \& Eytan, 2012). The authors investigated if the risk for PTSD had been associated with epigenetic modifications in the children of women who were pregnant at the time of the genocide (Perroud et al., 2014). Twenty-five widows and their children were included in the study, as well as 25 Rwandan control women who were pregnant at the time, but who were living abroad. Peripheral blood leukocytes were obtained and methylation levels of the promoter regions of the glucocorticoid receptor $N R 3 C 1$ and the mineralocorticoid receptor $N R 3 C 2$ were examined. Cortisol levels, mineralocorticoid, and other glucocorticoid levels were also measured. As expected, both the mothers exposed to genocide and their children had significantly higher levels of PTSD and depression than the control group. They also showed higher methylation levels at exon $1 \mathrm{~F}$ promoter of NR3C1, at CpG3-CpG9. Methylation at NR3C2 was not statistically significantly different between the two groups. Mothers and children exposed to trauma had a lower cortisol level than non-exposed mothers and their children (lower cortisol levels were found to be related to PTSD (Yehuda \& Bierer, 2008)). There was a negative correlation between NR3C1 methylation and glucocorticoid levels in plasma, but no correlation was found between mineralocorticoid level and NR3C2 methylation level (Perroud et al., 2014). This interesting study by Perroud et al. highlighted the methylation changes in exposed individuals as well as in their children (Perroud et al., 2014) and suggested that traumainduced methylation changes in humans can be transmitted from parents to children. Limitations of the study include small sample size and possible confounding of the results. Confounding might occur due to the following reasons in this study: (1) since the 2 groups lived in different countries, unknown confounding variables that might have influenced the comparison with the exposed group might have affected the results; (2) the authors did not mention matching the exposed group versus the control group for possible confounders; (3) other confounding factors, such as health of participants at the time of their pregnancy, parenting style, alcohol consumption, tobacco use, and dietary factors could have played a role.

Yehuda and Daskalakis et al. (Yehuda et al., 2016) examined transgenerational methylation changes of Holocaust survivors on FKBP5, a moderator of glucocorticoid activity. The researchers examined 32 Holocaust survivors and their 22 offspring as well as 8 control subjects and their 9 offspring. Blood samples were obtained for quantification of FKBP5 methylation and cortisol levels. There were significantly higher FKBP5 intron 7 methylation levels in Holocaust survivors, but significantly lower FKBP5 intron 7 methylation levels in their offspring. The authors suggested that this opposite effect seen on FKBP5 intron 7 methylation levels might be attributable to biological accommodation in the offspring. More research is needed to replicate these findings in a larger cohort. Limitations of the study include its limited sample size as well as the presence of other factors which are impossible to control for in the population, such as the extreme starvation conditions of the Holocaust survivors, which could have also contributed to the effect seen. Despite the limitations of this study, it is quite a unique and informative study. If further replicated in a larger study, these findings could change the way our field conceptualizes trauma and PTSD. 


\section{TRANSGENERATIONAL EFFECTS OF TRAUMA AND STRESS AND PHYSICAL HEALTH}

Although the main focus of the review is on the psychological and psychiatric effect of trauma transgenerationally via methylation changes, another area that could be impacted by trauma and stress (and supported by emerging and preliminary evidence) is that the transgenerational effects may not only affect psychological health but also physical health (Hao, Youssef, Davis, \& Su, 2018). These physical effects are briefly demonstrated in the following studies.

A study by Mulligan et al. (2012) tested if prenatal maternal stress in the Congo population resulted in glucocorticoid receptor $N R 3 C 1$ methylation changes in the offspring (which may lead to increasing risk of adult-onset disease) (Mulligan, D'Errico, Stees, \& Hughes, 2012). The researchers found that, in 25 mother-newborn dyads, there was a significant correlation between prenatal maternal stress and increased methylation levels in the promoter of the glucocorticoid receptor gene (NR3C1) found in the newborns. Maternal stress was also correlated with low birth weight. They suggested that increased methylation may "constrain plasticity in subsequent gene expression and restrict the range of stress adaptation responses possible in affected individuals," thus increasing the risk for chronic diseases later in life.

For birth weight, they found that among the stressors examined, war stress has the strongest correlation, as well as the largest effect size, accounting for $35 \%$ of the variance in birth weight (Pearson's correlation $=-0.62, p=0.0009$ ). Within all war stress variables, rape accounted for $31 \%$ of birth weight variance "and eclipses the effect of other war stressors".

This increased methylation of the NR3C1 gene could increase stress reactivity and could have a long-lasting effect on vulnerability to stress, trauma, and chronic disease development.

A study by Radtke et al. (2011) examined the methylation of $10 \mathrm{CpG}$ sites in the promoter region of the $N R 3 C 1$ gene in mothers who suffered pre-pregnancy or during pregnancy exposure to intimate partner violence (IPV) and their children at the age of 10-19 (Radtke et al., 2011). According to the researchers, this was the first study to examine the effect of the trauma (IPV) over the long term (i.e., at offspring age of 10-19 years). The IPV was assessed retrospectively using the composite abuse scale (CAS) (Hegarty, Sheehan, \& Schonfeld, 1999). The study showed methylation of the NR3C1 promotor gene of the children is influenced by their mother's experience of IPV during pregnancy. There was no association between the mother's NR3C1 methylation and IPV. The authors concluded that as "these sustained epigenetic modifications are established in utero, we consider this to be a plausible mechanism by which prenatal stress may program adult psychosocial function".

However, we would like to note that the relationship may or may not be causative, as this study only showed an association, and thus causation cannot be inferred based only on this study. Other limitations of this study include: the limited sample size of 25 and the retrospective recall of IPV with the possibility (though unlikely) of inaccurate recall. However, if replicated, this finding would be important, as this is the first study to show the long-term persistence (over many years) of trauma-associated methylation of the regulator gene of the HPA-axis in the offspring. Moreover, a general caution is that, although the reviewed studies in this section did not actually address the long-term physical effects directly, they are assumed based on the 
results. For instance, the Mulligan et al. (2012) study only examined birth weight, which is not a long-term effect (Mulligan et al., 2012). However, low birth weight has certainly been linked to chronic health effects later in life (as has been shown in several other studies) (Hack, Klein, \& Taylor, 1995; Reyes \& Manalich, 2005).

\section{DISCUSSION}

The limited available literature in humans suggests that children of parents who have suffered from extreme trauma have methylation modifications associated with trauma and PTSD. This was also observed in studies done in different populations, as in the case of Yehuda and Daskalakis et al. (Yehuda et al., 2016) and Perroud et al. (Perroud et al., 2014). This may support the combined influence, not only of environmental trauma, but also of the biological component of PTSD risk. Moreover, this PTSD risk can be passed from generation to generation. Of interest, the environmental transgenerational effects that lead to change in DNA methylation in offspring has also been demonstrated in several animal models. For instance, dietary supplements during pregnancy was associated with increased methylation (of the Agouti coat color gene) and causes permanent change in coat color (Dolinoy, Weidman, Waterland, \& Jirtle, 2006; Waterland \& Jirtle, 2003), suggesting that prenatal and natal environmental interventions could induce epigenetic alterations with robust impact on stress-related disorders. These environmental interventions could range from trauma and stress to dietary and pharmacological interventions (Lockwood \& Youssef, 2017).

We want to point out, however, that in the Yehuda and Daskalakis et al. (Yehuda et al., 2016) study, the DNA methylation seen in the offspring was noted to be opposite from that seen in their parents. Although this is a perplexing finding, one possibility is that it may be a compensatory mechanism, as has been seen in Holocaust survivors who suffered extreme starvation (which is another environmental factor that can affect the epigenome) and whose offspring were more prone to metabolic syndrome (Flory, Bierer, \& Yehuda, 2011).

However, in line with the well-established notion that glucocorticoids are stress hormones, many of the studies reviewed found that the glucocorticoid receptor (NR3C1) gene is associated with methylation changes. For instance, maternal exposure to intimate partner violence during pregnancy was associated with increased NR3C1 DNA methylation in teenage children (Radtke et al., 2011). Maternal exposure to war violence or rape during pregnancy was associated with increased methylation in the NR3C1 promoter region in newborns (Mulligan et al., 2012; Rodney \& Mulligan, 2014).

Some weaknesses of this review and the current literature include the limited number of studies, most of which had small sample size. It is also unknown if there was publication bias related to studies on this topic, where studies with negative results were not published. Also, there may have been other confounding factors which could have led to epigenetic changes that were not accounted for by the studies. Moreover, several of the studies represent cross-sectional studies, where causation cannot be inferred directly from these studies.

It should be noted that these findings are still preliminary and should be taken with caution. Thus, the findings need to be replicated in larger studies with control groups, which will 
also increase our understanding, further specify, and solidify these findings. Future studies should also (as some of the studies reviewed did) clarify the timing of the trauma: whether it occurred prior to pregnancy or during pregnancy. In addition, further differences based on the trimester of pregnancy may be important to know. Also, the chronicity versus acuity of the transgenerational trauma needs to be considered and studied. Future research on this topic may lead to identification of biomarkers of trauma and PTSD risk and to greater advances into the prevention and treatment of PTSD. In addition, future studies should also follow the research recommendations of the National Advisory Mental Health Council Workgroup on Genomics (NAMHC), including using strict standards of statistical rigor for disease association, moving away from the candidate gene approaches, which have not been very fruitful, and moving towards well-powered genetic and epigenetic association studies (Gordon, 29 Mar 2018; National Advisory Mental Health Council Workgroup on Genomics, 2018). Expanding the genetic association studies beyond the DSM nosology, for instance, studying the effect of trauma per se on and the methylation changes that associate with the development of psychopathology in general (and not necessarily PTSD alone), would be helpful (since heritability is shared across psychiatric disorders). Replicating the finding in diverse populations and identifying the differences that may be unique to a particular population would be helpful. This will require data sharing between many centers to generate an adequate sample size as well as strong, meaningful significances in genome-wide studies as indicated also by the NAMHC (National Advisory Mental Health Council Workgroup on Genomics, 2018).

If the findings in the reviewed studies are confirmed definitively and further specified, it can both inform clinical research, as well as prove clinically beneficial in predicting risk to PTSD development as well as treatment planning.

\section{CONCLUSIONS}

Despite the limitations of the current literature, there seems to be accumulating evidence to suggest the transgenerational transmission of DNA methylation changes from parents to children. This area merits further replication of the presented findings. In addition, as some of the studies reviewed did, future studies should also clarify the timing of the trauma (of whether it occurred prior to pregnancy or during pregnancy), and if there are further differences based on the trimester of pregnancy. Also, the chronicity versus acuity of the transgenerational trauma needs to be considered and studied. Future research of this topic may lead to identification of biomarkers of trauma and PTSD risk and to greater advances in the prevention and treatment of PTSD. 
Acknowledgments: We thank and acknowledge the help of Mark Yassa for editorial assistance.

Author Contributions: Nagy Youssef conceptualized the study and Laura Lockwood did the initial search and extracted the relevant studies. Nagy Youssef also searched and verified the study extraction. They both extracted the data independently and discussed and reconciled any inconsistency and wrote several iterations of the manuscript. Shaoyong Su, Guang Hao and Bart P. F. Rutten edited and contributed intellectually to parts of the manuscript. All the authors approved the final version of the paper.

Conflicts of Interest: Youssef receives support from the Office of Academic Affairs, Medical College of Georgia at Augusta University, and a research grant from Augusta Biomedical Research Corporation. Lockwood, Su, Hao, and Rutten declare no relevant perceived or actual conflict of interest relevant to the content of this manuscript. 


\section{REFERENCES}

American Psychiatric Association. (2013). Diagnostic and statistical manual of mental disorders: DSM-5. Arlington, VA: American Psychiatric Association.

Dolinoy, D. C., Weidman, J. R., Waterland, R. A., \& Jirtle, R. L. (2006). Maternal genistein alters coat color and protects Avy mouse offspring from obesity by modifying the fetal epigenome. Environ Health Perspect, 114(4), 567-572. doi:10.1289/ehp.8700

Flory, J. D., Bierer, L. M., \& Yehuda, R. (2011). Maternal exposure to the holocaust and health complaints in offspring. Dis Markers, 30(2-3), 133-139. doi:10.3233/DMA-2011-0748

Goldberg, A. D., Allis, C. D., \& Bernstein, E. (2007). Epigenetics: a landscape takes shape. Cell, 128(4), 635-638. doi:10.1016/j.cell.2007.02.006

Gordon, J. A. (29 Mar 2018). Towards a Genomic Psychiatry: Recommendations of the Genomics Workgroup of the NAMHC. Retrieved from https://www.nimh.nih.gov/about/director/messages/2018/towards-a-genomicpsychiatry-recommendations-of-the-genomics-workgroup-of-the-namhc.shtml

Hack, M., Klein, N. K., \& Taylor, H. G. (1995). Long-term developmental outcomes of low birth weight infants. Future Child, 5(1), 176-196. doi:10.2307/1602514

Hao, G., Youssef, N. A., Davis, C. L., \& Su, S. (2018). The role of DNA methylation in the association between childhood adversity and cardiometabolic disease. Int J Cardiol, 255, 168-174. doi:10.1016/j.ijcard.2017.12.063

Hegarty, K., Sheehan, M., \& Schonfeld, C. (1999). A multidimensional definition of partner abuse: development and preliminary validation of the composite abuse scale. J Fam Violence, 14(4), 399-415. doi:10.1023/A:1022834215681

Kessler, R. C., \& Wang, P. S. (2008). The descriptive epidemiology of commonly occurring mental disorders in the United States. Annu Rev Public Health, 29, 115-129. doi:10.1146/annurev.publhealth.29.020907.090847

Lockwood, L. E., Su, S., \& Youssef, N. A. (2015). The role of epigenetics in depression and suicide: a platform for gene-environment interactions. Psychiatry Res, 228(3), 235-242. doi:10.1016/j.psychres.2015.05.071

Lockwood, L. E., \& Youssef, N. A. (2017). Systematic review of epigenetic effects of pharmacological agents for bipolar disorders. Brain Sci, 7(11), 154. doi:10.3390/brainsci7110154

Logue, M. W., Amstadter, A. B., Baker, D. G., Duncan, L., Koenen, K. C., Liberzon, I., . . Ressler, K. J. (2015). The Psychiatric Genomics Consortium Posttraumatic Stress Disorder Workgroup: posttraumatic stress disorder enters the age of large-scale genomic collaboration. Neuropsychopharmacology, 40(10), 2287-2297. doi:10.1038/npp.2015.118 
Mulligan, C. J., D'Errico, N. C., Stees, J., \& Hughes, D. A. (2012). Methylation changes at NR3C1 in newborns associate with maternal prenatal stress exposure and newborn birth weight. Epigenetics, 7(8), 853-857. doi:10.4161/epi.21180

Munyandamutsa, N., Mahoro Nkubamugisha, P., Gex-Fabry, M., \& Eytan, A. (2012). Mental and physical health in Rwanda 14 years after the genocide. Soc Psychiatry Psychiatr Epidemiol, 47(11), 1753-1761. doi:10.1007/s00127-012-0494-9

National Advisory Mental Health Council Workgroup on Genomics. (2018). Opportunities and Challenges of Psychiatric Genetics. Retrieved from Bethesda, MD; National Advisory Mental Health Council (NAMHC), National Institutes of Health (NIH): https://www.nimh.nih.gov/about/advisory-boards-and-groups/namhc/reports/reportof-the-national-advisory-mental-health-council-workgroup-on-genomics.shtml

Perroud, N., Rutembesa, E., Paoloni-Giacobino, A., Mutabaruka, J., Mutesa, L., Stenz, L., . . . Karege, F. (2014). The Tutsi genocide and transgenerational transmission of maternal stress: epigenetics and biology of the HPA axis. World J Biol Psychiatry, 15(4), 334-345. doi:10.3109/15622975.2013.866693

Radtke, K. M., Ruf, M., Gunter, H. M., Dohrmann, K., Schauer, M., Meyer, A., \& Elbert, T. (2011). Transgenerational impact of intimate partner violence on methylation in the promoter of the glucocorticoid receptor. Transl Psychiatry, 1, e21. doi:10.1038/tp.2011.21

Ramo-Fernandez, L., Schneider, A., Wilker, S., \& Kolassa, I. T. (2015). Epigenetic alterations associated with war trauma and childhood maltreatment. Behav Sci Law, 33(5), 701721. doi:10.1002/bsl.2200

Ratanatharathorn, A., Boks, M. P., Maihofer, A. X., Aiello, A. E., Amstadter, A. B., Ashley-Koch, A. E., ... Smith, A. K. (2017). Epigenome-wide association of PTSD from heterogeneous cohorts with a common multi-site analysis pipeline. Am J Med Genet B Neuropsychiatr Genet, 174(6), 619-630. doi:10.1002/ajmg.b.32568

Reyes, L., \& Manalich, R. (2005). Long-term consequences of low birth weight. Kidney Int Supp/(97), S107-111. doi:10.1111/j.1523-1755.2005.09718.x

Rodney, N. C., \& Mulligan, C. J. (2014). A biocultural study of the effects of maternal stress on mother and newborn health in the Democratic Republic of Congo. Am J Phys Anthropol, 155(2), 200-209. doi:10.1002/ajpa.22568

Rutten, B. P. F., Vermetten, E., Vinkers, C. H., Ursini, G., Daskalakis, N. P., Pishva, E., . . Boks, M. P. M. (2018). Longitudinal analyses of the DNA methylome in deployed military servicemen identify susceptibility loci for post-traumatic stress disorder. Mol Psychiatry, 23(5), 1145-1156. doi:10.1038/mp.2017.120

Sartor, C. E., Grant, J. D., Lynskey, M. T., McCutcheon, V. V., Waldron, M., Statham, D. J., . . . Nelson, E. C. (2012). Common heritable contributions to low-risk trauma, high-risk trauma, posttraumatic stress disorder, and major depression. Archives of General Psychiatry, 69(3), 293-299. doi:10.1001/archgenpsychiatry.2011.1385 
Sartor, C. E., McCutcheon, V. V., Pommer, N. E., Nelson, E. C., Grant, J. D., Duncan, A. E., . . Heath, A. C. (2011). Common genetic and environmental contributions to posttraumatic stress disorder and alcohol dependence in young women. Psychol Med, 41(7), 1497-1505. doi:10.1017/S0033291710002072

Stein, M. B., Jang, K. L., Taylor, S., Vernon, P. A., \& Livesley, W. J. (2002). Genetic and environmental influences on trauma exposure and posttraumatic stress disorder symptoms: a twin study. Am J Psychiatry, 159(10), 1675-1681. doi:10.1176/appi.ajp.159.10.1675

True, W. R., Rice, J., Eisen, S. A., Heath, A. C., Goldberg, J., Lyons, M. J., \& Nowak, J. (1993). A twin study of genetic and environmental contributions to liability for posttraumatic stress symptoms. Arch Gen Psychiatry, 50(4), 257-264. doi:10.1001/archpsyc.1993.01820160019002

Vinkers, C. H., Kalafateli, A. L., Rutten, B. P., Kas, M. J., Kaminsky, Z., Turner, J. D., \& Boks, M. P. (2015). Traumatic stress and human DNA methylation: a critical review. Epigenomics, 7(4), 593-608. doi:10.2217/epi.15.11

Waterland, R. A., \& Jirtle, R. L. (2003). Transposable elements: targets for early nutritional effects on epigenetic gene regulation. Mol Cell Biol, 23(15), 5293-5300. doi:10.1128/mcb.23.15.5293-5300.2003

Xian, H., Chantarujikapong, S. I., Scherrer, J. F., Eisen, S. A., Lyons, M. J., Goldberg, J., . . . True, W. R. (2000). Genetic and environmental influences on posttraumatic stress disorder, alcohol and drug dependence in twin pairs. Drug \& Alcohol Dependence, 61(1), 95-102. doi:10.1016/S0376-8716(00)00127-7

Yehuda, R., \& Bierer, L. M. (2008). Transgenerational transmission of cortisol and PTSD risk. Prog Brain Res, 167, 121-135. doi:10.1016/S0079-6123(07)67009-5

Yehuda, R., Daskalakis, N. P., Bierer, L. M., Bader, H. N., Klengel, T., Holsboer, F., \& Binder, E. B. (2016). Holocaust exposure induced intergenerational effects on FKBP5 methylation. Biol Psychiatry, 80(5), 372-380. doi:10.1016/j.biopsych.2015.08.005 


\section{Chapter 4 Epigenome-Wide Association of PTSD From Heterogeneous Cohorts With a Common Multi-Site Analysis Pipeline}

Andrew Ratanatharathorn ${ }^{1 *}$, Marco P Boks ${ }^{2 *}$, Adam X Maihofer ${ }^{3,4}$, Allison E Aiello ${ }^{5}$, Ananda B Amstadter $^{6}$, Allison E Ashley-Koch7, Dewleen G Baker ${ }^{3,4}$, Jean C Beckham ${ }^{8,9,10}$, Evelyn Bromet ${ }^{11}$, Michelle Dennis ${ }^{8,10}$, Sandro Galea ${ }^{12}$, Melanie E Garrett ${ }^{7}$, Elbert Geuze ${ }^{2,13}$, Guia Guffanti ${ }^{14,15}$, Michael A Hauser ${ }^{7}$, Varun Kilaru ${ }^{16}$, Nathan A Kimbrel ${ }^{8,9,10}$, Karestan C Koenen ${ }^{17,18,19}$, Pei-Fen Kuan $^{20}$, Mark W Logue ${ }^{21,22}$, Benjamin J. Luft ${ }^{11}$, Mark W MIller ${ }^{22,23}$, Colter Mitchell ${ }^{24}$, Nicole R Nugent $^{25}$, Kerry J Ressler ${ }^{14,15,26}$, Bart P F Rutten ${ }^{27}$, Murray B Stein ${ }^{3,4}$, Eric Vermetten ${ }^{2,13}$, Christiaan H Vinkers ${ }^{2}$, Derek E Wildman ${ }^{28,29}$, Nagy A. Youssef ${ }^{30}$, VA Mid-Atlantic MIRECC Workgroup ${ }^{8}$, PGC PTSD Epigenetics Workgroup, Monica Uddin ${ }^{28,31}$, Caroline M Nievergelt ${ }^{3,4}$, Alicia K Smith ${ }^{16}$

${ }^{1}$ Columbia University, Department of Epidemiology

${ }^{2}$ University Medical Center Utrecht, Brain Center Rudolf Magnus

${ }^{3}$ University of California San Diego, Department of Psychiatry and Department of Family Medicine and Public Health

${ }^{4}$ Veterans Affairs San Diego Healthcare System and Veterans Affairs Center of Excellence for Stress and Mental Health ${ }^{5}$ UNC Chapel Hill, Department of Epidemiology

${ }^{6}$ Virginia Commonwealth University, Department of Psychiatry

${ }^{7}$ Duke University, Center for Human Disease Modeling

${ }^{8}$ VA Mid-Atlantic, Mental Illness Research, Education, and Clinical Center

${ }^{9}$ Durham VA Medical Center

${ }^{10}$ Duke University Medical Center, Department of Psychiatry and Behavioral Sciences

${ }^{11}$ Stony Brook University, Departments of Psychiatry and Preventive Medicine

${ }^{12}$ Boston University School of Public Health

${ }^{13}$ Military Mental Healthcare- Research Centre, Ministry of Defense

${ }^{14}$ McLean Hospital, Neurobiology of Fear Laboratory

${ }^{15}$ Harvard T.H. Chan School of Public Health, Department of Epidemiology and Massachusetts General Hospital, Department of Psychiatry

${ }^{16}$ Emory University, Department of Gynecology and Obstetrics

${ }^{17}$ Harvard T.H. Chan School of Public Health, Department of Epidemiology

${ }^{18}$ Massachusetts General Hospital, Psychiatric and Neurodevelopmental Genetics Unit, Center for Human Genetic Research, and Department of Psychiatry

${ }^{19}$ Broad Institute of MIT and Harvard, Stanley Center for Psychiatric Research

${ }^{20}$ Stony Brook University, Department of Applied Mathematics and Statistics

${ }^{21}$ Boston University, Department of Medicine (Biomedical Genetics)

${ }^{22} V A$ Boston Healthcare System

${ }^{23}$ Boston University School of Medicine, Department of Psychiatry 
${ }^{24}$ University of Michigan, Institute for Social Research

${ }^{25}$ Brown University, Psychiatry and Human Behavior, Department of Pediatric Research

${ }^{26}$ Emory University, Department of Psychiatry

${ }^{27}$ Maastricht University Medical Centre, School for Mental Health and Neuroscience, Department of Psychiatry and Neuropsychology

${ }^{28}$ University of Illinois Urbana-Champaign, Carl R. Woese Institute for Genomic Biology

${ }^{29}$ University of Illinois Urbana-Champaign, Department of Molecular \& Integrative Physiology

${ }^{30}$ Medical College of Georgia at Augusta University, Department of Psychiatry and Human

Behavior and Office of Academic Affairs

${ }^{31}$ University of Illinois Urbana-Champaign, Department of Psychology

* Indicates equal contribution

Published as: Ratanatharathorn A, Boks MP, Maihofer AX, Aiello AE, Amstadter AB, Ashley-Koch AE, Baker DG, Beckham JC, Bromet E, Dennis M, Garrett ME, Geuze E, Guffanti G, Hauser MA, Kilaru V, Kimbrel NA, Koenen KC, Kuan PF, Logue MW, Luft BJ, Miller MW, Mitchell C, Nugent $N R$, Ressler KJ, Rutten BPF, Stein MB, Vermetten E, Vinkers CH, Youssef NA; VA Mid-Atlantic MIRECC Workgroup; PGC PTSD Epigenetics Workgroup, Uddin $M$, Nievergelt CM, Smith AK. Epigenome-wide association of PTSD from heterogeneous cohorts with a common multi-site analysis pipeline. Am J Med Genet B Neuropsychiatr Genet. 2017 Sep;174(6):619-630. doi: 10.1002/ajmg.b.32568. Epub 2017 Jul 10. PubMed PMID: 28691784; PubMed Central PMCID: PMC5592721.

https://onlinelibrary.wiley.com/doi/full/10.1002/ajmg.b.32568 


\section{$\underline{\text { Abstract }}$}

Compelling evidence suggests that epigenetic mechanisms such as DNA methylation play a role in stress regulation and in the etiology of stress-related disorders such as Post-Traumatic Stress Disorder (PTSD). Here we describe the purpose and methods of an international consortium that was developed to promote collaborative studies on the role of epigenetics in PTSD. Inspired by the approach used in the Psychiatric Genomics Consortium, we brought together investigators representing seven cohorts (total $\mathrm{N}=1147$ ) that included detailed information on trauma exposure, PTSD symptomatology, and genome-wide DNA methylation. The objective of this consortium is to increase the analytical sample size by pooling data and combining expertise in order to identify the DNA methylation patterns associated with PTSD. Several quality control and analytical pipelines were evaluated for their control of genomic inflation and technical artifacts, and a joint analysis procedure was established to derive comparable data over the cohorts for meta-analysis. We propose ways to deal with ancestry population stratification and type I error inflation and discuss the advantages and disadvantages of applying robust error estimates. To evaluate our pipeline, we report results from an epigenome-wide association study (EWAS) of age, which is a well-characterized phenotype with known epigenetic associations. Overall, while EWAS are highly complex and subject to similar challenges as genome-wide association studies (GWAS), we demonstrate that an epigenetic meta-analysis can be well-powered to identify epigenetic associations. This study can be used as a framework for consortium efforts for EWAS.

\section{Keywords}

EWAS, meta-analysis, trauma 


\section{INTRODUCTION}

Traumatic events are reported by over $70 \%$ of individuals during their lifetime (Benjet et al., 2016). They have been associated with a number of deleterious outcomes, including posttraumatic stress disorder (PTSD), a psychiatric disorder characterized by intrusions, avoidance, negative alterations in thoughts and mood, and alterations in physiological arousal and reactivity (American Psychiatric Association, 2000). PTSD can be severe and disabling and is often associated with a range of comorbid psychiatric conditions, such as depression and substance use disorders (Brady, Killeen, Brewerton, \& Lucerini, 2000; Najt, Fusar-Poli, \& Brambilla, 2011). PTSD has also been associated with a 2.8-fold increase in suicidal thoughts and behaviors (Sareen, Houlahan, Cox, \& Asmundson, 2005) as well as with a number of chronic medical conditions (Boscarino, 2008; Coughlin, 2011; David, Woodward, Esquenazi, \& Mellman, 2014; Heppner et al., 2009; Jakovljevic et al., 2008; Kubzansky, Koenen, Jones, \& Eaton, 2009; Kubzansky, Koenen, Spiro, Vokonas, \& Sparrow, 2007). While the risk of developing PTSD depends, in part, on the nature of the traumatic event (Kessler, 2000), only a minority of those exposed to trauma develop PTSD. As a result, despite the high prevalence of lifetime trauma, the overall lifetime prevalence of PTSD in the United States is 6.8\%, (Breslau et al., 1998; Kessler et al., 2005; Resnick, Kilpatrick, Dansky, Saunders, \& Best, 1993), which suggests there are individual differences in resilience that, if better understood, might inform the development of new approaches to prevention and treatment.

Genetic epidemiological studies suggest that both genetic and environmental factors contribute to PTSD risk. Twin studies estimate the heritability of PTSD to be between 30-70\% (Sartor et al., 2012; Sartor et al., 2011; Stein, Jang, Taylor, Vernon, \& Livesley, 2002; True et al., 1993; Xian et al., 2000), with the remaining variance being attributed to environmental factors. Genetic research, both candidate gene and genome-wide association studies (GWAS), provided support for the role of genetics in the development and severity of PTSD and have begun to identify variants that account for some of the genetic influence on PTSD. The genetic loci identified in the extant GWAS have been implicated in a variety of processes, including neuroprotection, actin polymerization, neuronal function, and immune function (Almli et al., 2014; Guffanti et al., 2013; Logue et al., 2013; Xie et al., 2013). Although promising, no robust genetic variants associated with PTSD have been identified, and much work remains to be done to understand the biological basis of PTSD risk (Logue et al., 2015).

A growing body of work has explored the role of environmental influences on an individual's response to trauma. Clarifying the ways in which environmental influences might affect biological function is critical to understanding the etiology of PTSD. In this regard, epigenetic mechanisms, which can mediate environmental influences on gene function, are particularly relevant. Epigenetic modifications, such as DNA methylation at cytosine-guanine dinucleotides (CpG sites), induce changes in gene expression through structural alterations of DNA that are maintained through each round of cell division; they respond to changes in the environment, are potentially reversible, and can be targeted for disease therapies (Feinberg, 
2007). DNA methylation regulates gene expression by influencing the recruitment and binding of regulatory proteins to DNA. Typically, higher methylation at gene promoter regions correlates with decreased expression of that gene, while intragenic methylation can regulate alternative promoters and enhancers (Bonasio, Tu, \& Reinberg, 2010; Maunakea et al., 2010).

For example, animal studies have demonstrated that epigenetic changes-particularly alterations in DNA methylation in response to nurturing-are related to altered responses to stress (Jirtle \& Skinner, 2007; Weaver et al., 2004). Similar alterations have been reported in the human literature, in both central and peripheral tissues (McGowan et al., 2009; Tyrka, Price, Marsit, Walters, \& Carpenter, 2012). Considering the influence of traumatic stress on DNA methylation seen in some studies (Vinkers et al., 2015), epigenetic-based investigations may extend research findings. For example, research reporting an association of PTSD with a genetic variant in the PAC1 receptor (ADCYAP1R1; rs2267735) went beyond this finding to observe that PTSD severity was also correlated with methylation levels of the gene (Ressler et al., 2011). Other work has suggested an interactive effect between traumatic burden and DNA methylation in the serotonin transporter locus (SLC6A4) on PTSD risk, independent of the widely studied length polymorphism at this same locus (Koenen et al., 2011) and a potentially interacting effect of genetic and epigenetic variation of the dopamine receptor (SLC6A3) on PTSD risk (Chang et al., 2012). Indeed, stress exposure itself has been shown to alter epigenetic patterns in both animal and human studies (Moser et al., 2015; Roth, Lubin, Funk, \& Sweatt, 2009; Sipahi et al., 2014). In addition to candidate gene methylation studies, a small number of studies have examined genome-wide methylation patterns in PTSD (Mehta et al., 2013; Smith et al., 2011; Uddin et al., 2010). In these first genome-wide studies of DNA methylation, immune dysregulation figured prominently among the biological networks associated with PTSD and, at a CpG site level, DNA methylation levels in several CpGs showed suggestive evidence of replication between these studies (Mehta et al., 2013; Smith et al., 2011; Uddin et al., 2010).

Although the emerging literature on epigenetic influences on PTSD is promising, the majority of research to date has been conducted with modest sample sizes, with inherent limited statistical power. Furthermore, studies of DNA methylation have been hampered by technical issues including batch effects (Harper, Peters, \& Gamble, 2013) and blood cell composition (Houseman et al., 2012). Experience from large-scale genetic studies, such as the Psychiatric Genomics Consortium (PGC), underscores how large collaborative endeavors can provide the adequate sample sizes and the sufficient statistical power necessary to produce significant and replicable results. Based on this experience, the PGC-PTSD formed the epigenome-wide association study (EWAS) working group to organize an expansive collection of samples with genome-wide methylation data available for joint analyses.

Although there are many advantages to this collaborative approach, there are also challenges. First, assessments of trauma-exposure and PTSD symptoms/diagnosis differ among the cohorts, requiring harmonization of the phenotypic data. The larger PGC-PTSD has made substantial progress harmonizing phenotypes across studies, and a similar approach will be 
adopted by the PGC-PTSD EWAS group in its analyses. A second challenge of consortia is to address complications in the data-sharing that is a prerequisite to increasing the power and reproducibility of the study. The trend in psychiatric genetics has been to establish large-scale consortia for the purpose of expanding sample sizes beyond what is possible based on the data from any one group. Almost universally used in current large-scale genomic consortia is a metaanalysis, through which the data from individual sites are analyzed separately and combined, based on summary statistics. In many cases, meta-analysis of individual-level results yields results comparable to those of a "mega-analysis" of pooled data from different studies (Lin \& Zeng, 2010; Mathew \& Nordstrom, 1999; Olkin \& Sampson, 1998). However, there have been important distinctions in the way the meta-analysis strategy has been implemented across consortia, including variations in the degree to which the cleaning and analysis of the individual-level data occurs (i.e., centralized versus distributed). The cleaning and analysis of individual data in a centralized manner allows a high degree of control over the quality control (QC) process and an ability to quickly perform follow-up analyses, but poses the difficulties of requiring a larger degree of computation and storage at the consortium level and permission from all groups to share data.

The PGC-PTSD uses many of the same protocols and tools developed by the PGC (Logue et al., 2015), with the difference that not all data are centrally stored and managed, since some constituent samples that originate from military, Veteran (VA) or foreign countries are subject to additional regulatory oversight, which do not allow the sharing of individual-level genomic data. To accommodate participation for these studies, the PGC-PTSD EWAS group follows a strategy similar to that of the ENIGMA consortium (Thompson et al., 2014), in which a set of protocols and scripts are created to implement standardized QC and analysis pipelines for the Illumina HumanMethylation450 BeadChip. These scripts are performed at each participating site, and analysis results are submitted to the consortium where they are assessed, collated, and metaanalyzed. In this study, we compare the performance of two QC and two analytical pipelines to control for genomic inflation, present the final PGC-PTSD EWAS pipeline, and assess the performance of the PGC-PTSD EWAS pipeline in a meta-analysis of age.

\section{MATERIALS AND METHODS}

The PGC EWAS Cohorts

The participating cohorts, presented in Table 1, consisted of four military cohorts (MRS, PRISMO, VA-M, and VA-NCPTSD) and three civilian cohorts (DNHS, GTP, and WTC) that all measured DNA methylation (DNAm) with the Illumina HumanMethylation450K BeadChip. Descriptions of the cohorts are in the supplemental information. Each cohort consisted of PTSD cases as well as trauma-exposed controls. A total of 1,147 subjects ( $~ 50 \%$ cases) were selected for inclusion in the EWAS and were subjected to the quality control and analytical pipelines.

Posttraumatic Stress Disorder 
Similar to other analyses conducted by the PGC-PTSD, our analysis required consistently defining and harmonizing PTSD diagnoses across cohorts that used different instruments and methods of diagnosis (Logue et al., 2015). We used a diagnosis of current PTSD based on the diagnostic criteria defined by each cohort's principal investigator (see supplemental information). Individuals with lifetime diagnoses of PTSD but not current PTSD were excluded from analysis.

\section{Quality Control Procedures}

We tested two quality control protocols: the eventually proposed PGC pipeline and a Functional Normalization (Funnorm) pipeline. In the PGC pipeline (Supplemental Figure 1), study investigators first conducted a visual inspection of control-probes designed to report each step of the Infinium protocol such as bisulfite conversion and hybridization efficiency. In addition, samples with probe detection call rates $<90 \%$ and those with an average intensity value of either $<50 \%$ of the experiment-wide sample mean or $<2,000$ arbitrary units (AU) were excluded. Probes with detection $p$-values $>0.001$ or that were based on less than three beads were set to missing as were probes that cross-hybridized between autosomes and sex chromosomes (Teschendorff et al., 2013). CpG sites with missing data for $>10 \%$ of samples within cohorts were excluded from analysis. Probes containing single nucleotide polymorphisms (SNPs, based on 1000 genomes) within 10 base pairs of the target CpG were maintained in each dataset, but flagged and tracked throughout the analysis pipeline. This decision was based on the growing recognition that sequence variants can influence DNA methylation patterns throughout the genome. Considering our aim to identify genes important for PTSD, even if an associated CpG site is influenced by genetics, such as the SKA2, maintaining such probes is informative (M. P. Boks et al., 2016; Gibbs et al., 2010; Guintivano et al., 2014; Heyn et al., 2013; Rice et al., 2008; Smith et al., 2014). Normalization of probe distribution and background differences between Type I and Type II probes was conducted using Beta Mixture Quantile Normalization (BMIQ) (Teschendorff et al., 2013) after background correction. We chose BMIQ after comparing distributions of BMIQ normalized Type II probes in the Detroit Neighborhood Health Study with the raw distributions and distributions after applying the DASEN procedure in the $\mathrm{R}$ package wateRmelon (Supplemental Figure 2) (Pidsley et al., 2013).

Following normalization, batch effect removal, as implemented in the ComBat procedure of the SVA package in bioconductor, was used to account for sources of technical variations including batch and positional effects, which can cause spurious associations (Johnson, Li, \& Rabinovic, 2007). Individual cohorts also controlled for additional covariates that may not have been balanced within chips but that were of interest in downstream analyses, such as case designation and sex (if relevant). Following completion of this QC pipeline, each cohort confirmed that there were no remaining sources of technical variation by examining the association of PCs of the methylation levels with chip and position using multivariate linear regression, bar plots, and heat maps. 
The second QC protocol used the functional normalization procedure implemented in the $R$ package minfi, which has been reported to remove technical variation more effectively than ComBat or other supervised methods (Aryee et al., 2014; Fortin et al., 2014). Briefly, raw IDATs were loaded into $R$ and functional normalization applied using the default of two principal components as covariates. The resulting normalized beta matrix was then extracted and used for analysis.

\section{Cellular Heterogeneity}

DNA methylation is known to vary by cell type, which impacts the measured whole blood methylation as a result of the amalgamation of the cell type proportions in each individual sample. To control for possible confounding by individuals' underlying cell type heterogeneity, proportions of CD8, CD4, NK, B cells, monocytes and granulocytes were estimated using each individual's DNA methylation data, publicly available reference data (GSE36069), and the method described by Jaffe and colleagues (Jaffe \& Irizarry, 2014; Reinius et al., 2012), based on the Houseman algorithm (Houseman et al., 2012). CD8, CD4, NK, B cell, and monocyte cell proportions were included as covariates in our statistical analyses.

\section{Ancestry}

Accounting for population stratification has become routine practice for genetic association studies, and most recently has also been shown to be of importance in DNA methylation studies (Barfield et al., 2014; Nielsen et al., 2010). GWAS methods such as principal components (PCS) derived from single-nucleotide polymorphisms (SNPs) can be incorporated into EWAS, but were not always available for all cohorts, or all samples within a cohort. An alternative based on methylation probes that proxy nearby SNPs was developed by Barfield et al. for use in European and African American subjects (Barfield et al., 2014). Here we evaluated and extended this approach to other ancestral populations as part of the PGC-PTSD EWAS pipeline.

A subset of ancestry-diverse subjects ( $\mathrm{N}=128$, including European Americans, African Americans, Latinos/Native Americans and 'others' including East Asians) from the Marine Resiliency study (MRS) was selected based on available genome-wide genotype data (Illumina HumanOmniExpressExome array) and matching Illumina 450K methylation data (C M Nievergelt et al., 2015). Ancestry using GWAS data was inferred as described in Nievergelt et al. (C. M. Nievergelt et al., 2013). In brief, genotypes of 1783 ancestry-informative markers (AIMs) were used to determine a subject's ancestry at the continental level using STRUCTUREv2.3.2.1, including prior population information of the HGDP reference set (Falush, Stephens, \& Pritchard, 2003; Li et al., 2008). Based on these ancestry estimates, subjects were placed into one of 4 groups: European Americans, African Americans, Latinos/Native Americans and 'Others'. Principal components (PCs) were derived using Eigenstrat (Price et al., 2006).

Ancestry estimates using methylation data were derived using subsets of methylation probes in close proximity to SNPs identified by Barfield et al. (Barfield et al., 2014). Probe sets with Obp distance ( $N=7,703 \mathrm{CpG}$ probes), within 1 bp distance $(\mathrm{N}=17,995 \mathrm{CpG}$ probes), and within 
10bp distance ( $\mathrm{N}=50,319$ CpG probes) were compared. GWAS-derived PCs were visually compared to methylation-probe derived PCs, and genotypes of SNPs in proximity of CpG sites were compared with respective CpG methylation values using Pearson correlation ( $r$ ).

\section{Statistical Analysis}

Within each cohort, logit transformed ß values (M-values) (Du et al., 2010) were modeled by linear regression as a function of PTSD, adjusting for sex, age, the estimated cell proportions, and ancestry using the either the first three PCs from GWAS data if available or the second through fourth PCs from EWAS data. QQ-plots of the PTSD p-values were examined for evidence of genomic inflation due to unaccounted technical variation or other confounders. In addition, the genomic inflation factors $(\lambda)$ were calculated for each study. Two adjustments were considered to improve the precision of the estimated variances. First, moderated t-statistics were calculated using the empirical Bayes method implemented in the R package limma (Smyth, 2005). Second, HC3 robust standard errors, which have been shown to be the most effective in samples smaller than 250, were calculated using the R package sandwich (Long \& Ervin, 2000; Zeileis, 2004).

Cohort-level analysis results were combined using the inverse normal method (Marot, Foulley, Mayer, \& Jaffrézic, 2009). Briefly, one-sided p-values for each CpG site in each study were calculated from the t-statistics. Next, z-scores were calculated from the one-sided $p$-values and weighted by the number of subjects in each study relative to the total in the meta-analysis. Twosided $p$-values of the $z$-score were then calculated, and genomic inflation examined. Finally, $p$ values were adjusted for multiple-testing using the False Discovery Rate (FDR) procedure at the Type I error rate level of 5 percent (Benjamini \& Hochberg, 1995).

\section{Sensitivity Analysis}

We also conducted a sensitivity analysis by modeling methylation as a function of age along with covariates for gender and ancestry and meta-analyzed as outlined above except without using an empirical Bayes adjustment to simplify our analysis in order to help identify statistical issues that may cause inflation or deflation of our results. Numerous robust associations between age and DNAm have been reported, and we examined whether the increased sample size in the meta-analysis increased the number of significant CpG sites over the individual studies (Bocklandt et al., 2011; Hannum et al., 2013; Horvath, 2013; Horvath et al., 2012; Weidner et al., 2014). In addition, we measured concordance between studies by estimating the correlation between the t-statistics of the age variable calculated without either standard error adjustment.

Power

Power for EWAS is more favorable compared to GWAS as a result of the continuous nature of the DNA methylation measures, but instead suffers from poor distribution including low variances and heteroscedasticity. The power to detect a differentially methylated CpG site 
depends on the percent difference in methylation between cases and controls, the pooled variation in methylation ( $\sigma$ ) across CpG sites, and the number of cases and controls (Liu \& Hwang, 2007). We conducted simulations to test the smallest mean difference between PTSD cases and controls we could detect based on our projected sample size and a $\sigma=0.43$, which represents the highest variation reported in one of our cohorts, and thus a very conservative estimate (Orr \& Liu, 2009).

\section{RESULTS}

\section{Participating Cohorts}

Sample characteristics for studies that have contributed data to this first PGC-PTSD EWAS study are listed in Table 1 ( $\mathrm{N}=1147)$. Three of the seven studies are composed of civilians, while the remaining studies include active duty and veteran military populations. The majority of participants, especially from the military cohorts, were male (73\%) and of European American (EA) descent (56\%). All participants were exposed to trauma, and half suffered from PTSD (50\%). Data collection occurred across the United States (e.g., Atlanta, Detroit, San Diego, Durham, Boston) and Europe. While a few studies used clinical interviews, the majority of studies used self-report ratings of PTSD symptoms. A detailed description of participating cohorts is provided in the supplementary information.

Power

The power analysis shows that with our sample of 573 cases and 574 controls $(N=1147)$, we are sufficiently powered to find at least one CpG site with a mean methylation difference of 0.08 between cases and controls (Figure 1 ).

Ancestry

We investigated the utility of DNA methylation-based ancestry estimates based on CpGs with nearby genetic variants in cis as proposed by Barfield et al. (Barfield et al., 2014). A comparison of $\mathrm{CpG}$ probes with SNPs within $1 \mathrm{bp}$ distance $(\mathrm{N}=17,995)$ and $\mathrm{CpG}$ probes with SNPs within $10 \mathrm{bp}$ distance $(\mathrm{N}=50,319)$ showed a higher genotype-methylation correlation for the $1 \mathrm{bp}$ probes $\left(r=0.29, p=1.8 \times 10^{-15}\right)$ than the $10 b p$ probes $(r=0.06, p=0.0015)$. Figure 2 shows a SNPderived PC plot based on available GWAS data including PC1 and PC2 (panel A), PCs 2 and 3 from the methylation-based CpGs with a SNP 1 bp from the probes (panel B) and the PCs from CpG probes within 10bp of a SNP (panel C). Supplemental Figure S5 shows PCs 2, 3, 4, and PCs 2, 3, and 6 for, respectively, CpGs within $1 \mathrm{bp}$ and $10 \mathrm{bp}$ of a SNP. These results along with the genotype-methylation correlations show that the use of DNA methylation ancestry estimates (PCs 2-4) using probes within $1 \mathrm{bp}$ of a SNP provide reliable results and are suitable as ancestry covariates in our analyses.

Quality Control Results 
The number of samples and probes, not including cross-reactive probes, removed in our proposed PGC-EWAS pipeline ranged from 677 to 10,218 across studies (Supplemental Table S3). Figure 3 presents the genomic inflation factors from the analysis of PTSD for each individual study using two different quality control methods as well as two different analysis pipelines: (1) our proposed PGC-EWAS pipeline, described in detail in the Methods section above, as well as (2) the Functional Normalization (Funnorm) QC pipeline. Resulting data from each QC pipeline were then subjected to linear regression analysis, performed with empirical Bayes and HC3 standard errors, respectively. In studies using the Funnorm pipeline there were large variations in the genomic inflation factor with two studies showing high inflation (DNHS, GTP) and two studies showing substantial deflation (MRS, VA-NCP) regardless of whether empirical Bayes or HC3 standard errors were used. Using the PGC-EWAS pipeline and HC3 standard errors, six of the seven studies showed genomic deflation with $\lambda<1.0$, while only one study was deflated when using empirical Bayes standard errors (Supplemental Table S1). These results indicate that the PTSD-PGC EWAS pipeline, combined with empirical Bayes standard errors, is the preferred method for cohorts participating in our meta-analysis.

\section{Sensitivity Analysis: Age Meta-Analysis Results}

Results for the age analysis using our pipeline and no standard error adjustment are presented in Table 3. All studies reported numerous FDR-significant CpG sites but substantially more significant results were reported for the combined meta-analysis. MRS and PRISMO reported the fewest significant sites. However, the age range for participants in these studies was narrower as both studies recruited active military personnel. The correlations of the $\mathrm{t}$ statistics ranged from 0.171 to 0.692 when all sites were analyzed and from 0.441 to 0.886 among the FDR significant sites (Supplemental Figure 3). The strong correlations of the most significant sites indicate that each cohort retained the biological signal of age after QC. Of the 494 CpG sites reported to have been associated with age, 326 were significant after FDR-correction (Supplemental Table 2). In addition, a forest plot of the most significant CpG site, representative of the FDR significant sites, shows a consistent direction of effect in each study (Supplemental Figure 4).

\section{DISCUSSION}

PTSD is unique among psychiatric disorders in that its diagnosis requires exposure to a significant traumatic event. With an environmental exposure embedded into the etiology of the disorder, the PTSD diagnosis affords an unusual opportunity to identify individual differences in the biological response to trauma to increase risk for, or resilience to, the disorder. Here we have introduced an international collaboration that has been established to identify epigenetic associations--specifically, DNA methylation--that are related to risk for, or resilience to, PTSD. We presented the development of a consistent pipeline for processing and quality control of epigenome-wide association data comparing two quality control approaches and statistical 
pipelines. In our analysis of PTSD, we found that our proposed PGC-EWAS pipeline controlled for genomic inflation and deflation more consistently than functional normalization, regardless of the standard error correction used. In light of these findings, we encourage consortia to implement our quality control pipeline including checks for genomic inflation and strengthened associations with age before meta-analyzing across studies.

Through these collaborative efforts to analyze existing DNA methylation data from blood obtained from both military and civilian cohorts, we are poised to collectively address one of the main challenges of psychiatric genomics, namely the need for large, harmonized samples to adequately power genome-scale analyses. The current collaborative dataset allows detection of methylation differences around seven percent, larger than most reported methylation differences (Vinkers et al., 2015). Additional EWAS datasets that are forthcoming will likely prove essential to detecting PTSD-associated DNA methylation differences in our planned metaanalyses. Moving forward, we anticipate that our collaborative efforts will grow to include additional cohorts from around the world; indeed, in the last year alone, several new studies have expressed interest in participating in future EWAS analyses as their data become available. In addition to the DNA methylation analysis, the close allegiance with the PGC-PTSD group has laid the foundation for integrating data from genome-wide association (GWAS), EWAS, and gene expression/transcriptome analyses. In combination with other biological measures and coordinated neuroimaging efforts (Logue et al., 2015) that may become accessible through this collaboration, these system-wide integrations will facilitate a more complete understanding of the molecular architecture and biological underpinnings of PTSD.

The harmonization of some study characteristics paired with the demographic and clinical diversity of the samples, including the differences between military and civilian trauma, allows us the opportunity to identify DNA methylation patterns predictive for specific groups of individuals and types of trauma. This will not only provide insight into the heterogeneity of PTSD, but may also help explain mechanisms for the variation in conditional effects of different types of trauma on PTSD (Wisco et al., 2014). Additionally, it will also provide a framework from which DNA methylation may be informative for early prediction and treatment.

Looking ahead, we are optimistic that our PTSD EWAS collaboration will identify bloodbased DNA methylation signatures that associate reliably with PTSD. Identification of robust peripheral biomarkers is an important first step and has potential for early detection and prevention. The ultimate goal is to provide new insights into the etiology of PTSD. To truly understand the mechanistic basis of PTSD, it will be critical to compare our blood-derived epigenetic biomarkers with those from other tissues, in particular brain tissue. As a first pass, DNA methylation-based biomarkers that associate with PTSD at particular CpG sites in blood can, at this time, be compared to CpG site derived from brain tissues, thanks to the Epigenomic Roadmap datasets (Bernstein et al., 2010; Kundaje et al., 2015). However, these comparisons will be limited to a comparison of DNA methylation levels in brain and blood in general, as the current data are not representative of PTSD. Over time, however, the development of PTSD brain 
biobanks of brain and other tissue types, including blood cells, will help us to pinpoint whether blood-derived, DNA methylation biomarkers of PTSD reflect similar alterations in brain tissue. Recent work suggests that this is possible for certain pathways (Daskalakis, Cohen, Cai, Buxbaum, \& Yehuda, 2014). Collectively, these cross-tissue efforts will provide insight into the biological pathways underlying PTSD vulnerability and will ultimately facilitate new treatment and prevention. 


\section{Acknowledgements}

This work was supported by the U.S. Army Medical Research and Materiel Command and the National Institute of Mental Health ( $\mathrm{NIMH}$; R01MH108826) as well as the Biomedical and Laboratory Research and Development (\#I01BX002577).

We appreciate the technical support of all of the staff, volunteers and participants from the Grady Trauma Project, supported by the National Institutes of Mental Health (MH096764 and MH071537).

DNHS was funded by NIH Awards R01DA022720, R01DA022720-S1, and RC1MH088283 to Allison E. Aiello. We are grateful to all of the participants and staff for their contributions to the DNHS.

The Marine Corps, Navy Bureau of Medicine and Surgery (BUMED) and VA Health Research and Development (HSR\&D) provided funding for MRS data collection and analysis (PI DGB) and NIH R01MH093500 funded the GWAS assays and analysis (PI CMN). Acknowledged are Victoria B. Risbrough Ph.D (VA San Diego Healthcare System \& UCSD), Mark A. Geyer (UCSD), Daniel T. O'Connor (UCSD), all MRS investigators, and all MRS investigators, as well as the MRS administrative core and data collection staff listed in the Methods article (Baker et al, Prev Chronic Dis. 2012;9(10):E97). The authors also thank the Marine and Navy Corpsmen volunteers for military service and participation in MRS.

The data collection of PRISMO was funded by the Dutch Ministry of Defence, and the DNA methylation analyses were funded by the VENI Award fellowship from the Netherlands Organisation for Scientific Research (NWO, grant number 916.11.086) to B.P.F. Rutten.

The VA Boston-National Center for PTSD Study research was supported in part by National Institute of Mental Health Award RO1MH079806 (MWM), Department of Veterans Affairs, Clinical Science Research \& Development Program Award 5I01CX000431-02 (MWM), Department of Veterans Affairs, Biomedical Laboratory Research \& Development Program Award 1I01BX002150-01 (MWM), the Translational Research Center for TBI and Stress Disorders (TRACTS), a VA Rehabilitation Research and Development Traumatic Brain Injury Center of Excellence (B9254-C), and the Cooperative Studies Program, Department of Veterans Affairs. This research is the result of work supported with resources and the use of facilities at the Pharmacogenomics Analysis Laboratory, Research and Development Service, Central Arkansas Veterans Healthcare System, Little Rock, Arkansas. This work was also supported by a Career 
Development Award to E. J. Wolf from the Department of Veterans Affairs, Clinical Sciences Research, and Development Program.

Service of the Department of Veterans Affairs' Office of Research and Development (VA ORD). Dr. Kimbrel was supported by a Career Development Award (\#IK2CX000525) from the Clinical Science Research and Development (CSR\&D) Service of VA ORD. Dr. Beckham was supported by a Research Career Scientist Award (\#11S-RCS-009) from the CSR\&D Service of VA ORD. This research was also supported, in part, by a Merit Award (\#I01BX002577) to Drs. Beckham and Hauser from the Biomedical Laboratory Research and Development (BLR\&D) Service of VA ORD.

The VA Mid-Atlantic Mental Illness Research, Education, and Clinical Center Workgroup includes John A. Fairbank, Mira Brancu, Patrick S. Calhoun, Eric A. Dedert, Eric B. Elbogen, Kimberly T. Green, Robin A. Hurley, Angela C. Kirby, Jason D. Kilts, Christine E. Marx, Gregory McCarthy, Scott D. McDonald, Marinell Miller-Mumford, Scott D. Moore, Rajendra A. Morey, Jennifer C. Naylor, Treven C. Pickett, Jared Rowland, Jennifer J. Runnals, Cindy Swinkels, Steven T. Szabo, Katherine H. Taber, Larry A. Tupler, Elizabeth E. Van Voorhees, H. Ryan Wagner, Richard D. Weiner, and Ruth Yoash-Gantz.

The views expressed in this article are those of the authors and do not necessarily reflect the position or policy of the VA, NIMH, or the United States government.

\section{Conflict of Interest}

Wiley-Blackwell requires that all authors disclose any potential sources of conflict of interest. Any interest or relationship, financial or otherwise, that might be perceived as influencing an author's objectivity is considered a potential source of conflict of interest. These must be disclosed when directly relevant or indirectly related to the work that the authors describe in their manuscript. Potential sources of conflict of interest include but are not limited to patent or stock ownership, membership of a company board of directors, membership of an advisory board or committee for a company, and consultancy for or receipt of speaker's fees from a company. The existence of a conflict of interest does not preclude publication in this journal.

If the authors have no conflict of interest to declare, they must also state this.

Dr. Youssef's disclosures include Speaker CME honoraria from the Georgia Department of Behavioral Health and Developmental Disabilities (DHBDD). Dr. Stein has in the past 3 years 
received payments for editorial work from UpToDate, Biological Psychiatry, and Depression and Anxiety. He has also in the past 3 years been paid as a consultant for Actelion Pharmaceuticals, Janssen, Pfizer, Resilience Therapeutics, and Tonix Pharmaceuticals. Dr. Youssef received research support from the Department of Veteran Affairs and The Augusta Biomedical Research Corporation. 
Table 1: PGC-PTSD EWAS Cohorts

\begin{tabular}{|c|c|c|c|c|c|c|c|c|}
\hline & \multirow[b]{2}{*}{ Total } & \multicolumn{3}{|c|}{ Civilian } & \multicolumn{4}{|c|}{ Military } \\
\hline & & DNHS & GTP & WTC & MRS & PRISMO & VA-M & VA-NCP \\
\hline $\mathrm{N}$ & 1147 & 100 & 270 & 180 & 126 & 62 & 176 & 233 \\
\hline $\begin{array}{l}\text { Mean } \\
\text { Age (SD) }\end{array}$ & $\begin{array}{l}38.0 \\
(-)\end{array}$ & $\begin{array}{l}53.6 \\
(14.0)\end{array}$ & $\begin{array}{l}41.7 \\
(12.4)\end{array}$ & $\begin{array}{l}49.7 \\
(8.3)\end{array}$ & $\begin{array}{l}22.2 \\
(3.0)\end{array}$ & $\begin{array}{l}27.1 \\
(9.2)\end{array}$ & $\begin{array}{l}34.9 \\
(9.9)\end{array}$ & $\begin{array}{l}32.0 \\
(8.4)\end{array}$ \\
\hline $\begin{array}{l}\text { Current } \\
\text { PTSD, \% }\end{array}$ & $50 \%$ & $40 \%$ & $39 \%$ & $47 \%$ & $50 \%$ & $50 \%$ & $49 \%$ & $69 \%$ \\
\hline Male, \% & $73 \%$ & $38 \%$ & $30 \%$ & $100 \%$ & $100 \%$ & $100 \%$ & $78 \%$ & $90 \%$ \\
\hline Race, \% & & & & & & & & \\
\hline White & $56 \%$ & $15 \%$ & $5 \%$ & $76 \%$ & $57 \%$ & $100 \%$ & $100 \%$ & 74\% \\
\hline Hispanic & $6 \%$ & $0 \%$ & $0 \%$ & $0 \%$ & $25 \%$ & $0 \%$ & $0 \%$ & $14 \%$ \\
\hline Black & $33 \%$ & $85 \%$ & $94 \%$ & $4 \%$ & $8 \%$ & $0 \%$ & $0 \%$ & $9 \%$ \\
\hline Asian & $1 \%$ & $0 \%$ & $0 \%$ & $0 \%$ & $3 \%$ & $0 \%$ & $0 \%$ & $2 \%$ \\
\hline Other & $3 \%$ & $0 \%$ & $0 \%$ & $20 \%$ & $0 \%$ & $0 \%$ & $0 \%$ & $0 \%$ \\
\hline
\end{tabular}


Table 2: Age Results using PGC QC and Analysis pipeline

\begin{tabular}{|l|l|l|l|l|l|}
\hline Study & Sites & Sites with & Sites with & Sites with & Sites with \\
\hline DNHS & (FDR $<\mathbf{0 . 0 5 )}$ & $\left(\mathbf{p}<5 \times 10^{-5}\right)$ & $\left(\mathbf{p}<5 \times \mathbf{1 0}^{-6}\right)$ & $\left(\mathbf{p}<\mathbf{5 \times 1 0 ^ { - 7 } )}\right.$ \\
\hline GTP & 455,079 & 4,766 & 1,744 & 678 & 299 \\
\hline MRS & 453,351 & 59,100 & 21,562 & 14,299 & 9,586 \\
\hline PRISMO & 446,688 & 246 & 311 & 99 & 34 \\
\hline VA-M & 455,641 & 42,474 & 12,913 & 7,213 & 4,159 \\
\hline VA-NCPTSD & 453,747 & 35,217 & 10,522 & 6,331 & 3,991 \\
\hline WTC & 455,340 & 14,239 & 5,013 & 2,730 & 1,525 \\
\hline Meta-Analysis & 444,164 & 119,308 & 57,332 & 46,629 & 38,656 \\
\hline
\end{tabular}




\section{Figures}

Figure 1: Sample size vs. power to detect differentially methylated $\mathrm{CpG}$ sites. The black curve indicates the number of cases and controls necessary to find a differentially methylated if only one $\mathrm{CpG}$ site exists, while the grey line indicates the size necessary if 10 differentially methylated sites exist.

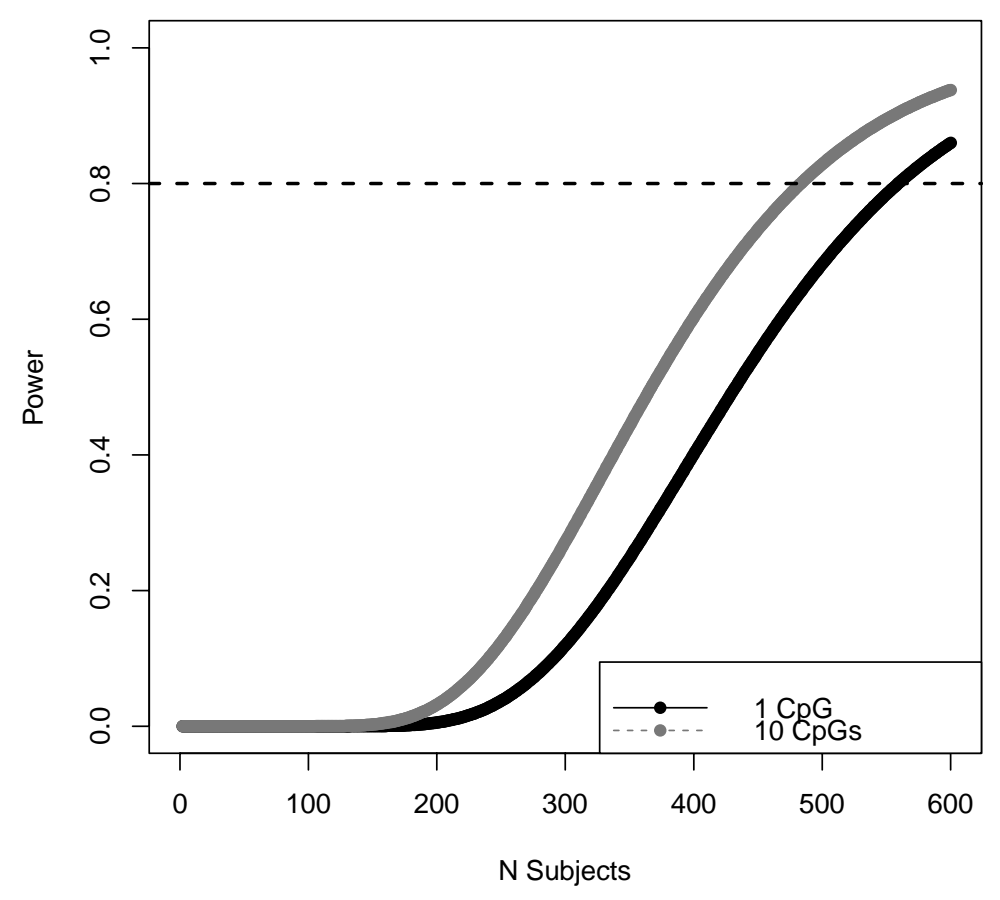


Figure 2: PTSD genomic inflation factors $(\lambda)$ by quality control pipeline (PGC vs. Funnorm) and standard error adjustment method (empirical Bayes vs. HC3).

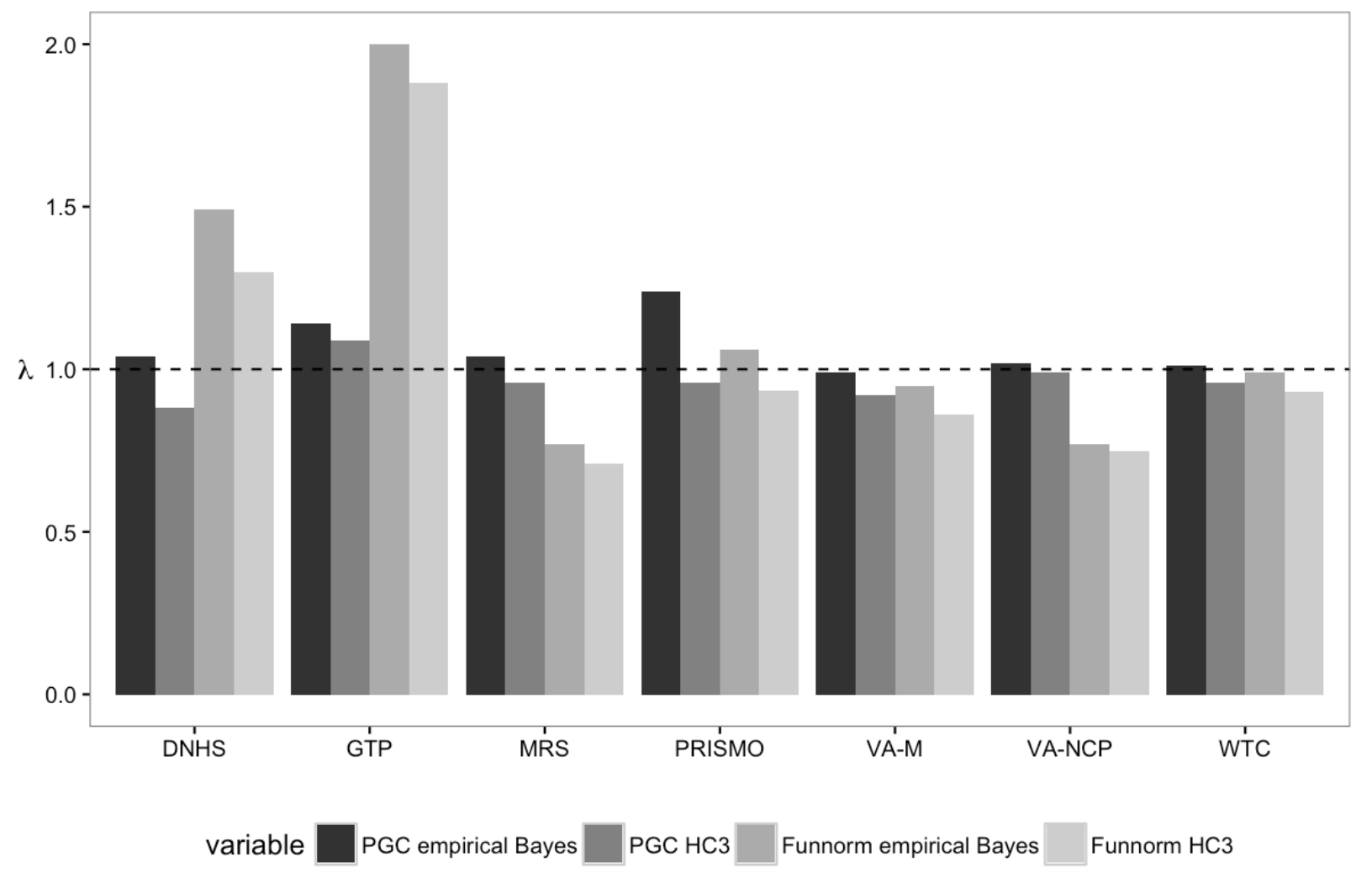


Figure 3: Ancestry inference using SNPs vs. methylation probes in 128 participants of the Marine Resiliency Study (MRS). (A) Principal component (PC) plot showing ancestry inferred using SNPs from a genome-wide association study (GWAS). PC plots based on CpG probes with SNPs within $1 \mathrm{bp}$ distance (B) and with SNPs within $10 \mathrm{bp}$ distance (C), respectively. Subject are placed into 4 ancestral groups based on ancestry estimates using ancestry-informative SNPs and a reference panel (see methods).
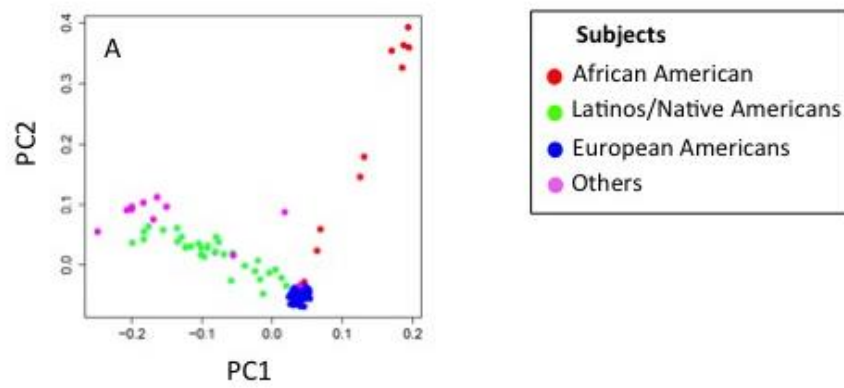

- European Americans

- Others
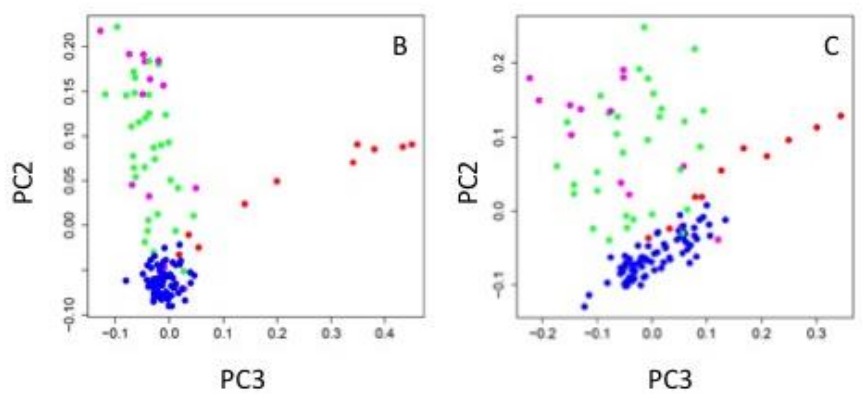
Supplemental Information

Supplemental Table 1: PTSD Genomic Inflation by Quality Control Pipeline

\begin{tabular}{|l|l|l|l|l|l|l|}
\hline Study & $\begin{array}{l}\text { PGC } \\
\text { Sites }\end{array}$ & $\begin{array}{l}\text { PGC } \\
\text { empirical } \\
\text { Bayes }\end{array}$ & $\begin{array}{l}\text { PGC } \\
\text { Pipeline } \\
\text { HC3 }\end{array}$ & $\begin{array}{l}\text { Funnorm } \\
\text { N Sites }\end{array}$ & $\begin{array}{l}\text { Funnorm } \\
\text { empirical } \\
\text { Bayes }\end{array}$ & $\begin{array}{l}\text { Funnorm } \\
\text { HC3 }\end{array}$ \\
\hline DNHS & 455,079 & 1.04 & 0.88 & 485,512 & 1.49 & 1.30 \\
\hline GTP & 453,351 & 1.14 & 1.09 & 485,512 & 2.00 & 1.88 \\
\hline MRS & 455,601 & 1.04 & 0.96 & 485,512 & 0.77 & 0.71 \\
\hline PRISMO & 446,688 & 1.24 & 0.96 & 485,512 & 1.06 & 0.93 \\
\hline VA-M & 455,641 & 0.99 & 0.92 & 485,512 & 0.95 & 0.86 \\
\hline VA-NCP & 453,747 & 1.02 & 0.99 & 485,512 & 0.77 & 0.75 \\
\hline WTC & 455,340 & 1.01 & 0.96 & 485,512 & 0.99 & 0.93 \\
\hline
\end{tabular}


Supplemental Figure 2: Comparison of Type-II probe normalizations in the Detroit Neighborhood Health Study

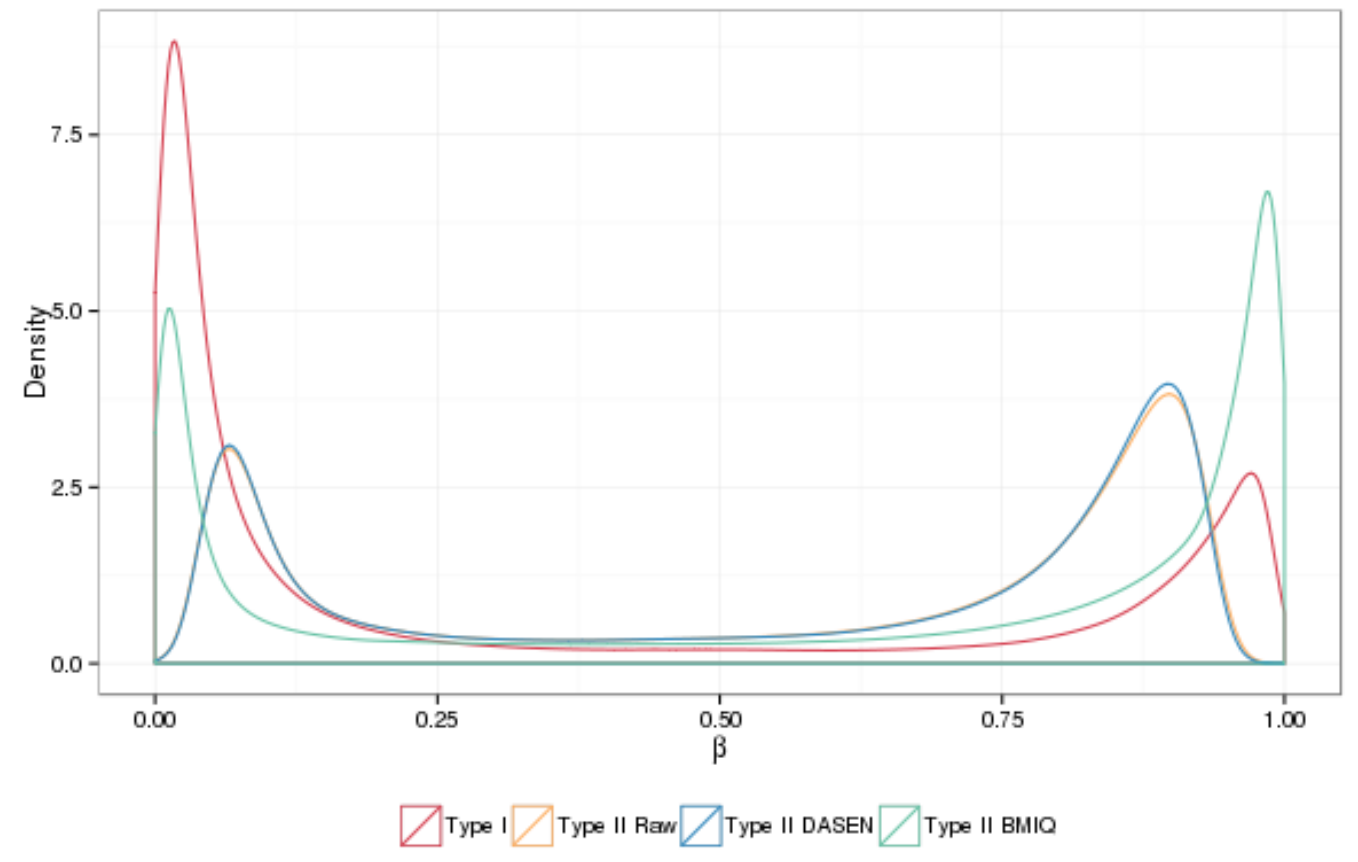


Supplemental Figure 3A: Age analysis t-statistic correlations across all CpG sites.

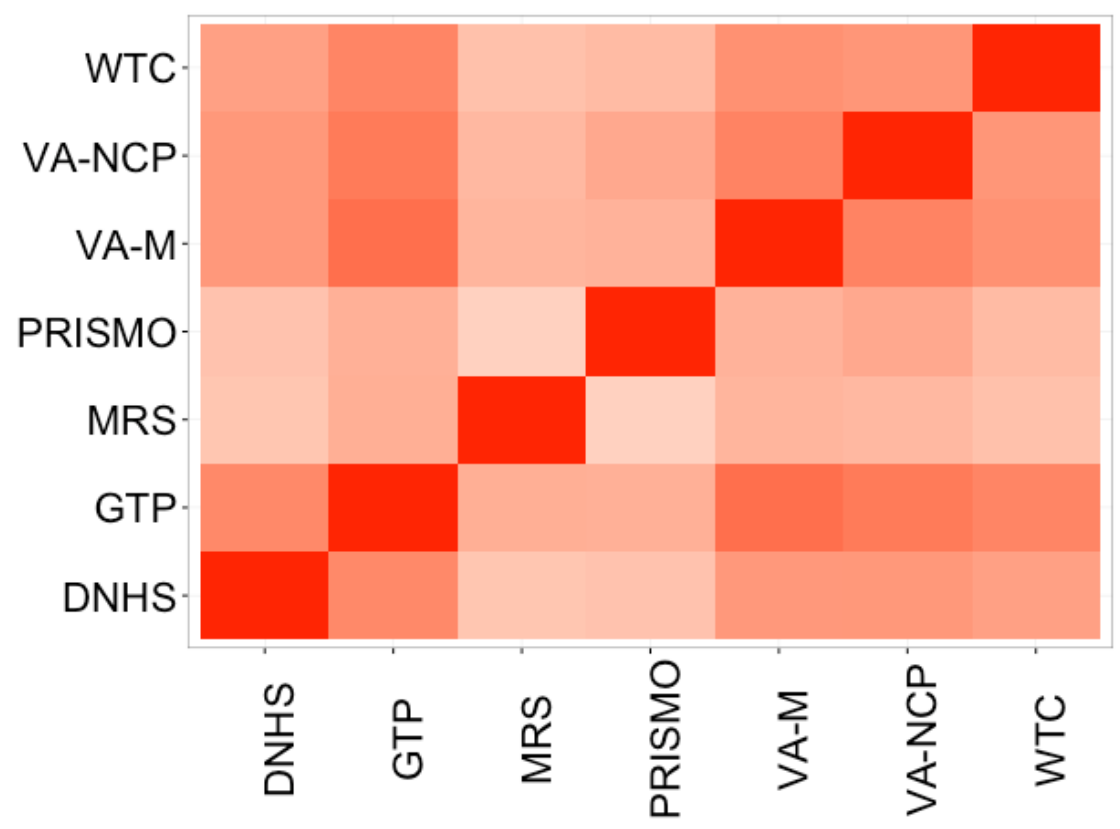

Supplemental Figure 3B: Age analysis t-statistic correlations for the FDR Significant Sites.

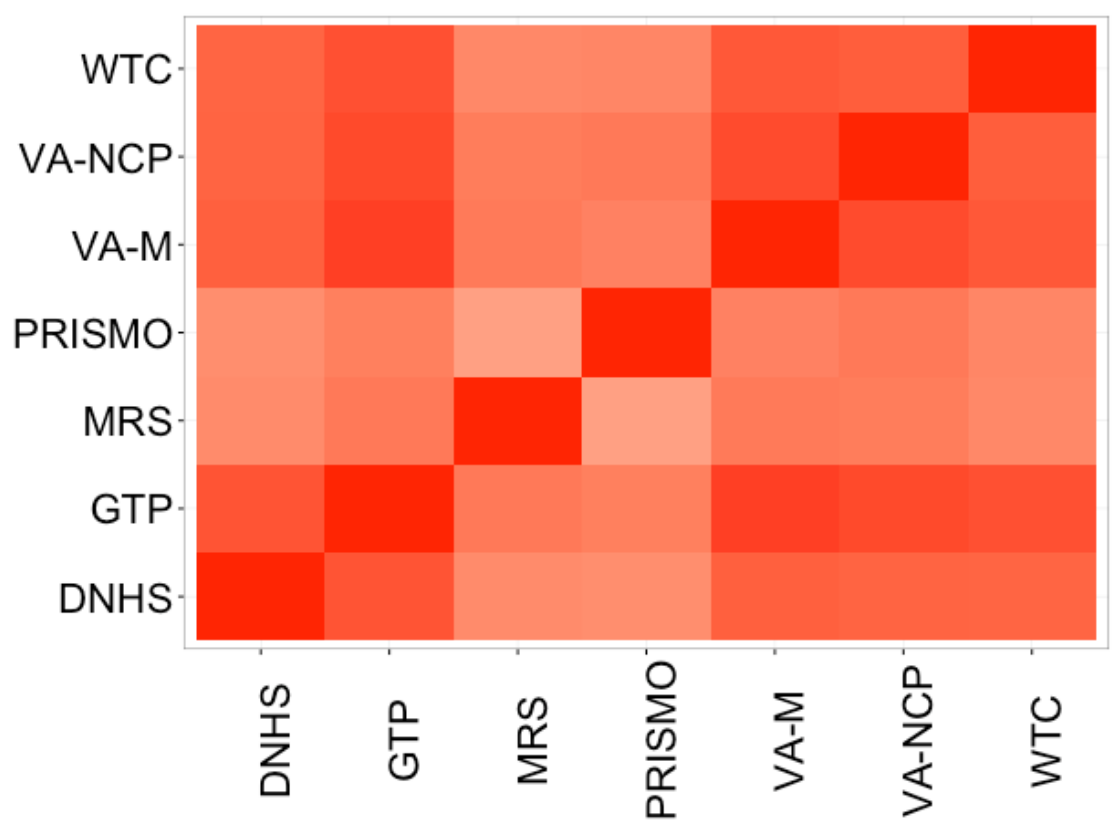


Supplemental Figure 4: Forest Plot of the top CpG site from the analysis of age. Forest plots for significant sites show common direction of effect

$\begin{array}{lll}\text { Study } & \text { Beta } \\ \text { DNHS } & 0.006 \\ \text { GTP } & 0.015 \\ \text { MRS } & 0.013 \\ \text { VA-NCP } & 0.009 & \\ \text { PRISMO } & 0.002 & \\ \text { VA-M } & 0.011 & \\ \text { WTC } & 0.013 \\ \text { Summary } & \mathbf{0 . 0 1 1}\end{array}$




\section{PGC EWAS Cohorts}

\section{Detroit Neighborhood Health Study}

See reference for details (Uddin et al., 2010). Briefly, participants ( $N=1547$ at the baseline wave) were assessed for PTSD symptoms using the PTSD checklist (PCL-C), a 17-item self-report measure of Diagnostic and Statistical Manual of Mental Disorders (DSM-IV) symptoms, and additional questions about duration, timing, and impairment or disability due to the symptoms (Blanchard, Jones-Alexander, Buckley, \& Forneris, 1996). Participants were initially asked to identify potentially traumatic events (PTEs) that they experienced in the past from a list of 19 events. PTSD symptoms were then assessed by referencing two traumatic events that the respondent may have experienced: one that the participant regarded as the worst and one randomly selected event from the remaining PTEs a respondent may have experienced. Respondents were considered affected by lifetime PTSD if all six DSM-IV criteria were met in reference to either the worst or the random event. Current PTSD is defined as meeting the criteria for lifetime PTSD, with symptoms reported during the past month. The DNHS was approved by the institutional review board at the University of Michigan and University of North Carolina at Chapel Hill.

\section{Grady Trauma Project}

See reference for details (Binder et al., 2008). The modified PTSD Symptom Scale (PSS), a psychometrically valid 17-item self-report scale assessing PTSD symptomatology over the prior 2 weeks, was used to assess PTSD. Consistent with prior literature, the PSS frequency items (0 indicates not at all to 3 indicates $\geq 5$ times a week) to obtain a continuous measure of PTSD symptom severity ranging from 0 to 51 . For this sample, the PSS frequency items had standardized $\alpha=.90$ (mean [SD], 13.81 [11.96]). No clearly established PSS cutoff score for PTSD diagnosis has been established; however, DSM-IV criteria for PTSD can be applied to PSS frequency items to create a proxy variable for PTSD diagnostic status. Demographic variables including age, sex and race were assessed through self-report. The Institutional Review Boards of Emory University School of Medicine and Grady Memorial Hospital approved this study.

\section{Marine Resiliency Study}

See reference for details (Baker et al., 2012; C M Nievergelt et al., 2015). PTSD was diagnosed up to 3 times, once before deployment and 3 and/or 6-month post-deployment. Post-traumatic stress (PTS) symptoms were assessed using a structured diagnostic interview, the Clinician Administered PTSD Scale (CAPS), and PTSD diagnosis followed the DSM-IV criteria for partial and full PTSD. The study was approved by the University of California - San Diego Institutional Review Board. 
Mid-Atlantic Mental Illness Research Education and Clinical Center PTSD Study

See reference for details (Ashley-Koch et al., 2015). PTSD was diagnosed using the Structured Clinical Interview for DSM-IV Disorders (SCID) administered by trained interviewers. In accordance with the DSM-IV, PTSD consists of three symptom clusters. These include reexperiencing symptoms (B symptoms), avoidance and numbing symptoms (C symptoms) and hyperarousal symptoms ( $D$ symptoms). Total PTSD symptoms and symptom clusters (,$C$, or $D)$ were measured using the Davidson Trauma Scale for all veterans including individuals with current PTSD diagnosis and controls. The research was reviewed and approved by the Institutional Review Boards at the Salisbury VA, Hampton VA, Durham VA and Duke University Medical Centers.

\section{National Center for PTSD}

See reference for details (Logue et al., 2013). VA study participants were administered the CAPS, a 30-item structured diagnostic interview that assesses the frequency and severity of the 17 DSMIV PTSD symptoms, 5 associated features and functional impairment, to assess current and lifetime PTSD symptoms. The Institutional Review Boards at two VA health care facilities approved the study.

\section{$\underline{\text { PRISMO }}$}

All subjects in the DD were male participants in PRISMO, a large prospective study of 1,032 wellcharacterized Dutch military soldiers scheduled for a deployment of at least four months to Afghanistan with longitudinal follow-up. Baseline measures were recorded at one month predeployment. Follow-up was performed at one month and six months post-deployment, and data from the baseline and six-month follow-up were used for this analysis. A subset (total $\mathrm{N}=93$ ) of three similarly sized subgroups of PRISMO study participants were pre-selected based on the level of traumatic stress exposure and the presence of PTSD symptoms: i) a subgroup showing high combat-trauma exposure $(7.3 \pm 2.9)$ and high levels of post-deployment PTSD symptoms $(45.3 \pm 8.6)$; ii) a subgroup showing high combat-trauma exposure (8.6 \pm 2.3$)$ and a low severity of PTSD symptoms $(26.0 \pm 3.7)$; and iii) a subgroup showing low combat-trauma exposure $(0.4 \pm$ $0.5)$ and low levels of post-deployment PTSD symptoms (25.1 \pm 3.7$)$.

Blood samples were collected six months after deployment. The blood cell-type composition was investigated using flow cytometry, implemented in the clinical laboratory of Utrecht University Medical Center, as previously reported (M P Boks et al., 2015). The presence and severity of symptoms of PTSD over the previous four weeks were assessed with the 22-item Self-Report 
Inventory for PTSD (SRIP), which has good reliability and validity. Differences in PTSD symptoms between time points were log-transformed to improve the distribution. Exposure to traumatic stress during deployment was assessed with a 19-item deployment experiences checklist, as previously reported (van Zuiden et al., 2011).

\section{World Trade Center 9/11 First Responders}

See reference for details (Herbert et al., 2006). First responders were administered the SCID PTSD module with interval instructions (i.e., worst episode of symptoms since 9/11/2001) and SCID items were modified to assess PTSD symptoms in relation to traumatic World Trade Center exposures. The Committees on Research Involving Human Subjects at Stony Brook University approved the study. 
Nagy Youssef's contributions (added for the purpose of this thesis): Nagy Youssef was one of the fellow investigators at the Mid-Atlantic MIRECC when he was at Duke University and Durham VA. He interviewed research patients, supervised SCID and PCL administration, collected the data with other MIRECC investigators, supervised research assistants, and reviewed and edited this paper. 


\section{REFERENCES}

Almli, L. M., Srivastava, A., Fani, N., Kerley, K., Mercer, K. B., Feng, H., ... Ressler, K. J. (2014). Follow-up and extension of a prior genome-wide association study of posttraumatic stress disorder: gene $x$ environment associations and structural magnetic resonance imaging in a highly traumatized African-American civilian population. Biol Psychiatry, 76(4), e3-e4. doi:10.1016/j.biopsych.2014.01.017

American Psychiatric Association, Task Force on DSM-IV. (2000). Diagnostic and statistical manual of mental disorders: DSM-VI-TR. Washington, DC: American Psychiatric Association.

Aryee, M. J., Jaffe, A. E., Corrada-Bravo, H., Ladd-Acosta, C., Feinberg, A. P., Hansen, K. D., \& Irizarry, R. A. (2014). Minfi: a flexible and comprehensive Bioconductor package for the analysis of Infinium DNA methylation microarrays. Bioinformatics, 30(10), 1363-1369. doi:10.1093/bioinformatics/btu049

Ashley-Koch, A. E., Garrett, M. E., Gibson, J., Liu, Y., Dennis, M. F., Kimbrel, N. A., . . Hauser, M. A. (2015). Genome-wide association study of posttraumatic stress disorder in a cohort of Iraq-Afghanistan era veterans. J Affect Disord, 184, 225-234. doi:10.1016/j.jad.2015.03.049

Baker, D. G., Nash, W. P., Litz, B. T., Geyer, M. A., Risbrough, V. B., Nievergelt, C. M., . . WebbMurphy, J. A. (2012). Predictors of risk and resilience for posttraumatic stress disorder among ground combat Marines: methods of the Marine Resiliency Study. Prev Chronic Dis, 9, E97. doi:10.5888/pcd9.110134

Barfield, R. T., Almli, L. M., Kilaru, V., Smith, A. K., Mercer, K. B., Duncan, R., ... Conneely, K. N. (2014). Accounting for population stratification in DNA methylation studies. Genet Epidemiol, 38(3), 231-241. doi:10.1002/gepi.21789

Benjamini, Y., \& Hochberg, Y. (1995). Controlling the false discovery rate: a practical and powerful approach to multiple testing. Journal of the Royal Statistical Society. Series $B$ (Methodological), 57(1), 289-300. doi:10.1111/j.2517-6161.1995.tb02031.x

Benjet, C., Bromet, E., Karam, E. G., Kessler, R. C., McLaughlin, K. A., Ruscio, A. M., . . Koenen, K. C. (2016). The epidemiology of traumatic event exposure worldwide: results from the World Mental Health Survey Consortium. Psychol Med, 46(2), 327-343. doi:10.1017/s0033291715001981

Bernstein, B. E., Stamatoyannopoulos, J. A., Costello, J. F., Ren, B., Milosavljevic, A., Meissner, A., ... Thomson, J. A. (2010). The NIH Roadmap Epigenomics Mapping Consortium. Nat Biotechnol, 28(10), 1045-1048. doi:10.1038/nbt1010-1045

Binder, E. B., Bradley, R. G., Liu, W., Epstein, M. P., Deveau, T. C., Mercer, K. B., . . Nemeroff, C. B. (2008). Association of FKBP5 polymorphisms and childhood abuse with risk of posttraumatic stress disorder symptoms in adults. Jama, 299(11), 1291-1305. doi:10.1001/jama.299.11.1291 
Blanchard, E. B., Jones-Alexander, J., Buckley, T. C., \& Forneris, C. A. (1996). Psychometric properties of the PTSD Checklist (PCL). Behaviour research and therapy, 34(8), 669-673. doi:10.1016/0005-7967(96)00033-2

Bocklandt, S., Lin, W., Sehl, M. E., Sánchez, F. J., Sinsheimer, J. S., Horvath, S., \& Vilain, E. (2011). Epigenetic predictor of age. PloS one, 6(6), 1-6. doi:10.1371/journal.pone.0014821

Boks, M. P., Rutten, B. P., Geuze, E., Houtepen, L. C., Vermetten, E., Kaminsky, Z., \& Vinkers, C. H. (2016). SKA2 methylation is involved in cortisol stress reactivity and predicts the development of post-traumatic stress disorder (PTSD) after military deployment. Neuropsychopharmacology, 41(5), 1350-1356. doi:10.1038/npp.2015.286

Boks, M. P., van Mierlo, H. C., Rutten, B. P., Radstake, T. R., De Witte, L., Geuze, E., . . Broen, J. C. (2015). Longitudinal changes of telomere length and epigenetic age related to traumatic stress and post-traumatic stress disorder. Psychoneuroendocrinology, 51, 506512. doi:0.1016/j.psyneuen.2014.07.011

Bonasio, R., Tu, S., \& Reinberg, D. (2010). Molecular signals of epigenetic states. Science, 330(6004), 612-616. doi:10.1126/science.1191078

Boscarino, J. A. (2008). A prospective study of PTSD and early-age heart disease mortality among Vietnam veterans: implications for surveillance and prevention. Psychosom Med, 70(6), 668-676. doi:10.1097/PSY.0b013e31817bccaf

Brady, K. T., Killeen, T. K., Brewerton, T., \& Lucerini, S. (2000). Comorbidity of psychiatric disorders and posttraumatic stress disorder. Journal of Clinical Psychiatry, 61(suppl 7), 22-32.

Breslau, N., Kessler, R. C., Chilcoat, H. D., Schultz, L. R., Davis, G. C., \& Andreski, P. (1998). Trauma and posttraumatic stress disorder in the community: the 1996 Detroit Area Survey of Trauma. Archives of General Psychiatry, 55(7), 626-632. doi:10.1001/archpsyc.55.7.626

Chang, S.-C., Koenen, K. C., Galea, S., Aiello, A. E., Soliven, R., Wildman, D. E., \& Uddin, M. (2012). Molecular variation at the SLC6A3 locus predicts lifetime risk of PTSD in the Detroit Neighborhood Health Study. PloS one, 7(6), e39184. doi:10.1371/journal.pone.0039184

Coughlin, S. S. (2011). Post-traumatic stress disorder and cardiovascular disease. Open Cardiovasc Med J, 5, 164-170. doi:10.2174/1874192401105010164

Daskalakis, N. P., Cohen, H., Cai, G., Buxbaum, J. D., \& Yehuda, R. (2014). Expression profiling associates blood and brain glucocorticoid receptor signaling with trauma-related individual differences in both sexes. Proc Natl Acad Sci U S A, 111(37), 13529-13534. doi:10.1073/pnas.1401660111

David, D., Woodward, C., Esquenazi, J., \& Mellman, T. A. (2014). Comparison of comorbid physical illnesses among veterans with PTSD and veterans with alcohol dependence. Psychiatric Services, 55(1), 82-85. doi:10.1176/appi.ps.55.1.82

Du, P., Zhang, X., Huang, C.-C., Jafari, N., Kibbe, W. A., Hou, L., \& Lin, S. M. (2010). Comparison of Beta-value and $M$-value methods for quantifying methylation levels by microarray analysis. BMC bioinformatics, 11(1), 587. doi:10.1186/1471-2105-11-587 
Falush, D., Stephens, M., \& Pritchard, J. K. (2003). Inference of population structure using multilocus genotype data: linked loci and correlated allele frequencies. Genetics, 164(4), 1567-1587.

Feinberg, A. P. (2007). Phenotypic plasticity and the epigenetics of human disease. Nature, 447(7143), 433-440. doi:10.1038/nature05919

Fortin, J.-P., Labbe, A., Lemire, M., Zanke, B. W., Hudson, T. J., Fertig, E. J., . . Hansen, K. D. (2014). Functional normalization of 450k methylation array data improves replication in large cancer studies. Genome Biol, 15(12), 503. doi:10.1186/s13059-014-0503-2

Gibbs, J. R., van der Brug, M. P., Hernandez, D. G., Traynor, B. J., Nalls, M. A., Lai, S.-L., . . . Troncoso, J. (2010). Abundant quantitative trait loci exist for DNA methylation and gene expression in human brain. PLoS Genet, 6(5), e1000952. doi:10.1371/journal.pgen.1000952

Guffanti, G., Galea, S., Yan, L., Roberts, A. L., Solovieff, N., Aiello, A. E., . . Koenen, K. C. (2013). Genome-wide association study implicates a novel RNA gene, the lincRNA AC068718.1, as a risk factor for post-traumatic stress disorder in women. Psychoneuroendocrinology, 38(12), 3029-3038. doi:10.1016/j.psyneuen.2013.08.014

Guintivano, J., Brown, T., Newcomer, A., Jones, M., Cox, O., Maher, B. S., . . Kaminsky, Z. A. (2014). Identification and replication of a combined epigenetic and genetic biomarker predicting suicide and suicidal behaviors. American journal of psychiatry, 171(12), 1287 1296. doi:10.1176/appi.ajp.2014.14010008

Hannum, G., Guinney, J., Zhao, L., Zhang, L., Hughes, G., Sadda, S., . . Gao, Y. (2013). Genomewide methylation profiles reveal quantitative views of human aging rates. Molecular cell, 49(2), 359-367. doi:10.1016/j.molcel.2012.10.016

Harper, K. N., Peters, B. A., \& Gamble, M. V. (2013). Batch effects and pathway analysis: two potential perils in cancer studies involving DNA methylation array analysis. Cancer Epidemiology, Biomarkers and Prevention, 22(6), 1052-1060. doi:10.1158/1055-9965.EPI13-0114

Heppner, P. S., Crawford, E. F., Haji, U. A., Afari, N., Hauger, R. L., Dashevsky, B. A., . . Baker, D. G. (2009). The association of posttraumatic stress disorder and metabolic syndrome: a study of increased health risk in veterans. BMC Med, 7, 1. doi:10.1186/1741-7015-7-1

Herbert, R., Moline, J., Skloot, G., Metzger, K., Baron, S., Luft, B., . . . Stein, D. (2006). The World Trade Center disaster and the health of workers: five-year assessment of a unique medical screening program. Environmental health perspectives, 114(12), 1853-1858. doi:10.1289/ehp.9592

Heyn, H., Moran, S., Hernando-Herraez, I., Sayols, S., Gomez, A., Sandoval, J., . . Wang, L. (2013). DNA methylation contributes to natural human variation. Genome research, 23(9), 13631372. doi:10.1101/gr.154187.112

Horvath, S. (2013). DNA methylation age of human tissues and cell types. Genome biology, 14(10), R115. doi:10.1186/gb-2013-14-10-r115 
Horvath, S., Zhang, Y., Langfelder, P., Kahn, R. S., Boks, M., van Eijk, K., . . Ophoff, R. A. (2012). Aging effects on DNA methylation modules in human brain and blood tissue. Genome Biol, 13(10), R97. doi:10.1186/gb-2012-13-10-r97

Houseman, E. A., Accomando, W. P., Koestler, D. C., Christensen, B. C., Marsit, C. J., Nelson, H. H., ... Kelsey, K. T. (2012). DNA methylation arrays as surrogate measures of cell mixture distribution. BMC bioinformatics, 13, 86. doi:10.1186/1471-2105-13-86

Jaffe, A. E., \& Irizarry, R. A. (2014). Accounting for cellular heterogeneity is critical in epigenomewide association studies. Genome Biol, 15(2), R31. doi:10.1186/gb-2014-15-2-r31

Jakovljevic, M., Babic, D., Crncevic, Z., Martinac, M., Maslov, B., \& Topic, R. (2008). Metabolic syndrome and depression in war veterans with post-traumatic stress disorder. Psychiatr Danub, 20(3), 406-410. Retrieved from http://www.ncbi.nlm.nih.gov/pubmed/18827772

Jirtle, R. L., \& Skinner, M. K. (2007). Environmental epigenomics and disease susceptibility. Nature Reviews: Genetics, 8(4), 253-262. doi:10.1038/nrg2045

Johnson, W. E., Li, C., \& Rabinovic, A. (2007). Adjusting batch effects in microarray expression data using empirical Bayes methods. Biostatistics, 8(1), 118-127. doi:10.1093/biostatistics/kxj037

Kessler, R. C. (2000). Posttraumatic stress disorder: the burden to the individual and to society. Journal of Clinical Psychiatry, 61(suppl 5), 4-12.

Kessler, R. C., Berglund, P., Demler, O., Jin, R., Merikangas, K. R., \& Walters, E. E. (2005). Lifetime prevalence and age-of-onset distributions of DSM-IV disorders in the National Comorbidity Survey Replication. Archives of General Psychiatry, 62(6), 593-602. doi:10.1001/archpsyc.62.6.593

Koenen, K. C., Uddin, M., Chang, S. C., Aiello, A. E., Wildman, D. E., Goldmann, E., \& Galea, S. (2011). SLC6A4 methylation modifies the effect of the number of traumatic events on risk for posttraumatic stress disorder. Depression and anxiety, 28(8), 639-647. doi:10.1002/da.20825

Kubzansky, L. D., Koenen, K. C., Jones, C., \& Eaton, W. W. (2009). A prospective study of posttraumatic stress disorder symptoms and coronary heart disease in women. Health Psychol, 28(1), 125-130. doi:10.1037/0278-6133.28.1.125

Kubzansky, L. D., Koenen, K. C., Spiro, A., 3rd, Vokonas, P. S., \& Sparrow, D. (2007). Prospective study of posttraumatic stress disorder symptoms and coronary heart disease in the Normative Aging Study. Arch Gen Psychiatry, 64(1), 109-116. doi:10.1001/archpsyc.64.1.109

Kundaje, A., Meuleman, W., Ernst, J., Bilenky, M., Yen, A., Heravi-Moussavi, A., . . . Kellis, M. (2015). Integrative analysis of 111 reference human epigenomes. Nature, 518(7539), 317330. doi:10.1038/nature14248

Li, J. Z., Absher, D. M., Tang, H., Southwick, A. M., Casto, A. M., Ramachandran, S., . . Myers, R. M. (2008). Worldwide human relationships inferred from genome-wide patterns of variation. Science, 319(5866), 1100-1104. doi:10.1126/science.1153717 
Lin, D. Y., \& Zeng, D. (2010). On the relative efficiency of using summary statistics versus individual-level data in meta-analysis. Biometrika, 97(2), 321-332. doi:10.1093/biomet/asq006

Liu, P., \& Hwang, J. G. (2007). Quick calculation for sample size while controlling false discovery rate with application to microarray analysis. Bioinformatics, 23(6), 739-746. doi:10.1093/bioinformatics/bt|664

Logue, M. W., Amstadter, A. B., Baker, D. G., Duncan, L., Koenen, K. C., Liberzon, I., ... Ressler, K. J. (2015). The Psychiatric Genomics Consortium Posttraumatic Stress Disorder Workgroup: posttraumatic stress disorder enters the age of large-scale genomic collaboration. Neuropsychopharmacology, 40(10), 2287-2297. doi:10.1038/npp.2015.118

Logue, M. W., Baldwin, C., Guffanti, G., Melista, E., Wolf, E. J., Reardon, A. F., .. Koenen, K. C. (2013). A genome-wide association study of post-traumatic stress disorder identifies the retinoid-related orphan receptor alpha (RORA) gene as a significant risk locus. Molecular psychiatry, 18(8), 937-942. doi:10.1038/mp.2012.113

Long, J. S., \& Ervin, L. H. (2000). Using heteroscedasticity consistent standard errors in the linear regression model. The American Statistician, 54(3), 217-224. doi:10.1080/00031305.2000.10474549

Marot, G., Foulley, J.-L., Mayer, C.-D., \& Jaffrézic, F. (2009). Moderated effect size and P-value combinations for microarray meta-analyses. Bioinformatics, 25(20), 2692-2699. doi:10.1093/bioinformatics/btp444

Mathew, T., \& Nordstrom, K. (1999). On the equivalence of meta-analysis using literature and using individual patient data. Biometrics, 55(4), 1221-1223. doi:10.1111/j.0006341X.1999.01221.x

Maunakea, A. K., Nagarajan, R. P., Bilenky, M., Ballinger, T. J., D'Souza, C., Fouse, S. D., . . Zhao, Y. (2010). Conserved role of intragenic DNA methylation in regulating alternative promoters. Nature, 466(7303), 253-257. doi:10.1038/nature09165

McGowan, P. O., Sasaki, A., D'Alessio, A. C., Dymov, S., Labonté, B., Szyf, M., . . Meaney, M. J. (2009). Epigenetic regulation of the glucocorticoid receptor in human brain associates with childhood abuse. Nature Neuroscience, 12(3), 342-348. doi:10.1038/nn.2270

Mehta, D., Klengel, T., Conneely, K. N., Smith, A. K., Altmann, A., Pace, T. W., . . Mercer, K. B. (2013). Childhood maltreatment is associated with distinct genomic and epigenetic profiles in posttraumatic stress disorder. Proc Natl Acad Sci U S A, 110(20), 8302-8307. doi:10.1073/pnas.1217750110

Moser, D. A., Paoloni-Giacobino, A., Stenz, L., Adouan, W., Manini, A., Suardi, F., . . RusconiSerpa, S. (2015). BDNF methylation and maternal brain activity in a violence-related sample. PloS one, 10(12), e0143427. doi:10.1371/journal.pone.0143427 
Najt, P., Fusar-Poli, P., \& Brambilla, P. (2011). Co-occurring mental and substance abuse disorders: a review on the potential predictors and clinical outcomes. Psychiatry Research, 186(2), 159-164. doi:10.1016/j.psychres.2010.07.042

Nielsen, D. A., Hamon, S., Yuferov, V., Jackson, C., Ho, A., Ott, J., \& Kreek, M. J. (2010). Ethnic diversity of DNA methylation in the OPRM1 promoter region in lymphocytes of heroin addicts. Hum Genet, 127(6), 639-649. doi:10.1007/s00439-010-0807-6

Nievergelt, C. M., Maihofer, A. X., Mustapic, M., Yurgil, K. A., Schork, N. J., Miller, M. W., . . . O'Connor, D. T. (2015). Genomic predictors of combat stress vulnerability and resilience in US Marines: a genome-wide association study across multiple ancestries implicates PRTFDC1 as a potential PTSD gene. Psychoneuroendocrinology, 51, 459-471. doi:10.1016/j.psyneuen.2014.10.017

Nievergelt, C. M., Maihofer, A. X., Shekhtman, T., Libiger, O., Wang, X., Kidd, K. K., \& Kidd, J. R. (2013). Inference of human continental origin and admixture proportions using a highly discriminative ancestry informative 41-SNP panel. Investig Genet, 4(1), 13. doi:10.1186/2041-2223-4-13

Olkin, I., \& Sampson, A. (1998). Comparison of meta-analysis versus analysis of variance of individual patient data. Biometrics, 54(1), 317-322. Retrieved from http://www.ncbi.nlm.nih.gov/pubmed/9544524

Orr, M., \& Liu, P. (2009). Sample size estimation while controlling false discovery rate for microarray experiments using the ssize. fdr package. The $R$ Journal, 1, 47-53. doi:10.32614/RJ-2009-019

Pidsley, R., Wong, C. C., Volta, M., Lunnon, K., Mill, J., \& Schalkwyk, L. C. (2013). A data-driven approach to preprocessing Illumina 450K methylation array data. BMC genomics, 14(1), 293. doi:10.1186/1471-2164-14-293

Price, A. L., Patterson, N. J., Plenge, R. M., Weinblatt, M. E., Shadick, N. A., \& Reich, D. (2006). Principal components analysis corrects for stratification in genome-wide association studies. Nat Genet, 38(8), 904-909. doi:10.1038/ng1847

Reinius, L. E., Acevedo, N., Joerink, M., Pershagen, G., Dahlén, S.-E., Greco, D., . . Kere, J. (2012). Differential DNA methylation in purified human blood cells: implications for cell lineage and studies on disease susceptibility. PloS one, 7(7), e41361. doi:10.1371/journal.pone.0041361

Resnick, H. S., Kilpatrick, D. G., Dansky, B. S., Saunders, B. E., \& Best, C. L. (1993). Prevalence of civilian trauma and posttraumatic stress disorder in a representative national sample of women. Journal of consulting and clinical psychology, 61(6), 984-991. doi:10.1037/0022006X.61.6.984

Ressler, K. J., Mercer, K. B., Bradley, B., Jovanovic, T., Mahan, A., Kerley, K., . . May, V. (2011). Post-traumatic stress disorder is associated with PACAP and the PAC1 receptor. Nature, 470(7335), 492-497. doi:10.1038/nature09856 
Rice, L., Waters, C. E., Eccles, J., Garside, H., Sommer, P., Kay, P., . . . Stratford, I. (2008). Identification and functional analysis of SKA2 interaction with the glucocorticoid receptor. Journal of Endocrinology, 198(3), 499-509. doi:10.1677/JOE-08-0019

Roth, T. L., Lubin, F. D., Funk, A. J., \& Sweatt, J. D. (2009). Lasting epigenetic influence of early-life adversity on the BDNF gene. Biol Psychiatry, 65(9), 760-769. doi:10.1016/j.biopsych.2008.11.028

Sareen, J., Houlahan, T., Cox, B. J., \& Asmundson, G. J. (2005). Anxiety disorders associated with suicidal ideation and suicide attempts in the National Comorbidity Survey. J Nerv Ment Dis, 193(7), 450-454. doi:10.1097/01.nmd.0000168263.89652.6b

Sartor, C. E., Grant, J. D., Lynskey, M. T., McCutcheon, V. V., Waldron, M., Statham, D. J., . . . Nelson, E. C. (2012). Common heritable contributions to low-risk trauma, high-risk trauma, posttraumatic stress disorder, and major depression. Archives of General Psychiatry, 69(3), 293-299. doi:10.1001/archgenpsychiatry.2011.1385

Sartor, C. E., McCutcheon, V. V., Pommer, N. E., Nelson, E. C., Grant, J. D., Duncan, A. E., ... Heath, A. C. (2011). Common genetic and environmental contributions to post-traumatic stress disorder and alcohol dependence in young women. Psychol Med, 41(7), 1497-1505. doi:10.1017/S0033291710002072

Sipahi, L., Wildman, D. E., Aiello, A. E., Koenen, K. C., Galea, S., Abbas, A., \& Uddin, M. (2014). Longitudinal epigenetic variation of DNA methyltransferase genes is associated with vulnerability to post-traumatic stress disorder. Psychol Med, 44(15), 3165-3179. doi:10.1017/S0033291714000968

Smith, A. K., Conneely, K. N., Kilaru, V., Mercer, K. B., Weiss, T. E., Bradley, B., . . Ressler, K. J. (2011). Differential immune system DNA methylation and cytokine regulation in posttraumatic stress disorder. American Journal of Medical Genetics Part B: Neuropsychiatric Genetics, 156(6), 700-708. doi:10.1002/ajmg.b.31212

Smith, A. K., Kilaru, V., Kocak, M., Almli, L. M., Mercer, K. B., Ressler, K. J., . . . Conneely, K. N. (2014). Methylation quantitative trait loci (meQTLs) are consistently detected across ancestry, developmental stage, and tissue type. BMC genomics, 15(1), 145. doi:10.1186/1471-2164-15-145

Smyth, G. K. (2005). Limma: linear models for microarray data. In Bioinformatics and computational biology solutions using $R$ and bioconductor (pp. 397-420). New York: SpringerScience+Business Media.

Stein, M. B., Jang, K. L., Taylor, S., Vernon, P. A., \& Livesley, W. J. (2002). Genetic and environmental influences on trauma exposure and posttraumatic stress disorder symptoms: a twin study. Am J Psychiatry, 159(10), 1675-1681. doi:10.1176/appi.ajp.159.10.1675

Teschendorff, A. E., Marabita, F., Lechner, M., Bartlett, T., Tegner, J., Gomez-Cabrero, D., \& Beck, S. (2013). A beta-mixture quantile normalization method for correcting probe design bias in Illumina Infinium 450 k DNA methylation data. Bioinformatics, 29(2), 189-196. doi:10.1093/bioinformatics/bts680 
Thompson, P. M., Stein, J. L., Medland, S. E., Hibar, D. P., Vasquez, A. A., Renteria, M. E., .. Epigen Consortium Imagen Consortium Saguenay Youth Study Group. (2014). The ENIGMA Consortium: large-scale collaborative analyses of neuroimaging and genetic data. Brain Imaging Behav, 8(2), 153-182. doi:10.1007/s11682-013-9269-5

True, W. R., Rice, J., Eisen, S. A., Heath, A. C., Goldberg, J., Lyons, M. J., \& Nowak, J. (1993). A twin study of genetic and environmental contributions to liability for posttraumatic stress symptoms. Arch Gen Psychiatry, 257-264. doi:10.1001/archpsyc.1993.01820160019002

Tyrka, A. R., Price, L. H., Marsit, C., Walters, O. C., \& Carpenter, L. L. (2012). Childhood adversity and epigenetic modulation of the leukocyte glucocorticoid receptor: preliminary findings in healthy adults. PloS one, 7(1), e30148. doi:10.1371/journal.pone.0030148

Uddin, M., Aiello, A. E., Wildman, D. E., Koenen, K. C., Pawelec, G., de Los Santos, R., ... Galea, S. (2010). Epigenetic and immune function profiles associated with posttraumatic stress disorder. Proc Natl Acad Sci U S A, 107(20), 9470-9475. doi:10.1073/pnas.0910794107

van Zuiden, M., Geuze, E., Willemen, H. L., Vermetten, E., Maas, M., Heijnen, C. J., \& Kavelaars, A. (2011). Pre-existing high glucocorticoid receptor number predicting development of posttraumatic stress symptoms after military deployment. American journal of psychiatry, 168(1), 89-96. doi:10.1176/appi.ajp.2010.10050706

Vinkers, C. H., Kalafateli, A. L., Rutten, B. P., Kas, M. J., Kaminsky, Z., Turner, J. D., \& Boks, M. P. (2015). Traumatic stress and human DNA methylation: a critical review. Epigenomics, 7(4), 593-608. doi:10.2217/epi.15.11

Weaver, I. C. G., Cervoni, N., D'Alessio, A. C., Champagne, F. A., Seckl, J. R., Szyf, M., \& Meaney, M. J. (2004). Epigenetic programming through maternal behavior. Nature Neuroscience, 7, 847-854. doi:10.1038/nn1276

Weidner, C. I., Lin, Q., Koch, C. M., Eisele, L., Beier, F., Ziegler, P., . . Mühleisen, T. W. (2014). Aging of blood can be tracked by DNA methylation changes at just three CpG sites. Genome biology, 15(2), R24. doi:10.1186/gb-2014-15-2-r24

Wisco, B. E., Marx, B. P., Wolf, E. J., Miller, M. W., Southwick, S. M., \& Pietrzak, R. H. (2014). Posttraumatic stress disorder in the US veteran population: results from the National Health and Resilience in Veterans Study. Journal of Clinical Psychiatry, 75(12), 1338-1346. doi:10.4088/JCP.14m09328

Xian, H., Chantarujikapong, S. I., Scherrer, J. F., Eisen, S. A., Lyons, M. J., Goldberg, J., . . . True, W. R. (2000). Genetic and environmental influences on posttraumatic stress disorder, alcohol and drug dependence in twin pairs. Drug \& Alcohol Dependence, 61(1), 95-102. doi:10.1016/S0376-8716(00)00127-7

Xie, P., Kranzler, H. R., Yang, C., Zhao, H., Farrer, L. A., \& Gelernter, J. (2013). Genome-wide association study identifies new susceptibility loci for posttraumatic stress disorder. Biol Psychiatry, 74(9), 656-663. doi:10.1016/j.biopsych.2013.04.013 
Zeileis, A. (2004). Econometric computing with $\mathrm{HC}$ and $\mathrm{HAC}$ covariance matrix estimators. Journal of Statistical Software, 11(1), 1-17. doi:10.18637/jss.v011.i10 


\section{Chapter 5 A 3-Year Longitudinal Study Examining the Effect of Resilience on Suicidality in Veterans}

Nagy A. Youssef, $\mathrm{MD}^{1,2}$; Kimberly T. Green, $\mathrm{MS}^{1,2}$; Jean C. Beckham, $\mathrm{PhD}^{1,2}$; Eric B. Elbogen, $\mathrm{PhD}^{1,3}$

${ }^{1}$ Department of Psychiatry and Behavioral Sciences, Duke University Medical Center.

2 Mid-Atlantic Mental Illness Research, Education, Durham, NC, USA.

${ }^{3}$ Department of Psychiatry, University of North Carolina, Chapel Hill, NC, USA.

Published as: Youssef NA, Green KT, Beckham JC, Elbogen EB. A 3-year longitudinal study examining the effect of resilience on suicidality in veterans. Ann Clin Psychiatry. 2013 Feb;25(1):59-66. PubMed PMID: 23376871; PubMed Central PMCID: PMC3622865.

https://www.aacp.com/article/buy now/?id=132 


\section{Abstract}

Background: This study prospectively evaluated the correlation and role of resilience and resilience factors in predicting suicidal ideation and attempts in veterans.

Methods: In this 3-year longitudinal study, 178 Iraq and Afghanistan War veterans were evaluated for a number of clinical and demographic variables. Longitudinal follow-up was performed at approximately 3 years.

Results: Resilience at the initial assessment predicted lower suicidality at follow-up, controlling for suicidality at the initial assessment, suggesting a protective effect for resilience. With respect to specific domains of resilience, secure relationships and positive acceptance of change significantly predicted lower suicidality.

Conclusions: These findings have important implications for clinical care and for guiding future research efforts to increase resilience among returning soldiers.

\section{Keywords}

Suicidality, psychological resilience, veterans, resilience factors, secure relationships 


\section{INTRODUCTION}

Studies indicate high rates of mental health problems (Erbes, Westermeyer, Engdahl, \& Johnsen, 2007; Friedman, 2006; Hoge, Auchterlonie, \& Milliken, 2006; Jakupcak, Luterek, Hunt, Conybeare, \& McFall, 2008; Milliken, Auchterlonie, \& Hoge, 2007; Zivin et al., 2007) and suicidality (Kaplan, Huguet, McFarland, \& Newsom, 2007) among returning soldiers. As military troops return from Iraq and Afghanistan, addressing post-deployment adjustment problems, including suicidality, is an important priority (Hoge et al., 2006; Hoge et al., 2004; Milliken et al., 2007).

Suicide risk factors in veteran populations have been studied extensively. Studies have indicated that psychiatric disorders, particularly depression and substance use disorders, are strong risk factors for suicide in most cases (American Psychiatric Association, 2003). Other important risk factors include posttraumatic stress disorder (PTSD) (Guerra, Calhoun, \& MidAtlantic Mental IIIness Research, 2011; Panagioti, Gooding, \& Tarrier, 2009), previous suicide attempts, and demographic factors such as age, sex, and ethnicity (American Psychiatric Association, 2003). Additionally, combat trauma (Bryan \& Cukrowicz, 2011) and childhood abuse (Roy, 2011) have been found to be risk factors for suicide. Some of these factors are modifiable, such as reducing or controlling symptoms of psychiatric disorders, while others, such as age, sex, and history of suicide attempts, are not.

Despite extensive literature on understanding and treating risk factors for suicide, little empirical progress has been made toward preventing suicide in military service members, among whom there has been an alarming increase in suicide (Bachynski et al., 2012). In one study, the risk of death from disease in general did not differ between veterans and the nonveterans. However, veterans were twice as likely (adjusted hazard ratio [HR], 2.13; 95\% Cl, 1.14 to 3.99) to die of suicide compared with nonveterans in the general population (Kaplan et al., 2007).

Clinical and research data from other disciplines have indicated that reducing risk factors should be paired with boosting protective factors. For example, if eliminating risk factors for myocardial infarction (e.g., smoking cessation) is augmented with protective factors (e.g., physical exercise), marked reduction in rates of myocardial infarction can be achieved. The same approach could help reduce suicide rates.

However, limited data are available on protective factors against suicide, especially among veterans. One possible protective factor is resilience. Resilience is a construct that has received more attention in recent years. It is defined by Connor and Davidson as "qualities that enable one to thrive in the face of adversity"(Connor \& Davidson, 2003). It also has been measured and defined by the well-validated Connor-Davidson Resilience Scale (CD-RISC) (Connor \& Davidson, 2003; Yu, Lau, Mak, Zhang, \& Lui, 2011) and has been suggested to be multidimensional (Brunet, McDonough, Hadd, Crocker, \& Sabiston, 2010; Leve, Fisher, \& Chamberlain, 2009; O'Donnell, Schwab-Stone, \& Muyeed, 2002; Wright, Fopma-Loy, \& Fischer, 2005). Resilience can improve in patients with PTSD, with significant reduction in CD-RISC scores after pharmacotherapeutic and psychotherapeutic interventions (J. Davidson et al., 2008; J. R. Davidson et al., 2005). 
Studies that have examined resilience and suicidality assessed this relationship crosssectionally. These studies suggested that resilience might be a protective factor against suicide risk (Green, Calhoun, Dennis, \& Beckham, 2010; Lockwood \& Youssef; Nrugham, Holen, \& Sund, 2010; Pietrzak et al., 2010; Roy, Carli, \& Sarchiapone, 2011).

However, assessment of resilience longitudinally is needed to understand this relationship across time. Moreover, there is no study that has examined which factor(s) (of 5 factors of resilience described by the Connor and Davidson factor analysis (Connor \& Davidson, 2003)) is/are correlated with suicide.

Understanding the relationship between resilience and suicide in returning veterans is helpful for many reasons. First, it could help identify individuals who are at the highest risk for suicide, therefore providing further training or excluding these individuals from situations associated with high trauma exposure. Second, interventions that have the most resilienceenhancing effect could be further examined to determine if they could lower the suicide rate. Finally, examining which factor(s) are correlated with resilience might help in psychotherapeutic and psychosocial treatment development to boost resilience and has implications for treatment policy.

This study extends our previous work (Green et al., 2010; Lockwood \& Youssef). Our a priori hypothesis is that across time, higher resilience would predict lower suicidality. Second, we also explored how the 5 individual factors of resilience (Connor \& Davidson, 2003) predicted protection against suicidality across time.

\section{METHODS}

\section{Participants and Procedures}

In this longitudinal study, 176 Operation Enduring Freedom, Operation Iraqi Freedom and Operation New Dawn (OEF/OIF/OND) veterans were interviewed at the Veterans Integrated Service Network (VISN) 6 Mental Illness Research, Education, and Clinical Center (MIRECC). The MIRECC houses a research registry of veterans who served in the US Armed Forces after September 11, 2001, and volunteered to be considered for research. All veterans were separated from active duty or were in the National Guard/Reserve.

Independent variables on veteran risk factors were gathered at baseline interviews, which occurred between 2005 and 2008. This was designated at time 1 (T1). Veterans in the MIRECC registry were recruited through mailings, advertisements, and clinician referrals. Veterans completed informed consent procedures that were approved by VA institutional review boards at multiple sites in North Carolina and Virginia and afterward were administered questions concerning post-deployment adjustment. Veterans were compensated for their time and travel expenses.

Dependent variables on veterans' suicidality were gathered from follow-up interviews, designated as time 2 (T2). An average of 3 years after the baseline interview, veterans in the MIRECC registry were contacted and asked to participate in a National Institute of Mental Health study, which served as the follow-up interview for the current study. Because this study is part 
of a registry database with a 3-year follow-up, the raters did not know the outcome measures or the purpose of this analysis while evaluating participants. Veterans were compensated for their time and travel expenses after the interviews.

\section{Measures}

Beck Scale for Suicide Ideation (BSI): Suicidality was assessed using the 21-item, selfreport BSI (Beck \& Steer, 1991). Severity of suicidal thoughts, intent, and plans are assessed in items 1 to 19 . The number of previous suicide attempts and the seriousness of the attempt to die associated with the last attempt are assessed in items 20 and 21 . Item responses are rated on a 3-point scale ranging from 0 to 2 and then summed to arrive at a total score. The BSI has demonstrated strong internal reliability (reported $\alpha$ coefficient between 0.90 and 0.97 (Beck, Steer, \& Ranieri, 1988; Steer, Rissmiller, Ranieri, \& Beck, 1993)). BSI has reasonable convergent, discriminative and predictive validity (Beck, Brown, \& Steer, 1997; Beck et al., 1988; Brown, Beck, Steer, \& Grisham, 2000; Cochrane-Brink, Lofchy, \& Sakinofsky, 2000). Brown et al. used a cutoff score of 3 to create a dichotomous index of suicidality, using survival analyses. They determined that a cutoff score of 3 on the BSI yielded the highest hazard ratio in prediction of suicide risk. Given these data, as well as the fact that the BSI deviated markedly from normality in our sample, we employed this cutoff score in our current study to create a dichotomous index $(\leq 2$ or $\geq 3)$ of suicidality.

\section{Connor-Davidson Resilience Scale (CD-RISC):}

The CD-RISC is a reliable (Cronbach $\alpha=0.96$ in the current sample) and valid (Connor \& Davidson, 2003) instrument for measuring resilience. The scale was administered and validated in several different populations, including a community sample, primary care outpatients, general psychiatric outpatients, participants with generalized anxiety disorder and participants with PTSD.

Items on the CD-RISC assess resilience and include items such as "can deal with whatever comes," "past success gives confidence for new challenge," "see the humorous side of things," "coping with stress strengthens," "tend to bounce back after illness or hardship," "things happen for a reason," "best effort no matter what," "not easily discouraged by failure," "think of self as strong person," "strong sense of purpose" and "in control of your life."

The scale demonstrated sound psychometric properties (Connor \& Davidson, 2003). The CD-RISC has demonstrated sensitivity to treatment effects with several therapies (including sertraline, paroxetine, venlafaxine, and cognitive behavioral therapy) in PTSD patients over time (J. Davidson et al., 2008; J. R. Davidson et al., 2005). This scale consists of 25 items. Each item is rated on a 5-point Likert scale with a possible score from 0 to 100 . Higher scores reflect greater resilience. This was used to measure global resilience.

Factor analysis as performed by Connor and Davidson yielded 5 factors. These factors were interpreted in the following manner: factor 1 reflects personal competence, high standards, and tenacity; factor 2 relates to trust in one's instincts, tolerance of negative affect, and strengthening effects of stress; factor 3 corresponds to the positive acceptance of change and 
secure relationships; factor 4 relates to control; and factor 5 to the effect of spirituality. The 5 factors' eigenvalues were 7.47, 1.56, 1.38, 1.13, and 1.07, respectively (Connor \& Davidson, 2003). In this study, we examined how the 5 factors of resilience at time 1 predicted suicidality at time 2.

\section{Davidson Trauma Scale (DTS):}

PTSD severity was assessed using the DTS (J. R. Davidson et al., 1997; McDonald, Beckham, Morey, \& Calhoun, 2009). The DTS is a brief global assessment scale for PTSD symptoms. It includes 17 items corresponding to DSM-IV symptoms of PTSD. The 17 items are rated by frequency and severity. Reliability and validity of the DTS has been demonstrated in veterans who have served since September 11, 2001 (McDonald et al., 2009).

\section{Alcohol Use Disorders Identification Test (AUDIT):}

AUDIT is a 10-item, self-report screening questionnaire used to identify individuals with problematic patterns of alcohol consumption. AUDIT is divided into 3 domains: hazardous alcohol use (questions 1 to 3 ), dependence symptoms (questions 4 to 6 ), and harmful alcohol use (questions 7 to 10). Each response has a score ranging from 0 to 4 . A total score of $\geq 8$ in men, or $\geq 7$ in women, indicates a strong likelihood of hazardous and harmful alcohol use, as well as possible alcohol dependence. The AUDIT has been validated for DSM-IV alcohol use disorders.

\section{Analysis procedures}

All statistical analyses were performed using SAS, version 9.2 for Windows (SAS Institute). Univariate analyses were used to describe the sample. Spearman correlation analyses were conducted to ascertain bivariate relationships between T2 suicidality and T1 variables. Analyses by multiple logistic regression were subsequently subjected to stepwise deletion to obtain a reduced model; exclusion criteria were set at a conservative level of $p<0.05$. Specifically, T2 suicidality was regressed on T1 suicidality, T1 resilience, and other clinical and demographic covariates. A parallel analysis was conducted that included multiple domains of resilience derived from previous factor analyses (Connor \& Davidson, 2003).

\section{RESULTS}

Demographic and clinical characteristics are shown in Table 1. Participants $(N=176)$ were mean age of $39(S D=10.6)$ and were primarily white $(63 \%)$. The study sample was largely men $(82 \%)$, and the majority completed post-high school education (57\%).

\section{Resilience factors protective against suicidality}


Table 2 displays Spearman correlation coefficients between suicidality and resilience factors. There was a significant negative correlation between suicidality and secure relationships ( $r s=-0.34 ; p<0.0001)$ and control ( $r s=-0.29 ; P=.0004)$. A trend was found between suicidality and tolerance ( $r s=-0.16 ; P=.049$ ). There was neither significant correlation between suicidality and tenacity, nor between suicidality and spirituality.

We also examined resilience factors (Connor \& Davidson, 2003) most predictive of suicidality across time using multivariate regression. The factor corresponding to secure relationships and positive acceptance of change $(r 2=0.04 ; F=8.19, p=0.005)$ was most predictive both in the bivariate analysis (Table 2 ) and in the multivariate regression (Table 3). Although control (factor 4 of resilience) was correlated with suicidality in the bivariate correlation, it was not correlated when examined in the multivariate model.

\section{Resilience is protective against suicidality in multivariate model}

The results of the multivariate forward stepwise regression are summarized in Table 4. Resilience at baseline (T1) was found to be predictive of suicidality at follow-up (T2) (R2=0.17; $F=3.95 ; p=0.0485$ ), controlling for several demographic and clinical variables (Table 4) including suicidality at baseline $(p<0.0001)$ (Connor \& Davidson, 2003). Low resilience was a stronger predictor of suicidality than alcohol misuse (on the AUDIT) and PTSD.

\section{DISCUSSION}

In this study, suicidality and resilience have been shown to be inversely related across time. Longitudinal follow-up was performed at approximately 3 years. This suggests a protective effect of resilience on suicidality. Of the 5 factors of resilience, the factor corresponding to secure relationships and positive acceptance of change was most significantly predictive of lower suicidality.

To our knowledge, this is the first study to examine the relationship between suicidality and resilience longitudinally. Our result of an inverse relationship between global resilience and suicidality is in agreement with and extends the results of the few cross-sectional studies that have previously examined this relationship (Green et al., 2010; Lockwood \& Youssef; Nrugham et al., 2010; Pietrzak et al., 2010; Roy et al., 2011). One of these cross-sectional studies is by Roy et al., who examined the relationship between suicidality and resilience. Their sample included 20 abstinent substance abuse patients who had attempted suicide, and another sample of 166 prisoners who had attempted suicide and matched control groups (Roy et al., 2011). In these samples, those who had never attempted suicide had significantly higher CD-RISC resilience scores compared with those who had attempted suicide. The authors reported that the study results "suggest that resilience may be a protective factor mitigating the risk of suicidal behavior associated with childhood trauma" (Roy et al., 2011). 
Another study included 272 Iraq and Afghanistan veterans who were assessed crosssectionally with a survey that contained measures of resilience. Thirty-four participants (12.5\%) reported that they were contemplating suicide in the 2 weeks prior to the survey. Suicide contemplators had lower scores of resilience on CD-RISC. The investigators also found that suicide risk associated with childhood trauma was associated with lower resilience. Of relevance to our study, the investigators found that "sense of purpose and control were negatively associated with suicidal ideation" (Pietrzak et al., 2010).

Moreover, a study by Green et al. showed that resilience is inversely related to PTSD in veterans and to functional response to PTSD. In another study by our group, resilience has been shown to mitigate the effect of depression and suicidal ideation in Iraq and Afghanistan war veterans cross-sectionally (Lockwood \& Youssef).

Although a study by Nrugham et al. (Nrugham et al., 2010) examined this relationship in a longitudinal study, resilience was measured cross-sectionally. The study examined a subset sample of 252 participants from eighth and ninth grade. The participants were followed up after 1 year and reassessed 5 years later. Resilience was assessed using the CD-RISC only at the last visit of the study (T3). The investigators found high resilience to be negatively associated with suicide attempts even if the adolescents were victims of violent life events and had been depressed at age 15 .

It should be noted that secure relationships were significantly correlated with lower suicidality in the bivariate and multivariate model. However, in the multivariate model, secure relationships contributed to a small portion of the variance of approximately $13 \%$. It is not clear why that is so and needs to be further validated in future studies. Possible explanations could be moderation due to other variables. Potential interacting and moderating effects that are worth examining in future studies are sex, age, and clinical variables such as PTSD diagnosis (Carter et al., 2011; Youssef et al., 2013).

Several investigators have stated that longitudinal studies are needed to increase the robustness of the relationship between resilience and suicidality, and to examine the relationship across time (Sarchiapone, Carli, Cuomo, \& Roy, 2007). The current study fills this gap and confirms the previous finding, suggesting the protective effect of resilience across time. This is the first study to examine the relationship between suicidality and global resilience longitudinally, and the first to examine the factors of resilience individually across time. Interestingly, our longitudinal study results suggest that low resilience was a stronger predictor of suicidality than alcohol misuse and PTSD.

Increasing the confidence that this relationship between resilience and suicidality still holds across time paves the way for development of further programs and interventions to address and boost resilience as an important target for reducing suicidality above and beyond reducing risk factors for suicidality. 
These interventions would best address psychosocial aspects that can bolster resilience, improve acceptance to change, and improve relationships. In addition, initial clinical trials of psychopharmacological agents have provided early evidence that resilience can be enhanced pharmacologically (J. Davidson et al., 2008; J. R. Davidson et al., 2005). In addition, an exploratory study has found pretreatment resilience to be a predictor of positive treatment response to the antidepressant venlafaxine (J. R. Davidson et al., 2012). Therefore, further examination of this line of research might lead to important interventional modalities for both preventive and treatment purposes above and beyond symptom control.

However, these results should be interpreted with caution in light of the study's limitations. For example, the possibility of other confounding effects could not be eliminated because of the observational design of the study. Potential confounders such as schizophrenia or bipolar disorder diagnoses also are limitations of this observational study. However, these disorders generally have a low base rate among military veterans because they are screened out during military admission evaluations. Another limitation of the present study is that it included a convenience sample of those who completed the interviews and rating scales. Baseline assessment consisted of self-selected veterans. As a result, participants never were initially approached at baseline. With respect to the follow-up, about one-quarter of initial patients had inaccurate contact information and could not be followed. Another limitation is that suicidality (suicidal ideation and attempts) - not completed suicide-was examined in this study. However, assessment of suicidality is the standard of clinical care and a proxy for assessing the risk of suicide (American Psychiatric Association, 2003). Also, a potential limitation is confounding due to psychotropic medications because of the FDA warning of suicidality for antidepressants and anticonvulsants. However, the contribution of antidepressants and anticonvulsants to suicidality (beyond the suicidality due to the primary mental illness) recently has been called into question from multiple rigorous studies (Gibbons, Brown, Hur, Davis, \& Mann, 2012; Gibbons, Hur, Brown, \& Mann, 2009, 2010; Isacsson, Holmgren, Osby, \& Ahlner, 2009; Isacsson, Reutfors, Papadopoulos, Osby, \& AhIner, 2010; Isacsson, Rich, Jureidini, \& Raven, 2010; Oquendo et al., 2011).

Despite these limitations, initial results from this longitudinal observational study are clinically informative and encouraging and would inform further studies that replicated this work. These results suggest that comprehensive assessment of resilience and its factors among veterans can contribute to understanding their clinical status in terms of suicidality and can inform clinical care. These may be important steps toward development of targeted preventive therapeutics programs, especially toward suicide prevention. In addition, the results could help direct future programs and therapeutics to bolster resilience and reduce suicidal ideation and attempts. Both the Department of Veterans Affairs and the Department of Defense have efforts under way to potentially increase resilience among active duty soldiers (Reivich, Seligman, \& McBride, 2011) and veterans (Bates et al., 2010). 


\section{CONCLUSION}

This is the first longitudinal study to establish the protective effect of resilience in veterans with suicidality. However, further longitudinal studies may increase confidence in these findings if the results are replicated in a larger sample and in other populations. Also, further research in this area with candidate interventions is still needed. 
Acknowledgments: We extend thanks to the veterans who volunteered to participate in this study. We thank the diligent Mid-Atlantic Mental Illness Research, Education and Clinical Center Workgroup members and investigators, whose workgroup for this manuscript includes Mira Brancu, PhD, and John A. Fairbank, PhD, Durham VA Medical Center, Durham, NC; Antony Fernandez, MD, Hunter Holmes McGuire Department of Veterans Affairs Medical Center, Richmond, VA; Harold Kudler, MD, and Christine E. Marx, MD, Durham VA Medical Center, Durham, NC; Scott D. McDonald, PhD, Hunter Holmes McGuire Department of Veterans Affairs Medical Center, Richmond, VA; Scott D. Moore, MD, PhD, and Rajendra A. Morey, MD, Durham VA Medical Center, Durham, NC; Treven C. Pickett, PsyD, Hunter Holmes McGuire Department of Veterans Affairs Medical Center, Richmond, VA; Kristy Straits-Troster, PhD, and Jennifer L. Strauss, PhD, Durham VA Medical Center, Durham, NC; Katherine H. Taber, PhD, W. G. (Bill) Hefner VA Medical Center, Salisbury, NC; Larry A. Tupler, PhD, Elizabeth E. Van Voorhees, PhD, and Richard D. Weiner, MD, PhD, Durham VA Medical Center, Durham, NC; and Ruth E. YoashGantz, PsyD, W. G. (Bill) Hefner VA Medical Center, Salisbury, NC. We also thank the research study staff members at the Durham, Hampton, Richmond, and Salisbury Veterans Affairs Medical Centers for their diligent work and essential contributions to the recruitment of participants and data collection and management of this study. We would also like to acknowledge Mr. Perry Whitted and Ms. Misty Brooks for their extensive administrative contributions on this project, as well as Mr. Jeffery Hoerle for his computer expertise devoted to the project. Mr. Whitted, Ms. Brooks, and Mr. Hoerle report no conflict of interest.

Disclosures: This work was supported by the Office of Mental Health Services, Department of Veterans Affairs; VISN 6 Mid-Atlantic MIRECC; and Office of Academic Affiliation (OAA), the Department of Veterans Affairs (NAY), NIMH grant R01MH080988 (EBE). 
Table 1

Demographics and clinical characteristics at baseline

\begin{tabular}{|l|l|}
\hline Variable & $\begin{array}{l}\text { Total sample } \\
(\mathrm{N}=176)\end{array}$ \\
\hline Age, years & Mean (SD) \\
\hline Education post high school (\%) & $57 \%$ \\
\hline Sex, male (\%) & $82 \%$ \\
\hline Race, white (\%) & $63 \%$ \\
\hline $\begin{array}{l}\text { Days between baseline and follow- } \\
\text { up visit }\end{array}$ & $1057(174)$ \\
\hline Suicidality & \\
\hline Baseline BSS & $16 \%$ \\
\hline Follow-up BSS & $0.50(1.9)$ \\
\hline Resilience (CD-RISC) & $0.55(2.3)$ \\
\hline Alcohol use disorder (AUDIT) & $76.7(15.7)$ \\
\hline PTSD (SCID) & $8 \%$ \\
\hline
\end{tabular}

AUDIT: Alcohol Use Disorders Identification Test; BSS: Beck Scale for Suicide Ideation; CD-RISC, Connor Davidson Resilience Scale; PTSD: posttraumatic stress disorder; SCID: Structured Clinical Interview for DSM IV-Research Version; SD: standard deviation. 
Table 2

Spearman correlation coefficients between suicidality and resilience factors

\begin{tabular}{|l|l|l|}
\hline Variables & $r_{s}$ & $P$ \\
\hline Tenacity (factor 1) & -0.15 & .06 \\
\hline Tolerance (factor 2) & -0.16 & .049 \\
\hline $\begin{array}{l}\text { Secure relationships } \\
\text { (factor 3) }\end{array}$ & -0.34 & $<.0001$ \\
\hline Control (factor 4) & -0.29 & .0004 \\
\hline Spiritual (factor 5) & -0.10 & .22 \\
\hline
\end{tabular}


Table 3

Forward stepwise regression of the total sample for resilience factors on follow-up suicidality ${ }^{a}$

\begin{tabular}{|l|l|l|l|l|l|}
\hline Variable & Partial $R^{2}$ & Model $R^{2}$ & $\mathrm{C}(\mathrm{p})$ & $F$ value & $P$ value \\
\hline & & & & & \\
\hline Baseline BSS secure & 0.09 & 0.90 & 19.93 & 16.73 & $<.0001$ \\
\hline $\begin{array}{l}\text { Baseline } \\
\text { relationships }\end{array}$ & 0.04 & 0.13 & 13.92 & 7.52 & .007 \\
\hline
\end{tabular}

a All variables left in the model are significant at the 0.05 level. No other variable met the 0.05 significance level for entry into the model.

b Secure relationships factor on the Connor Davidson Resilience Scale (factor 3).

BSS: Beck Scale for Suicide Ideation. 


\section{Table 4}

Forward stepwise regression of the total sample for baseline resilience score on follow-up suicidality (BSS) ${ }^{a}$

\begin{tabular}{|l|l|l|l|l|l|}
\hline Variable & Partial $R^{2}$ & Model $R^{2}$ & $\mathrm{C}(\mathrm{p})$ & $F$ value & $P$ value \\
\hline & & & & & \\
\hline Baseline BSS & 0.09 & 0.09 & 19.65 & 16.43 & $<.0001$ \\
\hline Baseline CD-RISC & 0.01 & 0.17 & 8.61 & 4.02 & .047 \\
\hline
\end{tabular}

aAll variables left in the model are significant at the .05 level. No other variable met the .05 significance level for entry into the model.

The other variables included in the model were education, sex, ethnicity, race, PTSD, alcohol misuse on the AUDIT, and major depressive disorder.

These variables (except sex) did not meet the .05 significance level for entry into the final model. Thus, resilience was a stronger predictor than PTSD or alcohol for suicidality.

AUDIT: Alcohol Use Disorders Identification Test; BSS: Beck Scale for Suicide Ideation; CD-RISC: Connor Davidson Resilience Scale; PTSD: posttraumatic stress disorder. 
Nagy Youssef's contributions (added for the purpose of this thesis): Nagy Youssef was one of the fellow investigators at the Mid-Atlantic MIRECC when he was at Duke and Durham VA. He interviewed research patients, supervised SCID and PCL administration, collected the data with other MIRECC investigators, supervised research assistants and reviewed and edited this paper. He also worked with the statistician to guide the statistical analysis of this paper along with the other coauthors. He wrote the first draft of this manuscript and edited subsequent drafts. All authors contributed to the editing and conceptualization of this paper. 


\section{REFERENCES}

American Psychiatric Association. (2003). Practice guideline for the assessment and treatment of patients with suicidal behaviors. Arlington, VA: American Psychiatric Association.

Bachynski, K., Canham-Chervak, M., Black, S., Dada, E., Millikan, A., \& Jones, B. (2012). Mental health risk factors for suicides in the US Army, 2007-8. Injury Prevention, 18(6), 405-412. doi:10.1136/injuryprev-2011-040112

Bates, M. J., Bowles, S., Hammermeister, J., Stokes, C., Pinder, E., Moore, M., . . Burbelo, G. (2010). Psychological fitness. Mil Med, 175(8, Suppl 1), 21-38.

Beck, A. T., Brown, G. K., \& Steer, R. A. (1997). Psychometric characteristics of the Scale for Suicide Ideation with psychiatric outpatients. Behav Res Ther, 35(11), 1039-1046. doi:10.1016/S0005-7967(97)00073-9

Beck, A. T., \& Steer, R. A. (1991). Manual for Beck Scale for Suicide Ideation. San Antonio, TX: Psychological Corporation.

Beck, A. T., Steer, R. A., \& Ranieri, W. F. (1988). Scale for Suicide Ideation: psychometric properties of a self-report version. J Clin Psychol, 44(4), 499-505. doi:10.1002/10974679(198807)44:4<499::Aid-jclp2270440404>3.0.Co;2-6

Brown, G. K., Beck, A. T., Steer, R. A., \& Grisham, J. R. (2000). Risk factors for suicide in psychiatric outpatients: a 20-year prospective study. J Consult Clin Psychol, 68(3), 371377. doi:10.1037/0022-006X.68.3.371

Brunet, J., McDonough, M. H., Hadd, V., Crocker, P. R., \& Sabiston, C. M. (2010). The Posttraumatic Growth Inventory: an examination of the factor structure and invariance among breast cancer survivors. Psychooncology, 19(8), 830-838. doi:10.1002/pon.1640

Bryan, C. J., \& Cukrowicz, K. C. (2011). Associations between types of combat violence and the acquired capability for suicide. Suicide Life Threat Behav, 41(2), 126-136. doi:10.1111/j.1943-278X.2011.00023.x

Carter, S., Loew, B., Allen, E., Stanley, S., Rhoades, G., \& Markman, H. (2011). Relationships between soldiers' PTSD symptoms and spousal communication during deployment. Journal of Traumatic Stress, 24(3), 352-355. doi:10.1002/jts.20649

Cochrane-Brink, K. A., Lofchy, J. S., \& Sakinofsky, I. (2000). Clinical rating scales in suicide risk assessment. Gen Hosp Psychiatry, 22(6), 445-451. doi:10.1016/S0163-8343(00)00106-7

Connor, K. M., \& Davidson, J. R. (2003). Development of a new resilience scale: the ConnorDavidson Resilience Scale (CD-RISC). Depress Anxiety, 18(2), 76-82. doi:10.1002/da.10113

Davidson, J., Baldwin, D. S., Stein, D. J., Pedersen, R., Ahmed, S., Musgnung, J., . . Rothbaum, B. O. (2008). Effects of venlafaxine extended release on resilience in posttraumatic stress disorder: an item analysis of the Connor-Davidson Resilience Scale. Int Clin Psychopharmacol, 23(5), 299-303. doi:10.1097/YIC.0b013e32830c202d 
Davidson, J. R., Book, S. W., Colket, J. T., Tupler, L. A., Roth, S., David, D., . . Feldman, M. E. (1997). Assessment of a new self-rating scale for post-traumatic stress disorder. Psychol Med, 27(1), 153-160. doi:10.1017/S0033291796004229

Davidson, J. R., Payne, V. M., Connor, K. M., Foa, E. B., Rothbaum, B. O., Hertzberg, M. A., \& Weisler, R. H. (2005). Trauma, resilience and saliostasis: effects of treatment in posttraumatic stress disorder. Int Clin Psychopharmacol, 20(1), 43-48. doi:00004850200501000-00009 [pii]

Davidson, J. R., Stein, D. J., Rothbaum, B. O., Pedersen, R., Szumski, A., \& Baldwin, D. S. (2012). Resilience as a predictor of treatment response in patients with posttraumatic stress disorder treated with venlafaxine extended release or placebo. J Psychopharmacol, 26(6), 778-783. doi:10.1177/0269881111413821

Erbes, C., Westermeyer, J., Engdahl, B., \& Johnsen, E. (2007). Post-traumatic stress disorder and service utilization in a sample of service members from Iraq and Afghanistan. Mil Med, 172(4), 359-363. doi:10.7205/milmed.172.4.359

Friedman, M. J. (2006). Posttraumatic stress disorder among military returnees from Afghanistan and Iraq. Am J Psychiatry, 163(4), 586-593. doi:10.1176/appi.ajp.163.4.586

Gibbons, R. D., Brown, C. H., Hur, K., Davis, J. M., \& Mann, J. J. (2012). Suicidal thoughts and behavior with antidepressant treatment: reanalysis of the randomized placebocontrolled studies of fluoxetine and venlafaxine. Arch Gen Psychiatry, 69(6), 580-587. doi:10.1001/archgenpsychiatry.2011.2048

Gibbons, R. D., Hur, K., Brown, C. H., \& Mann, J. J. (2009). Relationship between antiepileptic drugs and suicide attempts in patients with bipolar disorder. Arch Gen Psychiatry, 66(12), 1354-1360. doi:10.1001/archgenpsychiatry.2009.159

Gibbons, R. D., Hur, K., Brown, C. H., \& Mann, J. J. (2010). Gabapentin and suicide attempts. Pharmacoepidemiol Drug Saf, 19(12), 1241-1247. doi:10.1002/pds.2036

Green, K. T., Calhoun, P. S., Dennis, M. F., \& Beckham, J. C. (2010). Exploration of the resilience construct in posttraumatic stress disorder severity and functional correlates in military combat veterans who have served since September 11, 2001. J Clin Psychiatry, 71(7), 823-830. doi:10.4088/JCP.09m05780blu

Guerra, V. S., Calhoun, P. S., \& Mid-Atlantic Mental Illness Research, Education Clinical Center Workgroup. (2011). Examining the relation between posttraumatic stress disorder and suicidal ideation in an OEF/OIF veteran sample. J Anxiety Disord, 25(1), 12-18. doi:10.1016/j.janxdis.2010.06.025

Hoge, C. W., Auchterlonie, J. L., \& Milliken, C. S. (2006). Mental health problems, use of mental health services, and attrition from military service after returning from deployment to Iraq or Afghanistan. Jama, 295(9), 1023-1032. doi:10.1001/jama.295.9.1023

Hoge, C. W., Castro, C. A., Messer, S. C., McGurk, D., Cotting, D. I., \& Koffman, R. L. (2004). Combat duty in Iraq and Afghanistan, mental health problems, and barriers to care. $N$ Engl J Med, 351(1), 13-22. doi:10.1056/NEJMoa040603 
Isacsson, G., Holmgren, A., Osby, U., \& Ahlner, J. (2009). Decrease in suicide among the individuals treated with antidepressants: a controlled study of antidepressants in suicide, Sweden 1995-2005. Acta Psychiatr Scand, 120(1), 37-44. doi:10.1111/j.16000447.2009.01344.x

Isacsson, G., Reutfors, J., Papadopoulos, F. C., Osby, U., \& AhIner, J. (2010). Antidepressant medication prevents suicide in depression. Acta Psychiatr Scand, 122(6), 454-460. doi:10.1111/j.1600-0447.2010.01561.x

Isacsson, G., Rich, C. L., Jureidini, J., \& Raven, M. (2010). The increased use of antidepressants has contributed to the worldwide reduction in suicide rates. Br J Psychiatry, 196(6), 429433. doi:10.1192/bjp.bp.109.076166

Jakupcak, M., Luterek, J., Hunt, S., Conybeare, D., \& McFall, M. (2008). Posttraumatic stress and its relationship to physical health functioning in a sample of Iraq and Afghanistan War veterans seeking postdeployment VA health care. J Nerv Ment Dis, 196(5), 425-428. doi:10.1097/NMD.0b013e31817108ed

Kaplan, M. S., Huguet, N., McFarland, B. H., \& Newsom, J. T. (2007). Suicide among male veterans: a prospective population-based study. J Epidemiol Community Health, 61(7), 619-624. doi:10.1136/jech.2006.054346

Leve, L. D., Fisher, P. A., \& Chamberlain, P. (2009). Multidimensional treatment foster care as a preventive intervention to promote resiliency among youth in the child welfare system. J Pers, 77(6), 1869-1902. doi:10.1111/j.1467-6494.2009.00603.x

Lockwood, L. E., \& Youssef, N. A. (2017). Systematic review of epigenetic effects of pharmacological agents for bipolar disorders. Brain Sci, 7(11), 154. doi:10.3390/brainsci7110154

McDonald, S. D., Beckham, J. C., Morey, R. A., \& Calhoun, P. S. (2009). The validity and diagnostic efficiency of the Davidson Trauma Scale in military veterans who have served since September 11th, 2001. J Anxiety Disord, 23(2), 247-255. doi:10.1016/j.janxdis.2008.07.007 [doi]

Milliken, C. S., Auchterlonie, J. L., \& Hoge, C. W. (2007). Longitudinal assessment of mental health problems among active and reserve component soldiers returning from the Iraq war. Jama, 298(18), 2141-2148. doi:10.1001/jama.298.18.2141

Nrugham, L., Holen, A., \& Sund, A. M. (2010). Associations between attempted suicide, violent life events, depressive symptoms, and resilience in adolescents and young adults. J Nerv Ment Dis, 198(2), 131-136. doi:10.1097/NMD.0b013e3181cc43a2

O'Donnell, D. A., Schwab-Stone, M. E., \& Muyeed, A. Z. (2002). Multidimensional resilience in urban children exposed to community violence. Child Dev, 73(4), 1265-1282. doi:10.1111/1467-8624.00471

Oquendo, M. A., Galfalvy, H. C., Currier, D., Grunebaum, M. F., Sher, L., Sullivan, G. M., . . Mann, J. J. (2011). Treatment of suicide attempters with bipolar disorder: a randomized 
clinical trial comparing lithium and valproate in the prevention of suicidal behavior. Am J Psychiatry, 168(10), 1050-1056. doi:10.1176/appi.ajp.2011.11010163

Panagioti, M., Gooding, P., \& Tarrier, N. (2009). Post-traumatic stress disorder and suicidal behavior: a narrative review. Clin Psychol Rev, 29(6), 471-482. doi:10.1016/j.cpr.2009.05.001

Pietrzak, R. H., Goldstein, M. B., Malley, J. C., Rivers, A. J., Johnson, D. C., \& Southwick, S. M. (2010). Risk and protective factors associated with suicidal ideation in veterans of Operations Enduring Freedom and Iraqi Freedom. J Affect Disord, 123(1-3), 102-107. doi:10.1016/j.jad.2009.08.001

Reivich, K. J., Seligman, M. E., \& McBride, S. (2011). Master resilience training in the U.S. Army. Am Psychol, 66(1), 25-34. doi:10.1037/a0021897

Roy, A. (2011). Combination of family history of suicidal behavior and childhood trauma may represent correlate of increased suicide risk. J Affect Disord, 130(1-2), 205-208. doi:10.1016/j.jad.2010.09.022

Roy, A., Carli, V., \& Sarchiapone, M. (2011). Resilience mitigates the suicide risk associated with childhood trauma. J Affect Disord, 133(3), 591-594. doi:10.1016/j.jad.2011.05.006

Sarchiapone, M., Carli, V., Cuomo, C., \& Roy, A. (2007). Childhood trauma and suicide attempts in patients with unipolar depression. Depress Anxiety, 24(4), 268-272. doi:10.1002/da.20243

Steer, R. A., Rissmiller, D. J., Ranieri, W. F., \& Beck, A. T. (1993). Dimensions of suicidal ideation in psychiatric inpatients. Behav Res Ther, 31(2), 229-236. doi:10.1016/00057967(93)90090-H

Wright, M. O., Fopma-Loy, J., \& Fischer, S. (2005). Multidimensional assessment of resilience in mothers who are child sexual abuse survivors. Child Abuse Negl, 29(10), 1173-1193. doi:10.1016/j.chiabu.2005.04.004

Youssef, N. A., Green, K. T., Dedert, E. A., Hertzberg, J. S., Calhoun, P. S., Dennis, M. F., \& Beckham, J. C. (2013). Exploration of the influence of childhood trauma, combat exposure, and the resilience construct on depression and suicidal ideation among U.S. Iraq/Afghanistan era military personnel and veterans. Arch Suicide Res, 17(2), 106-122. doi:10.1080/13811118.2013.776445

Yu, X. N., Lau, J. T., Mak, W. W., Zhang, J., \& Lui, W. W. (2011). Factor structure and psychometric properties of the Connor-Davidson Resilience Scale among Chinese adolescents. Compr Psychiatry, 52(2), 218-224. doi:10.1016/j.comppsych.2010.05.010

Zivin, K., Kim, H. M., McCarthy, J. F., Austin, K. L., Hoggatt, K. J., Walters, H., \& Valenstein, M. (2007). Suicide mortality among individuals receiving treatment for depression in the Veterans Affairs health system: associations with patient and treatment setting characteristics. Am J Public Health, 97(12), 2193-2198. doi:10.2105/AJPH.2007.115477 


\section{Chapter 6 Racial/Ethnic Differences in the Association of Childhood Adversities With Depression and the Role of Resilience}

Nagy A. Youssef, ${ }^{1 *}$ Daniel Belew, ${ }^{2}$ Guang Hao, ${ }^{3}$ Xiaoling Wang, ${ }^{3}$ Frank A. Treiber, ${ }^{4}$ Michael Stefanek, ${ }^{5}$ Mark Yassa, ${ }^{1}$ Elizabeth Boswell, ${ }^{1}$ W. Vaughn McCall, ${ }^{1}$ Shaoyong Su ${ }^{3 *}$

${ }^{1}$ Department of Psychiatry \& Health Behavior, Medical College of Georgia, Augusta University, Augusta, GA.

${ }^{2}$ Department of Surgery, Medical College of Georgia, Augusta University, USA.

${ }^{3}$ Department of Pediatrics, Medical College of Georgia, Augusta University, Augusta, GA.

${ }^{4}$ Technology Applications Center for Healthful Lifestyles, Colleges of Nursing and Medicine, Medical University of South Carolina, Charleston, SC.

${ }^{5}$ Department of Psychological Sciences, College of Science and Mathematics, Augusta University, Augusta, GA.

Published as: Youssef NA, Belew D, Hao G, Wang X, Treiber FA, Stefanek M, Yassa M, Boswell E, McCall WV, Su S. Racial/ethnic differences in the association of childhood adversities with depression and the role of resilience. J Affect Disord. 2017 Jan 15;208:577-581. doi: 10.1016/j.jad.2016.10.024. Epub 2016 Oct 24. PubMed PMID: 27806896; PubMed Central PMCID: PMC5154886. https://www.sciencedirect.com/science/article/pii/S0165032716310783?via\%3Dihub 


\section{Abstract}

Background: Adverse childhood experiences (ACE) including childhood abuse and trauma increase depressive symptoms. The role of resilience and how it interacts with both ACEs and the potential development of depressive symptoms, including how race and ethnicity moderate these effects, are much less studied. The aims of this study were to examine: 1) whether there is a dose-response relationship between trauma and depressive symptoms; 2) whether early trauma affected European Americans (EA) and African Americans (AA) in a similar fashion; and 3) whether resilience mitigates the effect of trauma.

Methods: The present study comprised a cross-sectional study of subjects from a longitudinal cohort. All subjects were 19 years or older with traumatic experiences prior to age 18 . Subjects were assessed for depressive symptoms as well as resilience.

Results: In 413 subjects enrolled, ACEs were significantly associated with depression severity in a dose response fashion $(p<0.001)$. Notably, AAs had lower depression scores at low to moderate levels of ACEs than EAs, but reported comparable levels of depression with severe exposure to ACEs (pInteraction=0.05). In both EAs and AAs, young adults with high and medium levels of resilience showed less depressive symptoms compared to those with low resilience $(p<0.05)$.

Limitations: The cross-sectional design, possibility of other confounders, and potential for recall bias of this study.

Conclusion: While ACEs were significantly associated with severity of depression in a doseresponse fashion, higher resilience mitigated the impact of childhood adversities on depressive symptoms in young adults. The results are encouraging, and guides research for therapeutics to boost resilience.

\section{Keywords}

Adverse childhood experiences, Depressive symptoms, Resilience, Race/ethnicity 


\section{INTRODUCTION}

Adverse childhood experiences (ACEs), characterized by childhood maltreatment and household dysfunction, have been associated with multiple physical health impairments in adulthood, including heart disease, obesity and type 2 diabetes (Felitti et al., 1998). Trauma exposure during early life has also been related to mental health issues later in life, including depression (Schulz et al., 2014; Wingo et al., 2010; Youssef, Green, Dedert, et al., 2013) and suicidality (Youssef, Green, Beckham, \& Elbogen, 2013). For example, Youssef et al. found that childhood trauma exposure was significantly associated with both depressive symptoms and suicidal ideation in 1488 military personnel and veterans while controlling for the effects of combat exposure and posttraumatic stress disorder (PTSD) (Youssef, Green, Dedert, et al., 2013). Resilience, defined as qualities that enable one to thrive in the face of adversity, (Connor \& Davidson, 2003) has been negatively associated with depressive symptoms and suicidal ideation, suggesting a protective effect (Youssef, Green, Beckham, et al., 2013; Youssef, Green, Dedert, et al., 2013). However, the role of resilience and how it interacts with both ACEs and the potential development of depressive symptoms, including how race and ethnicity moderate these effects, are much less studied.

The aims of the present study were to examine the following: 1) whether there is a doseresponse relationship between number of trauma exposure in early life and depressive symptoms in young adulthood; 2) whether early trauma affected European Americans (EA) and African Americans (AA) in a similar fashion; and 3) whether resilience mitigates the effect of early trauma on the development of depression.

\section{METHODS}

\section{Subjects}

The present study is comprised of subjects from a longitudinal cohort that was established in 1989 to study the development of cardiovascular risk factors. All the subjects were recruited from the southeastern United States, who met the following criteria: (1) aged 5 to 16 years in 1989; (2) African or European ancestry; and (3) apparently healthy based on parental report of the child's medical history. Participants were classified as AAs if both parents reported being of African heritage and they considered themselves and their child to be AA, black, or AfroAmerican. Participants were classified as EAs if both parents reported that they were of European ancestry and they considered themselves and their child to be EA, white, or Caucasian, and not of Hispanic, Native American, or Asian descent. The study design and selection criteria have been described previously (Su et al., 2015).

At visit 15 (initiated at 2008), all subjects were 19 years or older and their traumatic experiences prior to age 18 were assessed by using the ACE questionnaire. Of 432 who were enrolled at visit 15, 413 completed the ACE questionnaire, including 192 EAs ( 99 males and 93 females) and 221 AAs (93 males and 128 females). There were no demographical differences (including age, gender, and ethnicity) between subjects who were included in the analysis $(\mathrm{N}=413)$ and those excluded $(\mathrm{N}=19)$ due to missing values.

\section{Demographics Measurements}

Demographic data were collected. Childhood socioeconomic status (SES) was assessed by Hollingshead Four Factor Social Status Index on the basis of parental education level and 
occupation (Hollingshead, 1975). The Hollingshead scores ranged from 14 to 66, with a higher value indicating a higher SES.

\section{Assessment of Adverse Childhood Experiences}

The assessment of participants' exposure to ACEs covered the first 18 years of their lives. The ACE questionnaire consists of 28 items divided into 3 categories and 10 subscales, including childhood abuse (emotional, physical and sexual), neglect (emotional and physical), and growing up with household dysfunction (substance abuse, mental illness, domestic violence, criminal household member, and parental marital discord). The definition of ACEs has been described previously (Su et al., 2015). The ACE score (the number of 10 ACE subscales reported) was used to assess the cumulative effect of multiple ACEs, by classifying respondents into four groups: no exposure (0 ACEs, $\mathrm{N}=126)$, mild (1-2 ACEs, $\mathrm{N}=157)$, moderate (3-4 ACEs, $\mathrm{N}=75)$ and severe ( $\geq 5$ ACEs, $\mathrm{N}=55$ ) exposure.

\section{Assessment of Depressive Symptoms}

The Beck Depression Inventory (BDI) was used to assess depressive symptoms, a standardized scale providing a continuous measure of depressive symptoms (Beck, Steer, \& Brown, 1996). This self-report instrument includes 21 items. Participants rate the severity of each symptom from 0 to 3 . It has been used extensively in community samples and has satisfactory test-retest and internal consistency reliability.

\section{Assessment of Resilience}

Resilience was assessed using the Connor Davidson Resilience Scale (CD-RISC), which is a 25-item self-reported instrument that measures the ability to cope with stress and adversity, such as self-confidence, self-efficacy, self-control, optimism and spirituality/autonomy (Connor $\&$ Davidson, 2003). The items are scored on a five-point Likert scale, ranging from "not true at all" (scored 0) to "true nearly all the time" (scored 4). The scale rates participants over the past month with a total score of the CD-RISC varying from 0 to 100, with higher scores reflecting higher resilience.

\section{Statistical Analysis}

All analyses were done using STATA software. Sociodemographic variables were characterized with descriptive statistics ( $\mathrm{t}$-test for continuous traits and $\chi 2$-test for categorical traits). Multiple linear regression models were used to estimate the associations of ACE scores and resilience (CD-RISC scores) with depressive symptoms (BDI scores). Covariates included age, race, sex, BMI and childhood SES (Hollingshead index). The ethnicity and gender differences were further tested by including the interaction of ACE scores with race and sex, respectively. Statistical significance required a two-sided $p$-value of $\leq 0.05$. We conducted a priori power calculation using the G*Power software (Faul, Erdfelder, Buchner, \& Lang, 2009). At a two-tailed $\alpha$ level of 0.05 , the required sample size is $N=408$ to achieve a sufficient power of 0.8 for identifying an effect size as small as 0.027 (Cohen $\left.f^{2}\right)$. Therefore, the current study $(\mathrm{N}=413)$ should have sufficient power to identify small to medium effects.

\section{RESULTS}


A total of 413 subjects were included in the present study. The mean age ( $\pm S D$ ) was 28.3 \pm 3.1 (age range: $19.4-36.8$ ). Table 1 presents the descriptive characteristics of the subjects by ethnicity and gender. African Americans (AA) had significantly greater BMI and lower childhood SES than European Americans (EA) ( $p \leq 0.001)$. Men had lower BMI than women $(p=0.003)$. The mean score on the BDI $( \pm S D)$ was $6.7 \pm 7.7$, with a minimum of 0 and a maximum of 39. The mean score on the CD-RISC ( \pm SD) was $75.5 \pm 13.9$, with a minimum of 27 and a maximum of 100. No significant differences on the BDI score or CD-RISC score were found between AAs and EAs, or between men and women.

\section{Prevalence of ACEs}

The prevalence of each individual ACE subscale and ACE scores are shown in Table 2, with the lowest prevalence of physical neglect (9.0\%) and the highest prevalence of parental marital discord (38.7\%). Compared to women, men reported much higher rate of experiencing physical neglect during childhood (15.1\% versus 3.6\%). Significant racial differences are evident in some of the ACE subscales (Table 2). Approximately 1 in 3 EA respondents reported that they lived with a household member who was mentally ill or attempted suicide, a rate twice as high as the rate reported by $A A$ respondents (35.4\% versus 17.2\%). However, AA respondents reported higher rates of other ACE subscales than EA respondents. For example, 1 in 8 AA respondents reported experiencing physical neglect as a child, a rate 2 times higher than the rate reported by EA respondents (12.2\% versus 5.2\%). AA respondents also reported higher rates of (a) witnessing domestic violence ( $24 \%$ versus $17.2 \%$ ), (b) a household member who went to prison (21.3\% versus $9.9 \%$ ) and (c) parental marital discord (47.1\% versus $29.2 \%)$. Overall, the prevalence of any ACE event is relatively higher in AAs than that in EAs (73.3\% versus $65.1 \%, p=0.07)$. In summary, $69.5 \%$ of respondents reported at least one exposure to ACEs, with mild (1-2 ACEs), moderate (3-4 ACEs), and severe ( $\geq 5$ ACEs) exposure to ACEs representing $38.0 \%, 18.2 \%$ and $13.3 \%$, respectively (Table 2 ). The prevalence of ACE scores was not significantly different between men and women, although men showed a higher rate of severe exposure to ACEs than women (16.6\% versus $10.4 \%$ ). AAs had a relatively higher prevalence of moderate and severe exposure to ACEs compared to EAs ( $21.3 \%$ versus $14.6 \%$ and $14.9 \%$ versus $11.4 \%$ respectively).

\section{Effects of ACEs on depressive symptoms}

The effects of each individual ACE on depressive symptoms are shown in Table 3 by gender and race. This table presents beta coefficients from regression models in which BDI score was regressed on one ACE, controlling for age, gender, race, BMI and childhood SES. Seven of ten ACEs were significantly associated with reports of higher depressive symptoms in both men and women, as well as in EAs and AAs. Women were more vulnerable to emotional neglect compared to men (beta coefficients: 8.0 versus 2.5; interaction $p=0.02$ ), while men reported higher depressive symptoms than women if some household member went to prison (beta coefficients: 5.2 versus 1.1 ; interaction $p=0.03$ ). The most frequently experienced ACE - parental marital discord - was not related to depressive symptoms in any gender or race group. The impacts of ACEs on depressive symptoms were generally stronger in EAs than in AAs for a given ACE, although they were not statistically different.

However, the cumulative effects of ACEs on depressive symptoms were significantly different between $E A$ and $A A$ respondents (interaction $p=0.05$ ). As shown in Table 4, after 
controlling for age, gender, BMI and childhood SES, the EA participants who reported mild, moderate, and severe exposure to ACEs (1-2, 3-4, 5+) had an average increase of 2.1, 7.3 and 11.1 points on BDI scores compared to those with no exposure to ACEs $(p=0.05,<0.001$ and $<0.001$ respectively), while the AA participants at the same ACE exposure levels showed an average increase of $0.8,3.3$ and 12.6 points on BDI scores compared to their counterparts without ACEs ( $p=0.5,0.02$ and $<0.001$ respectively). There was no significant gender-by-ACE interaction, suggesting that the cumulative effect of ACEs on depressive symptoms was similar between men and women.

\section{Role of resilience}

We next examined the role of resilience in the relationship between ACE exposure and depression. For a visual conceptualization of the interaction between childhood adversities and resilience, we divided resilience into 3 categories based on the percentile of the CD-RISC score: high resilience: CD-RISC score $>75$ th percentile, medium resilience: CDRISC score $>25$ th percentile and $\leq 75$ th percentile, and low resilience: CD-RISC score $\leq 25$ th percentile. We found a significant interaction between ACE scores and resilience on depressive symptoms $(p<0.05)$. Given similar levels of childhood adversity exposure, in both EAs and AAs, the high and medium resilience group had lower BDI scores compared to the low resilience group (Figure 1), suggesting that resilience mitigates the tendency for developing depressive symptoms in young adults with a history of childhood adversities. There is no three-way interaction of race, ACE scores and CDRISC scores on depressive symptoms, indicating that resilience is a protective factor in both EA and AA populations.

\section{DISCUSSION}

This study has several findings that were expected and some that were not. We found that exposure to ACEs was significantly associated with severity of depression in a dose-response fashion. Given similar levels of childhood trauma exposure, young adults with high and medium levels of resilience showed less depressive symptoms compared to those with low resilience, as previously reported in both active duty service members and veterans (Youssef, Green, Dedert, et al., 2013). Our results replicated and generalized previous findings to the civilian young adult population.

However, an interesting finding in this study is that depression was only markedly higher in AAs with severe ACEs but not in mild or moderate ACEs; showing a significant interaction by race $(p=0.05)$. AAs in general have less depressive scores at low to moderate level of ACEs than EAs, except for the severe exposure to ACEs where both races were more comparable in the severity of depression.

Our results are consistent with previous literature in several ways. For example, in a community-based ethnically diverse cohort of young adults in South Florida, Turner and Lloyd observed that AAs had generally experienced more adverse events than other ethnic groups but showed relatively lower prevalence of depressive disorders (Turner \& Lloyd, 2004). In a longitudinal survey in young adults, Schilling et al. found that when racial/ethnic differences exist, young EAs consistently exhibit greater vulnerability to ACEs (Schilling, Aseltine, \& Gore, 2007). One possible explanation is the potential social or cultural differences in resilience between two ethnic groups (Rutter, 1996). 
However, the overall resilience scores did not differ between the two ethnic groups. Further adjustment for resilience scores did not change the results, suggesting that this racial/ethnic difference may not be attributable to the social or cultural differences in resilience. Another possible explanation is that individuals from minority backgrounds may be less willing to disclose mental health symptoms, which are often stigmatized in AA communities (U.S. Department of Health and Human Services \& U.S. Public Health Service, 2001). However, it is of note that AAs exhibited high depressive scores that were comparable to EAs as the number of stressful childhood events exceeds 5 or more, suggesting that high levels of cumulative stress can have fairly equivalent negative effect on mental health for both AAs and EAs.

The protective role of resilience against stress and depression has been reported previously. For instance, a cross-sectional study of 792 predominantly African American men and women found that resilience moderated depressive symptom severity in individuals exposed to childhood abuse or other trauma (Wingo et al., 2010). Consistent with these findings, we also observed a significant interaction between ACE scores and resilience on depressive symptoms. In both EAs and AAs, young adults exposed to severe ACEs but with high levels of resilience showed less depressive symptoms compared to those with low resilience, suggesting that resilience mitigates the tendency for developing depressive symptoms in young adults with a history of childhood adversities.

There are several limitations to consider when interpreting the results of this study. First, given the cross-sectional design, the results cannot be interpreted in a cause-effect fashion, but only associations can be shown. Second, due to the observational design, the possibility of other potential confounding factors cannot be totally eliminated. Third, the potential for recall bias of past childhood experiences is inevitable in this type of study. However, good test-retest reliability of recall of past childhood trauma, and stability over time has been shown (Dube, Williamson, Thompson, Felitti, \& Anda, 2004). Examination against documented cases of childhood trauma showed that inconsistencies are likely to be due to under-reporting rather than over-reporting of the trauma (Della Femina, Yeager, \& Lewis, 1990; Dube et al., 2004; Hardt \& Rutter, 2004). However, we did not collect information of an exact time frame when the childhood traumatic events occurred.

\section{CONCLUSION}

Exposure to ACEs was significantly associated with depression severity, while resilience mitigated the impact of childhood adversities on developing depressive symptoms in young adults. AAs exposed to low to moderate levels of ACEs had fewer depressive symptoms than EAs while at high levels of stress they exhibited similar severity of depression compared to EAs. This study provides useful information in the clinical assessment of patients with ACEs and depression. The results of our study are encouraging, and guide further research for promising therapies to boost resilience and potentially decrease depressive symptoms. 
Acknowledgment: We thank Grant Zackary for his assistance in preparation of a subsection of this manuscript. We acknowledge the continued cooperation and participation of the members of the study and their families and thank the tireless staff at the Georgia Prevention Institute.

Funding: This research was supported in part by the National Institutes of Health Program Project Grant on Stress-Related Mechanisms of Hypertensive Risk (P01 HL69999 to FAT). SS is funded by the American Heart Association (09SDG2140117) and the NIH (HL106333-01A1 and HL12557701).

Declaration of interest: Authors declare no conflict of interest relevant to this study.

Contributors: Nagy Youssef contributed to the conceptualization of the analysis and interpretation of the results and wrote the first draft of this manuscript and subsequent revisions. Shaoyong Su contributed to the performance of the statistical analysis and interpretation of the results and helped in writing the first draft of this manuscript and subsequent revisions. Daniel Belew, Guang Hao, Xiaoling Wang, Frank A. Treiber, Michael Stefanek, Mark Yassa, Elizabeth Boswell, W. Vaughn McCall helped in writing and editing this manuscript and contributed intellectually to this manuscript. All authors have approved the final article. 
Table 1

Descriptive characteristics of study participants (total $\mathrm{N}=413$ ).

\begin{tabular}{lllllll}
\hline Characteristic & Men & Women & $P_{\text {gender }}$ & EA & AA & $P_{\text {race }}$ \\
\hline $\mathrm{N}$ & 192 & 221 & & 192 & 221 & \\
Age, y & 28.2 & $28.5(2.9)$ & 0.45 & 28.0 & 28.7 & 0.025 \\
& $(3.3)$ & & & $(3.0)$ & $(3.1)$ & \\
Body mass index, & 29.2 & $31.5(9.8)$ & 0.005 & 29.0 & 31.7 & 0.002 \\
$\quad$ kg/m & $(6.6)$ & & & $(7.8)$ & $(9.0)$ & \\
Hollingshead & 41.9 & $40.6(13.1)$ & 0.33 & 45.2 & 37.7 & $<0.001$ \\
$\quad$ index & $(13.9)$ & & & $(12.3)$ & $(13.6)$ & \\
Depression & 6.6 & $6.8(7.7)$ & 0.77 & 6.5 & 6.9 & 0.60 \\
$\quad$ symptoms & $(7.8)$ & & & $(7.4)$ & $(8.0)$ & \\
$\quad(B D I$ score $)$ & & & & & & \\
Resilience (CD- & 75.4 & $75.7(13.3)$ & 0.86 & 74.4 & 76.5 & 0.12 \\
$\quad$ RISC score) & $(14.6)$ & & & $(13.4)$ & $(14.3)$ & \\
\hline
\end{tabular}

Means and standard deviations (SD) are shown.

EA: European American; AA: African American; BDI: Beck Depression Inventory; CDRISC: Connor-Davidson Resilience Scale. 


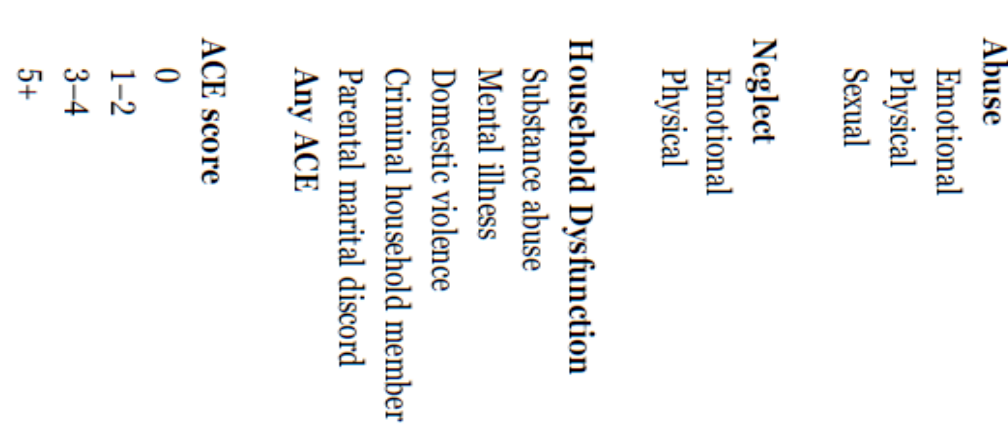

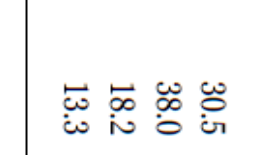

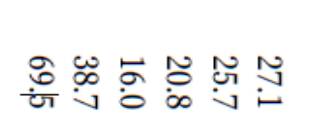

밤

ڤ̆

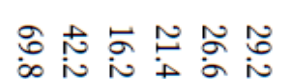

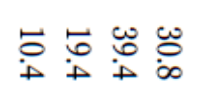

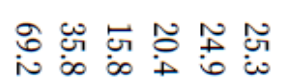

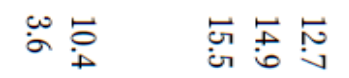

응영용

융잉 융ㅇㅇㅇ잉

융융 엉윱

芦苦㟔苦

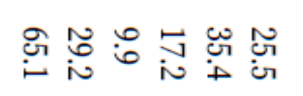

iv

ज心

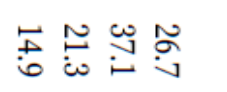

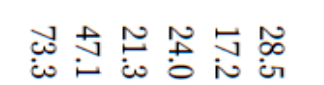

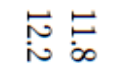

10.0
$\infty$
$\infty$
0

ن잉ㅇㅇ

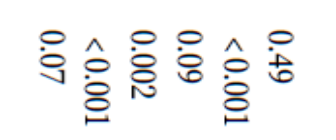

兽 엉

입융

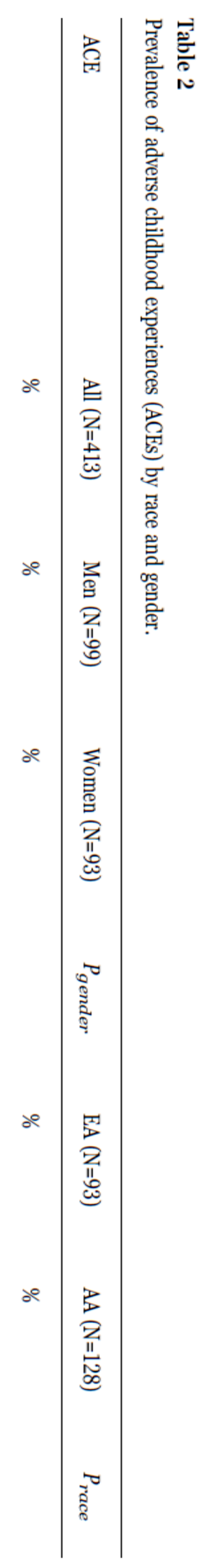




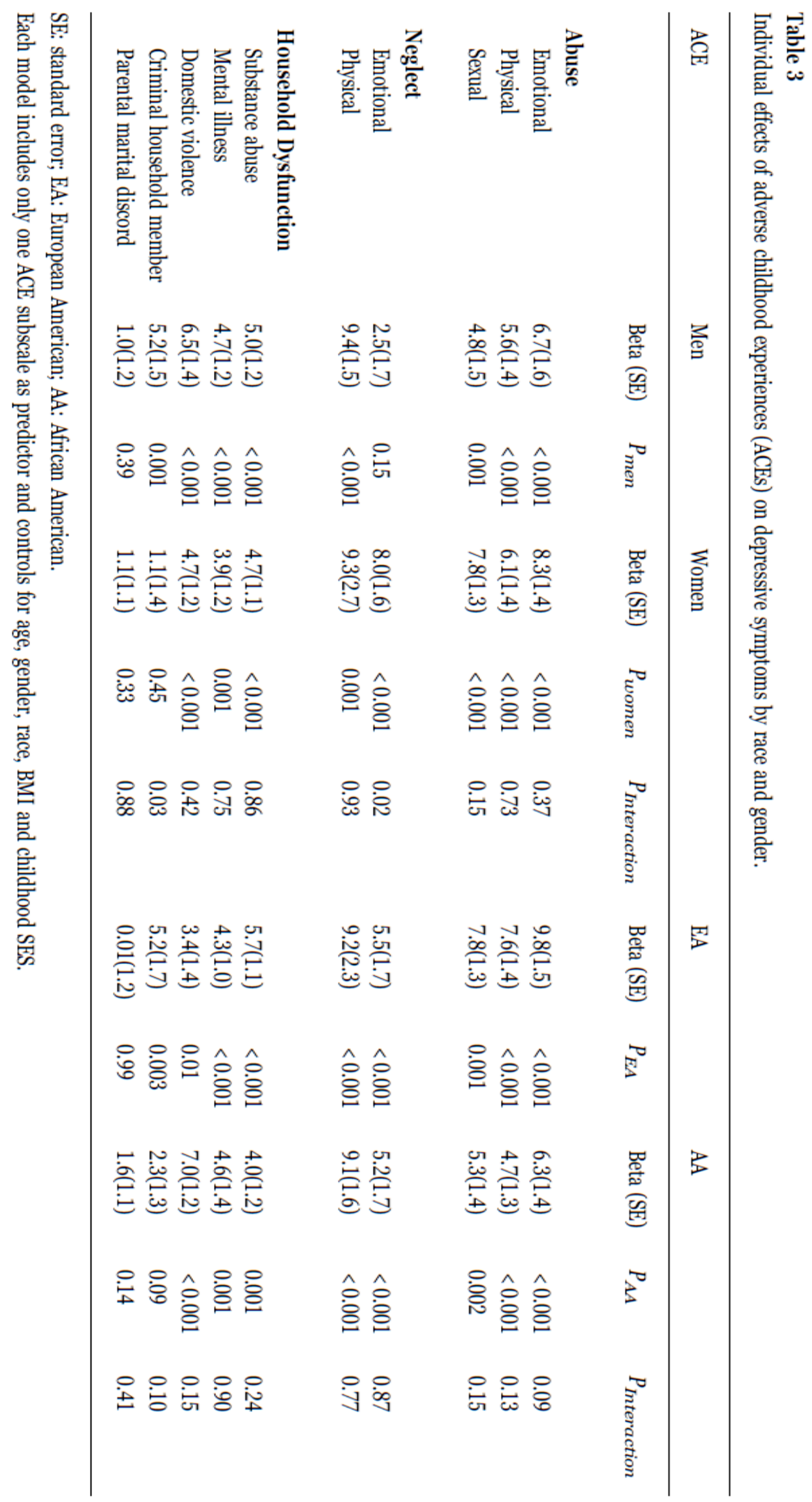




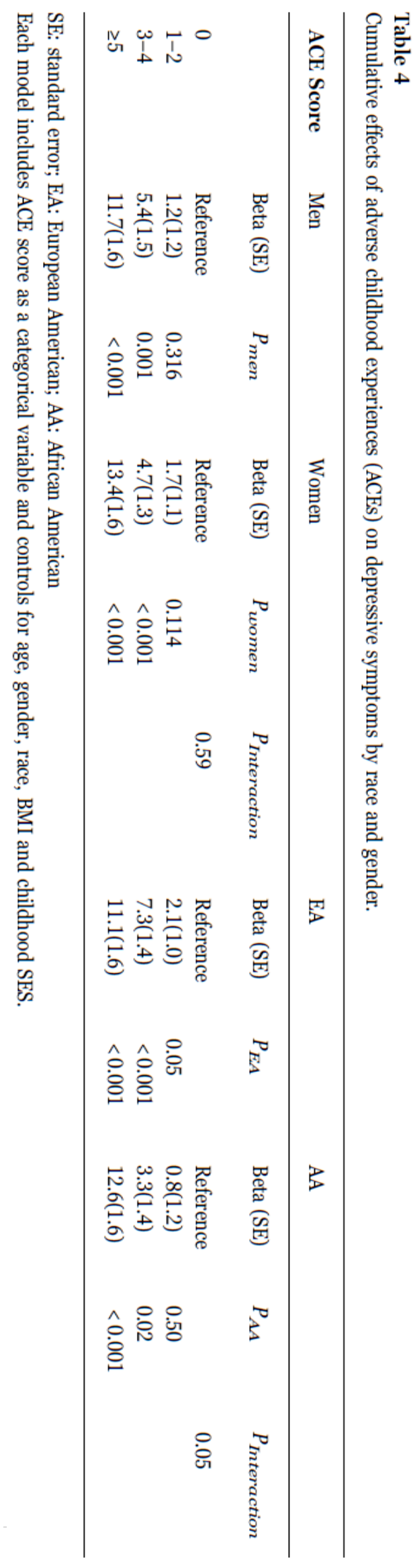



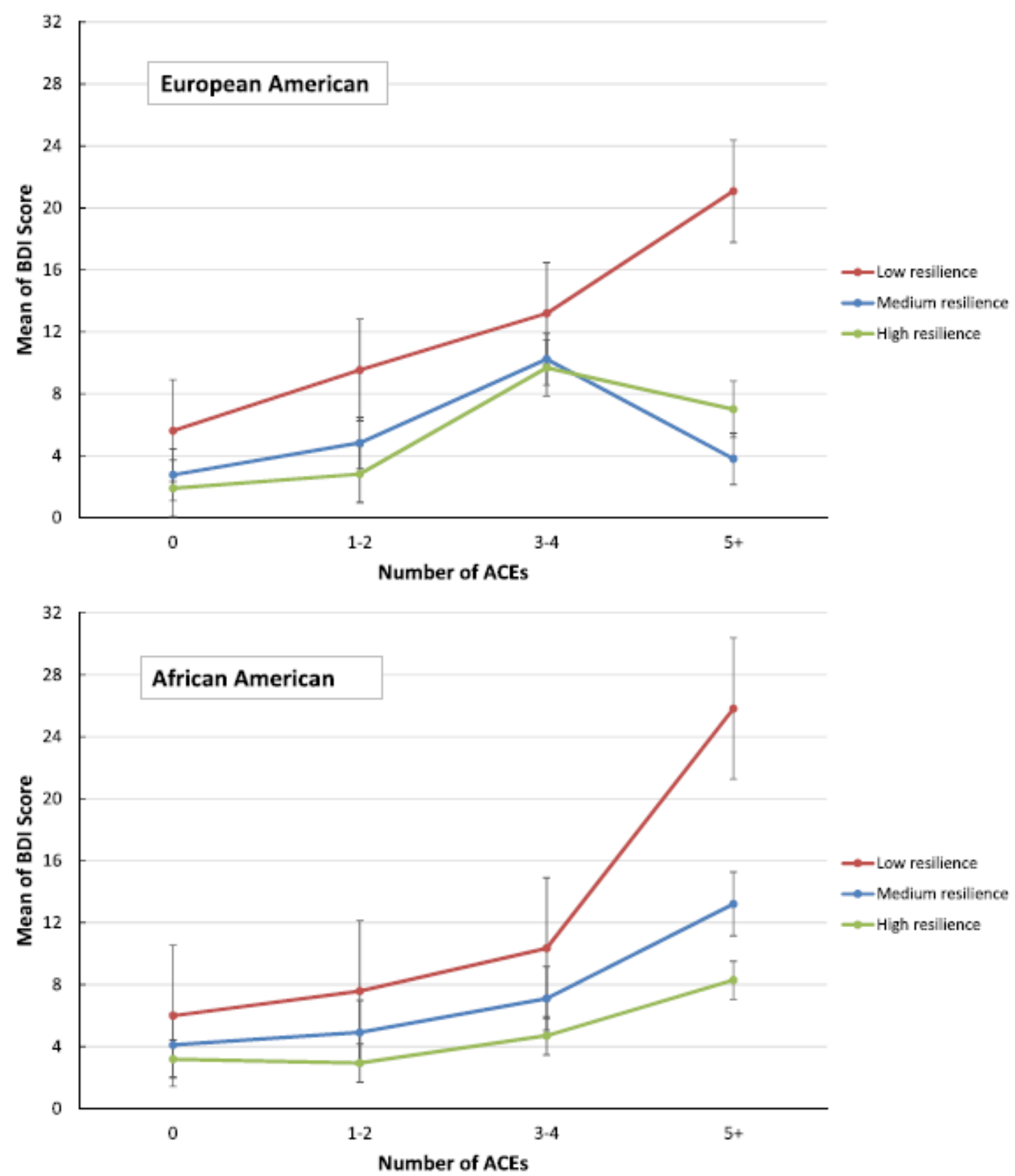

Fig. 1. Depressive symptoms according to ACE scores by Conner-Davidson resilience scale (total score $\leq 25$ th percentile, $>25$ th and $<75$ th percentile, and $\geq 75$ th percentile) and race (European American or African American). 


\section{REFERENCES}

Beck, A. T., Steer, R. A., \& Brown, G. (1996). Beck Depression Inventory-II. San Antonio, TX: The Psychological Corporation.

Connor, K. M., \& Davidson, J. R. (2003). Development of a new resilience scale: the ConnorDavidson Resilience Scale (CD-RISC). Depress Anxiety, 18(2), 76-82. doi:10.1002/da.10113

Della Femina, D., Yeager, C. A., \& Lewis, D. O. (1990). Child abuse: adolescent records vs. adult recall. Child Abuse Negl, 14(2), 227-231. doi:10.1016/0145-2134(90)90033-P

Dube, S. R., Williamson, D. F., Thompson, T., Felitti, V. J., \& Anda, R. F. (2004). Assessing the reliability of retrospective reports of adverse childhood experiences among adult HMO members attending a primary care clinic. Child Abuse Negl, 28(7), 729-737. doi:10.1016/j.chiabu.2003.08.009

Faul, F., Erdfelder, E., Buchner, A., \& Lang, A. G. (2009). Statistical power analyses using G*Power 3.1: tests for correlation and regression analyses. Behav Res Methods, 41(4), 1149-1160. doi:10.3758/BRM.41.4.1149

Felitti, V. J., Anda, R. F., Nordenberg, D., Williamson, D. F., Spitz, A. M., Edwards, V., ... Marks, J. S. (1998). Relationship of childhood abuse and household dysfunction to many of the leading causes of death in adults. The Adverse Childhood Experiences (ACE) Study. Am J Prev Med, 14(4), 245-258. doi:10.1016/S0749-3797(98)00017-8

Hardt, J., \& Rutter, M. (2004). Validity of adult retrospective reports of adverse childhood experiences: review of the evidence. J Child Psychol Psychiatry, 45(2), 260-273. doi:10.1111/j.1469-7610.2004.00218.x

Hollingshead, A. B. (1975). Four Factor Index of Social Status [unpublished working paper]. Yale University Department of Sociology. New Haven, CT.

Rutter, M. (1996). Stress research: accomplishments and tasks ahead. In R. J. Haggerty, L. R. Sherrod, N. Garmezy, \& M. Rutter (Eds.), Stress, risk, and resilience in children and adolescents: processes, mechanisms, and interventions. New York: Cambridge University Press.

Schilling, E. A., Aseltine, R. H., Jr., \& Gore, S. (2007). Adverse childhood experiences and mental health in young adults: a longitudinal survey. BMC Public Health, 7, 30. doi:10.1186/1471-2458-7-30

Schulz, A., Becker, M., Van der Auwera, S., Barnow, S., Appel, K., Mahler, J., . . . Grabe, H. J. (2014). The impact of childhood trauma on depression: does resilience matter? Population-based results from the Study of Health in Pomerania. J Psychosom Res, 77(2), 97-103. doi:10.1016/j.jpsychores.2014.06.008

Su, S., Wang, X., Pollock, J. S., Treiber, F. A., Xu, X., Snieder, H., . . Harshfield, G. A. (2015). Adverse childhood experiences and blood pressure trajectories from childhood to young 
adulthood: the Georgia Stress and Heart Study. Circulation, 131(19), 1674-1681. doi:10.1161/CIRCULATIONAHA.114.013104

Turner, R. J., \& Lloyd, D. A. (2004). Stress burden and the lifetime incidence of psychiatric disorder in young adults: racial and ethnic contrasts. Arch Gen Psychiatry, 61(5), 481488. doi:10.1001/archpsyc.61.5.481

U.S. Department of Health and Human Services, \& U.S. Public Health Service. (2001). Mental Health: Culture, Race, and Ethnicity - A Supplement to Mental Health: A Report of the Surgeon General. Retrieved from Rockville, MD: http://www.ncbi.nIm.nih.gov/books/NBK44243/

Wingo, A. P., Wrenn, G., Pelletier, T., Gutman, A. R., Bradley, B., \& Ressler, K. J. (2010). Moderating effects of resilience on depression in individuals with a history of childhood abuse or trauma exposure. J Affect Disord, 126(3), 411-414. doi:10.1016/j.jad.2010.04.009

Youssef, N. A., Green, K. T., Beckham, J. C., \& Elbogen, E. B. (2013). A 3-year longitudinal study examining the effect of resilience on suicidality in veterans. Ann Clin Psychiatry, 25(1), 59-66. Retrieved from http://www.ncbi.nlm.nih.gov/pubmed/23376871

Youssef, N. A., Green, K. T., Dedert, E. A., Hertzberg, J. S., Calhoun, P. S., Dennis, M. F., \& Beckham, J. C. (2013). Exploration of the influence of childhood trauma, combat exposure, and the resilience construct on depression and suicidal ideation among U.S. Iraq/Afghanistan era military personnel and veterans. Arch Suicide Res, 17(2), 106-122. doi:10.1080/13811118.2013.776445 



\section{Chapter 7 Moral Injury, Post-Traumatic Stress Disorder, and Religious Involvement in U.S. Veterans}

Nagy A. Youssef, MD, ${ }^{1,2}$ Elizabeth Boswell, BA, ${ }^{1,2}$ Shashana Fiedler, MPH, ${ }^{1,2}$ Rebecca Jump, PhD, ${ }^{2}$ Elizabeth Lee, BS, ${ }^{1}$ Mark Yassa, BS, ${ }^{1}$ Fazal Khan, MD, ${ }^{2}$ Ananda Pathiraja, MD, ${ }^{2}$ David Rizk, BS, ${ }^{5}$ Michelle Pearce, PhD, ${ }^{3,4}$ Keisha O'Garo, PsyD, ABPP, ${ }^{4}$ Joseph M. Currier, PhD, ${ }^{5}$ Harold G. Koenig, $\mathrm{MD}^{4,6,7}$

${ }^{1}$ Medical College of Georgia, Augusta University, Augusta, Georgia.

${ }^{2}$ Charlie Norwood VA Medical Center, Augusta, Georgia.

${ }^{3}$ University of Maryland School of Medicine, Baltimore, Maryland.

${ }^{4}$ Duke University Medical Center, Durham, North Carolina.

${ }^{5}$ University of South Alabama, Mobile, Alabama.

${ }^{6}$ King Abdulaziz University, Jeddah, Saudi Arabia.

${ }^{7}$ School of Public Health, Ningxia Medical University, Yinchuan, P.R. China.

Published as: Youssef NA, Boswell E, Fiedler S, Jump R, Lee E, Yassa M, Khan F, Pathiraja A, Rizk $D$, Pearce M, O'Garo K, Currier JM, Koenig HG. Moral injury, posttraumatic stress disorder, and religious involvement among U.S. veterans. Ann Clin Psychiatry. 2018 May;30(2):113-121.

PubMed PMID: 29697712.

https://www.aacp.com/article/buy now/?id=528 


\section{$\underline{\text { Abstract }}$}

Background: Traumatic experiences can cause ethical conflicts. "Moral injury" (MI) has been used to describe this emotional/cognitive state, and may either contribute to the development of PTSD or block its recovery. We examine the relationship between MI and PTSD, and the impact of religious involvement (RI) on that relationship.

Methods: Study of 120 veterans enrolled at the Charlie Norwood VA Medical Center in Augusta, Georgia. Standard measures of PTSD symptoms, MI, and RI were administered. Regression models were utilized to examine correlates of PTSD symptoms and the moderating or mediating effects of RI.

Results: A strong relationship was found between MI and PTSD symptoms $(r=0.54, p<0.0001)$, and between $\mathrm{MI}$ and $\mathrm{RI}(r=-0.41, p<0.0001)$, but only a weak relationship was found between RI and PTSD symptoms $(r=-0.17, p=0.058)$. RI did not mediate or moderate the relationship between $\mathrm{MI}$ and PTSD symptoms in the overall sample. However, among non-Middle Eastern war theatre veterans, a significant buffering effect of religiosity was found.

Conclusions: MI has a strong positive relationship with PTSD symptoms and inverse relationship with RI. RI did not mediate or moderate the relationship between MI and PTSD in the overall sample, but moderated this relationship in non-Middle Eastern theatre veterans.

\section{Keywords}

Moral injury, post-traumatic stress disorder, spirituality, religiosity, veterans 


\section{INTRODUCTION}

Moral injury (MI) has been described in military personnel following traumatic experiences during wartime (Litz et al., 2009; Nash \& Litz, 2013; Shay, 1994). Litz and colleagues (2009) define it as "perpetrating, failing to prevent, bearing witness to, or learning about acts that transgress deeply held moral beliefs." MI, a construct that is distinct from PTSD, has often been identified in those with PTSD, and may contribute to the onset and maintenance of PTSD (Dokoupil; H. G. Koenig et al., 2017). While PTSD is believed to be a chronic emotional and physical response to fear and danger, with hyper-alertness and physiological arousal, which can be triggered by reminders of the trauma, MI does not necessarily involve fear-based stressors (Nieuwsma et al., 2015). Instead, MI results from experiences that violate deeply held moral beliefs and values, which then arouses shame, guilt, anger, and existential questioning. Furthermore, MI symptoms include not only psychological symptoms (guilt, anger, shame, etc.), but spiritual symptoms as well (loss of meaning and hope, spiritual struggles, and loss of faith) (Harold G Koenig et al., 2017). Treatments targeting MI, both spiritual and secular, have been shown to significantly reduce PTSD symptoms (Gray et al., 2012; Ogden et al., 2011).

Because of Ml's connection with moral beliefs and values, religious beliefs/practices may either worsen symptoms of this condition (e.g., due to high moral standards that are more easily violated) or may help to relieve $\mathrm{MI}$ (e.g., through beliefs regarding forgiveness, rituals involving forgiveness/penance, and support through the faith community) (Worthington \& Langberg, 2012). Religious beliefs and behaviors are important to many veterans, and are often used to cope with the stresses of war (H. G. Koenig et al., 1992; Harold G Koenig, Pargament, \& Nielsen, 1998). Regarding the relationship of religious belief/involvement and PTSD, it may be postulated that being more religious may have a protective effect on PTSD, on the other hand some suggested that such association may be that having mental health problems causes people to be less religious.

However, religious involvement (RI) has been shown in veterans to predict faster recovery from depression (H. G. Koenig et al., 1992), greater post-traumatic growth (Tsai, El-Gabalawy, Sledge, Southwick, \& Pietrzak, 2015), and faster recovery from PTSD (Currier, Holland, \& Drescher, 2015). On the other hand, religious struggles and loss of faith as a result of combat or combat-related experiences have been associated with lack of full recovery from PTSD and continued need for VA mental health services (Currier, Holland, \& Drescher, 2015; Fontana \& Rosenheck, 2004; Witvliet, Phipps, Feldman, \& Beckham, 2004). Ogden and colleagues (Ogden et al., 2011) also found that PTSD symptoms were associated with alienation from God, religious rifts, religious fear, and religious guilt among veterans of Middle Eastern wars. For the purpose of this study, Middle Eastern wars included OEF/OIF/OND veterans, that is to say the Middle Eastern region (Operation Iraqi Freedom, Operation New Dawn, and other Middle Eastern conflicts), as well as Afghanistan (Operation Enduring Freedom). Non-Middle Eastern wars included Vietnam, Korea, WWII era veterans, and miscellaneous other combat theatres.

MI may be particularly problematic for a certain subgroup of veterans, particularly younger veterans who served in Iraq/Afghanistan, and who may possibly experience more 
religious struggles (raised within a culture with high moral values, but perhaps fewer religious resources to cope with those moral struggles). Currier et al. (2014) compared communitydwelling adults of similar age and veterans who served in Iraq/Afghanistan and found less religiosity, more religious struggles, and weaker spirituality in the latter group. Thus, RI may either predispose veterans to the development of $\mathrm{MI}$ (and consequently worsen PTSD symptoms), may provide veterans with resources to help cope with and resolve $\mathrm{MI}$ (and consequently improve PTSD symptoms), or may buffer the effects of $\mathrm{MI}$ in generating and maintaining PTSD symptoms (blocking the negative effects of MI on PTSD). Further research is needed to better understand the potentially complex relationships and interactions between $\mathrm{RI}$, $\mathrm{MI}$, and PTSD symptoms.

\section{Objectives}

This study's objectives were to (1) examine the relationship between moral injury (MI) and severity of PTSD symptoms in U.S. military veterans, and (2) determine the mediating and moderating effects of religiosity on the relationship between $\mathrm{MI}$ and PTSD severity.

\section{METHODS}

This study was approved by the institutional review board (full review) and the Research and Development Committee of the Charlie Norwood VA Medical Center and by the Duke University Medical Center institutional review board (exempted). After informed consent, 120 veterans at the Charlie Norwood Veterans Administration Medical Center were recruited via advertisements and clinicians' referrals (from several mental health clinics including general mental health clinics and PTSD clinics) and completed a questionnaire that asked about demographics, military service information, physical health, use of alcohol, PTSD diagnosis, and information about religious affiliation, importance of religion and spirituality, and level of religious commitment. Inclusion criteria were being a veteran, deployed to a combat theater, and the presence of PTSD symptoms and/or symptoms of inner conflict. All participants gave written informed consent, and were compensated $\$ 25$ for their time in the form of a gift card.

\section{Questionnaire}

Demographics. Demographic information collected included age, gender, race (white, black, Hispanic, Asian, multiracial, other), education (years), and marital status (single, divorced, married, widowed).

Military Information. Participants were asked if they were involved in combat or served in a combat zone; the particular combat theatre that they were deployed to (Middle East, Vietnam, Korea/WWII, other); and the time since deployment (years). 
PTSD Symptoms/Diagnosis. Participants were also asked if they had PTSD symptoms without a formal diagnosis or had a formal PTSD diagnosis. PTSD severity was assessed with the 20-item PTSD Checklist of the Department of Veterans Affairs, DSM-5 version (PCL-5) (Weathers et al., 2013). The PCL-5 assesses the symptoms of PTSD as required for a diagnosis of PTSD in DSM-5. Participants were asked, "How much have you been bothered by each problem in the past month." Possible response options are 0 ("not at all"), 1 ("a little bit"), 2 ("moderately"), 3 ("quite a bit"), and 4 ("extremely"). Responses are summed to create a PTSD severity scale that ranges from 0 to 80 . The 20 -item PCL-5 has high reliability and strong associations with combat exposure, and functional impairment in military personnel (Ashbaugh, Houle-Johnson, Herbert, El-Hage, \& Brunet, 2016; Hoge, Riviere, Wilk, Herrell, \& Weathers, 2014).

Physical Health/Alcohol Use. Difficulty engaging in physical activity was assessed on a 0 to 10 scale ( $0=$ no difficulty, $10=$ great difficulty); similarly, average daily physical pain was assessed on a 0 to 10 scale ( $0=$ no pain, $10=$ severe pain). Finally, current alcohol use was inquired about, with possible responses ranging from "none" to "some" (2 drinks or less/day) to "quite a bit" (more than 2 drinks but less than 7 drinks per day) to "a lot" ( $>6$ drinks/day).

Religious Involvement. Religious affiliation was assessed as Christian, Non-Christian (of other religious persuasion), or none/atheist/agnostic. Importance of religion and importance of spirituality in life were each measured on a 4-point Likert scale from 1 (not important at all) to 4 (very important). Religious commitment and overall involvement were assessed with the 10-item Belief Into Action (BIAC) Scale (Harold G Koenig, Nelson, et al., 2015; Harold G Koenig, Wang, Al Zaben, \& Adi, 2015). The BIAC scale assesses degree of religious commitment, time spent in religious activity, and money given for religious causes, with responses ranging from 1 to 10 for each item. The internal reliability ( $\alpha=0.89,95 \% \mathrm{Cl}=0.86-0.91)$ and test-retest reliability for the BIAC after one week (ICC= $0.92,95 \% \mathrm{Cl}=0.87-0.95)$ are acceptable, and the scale has solid convergent, discriminant, and factor analytic validity (one factor explaining $94 \%$ of variance).

Moral Injury (MI). The MI Symptoms Scale is a 54-item multidimensional measure that assesses symptoms of moral injury, and is composed of 10 subscales: guilt, shame, violation of moral values, self-condemnation, betrayal by others, loss of trust, difficulty forgiving, loss of meaning, purpose and hope, spiritual struggles, and loss of faith (Harold G Koenig et al., 2017). We have recently shown in a multicenter study in veterans and active duty military the reliability and validity of this multi-dimensional symptom measure of moral injury that can be used in studies targeting $\mathrm{MI}$ in veterans and active duty military personnel with PTSD symptoms, and may also be used by clinicians to identify those at risk (H. G. Koenig et al., 2017). The guilt subscale consists of two items taken from the Moral Injury Questionnaire (MIQ) (Currier, Holland, Drescher, \& Foy, 2015) and three items from the Combat Guilt Scale (CGS) (Henning \& Frueh, 1997) (standardized Cronbach's alpha in the present sample $=0.41$ ). The shame subscale consists of two items from the CGS and an item from the work of Andrews with Veterans from the United Kingdom (Andrews, Brewin, Stewart, Philpott, \& Hejdenberg, 2009) (alpha=0.65). The 3-item violation of moral values subscale consists of statements of moral transgression from the Moral 
Injury Events Scale (MIES) (Nash \& Litz, 2013) that assess commission and omission (alpha=0.79). The self-condemnation subscale is made up of all 10 items of the Rosenberg Self-Esteem Scale (SES) (Rosenberg, 1965), which measures global self-worth by assessing both positive and negative feelings about the self (alpha=0.84). The 3 -item betrayal subscale from the MIES assesses feelings regarding betrayal by others during wartime (alpha $=0.62$ ). Six items make up the loss of trust subscale, and these come from the General Trust Scale (GTS) that assesses beliefs about the honesty and trustworthiness of others (Yamagishi \& Yamagishi, 1994) (alpha=0.80). Difficulty forgiving is measured by a 10 -item subscale consisting of six statements from the Heartland Forgiveness Scale (HFS) (Thompson et al., 2005) and four questions that ask about feeling forgiven by God, forgiving God, and forgiving self, and the need to seek forgiveness (alpha=0.69). Loss of meaning, purpose and hope is assessed by a 7-item subscale, with five items coming from the Presence of Meaning subscale of the Meaning in Life Questionnaire (MLQ) (Steger, Frazier, Oishi, \& Kaler, 2006), a sixth item coming from the MIQ, and a seventh item that asks how hopeful the individual is about the future (alpha=0.82). Finally, religious/spiritual struggle is assessed by the 7-item subscale of the Brief RCOPE (Pargament, Smith, Koenig, \& Perez, 1998) and loss of faith subscale by a single question that asks how faith has changed as a result of wartime experiences (alpha $=0.87$ ).

All 54 items of the MI Symptom Scale are rated on a 1-10 scale in terms of agreement or disagreement to each statement (with reverse scoring of items so that higher scores indicate more symptoms of $\mathrm{MI}$ ) producing a response ranging from 54 to 540 . The average $\mathrm{MI}$ score in the present sample was 262.0 with a SD of 68.0 and range from 119 to 452 . Overall, the MI Symptom Scale had an alpha of 0.92 .

Missing Values. In constructing scales, missing items on the scales were handled as follows. If participants answered at least $50 \%$ of items on a scale or subscale, the average of items answered was substituted for the missing item score (done for the PCL-5 in $12 \%$ of cases, the $\mathrm{BIAC}$ in $3 \%$, and the $\mathrm{MI}$ subscales in $0 \%$ to $13 \%$ ). A similar procedure was followed for combining subscale scores to produce the overall MI Symptom Scale score. If an entire subscale score was missing, the average of completed subscale scores was inserted for the missing score (done in $7 \%$ of cases).

\section{Statistical Analyses}

Descriptive statistics were used to report characteristics of the sample with means (and standard deviations) and frequency distributions. Bivariate analyses were conducted using Pearson's $r$ for continuous variables, Student's t-test for continuous variables across dichotomized categories, analysis of variance (ANOVA) for continuous variables across three or more categories, and chi-square for two categorical variables. Variables significant at $p<0.15$ in bivariate analyses were entered into general linear models predicting PTSD symptom severity that consisted of the MI Symptom Scale score alone (Model 1), followed by adding demographic 
and military variables (Model 2), then physical health and behavioral variables (Model 3), then religious commitment (BIAC) (Model 4), and then the interaction between $\mathrm{MI}$ symptoms and BIAC score (to test the moderating effect of religiosity on the relationship between MI and PTSD symptom severity). For Models 4 and 5, the BIAC was dichotomized with two-thirds of the sample in the low religiosity category and one-third in the high category. Alpha level was set at $p=0.05$, and was not corrected for multiple comparisons due to the exploratory nature of these analyses. All statistical tests were performed using SAS (version 9.4; SAS Institute Inc., Cary, NC).

\section{RESULTS}

Sample characteristics are displayed in Table 1. The average age of participants was 55.6 years with a range from 23 to 81 years. The majority of participants were male $(86.4 \%)$, AfricanAmerican (62.9\%) or white Caucasian (30.2\%), married (43.7\%) or divorced (29.4\%), and Christian (84.9\%). Average education was 13.8 years with a range of 4 to 25 years. This sample contained veterans who were deployed. The majority were involved in combat $(67.5 \%)$, served in Iraq, Afghanistan, or other Middle Eastern theatres (48.7\%) or Vietnam (31.3\%), and the average time since last deployment was 23.2 years (range 6 months to 60 years). The vast majority indicated they had a formal diagnosis of PTSD (93.1\%), and the average PCL-5 score was 53.7 (where the cut-off for PTSD ranges from 28 to 38) (Ashbaugh et al., 2016). Most participants experienced some level of physical disability (average 6.1 on a 0 to 10 scale) and daily chronic pain (average 6.5 on a 0 to 10 scale). Religion and spirituality were "very important" to many $(54.2 \%$ and $59.3 \%$, respectively), and the average BIAC score was 47.1 (SD 20.9), similar to the average score found in stressed family caregivers of disabled persons in North Carolina (47.2, SD=21.5) (Harold G Koenig, Wang, et al., 2015).

\section{Bivariate Correlations}

As hypothesized, $\mathrm{Ml}$ symptoms were strongly and positively correlated with PTSD symptom severity $(r=0.54, p<0.0001)$ (Table 2$)$. In contrast, $\mathrm{Ml}$ symptoms were strongly and inversely correlated with religious involvement assessed by the BIAC $(r=-0.41, p<0.0001)$. However, religiosity was only weakly correlated with PTSD symptom severity $(r=-0.17, p=0.058)$. Other significant correlates of PTSD symptoms were higher education level, deployment to Afghanistan/Iraq or other Middle Eastern war theatres, and especially impairments in physical functioning $(r=0.40, p<0.0001)$ or daily chronic physical pain $(r=0.27, p<0.005)$. Compared to veterans serving in other combat theatres, $\mathrm{MI}$ and PTSD severity were both higher in veterans serving in the Middle East ( $F=6.9, p=0.002$ and $F=2.4, p<0.10$, respectively), whereas religiosity (BIAC) was significantly lower $(F=5.36, p=0.006)$. 


\section{Multivariate Analyses}

The strongest independent predictor of PTSD symptom severity was MI symptoms (Table 3). Higher education and being married (surprisingly) were other significant independent predictors of PTSD symptom severity. Poorer physical functioning was also a significant predictor. Once $\mathrm{MI}$ symptoms were controlled for, religiosity score on the BIAC (either continuous or dichotomized) was no longer related to PTSD symptoms and did not mediate the relationship between $\mathrm{MI}$ symptoms and PTSD symptoms in the overall sample. Furthermore, the interaction between $\mathrm{MI}$ and BIAC scores was not significant, indicating no buffering effect for religiosity on the relationship between $\mathrm{MI}$ and PTSD symptom severity.

Given that both MI and PTSD symptoms were higher and religiosity lower in Middle East theatre veterans, further exploratory analyses were conducted. The buffering effect of religiosity on the relationship between MI and PTSD symptoms was examined separately in Middle Eastern theatre veterans and non-Middle Eastern theatre veterans (Vietnam, Korea, WWII, and other theatres). Given the small samples in these sub-analyses, only MI, BIAC score (continuous), and the interaction between $\mathrm{MI}$ and BIAC scores were examined as predictors of PTSD severity in these models. Among non-Middle Eastern theatre veterans ( $N=63$, including 3 missing data for combat theatre), a significant interaction was found between $\mathrm{MI}$ and $\mathrm{BIAC}$ scores $(\mathrm{B}=-0.004$, $\mathrm{SE}=0.002, \mathrm{t}=-2.09, \mathrm{p}=0.04)$, whereas this was not found in Middle Eastern theatre veterans $(\mathrm{N}=56)$ $(B=0.001, S E=0.001, t=0.80, p=0.43)$. To determine what the significant interaction meant in nonMiddle Eastern theatre veterans, we again dichotomized veterans in terms of high and low religiosity. Among highly religious non-Middle Eastern theatre veterans ( $N=31)$, no significant correlation was found between $\mathrm{MI}$ and PTSD symptom severity $(B=0.04, S E=0.05, t=0.68, p=0.50)$, whereas among less religious non-Middle Eastern theatre veterans ( $N=32)$, a significant positive correlation between $\mathrm{MI}$ and PTSD symptoms was present $(B=0.14, S E=0.04, t=3.19, p=0.003)$.

\section{DISCUSSION}

To our knowledge, this is the first study to explore the role of religious involvement in the established relationship between symptoms of moral injury resulting from wartime experiences and PTSD symptom severity. In the overall sample, we found that MI was strongly and positively correlated with severity of PTSD symptoms, although level of religious involvement or commitment had little or no effect on this relationship in the overall sample, neither mediating nor buffering it. However, in an exploratory analysis among non-Middle Eastern theatre veterans, who were typically older and more religious, high religiosity completely buffered the effects of MI on PTSD symptoms. However, there was no evidence for such a buffering effect among younger veterans who were deployed in recent operations in Iraq, Afghanistan, and other Middle East conflicts. 
Other researchers have likewise reported a strong correlation between exposure to morally injurious events and PTSD symptoms or another psychopathology (A. O. Bryan, Bryan, Morrow, Etienne, \& Ray-Sannerud, 2014; C. J. Bryan et al., 2016; Currier, Holland, Drescher, et al., 2015; Nash et al., 2013; Nickerson et al., 2015; Wisco et al., 2017). However, moral injury scales used to examine these correlations assessed a mixture of traumatic experiences (not amenable to treatment) and emotional symptoms (treatable), and they did not assess spiritual symptoms of moral injury (e.g., religious struggles or loss of faith). While no studies (to our knowledge) have examined the buffering effect of religiosity on the relationship between $\mathrm{MI}$ and PTSD symptoms, several studies with larger samples of veterans have similarly found a significant inverse correlation between religious involvement and PTSD symptoms (Currier, Drescher, \& Harris, 2014; Currier, Holland, \& Drescher, 2015; Hourani et al., 2012; Sharma et al., 2017). Likewise, religiosity has also been shown to buffer the effects of traumatic experiences on mental health outcomes in veterans and non-veterans (Bentley, Ahmad, \& Thoburn, 2014; Chang, Skinner, \& Boehmer, 2001; Chang, Skinner, Zhou, \& Kazis, 2003; Korn \& Zukerman, 2011).

While the present study found a weak inverse relationship between religiosity and PTSD symptom severity in bivariate analyses, this relationship lost its borderline significance when other factors were controlled for in the multivariate analyses. Although religiosity was inversely related to $\mathrm{MI}$, it did not buffer the effects of $\mathrm{MI}$ on PTSD symptoms in the overall sample. However, religiosity buffered the effects of MI on PTSD symptoms among non-Middle Eastern theatre veterans. Thus, it appeared that religiosity among veterans of Vietnam, WWII, Korea, and other non-Middle Eastern theatres helped to block the effects of MI on PTSD symptoms or to promote recovery when such difficulties emerged, whereas religiosity had little effect on this relationship among veterans serving in Iraq, Afghanistan, or other Middle Eastern theatres. The latter group also tended to be less religious, yet have higher MI and PTSD symptom scores. Although difficult to explain, perhaps religiosity was less effective in helping Middle Eastern theatre veterans cope with the moral injuries that they had suffered, thus inducing or increasing the severity of their PTSD symptoms.

These findings, if verified in future studies, have clinical implications. MI involves not only psychological symptoms such as guilt and shame, but also spiritual symptoms such as religious struggles and loss of religious faith. Neither symptom type can be ignored in any comprehensive treatment for those with PTSD. Psychotherapies, both spiritually-integrated and secular forms, are needed to target the psychological and the spiritual symptoms of moral injury or inner ethical conflict. Based on the present findings, spiritual therapies for MI in the setting of PTSD may be particularly effective in older veterans who have served in non-Middle Eastern war theatres. The limitations of the present study, however, make such conclusions preliminary.

\section{Limitations}


Several study characteristics limit the generalizability and interpretability of these findings. First, the cross-sectional nature prevents any speculations on direction of causation in the relationships identified. Second, this was a relatively small sample and one that was drawn from a single state (Georgia), one located in the Bible Belt (although many of these veterans were not originally from Georgia). Third, there may be some overlap between our measure of religious involvement (BIAC) and the spiritual dimensions of moral injury (MI), which needs to be acknowledged; however, these dimensions are quite distinct since spiritual struggles may occur in those who are religious or in those who are not religious, and often there is little relationship between the two (as has been found for positive and negative religious coping). Finally, significant buffering effects of religiosity were identified only in exploratory analyses, and significance levels were not corrected for multiple comparisons. However, the strengths of this study are the use of psychometrically valid comprehensive measures of MI symptoms (symptoms that are amenable to change), PTSD symptom severity, and religious commitment, along with careful statistical control for demographic, military, and physical health confounders.

\section{CONCLUSIONS}

While symptoms of moral injury appeared to be strongly and positively correlated with severity of PTSD symptoms (the strongest of any correlates identified here), level of religious involvement in the overall sample did not influence this relationship--neither mediating nor buffering it. However, among veterans serving in non-Middle Eastern combat zones, religiosity appeared to buffer the effects of moral injury on PTSD symptom severity. Future studies are needed that longitudinally follow active-duty military from prior to deployment to discharge from the service, prospectively examining the effects of baseline religiosity on MI and PTSD symptoms, taking into account the severity of wartime trauma and confounding factors such as pre-service mental health and physical injuries experienced during combat. Randomized clinical trials are also needed to determine whether interventions that specifically target MI in those with PTSD result in a faster remission of PTSD symptoms and its comorbidities compared to standard therapies used today. 
Acknowledgments: We thank the veterans who participated in this study, and all the staff at Charlie Norwood VA who assisted with this study especially Dr. Thomas Hartney and Dr. Yekeen Aderibigbe. We thank Walid Farkouh for his assistance in some of the IRB submission.

Funding: This study was supported by a grant from the Augusta Biomedical Research institute to Nagy Youssef. 
TABLE 1

Characteristics of the sample $(\mathrm{N}=120)$

\begin{tabular}{|c|c|}
\hline & $\%$ (n) / Mean (SD) (range) \\
\hline Age, years & $55.6(14.8)(23$ to 81$)$ \\
\hline Sex (\% female) & $13.6 \%(16)$ \\
\hline Race (\% black) & $62.9 \%(73)$ \\
\hline Education, years & $13.8(3.2)(4$ to 25$)$ \\
\hline Marital status (\% married) & $43.7 \%(52)$ \\
\hline Involved in combat (\% yes) & $67.5 \%(79)$ \\
\hline \multicolumn{2}{|l|}{ Combat theater } \\
\hline Middle Eastern & $48.7 \%(56)$ \\
\hline Vietnam & $31.0 \%(36)$ \\
\hline Other & $20.7 \%(23)$ \\
\hline Time since deployment, years & $24.2(17.3)(0.5$ to 60$)$ \\
\hline Diagnosis of PTSD (\% yes) & $93.1(108)$ \\
\hline $\begin{array}{l}\text { Physical functioning, average } \\
\text { on } 0 \text { to } 10 \text { scale }\end{array}$ & $6.1(2.6)(0$ to 10$)$ \\
\hline $\begin{array}{l}\text { Pain severity, average on } \\
0 \text { to } 10 \text { scale }\end{array}$ & $6.5(2.4)(0$ to 10$)$ \\
\hline Alcohol use ( $\%$ > 2 drinks/day) & $13.8 \%(16)$ \\
\hline \multicolumn{2}{|l|}{ Religious affiliation } \\
\hline Christian & $84.9 \%(101)$ \\
\hline Non-Christian & $2.5 \%(2)$ \\
\hline None, agnostic, atheist & $12.6 \%(15)$ \\
\hline Importance of religion (\% very) & $54.2 \%(65)$ \\
\hline Importance of spirituality (\% very) & $59.3 \%(70)$ \\
\hline Belief into Action Scale score & 47.1 (20.9) (10 to 93$)$ \\
\hline PCL-5 score & 53.7 (15.3) (15 to 80$)$ \\
\hline Ml symptom score & $262(68.0)$ (119 to 452$)$ \\
\hline
\end{tabular}

$\mathrm{N}$ may vary by up to $5 \%$.

MI: moral injury; PCL-5: PTSD Symptom Checklist DSM-5 version;

PTSD: posttraumatic stress disorder; SD: standard deviation. 
TABLE 2

Sample characteristics and PTSD symptoms (PCL-5) (bivariate correlations)

\begin{tabular}{|c|c|c|c|}
\hline Demographic & $\boldsymbol{r}$ & $\begin{array}{c}\text { PCL-5 } \\
\text { (mean, SD) }\end{array}$ & $\boldsymbol{P}$ \\
\hline Age, years & -.11 & & .23 \\
\hline Education, years & .26 & & $<01$ \\
\hline \multicolumn{4}{|l|}{ Race } \\
\hline Black & & $54.0(14.2)$ & .96 \\
\hline Other & & $54.2(16.9)$ & \\
\hline \multicolumn{4}{|l|}{ Marital status } \\
\hline Married & & $55.9(13.2)$ & .14 \\
\hline Other & & $51.7(16.8)$ & \\
\hline \multicolumn{4}{|l|}{ Military } \\
\hline \multicolumn{4}{|l|}{ Involved in combat } \\
\hline Yes & & $54.7(15.2)$ & .35 \\
\hline No & & $51.8(16.0)$ & \\
\hline \multicolumn{4}{|l|}{ Combat theater } \\
\hline Middle Eastern & & $56.6(14.8)$ & .04 \\
\hline Other & & $50.9(15.4)$ & \\
\hline Years since deployed & -.10 & & .30 \\
\hline \multicolumn{4}{|l|}{ Diagnosis of PTSD } \\
\hline Yes & & $54.7(14.9)$ & .02 \\
\hline No & & $40.7(19.6)$ & \\
\hline
\end{tabular}

\section{Physical health/health behaviors}

\begin{tabular}{|c|c|c|c|}
\hline $\begin{array}{l}\text { Physical functioning } \\
(0 \text { to } 10)\end{array}$ & .40 & & $<.0001$ \\
\hline Pain severity (0 to 10 ) & .27 & & $<.005$ \\
\hline \multicolumn{4}{|l|}{ Alcohol use } \\
\hline$>2$ drinks/day & & $58.8(16.8)$ & .15 \\
\hline$<3$ drinks/day & & $52.8(15.2)$ & \\
\hline \multicolumn{4}{|l|}{ Religious } \\
\hline \multicolumn{4}{|l|}{ Christian } \\
\hline Yes & & $52.9(15.2)$ & .19 \\
\hline No & & $58.1(15.6)$ & \\
\hline \multicolumn{4}{|l|}{ Religious importance } \\
\hline Very important & & $53.1(14.9)$ & .66 \\
\hline More than very & & $54.4(15.8)$ & \\
\hline \multicolumn{4}{|l|}{ Spiritual importance } \\
\hline Very important & & $52.4(14.8)$ & .31 \\
\hline More than very & & $55.4(16.2)$ & \\
\hline BIAC score (religiosity) & -.17 & & .058 \\
\hline MI Symptom score & .54 & & $<.0001$ \\
\hline
\end{tabular}

BIAC: Bellef Into Action Scale; MI: moral Injury, PCL-5: PTSD Checidlst DSM-5 version; SD: standard devation. 
TABLE 3

Predictors of PTSD severity (PCL-5) (multivariate)

\begin{tabular}{|c|c|c|c|c|c|}
\hline & $\begin{array}{c}\text { Model } 1 \\
\text { beta (SE) }\end{array}$ & $\begin{array}{c}\text { Model } 2 \\
\text { beta (SE) }\end{array}$ & $\begin{array}{c}\text { Model } 3 \\
\text { beta (SE) }\end{array}$ & $\begin{array}{c}\text { Model } 4 \\
\text { beta (SE) }\end{array}$ & $\begin{array}{l}\text { Model } 5 \\
\text { beta (SE) }\end{array}$ \\
\hline $\mathrm{Ml}$ & $.12(.02)^{\mathrm{a}}$ & $.13(.02)^{\mathrm{a}}$ & $.12(.02)^{\mathrm{a}}$ & $.12(.02)^{\mathrm{a}}$ & $.12(.02)^{\mathrm{a}}$ \\
\hline \multicolumn{6}{|l|}{ Demographic and military } \\
\hline Education, years & $\ldots$ & $1.17(.36)^{\mathrm{b}}$ & $1.13(.36)^{\mathrm{c}}$ & $1.14(.36)^{\mathrm{c}}$ & $1.13(.36)^{\mathrm{c}}$ \\
\hline Marital status (married) & $\ldots$ & $6.56(2.33)^{\mathrm{c}}$ & $5.62(2.40)^{d}$ & $5.69(2.41)^{d}$ & $5.69(2.43)^{d}$ \\
\hline Combat theater (Middle Eastern) & $\ldots$ & $-.37(2.42)$ & $-.95(2.49)$ & $-1.68(2.68)$ & $-1.69(2.69)$ \\
\hline \multicolumn{6}{|l|}{ Physical health/behaviors } \\
\hline Physical functioning (0 to 10 ) & $\ldots$ & $\ldots$ & $1.07(.54)^{d}$ & $1.11(.55)^{d}$ & $1.10(.55)^{\mathrm{d}}$ \\
\hline Pain severity (0 to 10) & $\ldots$ & $\ldots$ & $.28(.57)$ & $.34(.58)$ & $.34(.58)$ \\
\hline Alcohol use (>2 drinks/day) & $\ldots$ & $\ldots$ & $.87(3.49)$ & $.70(3.51)$ & $.77(3.55)$ \\
\hline \multicolumn{6}{|l|}{ Religious } \\
\hline BIAC score (dichotomized) & $\ldots$ & $\ldots$ & $\ldots$ & $-2.10(2.81)$ & $-.30(10.27)$ \\
\hline Interaction (MI × BIAC) & $\ldots$ & $\ldots$ & $\ldots$ & $\ldots$ & $-.01(.04)$ \\
\hline Model R-square (n) & $.29(120)^{a}$ & $.42(110)^{\mathrm{a}}$ & $.46(107)^{\mathrm{a}}$ & $.47(107)^{\mathrm{a}}$ & $.47(107)^{\mathrm{a}}$ \\
\hline
\end{tabular}

Variables included in multivariate model only if $\mathrm{P}<.15$.

BIAC: Belief into Action Scale; MI: moral injury; PCL-5: PTSD Checklist DSM-5 version; PTSD: posttraumatic stress disorder; SE: standard error.

aP $\leq .0001$.

$\mathrm{b} P \leq .001$.

$c P \leq .01$.

$\mathrm{d} P \leq .05$. 
Contributions (added for the purpose of this thesis): Nagy Youssef was the study PI and the study psychiatrist at the Charlie Norwood VA Medical Center. Youssef obtained a grant from the Augusta Biomedical Research Foundation for funding the study. He supervised the research staff and supervised all aspects of the study implementation. 


\section{REFERENCES}

Andrews, B., Brewin, C. R., Stewart, L., Philpott, R., \& Hejdenberg, J. (2009). Comparison of immediate-onset and delayed-onset posttraumatic stress disorder in military veterans. $J$ Abnorm Psychol, 118(4), 767-777. doi:10.1037/a0017203

Ashbaugh, A. R., Houle-Johnson, S., Herbert, C., El-Hage, W., \& Brunet, A. (2016). Psychometric validation of the English and French versions of the Posttraumatic Stress Disorder Checklist for DSM-5 (PCL-5). PloS one, 11(10), e0161645.

doi:10.1371/journal.pone.0161645

Bentley, J., Ahmad, Z., \& Thoburn, J. (2014). Religiosity and posttraumatic stress in a sample of East African refugees. Mental Health, Religion \& Culture, 17(2), 185-195. doi:10.1080/13674676.2013.784899

Bryan, A. O., Bryan, C. J., Morrow, C. E., Etienne, N., \& Ray-Sannerud, B. (2014). Moral injury, suicidal ideation, and suicide attempts in a military sample. Traumatology, 20(3), 154160. doi:10.1037/h0099852

Bryan, C. J., Bryan, A. O., Anestis, M. D., Anestis, J. C., Green, B. A., Etienne, N., . . RaySannerud, B. (2016). Measuring moral injury: psychometric properties of the Moral Injury Events Scale in two military samples. Assessment, 23(5), 557-570. doi:10.1177/1073191115590855

Chang, B. H., Skinner, K. M., \& Boehmer, U. (2001). Religion and mental health among women veterans with sexual assault experience. Int J Psychiatry Med, 31(1), 77-95. doi:10.2190/ONQA-YAJ9-WOAM-YB3P

Chang, B. H., Skinner, K. M., Zhou, C., \& Kazis, L. E. (2003). The relationship between sexual assault, religiosity, and mental health among male veterans. Int J Psychiatry Med, 33(3), 223-239. doi:10.2190/NM3D-EWYR-4B59-DFM8

Currier, J. M., Drescher, K., \& Harris, J. I. (2014). Spiritual functioning among veterans seeking residential treatment for PTSD: a matched control group study. Spirituality in Clinical Practice, 1(1), 3-15. doi:10.1037/scp0000004

Currier, J. M., Holland, J. M., Drescher, K., \& Foy, D. (2015). Initial psychometric evaluation of the Moral Injury Questionnaire--Military version. Clin Psychol Psychother, 22(1), 54-63. doi:10.1002/cpp.1866

Currier, J. M., Holland, J. M., \& Drescher, K. D. (2015). Spirituality factors in the prediction of outcomes of PTSD treatment for U.S. military veterans. J Trauma Stress, 28(1), 57-64. doi:10.1002/jts.21978

Dokoupil, T. (3 Dec 2012). A new theory of PTSD and veterans: moral injury. Newsweek. Retrieved from https://www.newsweek.com/new-theory-ptsd-and-veterans-moralinjury-63539 
Fontana, A., \& Rosenheck, R. (2004). Trauma, change in strength of religious faith, and mental health service use among veterans treated for PTSD. J Nerv Ment Dis, 192(9), 579-584. doi:10.1097/01.nmd.0000138224.17375.55

Gray, M. J., Schorr, Y., Nash, W., Lebowitz, L., Amidon, A., Lansing, A., . . Litz, B. T. (2012). Adaptive disclosure: an open trial of a novel exposure-based intervention for service members with combat-related psychological stress injuries. Behav Ther, 43(2), 407-415. doi:10.1016/j.beth.2011.09.001

Henning, K. R., \& Frueh, B. C. (1997). Combat guilt and its relationship to PTSD symptoms. J Clin Psychol, 53(8), 801-808. doi:10.1002/(SICI)1097-4679(199712)53:8<801::AIDJCLP3>3.0.CO;2-1

Hoge, C. W., Riviere, L. A., Wilk, J. E., Herrell, R. K., \& Weathers, F. W. (2014). The prevalence of post-traumatic stress disorder (PTSD) in US combat soldiers: a head-to-head comparison of DSM-5 versus DSM-IV-TR symptom criteria with the PTSD checklist. Lancet Psychiatry, 1(4), 269-277. doi:10.1016/S2215-0366(14)70235-4

Hourani, L. L., Williams, J., Forman-Hoffman, V., Lane, M. E., Weimer, B., \& Bray, R. M. (2012). Influence of spirituality on depression, posttraumatic stress disorder, and suicidality in active duty military personnel. Depress Res Treat, 2012, 425463. doi:10.1155/2012/425463

Koenig, H. G., Boucher, N. A., Oliver, R. J. P., Youssef, N., Mooney, S. R., Currier, J. M., \& Pearce, M. (2017). Rationale for spiritually oriented cognitive processing therapy for moral injury in active duty military and veterans with posttraumatic stress disorder. Journal of Nervous and Mental Disease, 205(2), 147-153. doi:10.1097/NMD.0000000000000554

Koenig, H. G., Cohen, H. J., Blazer, D. G., Pieper, C., Meador, K. G., Shelp, F., . . . DiPasquale, B. (1992). Religious coping and depression among elderly, hospitalized medically ill men. Am J Psychiatry, 149(12), 1693-1700. doi:10.1176/ajp.149.12.1693

Koenig, H. G., Nelson, B., Shaw, S. F., Al Zaben, F., Wang, Z., \& Saxena, S. (2015). Belief into Action scale: a brief but comprehensive measure of religious commitment. Open Journal of Psychiatry, 5(1), 66-77. doi:10.4236/ojpsych.2015.51010

Koenig, H. G., Oliver, J. P., Ames, D., Erickson, Z., Youssef, N. A., Haynes, K., . . Currier, J. M. (2017). The inner conflict moral injury symptom scale. Durham, NC: Duke University Center for Spirituality, Theology, and Health.

Koenig, H. G., Pargament, K. I., \& Nielsen, J. (1998). Religious coping and health status in medically ill hospitalized older adults. Journal of Nervous and Mental Disease, 186(9), 513-521.

Koenig, H. G., Wang, Z., Al Zaben, F., \& Adi, A. (2015). Belief into Action Scale: a comprehensive and sensitive measure of religious involvement. Religions, 6(3), 1006-1016. doi:10.3390/rel6031006

Korn, L., \& Zukerman, G. (2011). Affective and behavioral changes following exposure to traumatic events: the moderating effect of religiosity on avoidance behavior among 
students studying under a high level of terror event exposure. J Relig Health, 50(4), 911921. doi:10.1007/s10943-011-9502-8

Litz, B. T., Stein, N., Delaney, E., Lebowitz, L., Nash, W. P., Silva, C., \& Maguen, S. (2009). Moral injury and moral repair in war veterans: a preliminary model and intervention strategy. Clin Psychol Rev, 29(8), 695-706. doi:10.1016/j.cpr.2009.07.003

Nash, W. P., \& Litz, B. T. (2013). Moral injury: a mechanism for war-related psychological trauma in military family members. Clin Child Fam Psychol Rev, 16(4), 365-375. doi:10.1007/s10567-013-0146-y

Nash, W. P., Marino Carper, T. L., Mills, M. A., Au, T., Goldsmith, A., \& Litz, B. T. (2013). Psychometric evaluation of the Moral Injury Events Scale. Mil Med, 178(6), 646-652. doi:10.7205/MILMED-D-13-00017

Nickerson, A., Schnyder, U., Bryant, R. A., Schick, M., Mueller, J., \& Morina, N. (2015). Moral injury in traumatized refugees. Psychother Psychosom, 84(2), 122-123. doi:10.1159/000369353

Nieuwsma, J. A., Walser, R. D., Farnsworth, J. K., Drescher, K. D., Meador, K. G., \& Nash, W. (2015). Possibilities within acceptance and commitment therapy for approaching moral injury. Current Psychiatry Reviews, 11(3), 193-206. doi:10.2174/1573400511666150629105234

Ogden, H., Harris, J. I., Erbes, C. R., Engdahl, B. E., Olson, R. H., Winskowski, A. M., \& McMahill, J. (2011). Religious functioning and trauma outcomes among combat veterans. Counseling et spiritualité, 30(1), 71-89.

Pargament, K. I., Smith, B. W., Koenig, H. G., \& Perez, L. (1998). Patterns of positive and negative religious coping with major life stressors. Journal for the scientific study of religion, 37(4), 710-724. doi:10.2307/1388152

Rosenberg, M. (1965). Society and the adolescent self-image. Princeton, NJ: Princeton University Press.

Sharma, V., Marin, D. B., Koenig, H. K., Feder, A., lacoviello, B. M., Southwick, S. M., \& Pietrzak, R. H. (2017). Religion, spirituality, and mental health of U.S. military veterans: results from the National Health and Resilience in Veterans Study. J Affect Disord, 217, 197-204. doi:10.1016/j.jad.2017.03.071

Shay, J. (1994). Achilles in Vietnam: combat trauma and the undoing of character. New York: Atheneum.

Steger, M. F., Frazier, P., Oishi, S., \& Kaler, M. (2006). The meaning in life questionnaire: assessing the presence of and search for meaning in life. Journal of counseling psychology, 53(1), 80-93. doi:10.1037/0022-0167.53.1.80

Thompson, L. Y., Snyder, C. R., Hoffman, L., Michael, S. T., Rasmussen, H. N., Billings, L. S., . . . Roberts, D. E. (2005). Dispositional forgiveness of self, others, and situations. J Pers, 73(2), 313-359. doi:10.1111/j.1467-6494.2005.00311.x 
Tsai, J., El-Gabalawy, R., Sledge, W. H., Southwick, S. M., \& Pietrzak, R. H. (2015). Post-traumatic growth among veterans in the USA: results from the National Health and Resilience in Veterans Study. Psychol Med, 45(1), 165-179. doi:10.1017/S0033291714001202

Weathers, F. W., Litz, B. T., Keane, T. M., Palmieri, P. A., Marx, B. P., \& Schnurr, P. P. (2013). The PTSD Checklist for DSM-5 (PCL-5). Retrieved from National Center for PTSD: https://www.ptsd.va.gov/professional/assessment/adult-sr/ptsd-checklist.asp\#obtain

Wisco, B. E., Marx, B. P., May, C. L., Martini, B., Krystal, J. H., Southwick, S. M., \& Pietrzak, R. H. (2017). Moral injury in U.S. combat veterans: results from the National Health and Resilience in Veterans Study. Depress Anxiety, 34(4), 340-347. doi:10.1002/da.22614

Witvliet, C. V., Phipps, K. A., Feldman, M. E., \& Beckham, J. C. (2004). Posttraumatic mental and physical health correlates of forgiveness and religious coping in military veterans. $J$ Trauma Stress, 17(3), 269-273. doi:10.1023/B:JOTS.0000029270.47848.e5

Worthington, E. L., \& Langberg, D. (2012). Religious considerations and self-forgiveness in treating complex trauma and moral injury in present and former soldiers. Journal of Psychology \& Theology, 40(4), 274-280. doi:10.1177/009164711204000403

Yamagishi, T., \& Yamagishi, M. (1994). Trust and commitment in the United States and Japan. Motivation and emotion, 18(2), 129-166. doi:10.1007/BF02249397 



\section{Chapter 8 Relapse Prevention After Index Electroconvulsive Therapy in Treatment-Resistant Depression}

Nagy A. Youssef, MD ${ }^{1,2}$, W. Vaughn McCall, MD, MS ${ }^{1}$

1 Department of Psychiatry \& Health Behavior, Medical College of Georgia at Augusta University, Augusta, GA 30912 USA

2 Academic Affairs, Medical College of Georgia, Augusta University, 30912 Augusta, GA 30912 USA

Published as: Youssef NA, McCall WV. Relapse prevention after index electroconvulsive therapy in treatment-resistant depression. Ann Clin Psychiatry. 2014 Nov;26(4):288-96. Review. PubMed PMID: 25401716; PubMed Central PMCID: PMC4420179. https://www.aacp.com/article/buy now/?id=120 


\section{Abstract}

Background: One-third of patients who suffer from depression are resistant to conventional treatments. An acute course of electroconvulsive therapy (ECT) can lead to remission of depressive symptoms in a substantial portion of the treatment-resistant patients. However, prevention of relapse with depressive symptoms after an index course of ECT can be challenging. We reviewed pertinent studies on the topic and analyzed the best strategies to avoid relapse and recurrence of depressive symptoms.

Methods: We performed a systematic literature review of PubMed through April 2014 for clinical trials published in English to determine if continuation ECT, continuation medication, continuation psychotherapy, or combinations of these are the best strategy to avoid relapse and recurrence of depressive symptoms after an acute course of ECT. Clinical trials comparing $\geq 2$ of the above strategies were included in the review.

Results: Although there are few rigorous randomized clinical trials in this area, most studies suggest that combined continuation ECT (C-ECT) and continuation pharmacotherapy are the most effective strategy in relapse prevention.

Conclusions: C-ECT and continuation pharmacotherapy may be more effective than either alone for preventing relapse. However, more definitive randomized clinical trials are needed. 


\section{INTRODUCTION}

Approximately 20.9 million American adults a year suffer from a mood disorder (Kessler, Chiu, Demler, Merikangas, \& Walters, 2005). Major depressive disorder (MDD) is the most prevalent mood disorder, and is the leading cause of disability among Americans age 15 to 44 (World Health Organization, 2008). It also is a risk factor for mortality including death due to suicide and other medical conditions. One-third of patients do not respond to pharmacotherapy (including medication combinations) and psychotherapy (Rush et al., 2006). Among treatmentresistant patients, $>100,000$ patients per year in the United States are treated with electroconvulsive therapy (ECT)(Dukakis \& Tye, 2007); relapse after ECT is common (Coppen et al., 1981), however, and strategies for reducing the risk of relapse after ECT are under-studied. This review will focus on prevention of relapse or recurrence of depressive episodes of mood disorders after a successful acute course of ECT (depressive episodes being the most common indication for ECT).

Psychotherapeutic interventions such as cognitive-behavioral therapy can help patients with mild to moderate depressive disorders and should be tried early in treatment. Psychopharmacological agents are indicated for severe depression (Davidson, 2010). Unfortunately, many patients do not respond or experience remission while taking psychopharmacological agents. For example, the response rate to a first antidepressant is $50 \%$, and remission is only $37 \%$.

\section{Definition of response, remission, and relapse in ECT trials}

In antidepressant trials, treatment response often is defined as a reduction in Hamilton Rating Scale for Depression (HRSD) Score of $\geq 50 \%$ (Frank et al., 1991). In psychotherapy trials, treatment response often is defined similarly to pharmacotherapy trials (Driessen et al., 2013). However, in ECT clinical trials, response often is defined as reduction in HRSD-24 of $\geq 60 \%$ after an ECT course. The definition of remission in many ECT trials is symptom reduction of $\geq 60 \%$ on HRSD scores and a final HRSD score $\leq 10$ (Petrides et al., 2001; Sackeim et al., 2001; Sackeim et al., 2000).

Some studies (Prudic, Olfson, Marcus, Fuller, \& Sackeim, 2004) had 2 definitions of remission: "moderate and strict criteria." In Prudic et al., the "moderate criteria (remitter10)" is a reduction of $\geq 60 \%$ on HRSD scores and a final HRSD score of $\leq 10$. The "strict criteria (remitter7)" is a reduction of $\geq 60 \%$ on HRSD scores and a final HRSD score of $\leq 7$ (Prudic et al., 2004).

Relapse in clinical trials often is defined as the return of full syndromal symptomatic criteria. Alternatively, relapse is defined as a HRSD score of $\geq 16$ and an absolute increase of 10 points for at least 1 week (over 2 consecutive visits) (Sackeim et al., 2001).

As mentioned, the response rate to the first antidepressant is approximately $50 \%$ and the remission rate is $37 \%$ (Rush et al., 2006). This rate falls with each successive antidepressant trial, 
reaching $14 \%$ after the third trial and $13 \%$ after the fourth trial according to the Sequenced Treatment Alternatives to Relieve Depression (STAR*D) trial (Rush et al., 2006). A total of 33\% of patients do not achieve symptomatic remission (let alone functional recovery) despite multiple trials of medication (including augmentations with multiple medications)(Rush et al., 2006). These represent millions of Americans each year who do not experience remission of depression, (Kessler et al., 2003; Kessler et al., 1994) and are deemed treatment-resistant. Of the patients that are resistant to conventional therapies, approximately $60 \%$ responded to ECT (Franco-Bronson, 1996; McCall, 2001).

After a successful acute course of ECT, maintaining remission from depressive symptoms is a major challenge for clinicians and patients because the relapse rate has been reported to be as high as $84 \%$ within the first 6 months after ECT (Sackeim et al., 2001). To address this challenge, we performed a systematic review of the literature to best inform the course of continuation treatment.

\section{METHODS}

A systematic literature review of PubMed was performed through April 2014 for clinical trials published in English to determine whether continuation ECT, continuation medication, continuation psychotherapy, or combinations of these are the best strategy to avoid relapse and recurrence of depressive symptoms after an acute course of ECT. Clinical trials comparing $\geq 2$ of the above strategies were included in this systematic review. Keywords used were (ECT OR electroconvulsive therapy OR convulsive therapy) AND (depression OR depressive disorder OR depressive episode) AND (maintenance OR continuation).

\section{RESULTS}

The options for continuation treatment are: (1) pharmacotherapy, (2) psychotherapy, (3) continuation ECT, or (4) a combination of the above interventions.

\section{Continuation pharmacotherapy with or without continuation psychotherapy}

A common practice after an index course ECT is to prescribe antidepressants for continuation therapy (Abou-Saleh \& Coppen, 1988). Although earlier controlled trials of continuation medication alone (C-MED) reported encouraging results, (Kay, Fahy, \& Garside, 1970; Seager \& Bird, 1962) C-MED alone is not effective for all patients, with some studies showing high relapse rates (Prudic et al., 2004; Rasmussen et al., 2007; Sackeim et al., 1990).

Combinations of psychotropics might be better for preventing relapse than a single drug. When a combination of $\geq 2$ drugs is used, lithium augmentation seems to lead to better results than antidepressants alone. This has been shown in a well-designed randomized, double-blinded, placebo-controlled clinical trial that enrolled 290 participants. Of these participants, 159 met 
remission criteria, and 84 (who remitted and agreed to participate) were randomized to 3 arms after remission after acute ECT (Sackeim et al., 2001). Participants were randomized to the continuation arms for 24 weeks after depressive remission to receive nortriptyline alone, a combination of nortriptyline and lithium, or placebo. The study found that a combination of nortriptyline and lithium reduced the relapse rate (39\%) compared with nortriptyline monotherapy (60\%) or with placebo (84\%) over 6 months (Sackeim et al., 2001). Of note in this study, every relapse using nortriptyline-lithium occurred before the 5-week period except for 1 patient.

In another controlled trial after successful index ECT, C-MED resulted in a relapse rate of $51 \%$ within 6 months for treatment as usual, $60 \%$ for protocolized care with nortriptyline, and $39 \%$ for protocolized care with nortriptyline plus lithium (39\%), versus a relapse rate of $84 \%$ for placebo (Tew et al., 2007). This study included 56 randomized patients and was single-blinded.

In clinical practice, personalized treatment decisions are based on clinical, demographic, and logistic characteristics, with consideration of predictors of response, predictors of relapse, patient preferences, and long-term adverse effects. Of note, although cognitive adverse effects can be cause for concern in some cases during an acute course of ECT (especially bilateral ECT), cognitive side effects during C-ECT are not as much of a concern because the sessions are not timed as closely together.

Logistic concerns include the availability of a family or friend who can transport the patient to continuation ECT (C-ECT) sessions and the ability to take time-off (if working) for C-ECT. Factors that predict higher likelihood of relapse after an acute course of ECT are in Table 1. Relapse prevention strategies are in Table 2.

\section{Continuation ECT (C-ECT)}

Because the difference between maintenance ECT and C-ECT is arbitrary, for the purpose of this article we will refer to both as C-ECT. C-ECT indicates any treatment session after the index course administered with an intention to prevent relapse or recurrence of depressive symptoms rather than to induce response/remission of an episode.

Relapse rates with placebo continuation therapy after index ECT are high (Sackeim et al., 2001). Several studies verify the benefits of C-ECT in sustaining remission (Sackeim et al., 1990; Spiker, Stein, \& Rich, 1985). This might be helpful, especially in cases where patients did not respond to several pharmacological agents (Sackeim et al., 1990), are sensitive to medication side effects, or prefer ECT over medication.

A controlled, well-designed trial in this area led by Kellner and colleagues (Consortium for Research in ECT, CORE) evaluated the comparative efficacy of C-ECT vs combination C-MED (lithium carbonate plus nortriptyline) in preventing depressive relapse over a 6-month period after successful bilateral (BL) ECT for acute depression. This randomized, controlled clinical trial enrolled 201 participants in the phase II comparison of C-MED vs C-ECT. The study was single- 
blinded for neuropsychological tests. This study found equal sustained remission rate of $46 \%$ throughout the study period for both the C-MED and the C-ECT arm of the study (Kellner et al., 2006).

In a recent meta-analysis and systematic review of literature on post-ECT relapse that included 32 studies, the authors reported that in modern studies of C-MED, 51\% of patients relapsed by 12 months after a successful acute course of ECT. A total of $37.7 \%$ of patients who relapsed did so within the first 6 months. The relapse rate at 6 months with C-ECT was similar to C-MED (37.2\%) (Jelovac, Kolshus, \& McLoughlin, 2013).

Another recent literature review on C-ECT concluded that ECT is "an underused treatment option that can substantially reduce risks of relapse"(Rabheru, 2012). The factors favoring consideration of C-ECT (with or without pharmacotherapy) are listed in Table 3.

\section{Combination of C-ECT and pharmacotherapy or psychotherapy}

A general principle with antidepressant therapy is that treatment for the acute phase also is effective as continuation treatment. However, after acute response to ECT, the most common clinical practice is to stop ECT and to use only medications as continuation therapy (Aronson, Shukla, \& Hoff, 1987) despite the fact that some patients who undergo ECT have not responded to medications or psychotherapy before ECT.

Long-term remission may be preserved with combined C-ECT and pharmacotherapy, especially when symptoms did not respond to medication before ECT. Retrospective studies of hospitalization rates have reported lower rates of psychiatric hospitalization in patients receiving C-ECT with medications compared with admission rates before receiving ECT alone (Russell et al., 2003; Thornton, Mulsant, Dealy, \& Reynolds, 1990).

Another retrospective study comparing C-ECT plus medication (C-ECT group) versus CMED alone (C-MED group) demonstrated a significant advantage for the C-ECT group (Gagne, Furman, Carpenter, \& Price, 2000). The 2-year cumulative probability of surviving without relapse

or recurrence for the ECT group was $93 \%$ while the 2-year cumulative probability of surviving without relapse or recurrence was only $52 \%$ for the C-MED group. At 5 years, the C-ECT group had relapse-/recurrence-free survival of $73 \%$, while the C-MED group had relapse-/recurrencefree survival of $18 \%$. The weaknesses of this study included its retrospective nature. Although on the high end for ECT studies, sample size was modest $(\mathrm{N}=59)$ to allow for adjustments for some of the potential confounding variables. Because of the retrospective nature, there was no random assignment of the groups or blinding. The mean survival time for staying well for the CECT group was 6.9 years, while for the C-MED group it was 2.7 years (Gagne et al., 2000). These results support the superior efficacy of C-ECT plus medications compared with C-MED alone in preventing relapse and recurrence in patients with depression who have responded to acute ECT. 
A randomized trial by Brakemeier et al. (Brakemeier et al., 2013) examined the most efficacious continuation intervention for patients who responded to acute ECT. After responsenot remission - to acute right unilateral (RUL) ultra-brief ECT, 60 patients were randomized to cognitive-behavioral therapy (C-CBT) plus "guideline-based" C-MED, C-ECT (RUL ultra-brief) plus C-MED, or C-MED alone. The study found superiority in the C-CBT group on the proportion of patients who "remained well" after 12 months (primary outcome). In this study, $70 \%$ of the initial 90 patients responded to acute ECT, and $47 \%$ remitted. After 6 months, sustained response rates were $77 \%$ in the C-CBT arm, 40\% in the C-ECT arm, and 44\% in the C-MED alone arm. After 12 months, sustained response rates were $65 \%$ in the C-CBT group, $28 \%$ in the C-ECT group, and 33\% in the C-MED alone group. The authors concluded that "ultra-brief pulse ECT as a continuation treatment correlates with low sustained response rates," and that C-CBT plus C-MED "might be an effective continuation treatment to sustain response after successful ECT in MDD patients".

During the initial acute ECT phase, participants were switched to BLECT if they did not respond to RUL ECT. Nonetheless, during the randomization continuation phase, all participants in the C-ECT arm were given RUL ECT. It is not clear how many of the participants who had BLECT were treated later with RUL ECT during the continuation phase, but this could make ECT appear to be ineffective during the continuation phase, because the BL ECT that was previously effective was discontinued. For the above reasons, it is hard to draw conclusions on the superior efficacy of C-CBT compared with the ultra-brief unilateral ECT used in this study without more rigorous replication of this study.

Another small prospective study examined 21 participants who received C-ECT (12 depressed and 8 schizoaffective) and 21 matched controls (received C-MED) who refused C-ECT. This study compared patients who received C-ECT plus pharmacotherapy with those who received C-MED alone. Limitations of this study include small sample size, no random assignment, no blinding, and the C-ECT group was not homogenous including both RUL and BL ECT and mixed diagnoses. However, the study was prospective and had a matched control group. The study found a significantly better outcome for patients receiving C-ECT plus pharmacotherapy compared with C-MED alone in terms of time to rehospitalization (Swoboda, Conca, Konig, Waanders, \& Hansen, 2001).

Another prospective, randomized, single-blind trial assessed tolerability and efficacy of continuation treatments for geriatric patients with psychotic depression after acute ECT remission (Navarro et al., 2008). The researchers compared the 2-year period outcome of 2 groups of geriatric patients with psychotic major depression who remitted after acute treatment of ECT plus nortriptyline. During the continuation phase, one group was randomized to nortriptyline alone (C-MED) $(\mathrm{N}=17)$, and the other group was randomized to C-ECT plus nortriptyline $(\mathrm{N}=16)(\mathrm{C}-\mathrm{ECT})$. Over the 2 years of treatment, the mean survival time of remission was significantly longer for the combined C-ECT plus C-MED group compared with the C-MED group. There was no difference in tolerability between the 2 groups. The researchers concluded 
that the study supported the use of combined C-ECT plus antidepressant treatment in geriatric patients with psychotic major depression who remitted using acute ECT.

Another prospective, multicenter, randomized, controlled trial tested the "hypothesis that relapse prevention with continuation ECT plus pharmacotherapy is more effective than pharmacotherapy alone" after a course of acute ECT for major depressive episode (unipolar or bipolar depression)(Nordenskjold et al., 2013). The study randomized 2 parallel groups (after response to acute ECT) in 4 hospitals in Sweden. Participants $(N=56)$ were randomly assigned to either 29 C-ECT sessions with C-MED or C-MED alone for 1 year. The C-MED consisted of antidepressants (98\%), lithium (56\%), and antipsychotics (30\%). The primary outcome was relapse within 1 year. (Relapse was defined as $\geq 20$ points on the Montgomery-Åsberg Depression Rating Scale or inpatient psychiatric care or suicide or suspected suicide.) Sixty-one percent of participants relapsed within 1 year in the C-MED alone arm versus $32 \%$ of those in the C-ECT plus C-MED arm $(P=.036)$. (The Cox proportional hazard ratio was 2.32 (95\% confidence interval, 1.03-5.22).) In the C-MED arm, there was 1 suspected suicide by intoxication (died from intoxication, the suicidal intent was not proven but was considered in the study as a suspected suicide) and 3 suicide attempts but none in the combined C-ECT arm. The authors concluded that there was a "statistically significant advantage for combined treatment with pharmacotherapy and continuation ECT".

The Prolonged Remission in Depressed Elderly (PRIDE) study is an ongoing multicenter, randomized clinical trial by the CORE group mentioned above. The PRIDE study examines whether combined pharmacotherapy (lithium plus venlafaxine) plus C-ECT, or combined lithium plus venlafaxine C-MED alone, is more effective in maintaining remission in depressed older patients with MDD after acute ECT. Data from this study will be valuable in informing clinical design-making of combining ECT with these medications.

In summary, most studies have shown that combined C-ECT plus continuation pharmacotherapy (C-MED) have significantly better results in relapse prevention than either alone (Navarro et al., 2008; Nordenskjold et al., 2013; Swoboda et al., 2001). One study showed that C-CBT combined with C-MED is better than C-ECT combined with C-MED. However, it is hard to draw conclusions or recommend C-CBT as superior to ECT based on a single study because of design limitation, and results that are different from most of the literature.

\section{Other considerations in continuation ECT and pharmacotherapy in clinical practice}

There are other important points that need to be considered in the routine clinical aspects of continuation/maintenance therapy. First, it is helpful to have a rating scale to consistently and systematically assess improvement or relapse in depressive symptoms during acute ECT and continuation treatment. Although many clinical trials have used HRSD, clinicians can use simpler, self-administered scales, including the 9-item Patient Health Questionnaire (Spitzer, Kroenke, \& Williams, 1999). 
Second, clinicians should individualize treatment decisions by weighing risks and benefits of psychotropics, especially lithium, among other alternatives for treatment-resistant depressive episodes of unipolar and bipolar disorders. Lithium can have several side effects including nausea, thyroid dysfunction, and renal dysfunction, and blood level monitoring (for lithium level, renal, and thyroid functions) is required. However, if used with caution and blood tests are checked regularly, most patients tolerate the drug well (Mauri et al., 1999). The risk from chronic lithium use includes renal toxicity (McCann, Daly, \& Kelly, 2008). If kidney function is monitored closely, however, lithium generally can be used safely in most cases, even for long periods of time (McCann et al., 2008; Tredget, Kirov, \& Kirov, 2010). Also, a systematic review and meta-analysis of 23 studies concluded that "any lithium-associated increase in serum creatinine is quantitatively small and of questionable clinical significance" (Paul, Minay, Cardwell, Fogarty, \& Kelly, 2010). However, routine monitoring of renal function is essential. Lithium augmentation plays an important role for managing treatment-resistant depression(Bauer et al., 2010). It has potential benefits not only in treatment-resistant depression (Licht, 2012), but also in reducing suicide (Schou, 1999) in bipolar and unipolar depression (Cipriani, Hawton, Stockton, \& Geddes, 2013; Muller-Oerlinghausen, Berghofer, \& Ahrens, 2003). The drug also has a neuroprotective effect (Chiu \& Chuang, 2011).

Third, although cognitive side effects - which can be of concern during acute ECT-are a possibility, they usually improve with time. Acute side effects of ECT have been discussed extensively elsewhere (American Psychiatric Association (2000)) and are not the focus of this article. For the purposes of this article, C-ECT has less cognitive side effects than acute ECT, simply because of the timing of treatment sessions. Among C-ECT patients, there likely is additional cognitive sparing as the treatment sessions are moved farther and farther apart. However, although this represents our clinical experience and the general consensus in the field, it has not been verified by empirical research. There are no studies comparing cognitive effects of ECT with session spacing during C-ECT, for example between patients getting ECT once per week versus once per month.

Fourth, one of the barriers to pharmacotherapy is the high rates of medication nonadherence (Lopez-Torres, Parraga, Del Campo, \& Villena, 2013). Adherence to antidepressant treatment may range from $30 \%$ to $70 \%$ (Barbui et al., 2000). A study by Cramer and Rosenheck (Cramer \& Rosenheck, 1998) examining pooled data reports that adherence rates for antidepressants rarely exceed $65 \%$. Others found $37 \%$ of patients discontinued antidepressants by 12 weeks in the counselled group compared with 61\% in the non-counselled group (Peveler, George, Kinmonth, Campbell, \& Thompson, 1999). Another study found that only $20 \%$ to $34 \%$ of individuals filled $\geq 4$ prescriptions (Katon, von Korff, Lin, Bush, \& Ormel, 1992).

Physicians cannot predict medication adherence in many patients (Lopez-Torres et al., 2013). Non-adherence is commonly encountered in outpatient care not only with older medications such as tricyclic antidepressants, but also with newer medications such as selective serotonin reuptake inhibitors (Barbui et al., 2000). However, studies indicate that adherence for 
C-MED might be lesser than outpatient C-ECT. Dew and McCall found an outpatient C-ECT adherence rate of $91 \%$ (Dew \& McCall, 2004). This is could be attributed to ECT being a more potent and rapidly acting form of treatment for treatment-resistant depressive episodes and/or suicidality.

As directions for further research, we recommend that further investigations in refinements of C-ECT and continuation pharmacotherapeutic combinations-and psychotherapy especially in patients who were non-treatment resistant to psychotherapy-with ECT are needed to further improve relapse prevention strategies after index ECT, especially for treatmentresistant mood disorders.

\section{CONCLUSIONS}

Although there are few rigorous randomized clinical trials in this area, the current literature suggests that combined C-ECT and pharmacotherapy may be more effective than either alone in preventing relapse. However, more definitive randomized clinical trials to guide the best strategies to avoid relapse after an acute course of index ECT are needed. 
Disclosures: Dr. Youssef received support from the National Institute of Mental Health Grant No. $1 \mathrm{U} 01 \mathrm{MH084241}$ for the Prolonged Remission in Depressed Elderly (PRIDE) ECT clinical trial. Dr. McCall received research support from the National Institute of Mental Health Grant No. 1U01MH086127-01 for the Prolonged Remission in Depressed Elderly (PRIDE) ECT clinical trial; and the National Institute of Mental Health Grant No. 1 R01 MH095776-01A1; and from Merck Sharp \& Dohme Corp. Dr. McCall received royalties from Wolters Kluwer Publishing. 


\section{Table 1}

\section{Common relapse predictors}

1. Medication resistance

2. Female sex

3. Residual depressive symptoms

4. Comorbid untreated psychiatric disorders such as posttraumatic stress disorder, substance use disorder, or personality disorder

5. History of unsuccessful treatment with ECT if the treatment parameter and electrode placement were optimized

6. Highly recurrent major depressive disorder

7. Depressive symptoms as part of a rapid cycling bipolar disorder

8. Uncontrolled medical condition that is inducing or exacerbating the mood disorder such as untreated hypothyroidism or chronic severe pain disorder 


\section{Table 2}

\section{Relapse prevention strategies}

1. Starting continuation pharmacotherapy during the index course

2. Aggressively treating residual symptoms after an index course of ECT

3. Tailoring C-ECT session to the level of symptoms and clinical presentation without over or under treatment, rather than using a fixed schedule for continuation ECT

4. Combined ECT and pharmacotherapy including possibility of lithium augmentation

5. Gradually discontinuing (or minimizing if absolutely necessary) benzodiazepine use and other CNS depressants to both avoid the depressogenic effect of these substances as well as to avoid interference with seizure induction during ECT

6. Treating other comorbid psychiatric conditions (formerly Axis I disorders) that might be hindering treatment such as posttraumatic stress disorder, substance use comorbidity etc.

7. Treating comorbid personality disorder that might be hindering treatment such as treating borderline personality disorder with dialectical behavioral therapy

8. Treating comorbid medical condition that could be exacerbating (or inducing) mood disorder such as treating hypothyroidism

C-ECT: continuation electroconvulsive therapy; ECT: electroconvulsive therapy. 


\section{Table 3}

\section{Factors favoring consideration of continuation ECT}

1. Positive response to index ECT

2. Patient preference

3. Failure of pharmacotherapy and psychotherapy to achieve remission in the past

4. Intolerance to effective pharmacological agents or side effects unacceptable to the patient

5. Limited medical illness that could increase risk of further ECT or anesthesia

6. Trials to discontinue ECT result in rapid reemergence of depressive symptoms despite optimization of pharmacological interventions and/or pharmacotherapy

7. Potential for high stakes complications is there were a relapse, i.e., return of intense suicidality or catatonia

8. No barriers to the logistic issues in complying with outpatient ECT (such as transportation and availability of a friend or family to be involved in care; ability to take a day off for the ECT procedure, if applicable)

ECT: electroconvulsive therapy. 


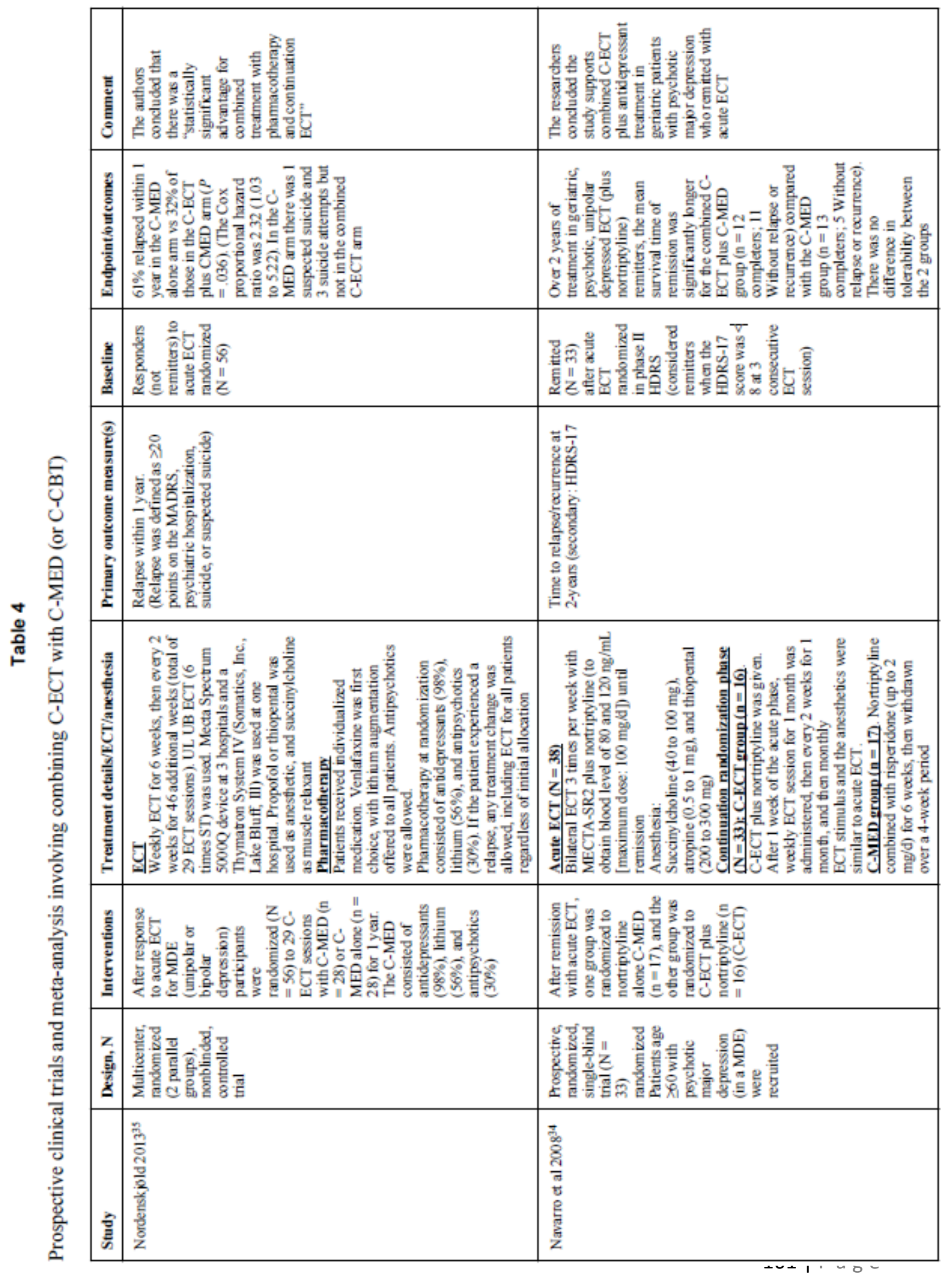




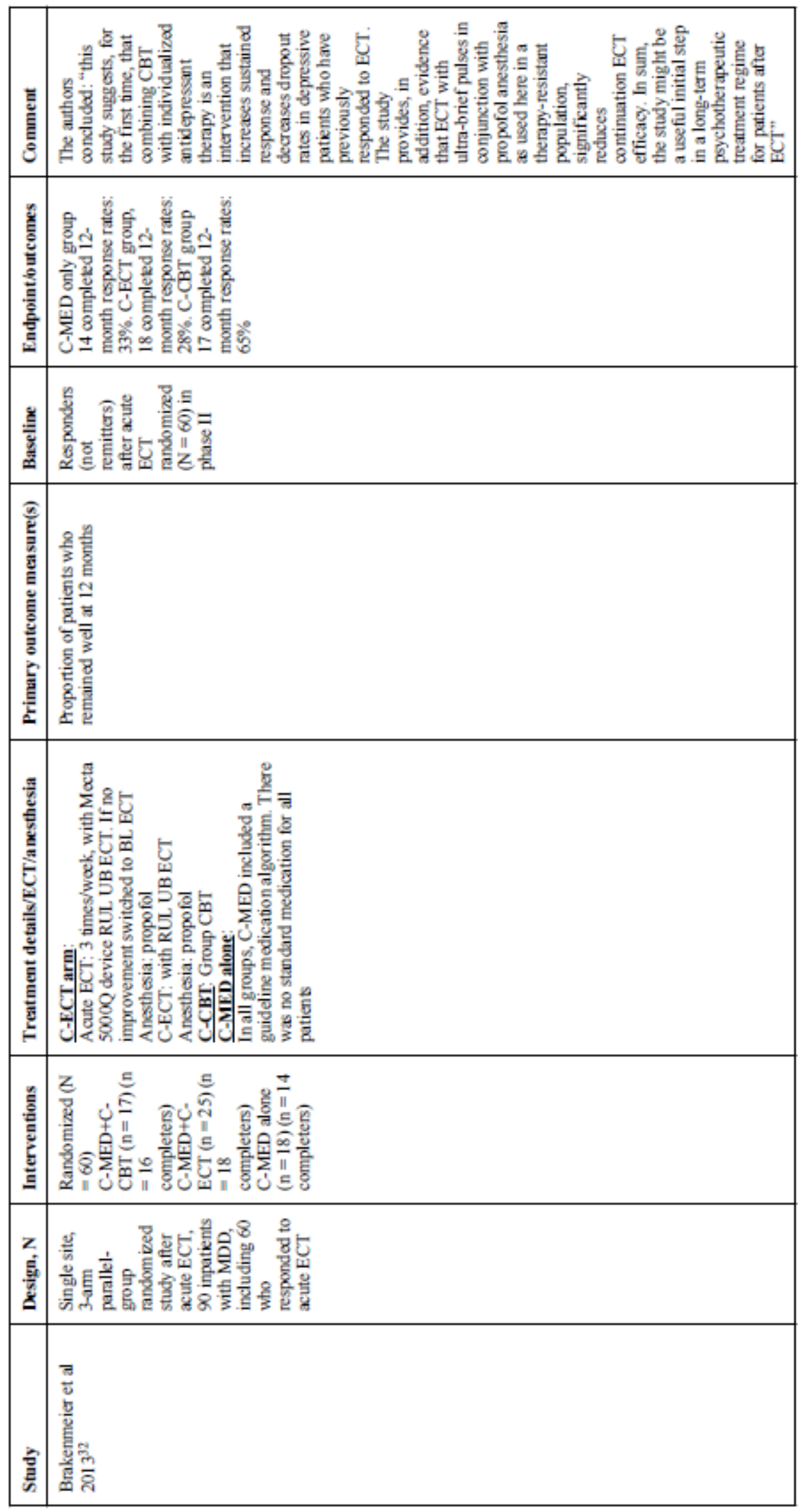

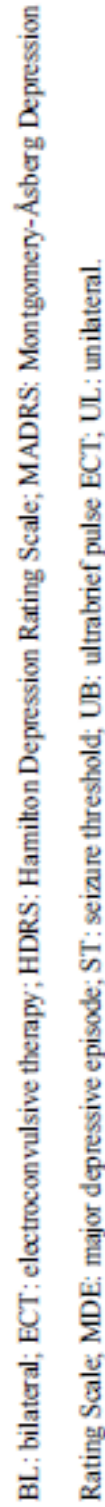

162 | P a g e 
Contributions (added for the purpose of this thesis): Nagy Youssef conceptualized the idea of this systematic review and developed the inclusion criteria and search strategy, searched the literature, and wrote the initial draft of the manuscript. Both Nagy Youssef and Vaughn McCall contributed intellectually and edited the later versions of the paper. Both coauthors approved the final version of the manuscript. 


\section{REFERENCES}

Abou-Saleh, M. T., \& Coppen, A. J. (1988). Continuation therapy with antidepressants after electroconvulsive therapy. Convuls Ther, 4(4), 263-268. Retrieved from http://www.ncbi.nlm.nih.gov/pubmed/11940975

American Psychiatric Association. (2000). Practice guideline for the treatment of patients with major depressive disorder (revision). Am J Psychiatry, 157(4 Suppl), 1-45. Retrieved from http://www.ncbi.nlm.nih.gov/pubmed/10767867

Aronson, T. A., Shukla, S., \& Hoff, A. (1987). Continuation therapy after ECT for delusional depression: a naturalistic study of prophylactic treatments and relapse. Convuls Ther, 3(4), 251-259. Retrieved from http://www.ncbi.nlm.nih.gov/pubmed/11940926

Barbui, C., Hotopf, M., Freemantle, N., Boynton, J., Churchill, R., Eccles, M. P., . . Mason, J. M. (2000). Selective serotonin reuptake inhibitors versus tricyclic and heterocyclic antidepressants: comparison of drug adherence. Cochrane Database Syst Rev(4), CD002791. doi:10.1002/14651858.CD002791

Bauer, M., Adli, M., Bschor, T., Pilhatsch, M., Pfennig, A., Sasse, J., . . Lewitzka, U. (2010). Lithium's emerging role in the treatment of refractory major depressive episodes: augmentation of antidepressants. Neuropsychobiology, 62(1), 36-42. doi:10.1159/000314308

Brakemeier, E. L., Merkl, A., Wilbertz, G., Quante, A., Regen, F., Buhrsch, N., . . Bajbouj, M. (2013). Cognitive-behavioral therapy as continuation treatment to sustain response after electroconvulsive therapy in depression: a randomized controlled trial. Biol Psychiatry, 76(3), 194-202. doi:10.1016/j.biopsych.2013.11.030

Chiu, C. T., \& Chuang, D. M. (2011). Neuroprotective action of lithium in disorders of the central nervous system. Zhong Nan Da Xue Xue Bao Yi Xue Ban, 36(6), 461-476. doi:10.3969/j.issn.1672-7347.2011.06.001

Cipriani, A., Hawton, K., Stockton, S., \& Geddes, J. R. (2013). Lithium in the prevention of suicide in mood disorders: updated systematic review and meta-analysis. BMJ, 346, f3646. doi:10.1136/bmj.f3646

Coppen, A., Abou-Saleh, M. T., Milln, P., Bailey, J., Metcalfe, M., Burns, B. H., \& Armond, A. (1981). Lithium continuation therapy following electroconvulsive therapy. $\mathrm{Br} \mathrm{J}$ Psychiatry, 139(4), 284-287. doi:10.1192/bjp.139.4.284

Cramer, J. A., \& Rosenheck, R. (1998). Compliance with medication regimens for mental and physical disorders. Psychiatr Serv, 49(2), 196-201. doi:10.1176/ps.49.2.196

Davidson, J. R. (2010). Major depressive disorder treatment guidelines in America and Europe. J Clin Psychiatry, 71 (Suppl E1), e04. doi:10.4088/JCP.9058se1c.04gry

Dew, R., \& McCall, W. V. (2004). Efficiency of outpatient ECT. J ect, 20(1), 24-25. Retrieved from http://www.ncbi.nlm.nih.gov/pubmed/15087993 
Driessen, E., Van, H. L., Don, F. J., Peen, J., Kool, S., Westra, D., . . Dekker, J. J. (2013). The efficacy of cognitive-behavioral therapy and psychodynamic therapy in the outpatient treatment of major depression: a randomized clinical trial. Am J Psychiatry, 170(9), 1041-1050. doi:10.1176/appi.ajp.2013.12070899

Dukakis, K., \& Tye, L. (2007). Shock. New York: Penguin.

Franco-Bronson, K. (1996). The management of treatment-resistant depression in the medically ill. Psychiatr Clin North Am, 19(2), 329-350. doi:10.1016/S0193-953X(05)70291-4

Frank, E., Prien, R. F., Jarrett, R. B., Keller, M. B., Kupfer, D. J., Lavori, P. W., . . Weissman, M. M. (1991). Conceptualization and rationale for consensus definitions of terms in major depressive disorder. Remission, recovery, relapse, and recurrence. Arch Gen Psychiatry, 48(9), 851-855. doi:10.1001/archpsyc.1991.01810330075011

Gagne, G. G., Jr., Furman, M. J., Carpenter, L. L., \& Price, L. H. (2000). Efficacy of continuation ECT and antidepressant drugs compared to long-term antidepressants alone in depressed patients. Am J Psychiatry, 157(12), 1960-1965. doi:10.1176/appi.ajp.157.12.1960

Jelovac, A., Kolshus, E., \& McLoughlin, D. M. (2013). Relapse following successful electroconvulsive therapy for major depression: a meta-analysis. Neuropsychopharmacology, 38(12), 2467-2474. doi:10.1038/npp.2013.149

Katon, W., von Korff, M., Lin, E., Bush, T., \& Ormel, J. (1992). Adequacy and duration of antidepressant treatment in primary care. Med Care, 30(1), 67-76. Retrieved from http://www.ncbi.nlm.nih.gov/pubmed/1729588

Kay, D. W., Fahy, T., \& Garside, R. F. (1970). A seven-month double-blind trial of amitriptyline and diazepam in ECT-treated depressed patients. Br J Psychiatry, 117(541), 667-671. doi:10.1192/bjp.117.541.667

Kellner, C. H., Knapp, R. G., Petrides, G., Rummans, T. A., Husain, M. M., Rasmussen, K., . . Fink, M. (2006). Continuation electroconvulsive therapy vs pharmacotherapy for relapse prevention in major depression: a multisite study from the Consortium for Research in Electroconvulsive Therapy (CORE). Arch Gen Psychiatry, 63(12), 1337-1344. doi:10.1001/archpsyc.63.12.1337

Kessler, R. C., Berglund, P., Demler, O., Jin, R., Koretz, D., Merikangas, K. R., . . Wang, P. S. (2003). The epidemiology of major depressive disorder: results from the National Comorbidity Survey Replication (NCS-R). Jama, 289(23), 3095-3105. doi:10.1001/jama.289.23.3095

Kessler, R. C., Chiu, W. T., Demler, O., Merikangas, K. R., \& Walters, E. E. (2005). Prevalence, severity, and comorbidity of 12-month DSM-IV disorders in the National Comorbidity Survey Replication. Arch Gen Psychiatry, 62(6), 617-627. doi:10.1001/archpsyc.62.6.617

Kessler, R. C., McGonagle, K. A., Zhao, S., Nelson, C. B., Hughes, M., Eshleman, S., . . Kendler, K. S. (1994). Lifetime and 12-month prevalence of DSM-III-R psychiatric disorders in the 
United States. Results from the National Comorbidity Survey. Arch Gen Psychiatry, 51(1), 8-19. doi:10.1001/archpsyc.1994.03950010008002

Licht, R. W. (2012). Lithium: still a major option in the management of bipolar disorder. CNS Neurosci Ther, 18(3), 219-226. doi:10.1111/j.1755-5949.2011.00260.x

Lopez-Torres, J., Parraga, I., Del Campo, J. M., \& Villena, A. (2013). Follow up of patients who start treatment with antidepressants: treatment satisfaction, treatment compliance, efficacy and safety. BMC Psychiatry, 13, 65. doi:10.1186/1471-244X-13-65

Mauri, M. C., Laini, V., Scalvini, M. E., Volonteri, L. S., Ferrari, M. S., \& Panza, G. (1999). Lithium safety in the prophylaxis of bipolar disorders: a study with plasma levels. Eur Rev Med Pharmacol Sci, 3(2), 63-69. Retrieved from http://www.ncbi.nlm.nih.gov/pubmed/10827806

McCall, W. V. (2001). Electroconvulsive therapy in the era of modern psychopharmacology. Int J Neuropsychopharmacol, 4(3), 315-324. doi:10.1017/S1461145701002437

McCann, S. M., Daly, J., \& Kelly, C. B. (2008). The impact of long-term lithium treatment on renal function in an outpatient population. Ulster Med J, 77(2), 102-105. Retrieved from http://www.ncbi.nlm.nih.gov/pubmed/18711626

Muller-Oerlinghausen, B., Berghofer, A., \& Ahrens, B. (2003). The antisuicidal and mortalityreducing effect of lithium prophylaxis: consequences for guidelines in clinical psychiatry. Can J Psychiatry, 48(7), 433-439. doi:10.1177/070674370304800702

Navarro, V., Gasto, C., Torres, X., Masana, G., Penades, R., Guarch, J., . . Catalan, R. (2008). Continuation/maintenance treatment with nortriptyline versus combined nortriptyline and ECT in late-life psychotic depression: a two-year randomized study. Am J Geriatr Psychiatry, 16(6), 498-505. doi:10.1097/JGP.0b013e318170a6fa

Nordenskjold, A., von Knorring, L., Ljung, T., Carlborg, A., Brus, O., \& Engstrom, I. (2013). Continuation electroconvulsive therapy with pharmacotherapy versus pharmacotherapy alone for prevention of relapse of depression: a randomized controlled trial. J ect, 29(2), 86-92. doi:10.1097/YCT.0b013e318276591f

Paul, R., Minay, J., Cardwell, C., Fogarty, D., \& Kelly, C. (2010). Meta-analysis of the effects of lithium usage on serum creatinine levels. J Psychopharmacol, 24(10), 1425-1431. doi:10.1177/0269881109104930

Petrides, G., Fink, M., Husain, M. M., Knapp, R. G., Rush, A. J., Mueller, M., . . Kellner, C. H. (2001). ECT remission rates in psychotic versus nonpsychotic depressed patients: a report from CORE. J ect, 17(4), 244-253. Retrieved from http://www.ncbi.nlm.nih.gov/pubmed/11731725

Peveler, R., George, C., Kinmonth, A. L., Campbell, M., \& Thompson, C. (1999). Effect of antidepressant drug counselling and information leaflets on adherence to drug treatment in primary care: randomised controlled trial. BMJ, 319(7210), 612-615. doi:10.1136/bmj.319.7210.612 
Prudic, J., Olfson, M., Marcus, S. C., Fuller, R. B., \& Sackeim, H. A. (2004). Effectiveness of electroconvulsive therapy in community settings. Biol Psychiatry, 55(3), 301-312. doi:10.1016/j.biopsych.2003.09.015

Rabheru, K. (2012). Maintenance electroconvulsive therapy (M-ECT) after acute response: examining the evidence for who, what, when, and how? J ect, 28(1), 39-47. doi:10.1097/YCT.0b013e3182455758

Rasmussen, K. G., Knapp, R. G., Biggs, M. M., Smith, G. E., Rummans, T. A., Petrides, G., . . Kellner, C. H. (2007). Data management and design issues in an unmasked randomized trial of electroconvulsive therapy for relapse prevention of severe depression: the Consortium for Research in Electroconvulsive Therapy Trial. J ect, 23(4), 244-250. doi:10.1097/yct.0b013e31814515d6

Rush, A. J., Trivedi, M. H., Wisniewski, S. R., Nierenberg, A. A., Stewart, J. W., Warden, D., . . Fava, M. (2006). Acute and longer-term outcomes in depressed outpatients requiring one or several treatment steps: a STAR*D report. Am J Psychiatry, 163(11), 1905-1917. doi:10.1176/appi.ajp.163.11.1905

Russell, J. C., Rasmussen, K. G., O'Connor, M. K., Copeman, C. A., Ryan, D. A., \& Rummans, T. A. (2003). Long-term maintenance ECT: a retrospective review of efficacy and cognitive outcome. J ect, 19(1), 4-9. Retrieved from http://www.ncbi.nlm.nih.gov/pubmed/12621270

Sackeim, H. A., Haskett, R. F., Mulsant, B. H., Thase, M. E., Mann, J. J., Pettinati, H. M., . . . Prudic, J. (2001). Continuation pharmacotherapy in the prevention of relapse following electroconvulsive therapy: a randomized controlled trial. Jama, 285(10), 1299-1307. doi:10.1001/jama.285.10.1299

Sackeim, H. A., Prudic, J., Devanand, D. P., Decina, P., Kerr, B., \& Malitz, S. (1990). The impact of medication resistance and continuation pharmacotherapy on relapse following response to electroconvulsive therapy in major depression. J Clin Psychopharmacol, 10(2), 96-104. Retrieved from http://www.ncbi.nlm.nih.gov/pubmed/2341598

Sackeim, H. A., Prudic, J., Devanand, D. P., Nobler, M. S., Lisanby, S. H., Peyser, S., . . Clark, J. (2000). A prospective, randomized, double-blind comparison of bilateral and right unilateral electroconvulsive therapy at different stimulus intensities. Arch Gen Psychiatry, 57(5), 425-434. doi:10.1001/archpsyc.57.5.425

Schou, M. (1999). Perspectives on lithium treatment of bipolar disorder: action, efficacy, effect on suicidal behavior. Bipolar Disord, 1(1), 5-10. doi:10.1034/j.1399-5618.1999.10103.x

Seager, C. P., \& Bird, R. L. (1962). Imipramine with electrical treatment in depression--a controlled trial. J Ment Sci, 108, 704-707. doi:10.1192/bjp.108.456.704

Spiker, D. G., Stein, J., \& Rich, C. L. (1985). Delusional depression and electroconvulsive therapy: one year later. Convuls Ther, 1(3), 167-172. Retrieved from http://www.ncbi.nlm.nih.gov/pubmed/11940820 
Spitzer, R. L., Kroenke, K., \& Williams, J. B. (1999). Validation and utility of a self-report version of PRIME-MD: the PHQ primary care study. Primary Care Evaluation of Mental Disorders. Patient Health Questionnaire. Jama, 282(18), 1737-1744. doi:10.1001/jama.282.18.1737

Swoboda, E., Conca, A., Konig, P., Waanders, R., \& Hansen, M. (2001). Maintenance electroconvulsive therapy in affective and schizoaffective disorder. Neuropsychobiology, 43(1), 23-28. doi:10.1159/000054861

Tew, J. D., Jr., Mulsant, B. H., Haskett, R. F., Joan, P., Begley, A. E., \& Sackeim, H. A. (2007). Relapse during continuation pharmacotherapy after acute response to ECT: a comparison of usual care versus protocolized treatment. Ann Clin Psychiatry, 19(1), 1-4. doi:10.1080/10401230601163360

Thornton, J. E., Mulsant, B. H., Dealy, R., \& Reynolds, C. F., 3rd. (1990). A retrospective study of maintenance electroconvulsive therapy in a university-based psychiatric practice. Convuls Ther, 6(2), 121-129. Retrieved from http://www.ncbi.nlm.nih.gov/pubmed/11941053

Tredget, J., Kirov, A., \& Kirov, G. (2010). Effects of chronic lithium treatment on renal function. J Affect Disord, 126(3), 436-440. doi:10.1016/j.jad.2010.04.018

World Health Organization. (2008). Table A2: Burden of disease in DALYs by cause, sex and income group in WHO regions, estimates for 2004. In The global burden of disease: 2004 update. Geneva: WHO Press; Retrieved from https://www.who.int/healthinfo/global burden disease/GBD report 2004update full. pdf. 


\section{Chapter 9 Effects of Continuation Electroconvulsive Therapy on Quality of Life in Elderly Depressed Patients: A Randomized Clinical Trial}

W. Vaughn McCall, M.D., M.S., ${ }^{a}$ Sarah H. Lisanby, M.D., ${ }^{\text {b }}$ Peter B. Rosenquist, M.D., ${ }^{a}$ Mary Dooley, M.S., ${ }^{c}$ Mustafa M. Husain, M.D., ${ }^{d}$ Rebecca G. Knapp, Ph.D., ${ }^{c}$ Georgios Petrides, M.D., Matthew V. Rudorfer, M.D., ${ }^{b}$ Robert C. Young, M.D., ${ }^{f}$ Shawn M. McClintock, Ph.D., M.S.C.S., ${ }^{d}$

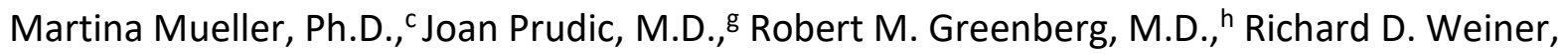

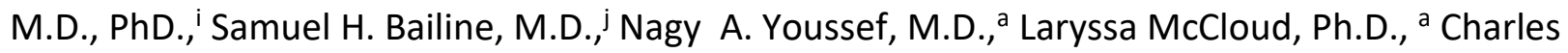
H. Kellner, M.D., ${ }^{k}$ and the CORE/PRIDE Work Group.

a) Department of Psychiatry and Health Behavior; Medical College of Georgia; Augusta University, Augusta, Georgia

b) National Institute of Mental Health, Bethesda, Maryland

c) Medical University of South Carolina, Charleston, South Carolina

d) University of Texas Southwestern Medical Center, Dallas, Texas

e) Hofstra Northwell School of Medicine, Hempstead, New York

f) New York Presbyterian/Weill Cornell Medical Center, New York, New York

g) Department of Psychiatry, Columbia University, New York, New York

h) New York University Langone Medical Center, New York, New York

i) Department of Psychiatry, Duke University School of Medicine, Durham, North Carolina

j) Zucker Hillside Hospital, Glen Oaks, New York

k) Icahn School of Medicine at Mount Sinai, New York, New York

Address correspondence to Dr. W Vaughn McCall; Department of Psychiatry and Health Behavior; Medical College of Georgia; Augusta University; 997 St. Sebastian Way; Augusta, Georgia, 30912. Phone 706-721-6719. Fax 706-721-1793 (wmccall@augusta.edu).

Published as: McCall WV, Lisanby SH, Rosenquist PB, Dooley M, Husain MM, Knapp RG, Petrides G, Rudorfer MV, Young RC, McClintock SM, Mueller M, Prudic J, Greenberg RM, Weiner RD, Bailine SH, Youssef NA, McCloud L, Kellner CH; CORE/PRIDE Work Group. Effects of continuation electroconvulsive therapy on quality of life in elderly depressed patients: A randomized clinical trial. J Psychiatr Res. 2018 Feb;97:65-69. doi: 10.1016/j.jpsychires.2017.11.001. Epub 2017 Nov

16. PubMed PMID: 29195125; PubMed Central PMCID: PMC5742556. https://www.sciencedirect.com/science/article/pii/S0022395617309846?via\%3Dihub 


\section{$\underline{\text { Abstract }}$}

We examined whether electroconvulsive therapy (ECT) plus medications ("STABLE + PHARM" group) had superior health-related quality of life (HRQOL) compared with medications alone ("PHARM" group) as continuation strategy after successful acute right unilateral ECT for major depressive disorder (MDD). We hypothesized that scores from the Medical Outcomes Study Short Form-36 (SF-36) would be higher during continuation treatment in the "STABLE + PHARM" group versus the "PHARM" group. The overall study design was called "Prolonging Remission in Depressed Elderly" (PRIDE). Remitters to the acute course of ECT were re-consented to enter a 6-month RCT of "STABLE + PHARM" versus "PHARM". Measures of depressive symptoms and cognitive function were completed by blind raters; SF-36 measurements were patient self-report every 4 weeks.

Participants were 120 patients $\geq 60$ years old. Patients with dementia, schizophrenia, bipolar disorder, or substance abuse were excluded. The "PHARM" group received venlafaxine and lithium. The "STABLE + PHARM" received the same medications, plus 4 weekly outpatient ECT sessions, with additional ECT session as needed. Participants were mostly female (61.7\%) with a mean age of $70.5 \pm 7.2$ years. "STABLE + PHARM" patients received $4.5 \pm 2.5 \mathrm{ECT}$ sessions during Phase 2. "STABLE + PHARM" group had 7 point advantage $(3.5-10.4,95 \% \mathrm{Cl})$ for Physical Component Score of SF-36 ( $\mathrm{P}<0.0001)$, and 8.2 point advantage $(4.2-12.2,95 \% \mathrm{Cl})$ for Mental Component Score ( $P$ <0.0001). Additional ECT resulted in overall net health benefit. Consideration should be given to administration of additional ECT to prevent relapse during the continuation phase of treatment of MDD.

\section{Keywords}

Electroconvulsive therapy; quality of life; randomized controlled trial; major depressive disorder; continuation therapy

TRIAL REGISTRATION: Clinical Trials.gov NCT01028508 


\section{INTRODUCTION}

Major Depressive Disorder (MDD) is a leading cause of poor health-related quality of life (HRQOL)(World Health Organization, 2011). The HRQOL deficits increase with depression symptom severity (W. Vaughn McCall, Wayne Cohen, Beth Reboussin, \& Powell Lawton, 1999). Age influences the HRQOL deficit patterns, with younger depressed patients reporting more problems with relationships and older depressed patients reporting more problems with daily living and role functioning (W. Vaughn McCall et al., 1999). A third of depressed patients do not respond to two or more sequential antidepressant medications and are deemed to have treatment-resistant depression (TRD) (Kubitz, Mehra, Potluri, Garg, \& Cossrow, 2013; McCall, 2007). TRD patients are candidates for electroconvulsive therapy (ECT), acknowledged as the most effective TRD treatment (Lisanby, 2007).

HRQOL is exceptionally poor in MDD patients referred for ECT and worse than that of unselected MDD patients in general outpatient settings, (McCall et al., 2013) and HRQOL is a factor in referral patterns for ECT (W. V. McCall, W. Cohen, B. Reboussin, \& P. Lawton, 1999). Naturalistic studies of MDD have shown that ECT results in improved HRQOL, with the degree of improvement greater for patients who received ECT as opposed to antidepressant medications (McCall, Reboussin, Cohen, \& Lawton, 2001). Similarly, modern ECT randomized clinical trials (RCT) not including a non-ECT comparator arm also showed improvement in HQOL (McCall, Dunn, \& Rosenquist, 2004; McCall et al., 2011). Both in the naturalistic studies and the prior RCTs, improvement in HRQOL was best explained by improvement in depression symptoms, with little or no relationship to any cognitive side effects. In naturalistic studies, improvement in HRQOL was sustained over 6-months after ECT in patients with sustained remission with HRQOL values indistinguishable from healthy population norms (McCall et al., 2013). In contrast, depressive relapse after ECT was associated with worsening in HRQOL (McCall, Prudic, Olfson, \& Sackeim, 2006).

HRQOL is central to understanding the overall net risks and benefits of treatments, including those of ECT. While ECT results in remission of depressive symptoms, it is also associated with cognitive side effects. The issue of cognitive side effects is of particular concern for the elderly population who are more vulnerable to age-related cognitive problems (Rizzi, Rosset, \& Roriz-Cruz, 2014). Medical decision-making regarding the risk/benefit ratio of ECT could be usefully informed by the study of health related quality of life measures (Devanand, Dwork, Hutchinson, Bolwig, \& Sackeim, 1994; Scalia et al., 2007; Weiner, 1984). However, prior HRQOL studies in ECT have lacked randomized comparisons of ECT versus a non-ECT alternative group.

We present here the HRQOL outcomes as a secondary analysis from a randomized

comparison of ECT combined with venlafaxine (VEN) and lithium (Li), versus VEN and Li without $E C T$, as continuation therapy after a successful ECT course for elderly adults with MDD.

\section{MATERIAL AND METHODS}

\section{Design Overview}


The Prolonging Remission in Depressed Elderly (PRIDE) study was a NIH-funded randomized, multi-center study that compared two post-acute-ECT continuation treatment strategies: (1) pharmacotherapy that combined venlafaxine (VEN) and lithium (Li) (PHARM); and (2) PHARM enhanced by the addition of an individualized, flexible, algorithm-based ECT schedule (Symptom-Titrated, Algorithm-Based, Longitudinal ECT, STABLE) (STABLE + PHARM) (Lisanby et al., 2008).

PRIDE consisted of two phases: in Phase 1, 240 patients, $\geq 60$ years old with unipolar MDD received acute ECT 3 times per week in combination with oral VEN; in Phase 2, 120 remitters who were randomized to either PHARM or STABLE + PHARM comprised the intent-to-treat (ITT) sample. The primary efficacy outcome variable was the 24-item Hamilton Rating Scale for Depression $\left(\mathrm{HRSD}_{24}\right)$ total score measured longitudinally over 6-months. A priori secondary outcome variables included HRQOL. The results of Phase 1 have been previously reported for both antidepressant efficacy and HRQOL (Kellner, Husain, Knapp, McCall, Petrides, Rudorfer, Young, Sampson, McClintock, Mueller, Prudic, Greenberg, Weiner, Bailine, Rosenquist, Raza, Kaliora, Latoussakis, Tobias, Briggs, Liebman, Geduldig, Teklehaimanot, Lisanby, et al., 2016; McCall et al., 2017) and the Phase 2 efficacy results have been reported (Kellner, Husain, Knapp, McCall, Petrides, Rudorfer, Young, Sampson, McClintock, Mueller, Prudic, Greenberg, Weiner, Bailine, Rosenquist, Raza, Kaliora, Latoussakis, Tobias, Briggs, Liebman, Geduldig, Teklehaimanot, Dooley, et al., 2016). The study was approved by the institutional review board at each study site, and the investigation was carried out in accordance with the latest version of the Declaration of Helsinki.

\section{Patient Sample}

Patients enrolled in Phase 1 were aged 60 years and older and were referred for ECT for the treatment of unipolar MDD, without dementia, with or without psychosis, with a pretreatment $\mathrm{HRSD}_{24}$ total score $\geq 21$. Exclusion criteria included: bipolar disorder, schizoaffective disorder, schizophrenia, dementia, delirium, intellectual disability, history of substance abuse in the past 6 months, or neurological conditions or active general conditions assumed to affect cognition or treatment response. Also, patients failing to respond to an adequate trial of Li+VEN or ECT in the current episode were excluded. Inclusion criteria for the randomized phase (Phase 2) were achievement of remission in Phase 1 defined as: (a) HRSD 24 total score $\leq 10$ on two consecutive ratings, and (b) $\mathrm{HRSD}_{24}$ total score did not increase $>3$ points on the second consecutive $\mathrm{HRSD}_{24}$ or remained $\leq 6$. Written informed consent was obtained before entrance to Phase 1 and before randomization in Phase 2.

\section{Treatments}

Symptom-Titrated, Algorithm-Based Longitudinal ECT (STABLE). STABLE featured an initial fixed, tapered, ECT treatment schedule followed by a symptom driven, flexible component, in addition to the same VEN + $\mathrm{Li}$ as in PHARM, and the combination is termed STABLE + PHARM. The initial fixed portion consisted of 4 ECT sessions in one month, within specified treatment windows. Treatment frequency in the subsequent flexible component (weeks 5-24) was 
determined by application of the STABLE algorithm, which prescribed 0-2 ECT treatments in a given week based on a patient's $\mathrm{HRSD}_{24}$ total scores, details of which have been previously reported (Lisanby et al., 2008).

ECT procedures. ECT was delivered with right unilateral electrode placement with a highdose, ultrabrief pulse stimulus (RUL-UBP), described in our earlier report (Kellner, Husain, Knapp, McCall, Petrides, Rudorfer, Young, Sampson, McClintock, Mueller, Prudic, Greenberg, Weiner, Bailine, Rosenquist, Raza, Kaliora, Latoussakis, Tobias, Briggs, Liebman, Geduldig, Teklehaimanot, Dooley, et al., 2016). Continuation ECT in Phase 2 was administered at the same stimulus dose as the last treatment in Phase 1.

Medication Procedures. Open-label VEN was started in Phase 1 at an initial dosage of 37.5 mg p.o., with a target dose of $225 \mathrm{mg}$ q.d. by the end of Phase 1. This dosage was continued following randomization in Phase 2. Open-label Li was started at $300 \mathrm{mg} / \mathrm{day}$ on the day of randomization in Phase 2, with a target level for most patients in the 0.4-0.6 mEq/L range. For

VEN and Li dosing/procedures were identical for the PHARM arm and the STABLE + PHARM arm, except that Li was withheld the night before ECT in the STABLE + PHARM arm. The schedule of clinic and telephone ratings was identical for both the PHARM and STABLE + PHARM arms.

\section{Assessments}

$\underline{\mathrm{HRQOL}}$

HRQOL was measured with the Medical Outcomes Study Short Form 36 (SF-36) (J. E. Ware, 2003; J. E. Ware, Jr. \& Sherbourne, 1992). The SF-36 was measured at baseline prior to acute ECT and again at the end of Phase 1/beginning of Phase 2. Thereafter, the SF-36 was measured every 4 weeks during Phase 2. SF-36 data were scored in terms of the 8 standard subscales: Physical Functioning (PF), Role Physical (RP), Bodily Pain (BP), General Health (GH), Vitality (VT), Social Functioning (SF), Role Emotional (RE), and Mental Health (MH). The score for each subscale is the weighted sum of the questions for that subscale, transformed into a 0-100 scale. Lower scores define more disability. Individual scores were then transformed into T-scores, with means of 50 and standard deviations of 10. The 8 subscales were then aggregated into the two total scores: Physical Health Factor T-score (comprised of PF, RP, BP, and GH subscales) and Mental Health Factor T-score (comprised of the VT, SF, RE, and MH subscales) following the algorithm provided by Ware, Kosinski \& Dewey, by weighing the 8 subscale scores by their respective physical and mental factor scores and summing over the physical and mental subscale scores (J. E. Ware, Kosinski, \& Dewey, 2000). In addition, there are two age- and sex-adjusted summary scores; physical component score (PCS) and mental component score (MCS).

\section{Depression severity and remission/relapse status}

Depressive symptom severity was measured using the $\mathrm{HRSD}_{24}$, and was treated as a continuous variable for the purposes of the relationship between HRQOL and depression severity 
(Hamilton, 1960). However, participants left Phase 2 of the study if they relapsed, which was defined as two consecutive $\mathrm{HRSD}_{24}$ scores $\geq 21$, or the need for psychiatric hospitalization, or becoming suicidal (Kellner, Husain, Knapp, McCall, Petrides, Rudorfer, Young, Sampson, McClintock, Mueller, Prudic, Greenberg, Weiner, Bailine, Rosenquist, Raza, Kaliora, Latoussakis, Tobias, Briggs, Liebman, Geduldig, Teklehaimanot, Dooley, et al., 2016).

\section{Cognitive Function}

The Wechsler Test of Adult Reading (WTAR) was administered once at Phase 1

baseline as an estimate of pre-morbid intellectual functioning (The Psychological Corporation, 2001). Global cognitive function was measured with the Mini Mental State Examination (MMSE)(Folstein, Folstein, \& McHugh, 1975).

The second edition of the California Verbal Learning Test (CVLT-II) was used to assess delayed recall of verbal information, expressed as “\% retention" (Delis, Kaplan, Kramer, \& Ober, 2000; Woods, Delis, Scott, Kramer, \& Holdnack, 2006).

The Dementia Rating Scale-2 ${ }^{\text {nd }}$ edition Initiation/Perseveration Index (DRS-2 I/P) was used to measure executive function (Lezak, 2004). The MMSE was measured every two weeks, CVLT-II monthly, and DRS-2 I/P was measured at midpoint and end of the study period.

\section{Randomization and Masking of Treatment Assignment}

The permuted block method of randomization was used, stratified by site. Block size was varied to minimize the likelihood of unmasking. Clinical raters and neuropsychological technicians were masked to treatment assignment.

\section{Statistical Analysis}

All statistical analyses were done by the authors (RGK, MM, and MD). Analysis was conducted on an intent-to-treat (ITT) sample using SAS 9.4. Longitudinal analyses were conducted using mixed effect models (MEM) to examine change in SF-36 sub-scores over a 24week period. Simple models were analyzed for both the 2 SF-36 component and total scores, as well as the 8 sub-scores, with the total scores/subscores as the dependent variable and with fixed effects for the independent variables treatment (STABLE vs. PHARM), time (even weeks), and the time-by-treatment interaction. Expanded models adjusted the simple models for clinical site as a stratification variable, psychosis, WTAR, and time varying effect of CVLT-II retention, DRS-2 I/P, and MMSE. All available data for these variables collected during Phase 2 were used in these models. Treatment interactions for age and psychosis were tested for significance. Additionally, expanded models for the total scores and sub-scores were adjusted for age and gender, whereas the PCS and the MCS were calculated as age- and gender-adjusted scores and therefore did not need further adjustment. Furthermore, expanded models were adjusted for the time varying effect of $\mathrm{HRSD}_{24}$, except for those sub-scores that included questions that were similar to those 
of $\mathrm{HRSD}_{24}$ (MCS, Mental Health Factor T-Score, and MH). To account for random effects of subjects, random intercepts were used in all models and random slopes were used in models where the G-matrix was positive definite.

\section{RESULTS}

120 patients were randomized to PHARM $(\mathrm{N}=59)$ and STABLE + PHARM (N=61). Patients entering into Phase 2 had a mean HRSD $_{24}$ total score of 6.1 ; the majority were female (61.7\%) and white $(95 \%)$, with a mean age of $70.5 \pm 7.2$ years. The patients assigned to STABLE + PHARM received an average of $4.5 \pm 2.5$ continuation ECT sessions during Phase 2 .

The time and group adjusted SF-36 mean score difference at 24 weeks was statistically significant between PHARM and STABLE + PHARM for PCS, MCS, total scores and all of the subscores (all $p<0.02$ ) except the BP sub-score $(p=0.078)$. Patients randomized to STABLE + PHARM had significantly higher quality of life scores at the 24-week visit compared to patients in the PHARM group. All SF-36 sub-scores as well as PCS and MCS showed a statistically significant timeby-treatment interaction in the simple models (all $p<0.04$, Table 1 ) indicating that the effect of time on quality of life depended on the treatment arm.

When the simple models were adjusted for additional covariates, all models except for the SF-36 sub-scores SF, RP, and RE resulted in statistically significant adjusted mean sub-score differences at 24 weeks (all $p<0.04$, results not shown). HRSD 24 was statistically significantly associated with SF-36 PCS and sub-scores in all models where it was included except for Physical Health Factor T-Score (Table 2). Group assignment to STABLE + PHARM versus PHARM did not significantly contribute to the model after accounting for the effects of $\mathrm{HRSD}_{24}$ (Table 2). For models that did not include $\mathrm{HRSD}_{24}$, time was statistically significantly associated with both SF36 MCS and Mental Health Factor T Score (all $p<0.02$ ) but not MH sub-score $(p=0.09)$. Age was statistically significantly associated with SF-36 sub-scores Physical Health Factor T-Score, PF, and RP (Table 2). Of particular note, cognitive variables had modest associations to HRQOL variables (Table 2).

\section{DISCUSSION}

This is the first report of the HRQOL effects in a randomized comparison of a treatment strategy that included ECT combined with medication versus medication alone. Participants who received ECT in the STABLE + PHARM group had better HRQOL on every dimension of the SF-36 across 6-months of follow-up compared with the patients in the PHARM group. The HRQOL benefits were best explained by superior control of depressive symptoms in the STABLE + PHARM group, with negligible contribution of cognitive function variables. The results of this study are especially meaningful since elderly participants with severe MDD may be viewed as a particularly vulnerable population who are referred for ECT (W. V. McCall et al., 1999; McCall \& Dunn, 2003). These results provide strong evidence that ECT produces an overall net health benefit. Strengths of this study included a well-controlled design, use of standardized clinical neuropsychological variables to assess cognitive function, use of standardized psychometrically sound measures of 
HRQOL, and an accounting of other confounding variables. However, as this study was designed to examine prevention of depressive relapse and was not designed to examine acute antidepressant effects, caution is warranted in drawing conclusions about HRQOL effects of ECT during acute treatment of depression in which the ECT treatments would be given more frequently than was done in this relapse prevention intervention.

The current report has some limitations. First, the measure of HRQOL was completed by patient self-report, and we did not include any measures of clinician-rated or third-party observers, but in prior work we showed that ECT patients' assessment of their functional status closely matched the assessments of observers (McCall, Dunn, Rosenquist, \& Hughes, 2002). Second, lithium, venlafaxine, and as-needed lorazepam were the only medications examined in this study, while RUL UB ECT was the only form of ECT. Other medication regimens and other ECT treatment approaches may have produced different results. Indeed, different electrode placements have been shown to have differential effects on HRQOL, with RUL having superior HRQOL outcomes as compared with bilateral electrode placement (Galvez et al., 2016; McCall et al., 2011). Finally, the number of ECT treatments in the STABLE arm was low in comparison to a typical acute course of ECT; however, it is remarkable that a low number of ECTs were sufficient to enhance HRQOL over a 6-month period.

The present report adds to the literature on the beneficial effects of ECT on HRQOL and the overall net health benefits. Our findings should assure patients, families and caregivers of depressed elderly patients that ECT is a medically appropriate choice for elderly adults with MDD and is likely to produce improvement in $\mathrm{HRQOL}$ that can be sustained during the continuation phase of treatment by employing combined ECT and medication to support remission. The results of the PRIDE study support the merit of continuing ECT as a means of staving off depressive symptoms. Our findings substantiate that for elderly adults with MDD, ECT can be a quality-oflife-enhancing treatment when used as a continuation strategy in combination with medication to prolong remission. 


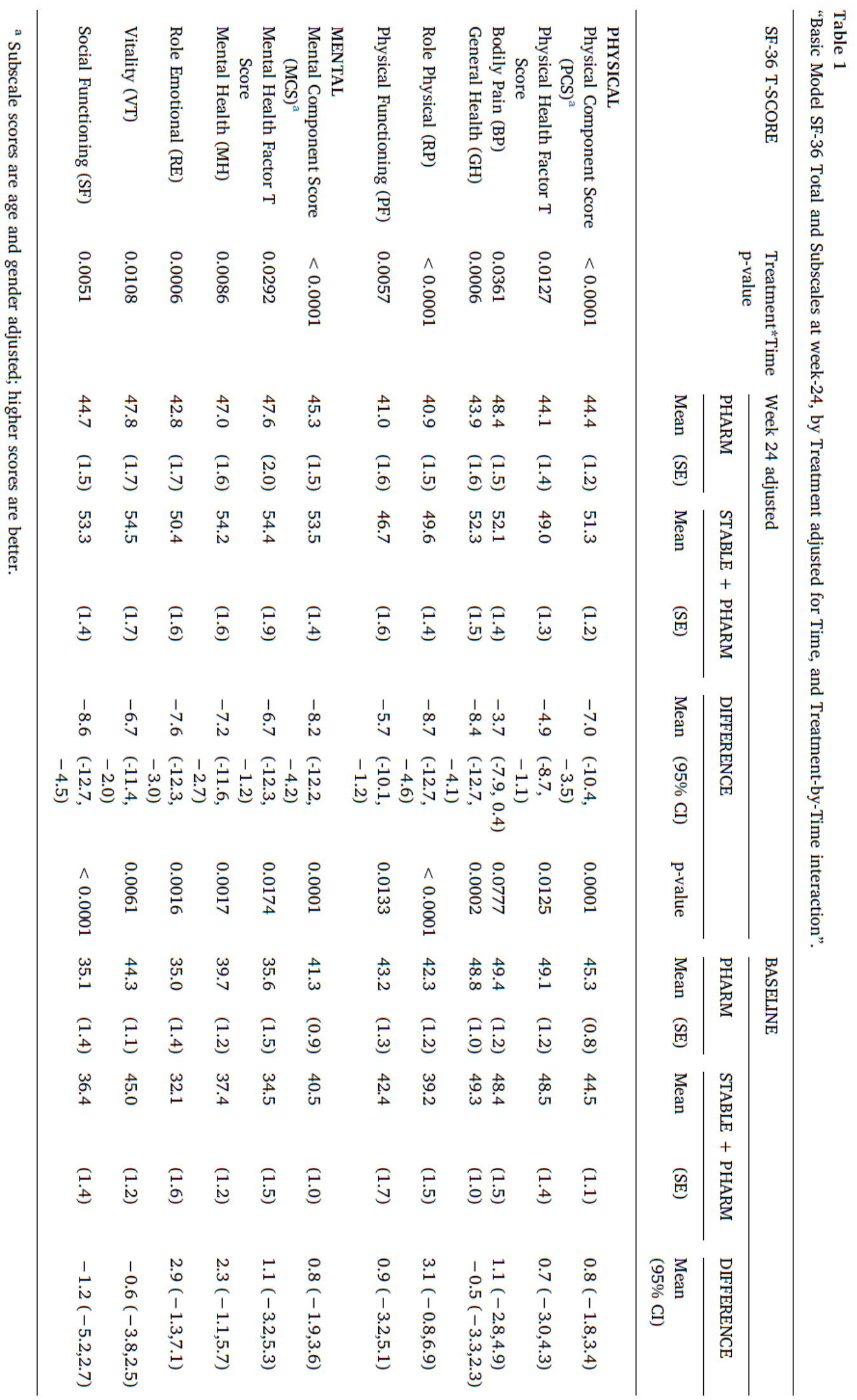




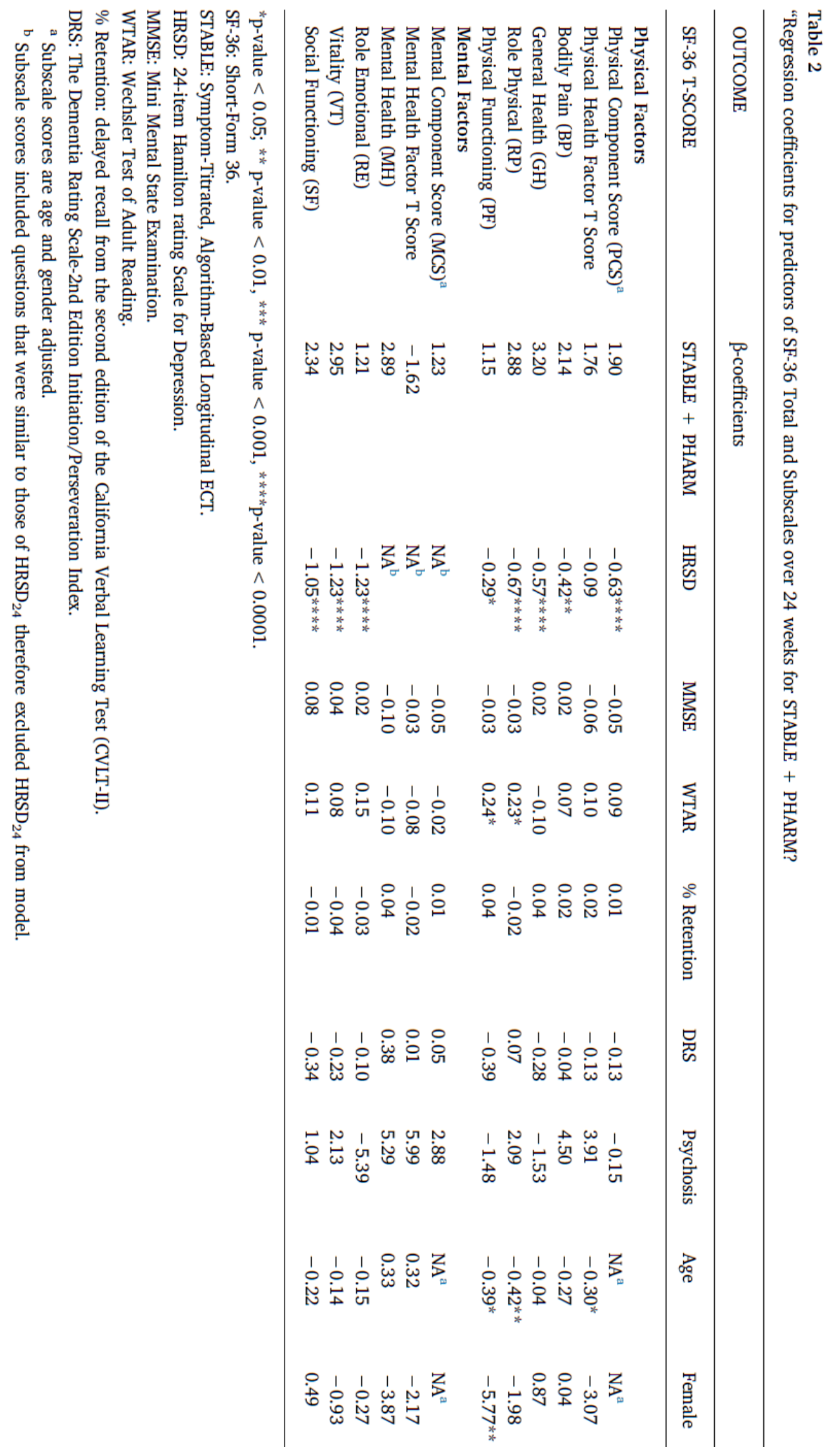


Funding: Supported by NIMH grant U01MH055495-09 (Mt Sinai); U01MH055495-09., 1U01MH086127-01 (Wake Forest); 7U01MH086127 and U01MH086127 (Medical College of Georgia); U01MH081362 (Medical University of South Carolina); 5U01-MH086122 (Weill Cornell Medicine ); 5U01MH84241-5 (Duke); 5U01MH086130 (Mayo); 5U01MH08612005 (UTSW); U01 MH084241 (Duke); U01-MH086123 (LIJ)

Acknowledgements: The authors thank the following persons for their assistance in collecting data and for this study: Ahmad Raza, M.D., Styliani Kaliora, M.D., Vassilios Latoussakis, M.D., Kristen G. Tobias, M.A., Mimi C. Briggs, B.A., Lauren S. Liebman, B.A., Emma T. Geduldig, B.A., Abeba Teklehaimanot, M.S., and Niki Boggs.

Role of the Funding Source: The NIMH played no role in the collection, management, analysis, and interpretation of the data, or preparation, review, or the decision to submit the manuscript for publication, but did approve design and conduct of the study by supplying a NIMH DSMB. The NIMH also approved the final version on behalf of Dr. Lisanby and Dr. Rudorfer.

Drs. Knapp and Mueller had full access to all the data in the study and take responsibility for the integrity of the data and the accuracy of the data analysis. 
Youssef's contributions (added for the purpose of this thesis): Nagy Youssef was a coinvestigator at the Duke University site for the PRIDE study and one of the study's ECT psychiatrists. His role as an investigator and psychiatrist included reviewing labs and records, interviewing and recruiting patients, making recruiting designs, explaining the study to participants and obtaining informed consents, and performing ECT as 1 of 3 ECT psychiatrists. He also supervised the research assistants and followed the patients with the research assistants during stage I and II of the clinical trial. His effort and salary support on the NIH grant included $50 \%$ effort. 


\section{REFERENCES}

Delis, D., Kaplan, E., Kramer, J., \& Ober, B. (2000). California Verbal Learning Test-Second Edition (CVLT-II). San Antonio, TX: The Psychological Corporation.

Devanand, D. P., Dwork, A. J., Hutchinson, E. R., Bolwig, T. G., \& Sackeim, H. A. (1994). Does ECT alter brain structure? Am J Psychiatry, 151(7), 957-970. doi:10.1176/ajp.151.7.957

Folstein, M. F., Folstein, S. E., \& McHugh, P. R. (1975). "Mini-mental state". A practical method for grading the cognitive state of patients for the clinician. J Psychiatr Res, 12(3), 189198. doi:10.1016/0022-3956(75)90026-6

Galvez, V., Li, A., Oxley, C., Waite, S., De Felice, N., Hadzi-Pavlovic, D., . . . Loo, C. K. (2016). Health Related Quality of Life after ECT for depression: a study exploring the role of different electrode-placements and pulse-widths. J Affect Disord, 206, 268-272. doi:10.1016/j.jad.2016.08.002

Hamilton, M. (1960). A rating scale for depression. J Neurol Neurosurg Psychiatry, 23, 56-62. doi:10.1136/jnnp.23.1.56

Kellner, C. H., Husain, M. M., Knapp, R. G., McCall, W. V., Petrides, G., Rudorfer, M. V., . . . Group, C. P. W. (2016). A novel strategy for continuation ECT in geriatric depression: phase 2 of the PRIDE Study. Am J Psychiatry, 173(11), 1110-1118. doi:10.1176/appi.ajp.2016.16010118

Kellner, C. H., Husain, M. M., Knapp, R. G., McCall, W. V., Petrides, G., Rudorfer, M. V., . . . Group, C. P. W. (2016). Right unilateral ultrabrief pulse ECT in geriatric depression: phase 1 of the PRIDE Study. Am J Psychiatry, 173(11), 1101-1109. doi:10.1176/appi.ajp.2016.15081101

Kubitz, N., Mehra, M., Potluri, R. C., Garg, N., \& Cossrow, N. (2013). Characterization of treatment resistant depression episodes in a cohort of patients from a US commercial claims database. PloS one, 8(10), e76882. doi:10.1371/journal.pone.0076882

Lezak, M. D. (2004). Neuropsychological assessment (4th ed.). New York: Oxford University Press.

Lisanby, S. H. (2007). Electroconvulsive therapy for depression. N Engl J Med, 357(19), 19391945. doi:10.1056/NEJMct075234

Lisanby, S. H., Sampson, S., Husain, M. M., Petrides, G., Knapp, R. G., McCall, W. V., ... Kellner, C. H. (2008). Toward individualized post-electroconvulsive therapy care: piloting the Symptom-Titrated, Algorithm-Based Longitudinal ECT (STABLE) intervention. J ect, 24(3), 179-182. doi:10.1097/YCT.0b013e318185fa6b

McCall, W. V. (2007). What does Star*D tell us about ECT? Journal of ECT, 23(1), 1. doi:10.1097/01.yct.0000263249.83549.34

McCall, W. V., Cohen, W., Reboussin, B., \& Lawton, P. (1999). Effects of mood and age on quality of life in depressed inpatients. J Affect Disord, 55(2), 107-114.

doi:10.1016/S0165-0327(98)00204-3 
McCall, W. V., Cohen, W., Reboussin, B., \& Lawton, P. (1999). Pretreatment differences in specific symptoms and quality of life among depressed inpatients who do and do not receive electroconvulsive therapy: a hypothesis regarding why the elderly are more likely to receive ECT. J ect, 15(3), 193-201. Retrieved from http://www.ncbi.nlm.nih.gov/pubmed/10492857

McCall, W. V., Dunn, A., \& Rosenquist, P. B. (2004). Quality of life and function after electroconvulsive therapy. Br J Psychiatry, 185, 405-409. doi:10.1192/bjp.185.5.405

McCall, W. V., \& Dunn, A. G. (2003). Cognitive deficits are associated with functional impairment in severely depressed patients. Psychiatry Res, 121(2), 179-184. doi:10.1016/j.psychres.2003.09.003

McCall, W. V., Dunn, A. G., Rosenquist, P., \& Hughes, D. (2002). Proxy validation of patient selfreports of $A D L$ and IADL function before and after electroconvulsive therapy. $J$ ect, 18(2), 74-79. Retrieved from http://www.ncbi.nlm.nih.gov/pubmed/12195134

McCall, W. V., Lisanby, S. H., Rosenquist, P. B., Dooley, M., Husain, M. M., Knapp, R. G., . . . CORE/PRIDE Work Group. (2017). Effects of a right unilateral ultrabrief pulse electroconvulsive therapy course on health related quality of life in elderly depressed patients. J Affect Disord, 209, 39-45. doi:10.1016/j.jad.2016.11.003

McCall, W. V., Prudic, J., Olfson, M., \& Sackeim, H. (2006). Health-related quality of life following ECT in a large community sample. J Affect Disord, 90(2-3), 269-274. doi:10.1016/j.jad.2005.12.002

McCall, W. V., Reboussin, B. A., Cohen, W., \& Lawton, P. (2001). Electroconvulsive therapy is associated with superior symptomatic and functional change in depressed patients after psychiatric hospitalization. J Affect Disord, 63(1-3), 17-25. doi:10.1016/S01650327(00)00167-1

McCall, W. V., Reboussin, D., Prudic, J., Haskett, R. F., Isenberg, K., Olfson, M., . . Sackeim, H. A. (2013). Poor health-related quality of life prior to ECT in depressed patients normalizes with sustained remission after ECT. J Affect Disord, 147(1-3), 107-111. doi:10.1016/j.jad.2012.10.018

McCall, W. V., Rosenquist, P. B., Kimball, J., Haskett, R., Isenberg, K., Prudic, J., . . Sackeim, H. A. (2011). Health-related quality of life in a clinical trial of ECT followed by continuation pharmacotherapy: effects immediately after ECT and at 24 weeks. $J$ ect, 27(2), 97-102. doi:10.1097/YCT.0b013e318205c7d7

Rizzi, L., Rosset, I., \& Roriz-Cruz, M. (2014). Global Epidemiology of dementia: Alzheimer's and vascular types. BioMed Research International, 2014, 908915. doi:10.1155/2014/908915

Scalia, J., Lisanby, S. H., Dwork, A. J., Johnson, J. E., Bernhardt, E. R., Arango, V., \& McCall, W. V. (2007). Neuropathologic examination after $91 \mathrm{ECT}$ treatments in a 92-year-old woman with late-onset depression. J ect, 23(2), 96-98. doi:10.1097/YCT.0b013e31804bb99d

The Psychological Corporation. (2001). Wechsler test of adult reading. San Antonio, TX: Harcourt Assessment. 
Ware, J. E. (2003). SF-36 health survey: manual and interpretation guide. Lincoln, RI: QualityMetric Inc.

Ware, J. E., Jr., \& Sherbourne, C. D. (1992). The MOS 36-item short-form health survey (SF-36). I. Conceptual framework and item selection. Med Care, 30(6), 473-483. Retrieved from http://www.ncbi.nlm.nih.gov/pubmed/1593914

Ware, J. E., Kosinski, M., \& Dewey, J. E. (2000). How to score version 2 of the SF-36 health survey: standard \& acute forms (3rd ed. ed.). Lincoln, RI: QualityMetric Inc.

Weiner, R. D. (1984). Does electroconvulsive therapy cause brain damage? Behavioral and Brain Sciences, 7(1), 1-53. doi:10.1017/S0140525X00025887

Woods, S. P., Delis, D. C., Scott, J. C., Kramer, J. H., \& Holdnack, J. A. (2006). The California Verbal Learning Test - second edition: Test-retest reliability, practice effects, and reliable change indices for the standard and alternate forms. Archives of Clinical Neuropsychology, 21(5), 413-420. doi:10.1016/j.acn.2006.06.002

World Health Organization. (2011). World report on disability 2011. Retrieved from Geneva: https://www.ncbi.nlm.nih.gov/books/NBK304079/ 



\section{Chapter 10 Charting The ECT Course: Where Have We Been? Where}

Are We Headed?

Peter B. Rosenquist, $\mathrm{MD}^{1}$, W. Vaughn McCall MD, MS ${ }^{1}$, Nagy Youssef, MD ${ }^{1}$

${ }^{1}$ Department of Psychiatry \& Health Behavior, Medical College of Georgia at Augusta University, Augusta, GA 30912, USA

2 Academic Affairs, Medical College of Georgia, Augusta University, Augusta, GA 30912, USA

Published as: Rosenquist PB, McCall WV, Youssef N. Charting the Course of Electroconvulsive Therapy: Where Have We Been and Where Are We Headed? J Psychosoc Nurs Ment Health Serv. 2016 Dec 1;54(12):39-43. doi: 10.3928/02793695-20161208-08. PubMed PMID:28001286. https://www.healio.com/psychiatry/journals/ipn/2016-12-54-12/\%7B93c614cf-bcc0-4e4797ab-ead763c4a9fe\%7D/charting-the-course-of-electroconvulsive-therapy-where-have-webeen-and-where-are-we-headed 


\begin{abstract}
Electroconvulsive Therapy (ECT) is one of the oldest and arguably remains the gold standard treatment for severe mental illness in psychiatry. A safe and highly effective option for treatment-resistant mood disorders, ECT is positively life-saving for those suffering from catatonia and acute suicidality. Less recognized are ECT's benefits in the treatment of primary psychotic disorders, Parkinson's disease and status epilepticus. In the past decade, evidence from multi-site clinical trials highlights an evolving standard for the delivery of ECT to achieve and maintain remission and quality of life. Today, the optimal practice of ECT is defined by evidencebased treatment planning including patient selection, choice of electrode placement and stimulus parameters, augmentation with pharmacotherapy, and the use of continuation/maintenance treatment. Research into biomarkers and neuroplasticity related to ECT response, as well as new, investigational methods of delivering ECT provide a glimpse into the future of this time-honored treatment.
\end{abstract}




\section{INTRODUCTION}

ECT for severe mental illness has withstood the test of time, despite persistent stigma based upon the perpetuation of an inaccurate and antiquated view of the procedure. Patients and caregivers ask, "Shock therapy is still being used today?" The answer is definitively yes--but today's ECT is dynamic and steadily improving. In this review, we trace changes in technique emerging from treatment-oriented research through the past several decades. Even as this work has produced demonstrable improvements, many unanswered questions point the way forward.

\section{PATIENT SELECTION AND OUTCOME}

Every treatment may be characterized by range of efficacy, durability, dose-response and dose-toxicity relationships. Patient selection is the first principle of optimization. Remarkably broad spectrum in range of efficacy, strong evidence supports the use of ECT for mood disorders-both major depressive (MDD) and bipolar disorder (Bailine et al., 2010). Catatonia, now recognized in DSM-5 as a separate syndrome occurring in conjunction with a number of other manifestations of brain pathology, is one indication for which ECT may be uniquely beneficial (Luchini et al., 2015). Similarly, ECT should be considered "first-line" in the treatment of mood disorders with psychotic features because of its rapid onset and effectiveness (Petrides et al., 2001). Other presentations for which ECT is appropriately elevated in the treatment algorithm include acute/high-risk suicidal ideation and behavior (Fink, Kellner, \& McCall, 2014)late-life depression (Dombrovski \& Mulsant, 2007), and Parkinson-plus syndromes where ECT has demonstrated benefits on motor and non-motor symptoms (Narang, Glowacki, \& Lippmann, 2015). While still seen as "last resort," ECT successfully aborts treatment-resistant status epilepticus in up to $80 \%$ of reported cases (Lambrecq et al., 2012).

Less recognized are the benefits in the treatment of primary psychotic disorders as well as disturbances of mood and thought resulting from neurologic or substance-related causes. A curious divergence in the worldwide use of ECT has occurred with schizophrenia emerging as the primary indication for ECT in Asia, as opposed to mood disorders in the West. While the reasons for this are not entirely clear, the evidence base has lagged somewhat for psychotic disorders. Recent work has demonstrated additive effects of antipsychotic medication therapy when combined with ECT in treatment-resistant schizophrenia (Wang et al., 2015). Of those dimensions of psychotic disorders currently identified in DSM-5, ECT appears to be most beneficial for catatonic, positive and mood symptoms, and to a lesser extent cognition, negative symptoms, and thought disorder, although the mechanism of its broad spectrum of effects is less clear (Rosenquist, Miller, \& Pillai, 2014).

While treatment resistance often results in referral for ECT, it should be understood that this may diminish expectation of efficacy for MDD (Haq, Sitzmann, Goldman, Maixner, \& Mickey, 2015). Nevertheless, patients who have failed to respond to multiple medication trials may be more likely to benefit from ECT compared with another round of medication. The presence of a comorbid personality disorder appears to have a negative influence on the outcome of ECT as does duration of illness, suggesting that ECT is most beneficial for more acute and/or episodic neuropathological states (Rasmussen, 2015; Rasmussen, Hart, \& Lineberry, 2008). However, ECT 
practitioners frequently encounter treatment-refractory patients for whom the administration of ECT results in symptomatic improvement whereupon other forms of treatment including psychotherapy became more viable.

The critics of ECT have charged that ECT is associated with a net loss of health, but the literature on quality of life (QOL) and function presents a very different picture. QOL measures dimensions such as freedom from pain, ability to enjoy relationships, managing one's own life, etc. Patients with major depressive disorder (MDD) have poor QOL compared with ambulatory primary care patients without MDD; those referred for ECT have even worse QOL. ECT confers immediate improvements in mean QOL and function in $80-90 \%$ of MDD patients, which are sustained for 6 months (McCall et al., 2013). Importantly, the improvement in QOL is explained by improvements in mood, while the patient's cognitive status after ECT does not contribute to the variance in QOL. ECT is a safe procedure, but increasing age and preexistent cardiac disease increase the risk of minor and major complications. A recent veteran's affairs study showed no deaths associated with ECT in any VA hospital between 1999 and 2010. Based on the number of treatments given, the authors estimate the mortality rate associated with ECT as less than 1 death per 73,440 treatments, with the most common adverse events reported being injury to the mouth (including dental and tongue injury) and problems related to paralysis (Watts, Groft, Bagian, \& Mills, 2011).

\section{TREATMENT COURSE}

The question frequently arises, "how many ECT sessions are required?" A standard answer might be 6-12 treatments in a so-called "index" acute course administered 2-3 times weekly until the patient remits, defined by a rating scale score using a clinician or patient selfrated instrument validated for this purpose (Hamilton, 1960; Rush et al., 2006). The use of such measures ensures that patient and provider are cognizant of gradual improvements which lag behind global assessments prejudiced by depressive cognitive bias. Speed of response is variable, with some patients noting rapid cessation of suicide ideation or achieving full remission within a few treatments. Longer treatment courses are generally expected in order to achieve meaningful improvements in symptoms of schizophrenia.

\section{DOSING}

One might ask why not optimize treatment by using the highest dosage from the outset of treatment? Like drug treatment, ECT is associated with dose-dependent cognitive side effects-post-ictal impairments in attention, concentration and deficits in autobiographical memory. These develop over the course of treatment and can often be mitigated by a step-wise approach. Many practitioners have adopted a method of dose-titration, thereby identifying an individualized seizure threshold defined by the lowest electrical charge required to initiate a seizure in a particular patient. This forms the basis for dosing subsequent treatment. Typically, bitemporal (BT) and bifrontal (BF) treatment is delivered at 1.5 to 2 times the seizure threshold, whereas right unilateral (RUL) treatment requires 4-6 times the seizure threshold in order to achieve a positive treatment outcome (Kellner et al., 2010). 


\section{Figure. Electrode Placement and Stimulus Characteristics}

Figure 1

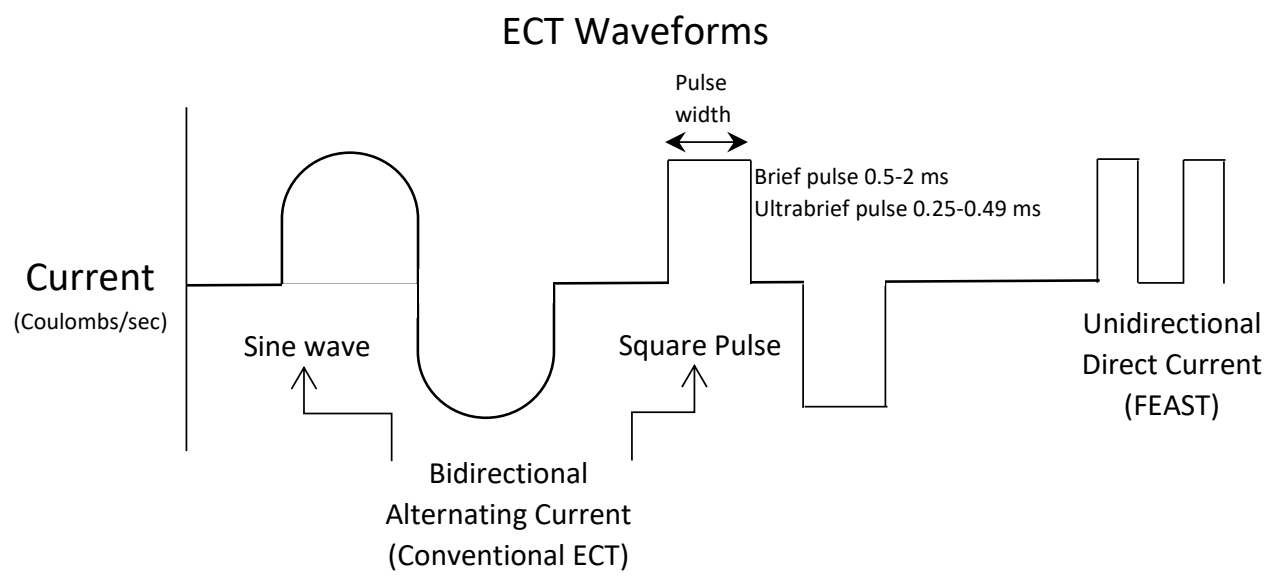

Initial choice of electrode placement is subject to some interpretation. Compared with RUL and BF, BT ECT is associated with significantly greater risk of cognitive impairment (Dunne \& McLoughlin, 2012; Sackeim et al., 2007). Yet, many ECT practitioners default to BT treatments based upon the perception of more robust or more rapid response. However, the body of evidence in support of RUL ECT suggests that crossover to BT is required in a minority of cases.

The nature of the ECT stimulus has evolved over time (Figure 1). Though effective at seizure generation, sine wave ECT was shown to be definitively more cognitively impairing and is now considered outdated (Sackeim et al., 2007). Modern ECT devices deliver a rectified "square" pulse. The square pulse is more efficient and therefore requires less total charge to induce a seizure. Similarly, decreasing the pulse width ( 2 milliseconds versus 1 millisecond) is more efficient and less likely to produce cognitive impairment. "Brief" pulses of 0.5 to $1 \mathrm{~ms}$ are generally effective and well tolerated. More recently "ultra-brief" pulse ECT defined as less than $0.5 \mathrm{~ms}$ (commonly 0.25 to $.37 \mathrm{~ms}$ with current devices) have appeared to be still more efficient and cognitively benign. For unclear reasons, the initial study of ultra-brief stimuli, ultra-brief BT ECT was less effective than all other arms in the study (brief BT, brief and ultra-brief RUL) (Sackeim et al., 2008). While confirming the relative cognitive advantage of ultra-brief RUL, other studies have produced conflicting results about efficacy. Some studies show lower rates of remission or prolonged treatment course, but efficacy appears to be comparable at a higher stimulus intensity (8 times seizure threshold) (Loo et al., 2014).

\section{COORDINATION WITH OTHER THERAPIES TO ACHIEVE AND MAINTAIN REMISSION}

ECT is generally not employed as monotherapy. One important discovery was that the remission rate for MDD is improved with concomitant antidepressant medication therapy. ECT plus placebo resulted in a remission rate of only $49 \%$, whereas the addition of venlafaxine or 
nortriptyline improved this to $62 \%$ and $64 \%$ respectively (Sackeim et al., 2009). A lack of antidepressant treatment following remission with ECT results in relapse for $90 \%$ of patients, whereas maintenance pharmacotherapy with antidepressant alone is only marginally effective (61\% relapse within 6 months) (Sackeim et al., 2001). Augmentation of antidepressants with lithium is the gold standard for pharmacotherapy following ECT, reducing relapse rates to only 240\% (Nordenskjold et al., 2013; Sackeim et al., 2001).

There is emerging evidence that the addition of maintenance ECT might be more helpful in treatment-resistant cases to maximize chances of sustaining remission (Youssef \& McCall, 2014). In a large randomized trial, maintenance ECT alone was demonstrated to be equivalent to lithium plus nortriptyline (sustained remission rate of 46\%) (Kellner et al., 2006). Additional studies have shown that maintenance ECT plus pharmacotherapy is more effective than pharmacotherapy alone at one and two year follow-up (Navarro et al., 2008).

\section{BIOMARKERS}

There are no widely accepted biomarkers of clinical response, though a number of studies have shown that electroencephalographic measures of induced seizure duration or morphology are modest predictors. These so-called "seizure quality" measures may be identified by visual inspection or by algorithms built into the ECT device. When the patient is improving as expected, such measures are of little consequence. However, when the patient is slow to respond, seizure quality measures may provide additional impetus to increase stimulus dose, change electrode placement or withdraw concomitant medications in order to potentially improve the seizure quality.

Preliminary studies in humans and animals show favorable effect of ECT in inducing neurogenesis and neuroplasticity. One study demonstrated increased hippocampal and amygdala volumes after ECT which correlated with an improved clinical response (Joshi et al., 2016). These data have attracted attention to the possible role of adult neurogenesis in the antidepressant effect of pharmacological agents (Rotheneichner et al., 2014).

\section{THE FUTURE OF ECT}

The future of ECT depends upon continued refinement of the technique to minimize side effect burden while maintaining effectiveness. Two lines of innovation are currently being investigated with the goal of diminishing side effects by making seizure activity more focalmagnetic seizure therapy (MST) and focal electrically-administered seizure therapy (FEAST). MST capitalizes on the fact that the skull is transparent to the magnetic field and easily overcomes scalp/skull impedance. The field is transferred in the brain by Eddy currents (Faraday's law of induction) with more focality (Lisanby, Luber, Schlaepfer, \& Sackeim, 2003). FEAST uses a unidirectional-pulsed stimulus along with asymmetrically-sized electrodes (Nahas et al., 2013) to deliver a focal current to the target areas believed to be most responsible for depression, including the right orbitofrontal cortex. 
Preliminary research suggests that FEAST and MST are cognition-sparing, compared to traditional ECT but have yet to demonstrate comparable efficacy. MST presents unique engineering challenges because of inefficient energy transfer to brain tissue requiring excessively high-powered devices to generate a seizure. Also, the penetration in the brain is superficial with a depth of only 2-4 cm below the coil (Deng, Lisanby, \& Peterchev, 2011).

While we envision that ECT will remain the backbone of treatment for severe and treatment-resistant psychiatric illness, mounting experience with alternative brain stimulation modalities such as repetitive transcranial magnetic stimulation, and transcranial direct current stimulation may well provide novel insights to guide the further development of this timehonored treatment. 
Contributions on this manuscript for the purpose of this thesis: Nagy Youssef searched the literature, wrote about a third of the paper, as the coauthors divided the sections to be written, and all coauthors edited the interim versions of the paper as well as the final paper. Other authors did similar effort and wrote the rest of the manuscript. In addition, Peter Rosenquist, the first author, packaged the final manuscript and responded to reviewers' suggestions. 


\section{REFERENCES}

Bailine, S., Fink, M., Knapp, R., Petrides, G., Husain, M. M., Rasmussen, K., . . Kellner, C. H. (2010). Electroconvulsive therapy is equally effective in unipolar and bipolar depression. Acta Psychiatr Scand, 121(6), 431-436. doi:10.1111/j.1600-0447.2009.01493.x

Deng, Z. D., Lisanby, S. H., \& Peterchev, A. V. (2011). Electric field strength and focality in electroconvulsive therapy and magnetic seizure therapy: a finite element simulation study. J Neural Eng, 8(1), 016007. doi:10.1088/1741-2560/8/1/016007

Dombrovski, A. Y., \& Mulsant, B. H. (2007). The evidence for electroconvulsive therapy (ECT) in the treatment of severe late-life depression. ECT: the preferred treatment for severe depression in late life. Int Psychogeriatr, 19(1), 10-14, 27-35; discussion 24-16. doi:10.1017/S1041610206224384

Dunne, R. A., \& McLoughlin, D. M. (2012). Systematic review and meta-analysis of bifrontal electroconvulsive therapy versus bilateral and unilateral electroconvulsive therapy in depression. World J Biol Psychiatry, 13(4), 248-258. doi:10.3109/15622975.2011.615863

Fink, M., Kellner, C. H., \& McCall, W. V. (2014). The role of ECT in suicide prevention. J ect, 30(1), 5-9. doi:10.1097/YCT.0b013e3182a6ad0d

Hamilton, M. (1960). A rating scale for depression. J Neurol Neurosurg Psychiatry, 23, 56-62. doi:10.1136/jnnp.23.1.56

Haq, A. U., Sitzmann, A. F., Goldman, M. L., Maixner, D. F., \& Mickey, B. J. (2015). Response of depression to electroconvulsive therapy: a meta-analysis of clinical predictors. $J$ Clin Psychiatry, 76(10), 1374-1384. doi:10.4088/JCP.14r09528

Joshi, S. H., Espinoza, R. T., Pirnia, T., Shi, J., Wang, Y., Ayers, B., . . Narr, K. L. (2016). Structural plasticity of the hippocampus and amygdala induced by electroconvulsive therapy in major depression. Biol Psychiatry, 79(4), 282-292. doi:10.1016/j.biopsych.2015.02.029

Kellner, C. H., Knapp, R., Husain, M. M., Rasmussen, K., Sampson, S., Cullum, M., . . Petrides, G. (2010). Bifrontal, bitemporal and right unilateral electrode placement in ECT: randomised trial. Br J Psychiatry, 196(3), 226-234. doi:10.1192/bjp.bp.109.066183

Kellner, C. H., Knapp, R. G., Petrides, G., Rummans, T. A., Husain, M. M., Rasmussen, K., ... Fink, M. (2006). Continuation electroconvulsive therapy vs pharmacotherapy for relapse prevention in major depression: a multisite study from the Consortium for Research in Electroconvulsive Therapy (CORE). Arch Gen Psychiatry, 63(12), 1337-1344. doi:10.1001/archpsyc.63.12.1337

Lambrecq, V., Villega, F., Marchal, C., Michel, V., Guehl, D., Rotge, J. Y., \& Burbaud, P. (2012). Refractory status epilepticus: electroconvulsive therapy as a possible therapeutic strategy. Seizure, 21(9), 661-664. doi:10.1016/j.seizure.2012.07.010

Lisanby, S. H., Luber, B., Schlaepfer, T. E., \& Sackeim, H. A. (2003). Safety and feasibility of magnetic seizure therapy (MST) in major depression: randomized within-subject 
comparison with electroconvulsive therapy. Neuropsychopharmacology, 28(10), 18521865. doi:10.1038/sj.npp.1300229

Loo, C. K., Katalinic, N., Smith, D. J., Ingram, A., Dowling, N., Martin, D., . . Schweitzer, I. (2014). A randomized controlled trial of brief and ultrabrief pulse right unilateral electroconvulsive therapy. Int J Neuropsychopharmacol, 18(1). doi:10.1093/ijnp/pyu045

Luchini, F., Medda, P., Mariani, M. G., Mauri, M., Toni, C., \& Perugi, G. (2015). Electroconvulsive therapy in catatonic patients: efficacy and predictors of response. World J Psychiatry, 5(2), 182-192. doi:10.5498/wjp.v5.i2.182

McCall, W. V., Reboussin, D., Prudic, J., Haskett, R. F., Isenberg, K., Olfson, M., . . Sackeim, H. A. (2013). Poor health-related quality of life prior to ECT in depressed patients normalizes with sustained remission after ECT. J Affect Disord, 147(1-3), 107-111. doi:10.1016/j.jad.2012.10.018

Nahas, Z., Short, B., Burns, C., Archer, M., Schmidt, M., Prudic, J., . . Sackeim, H. A. (2013). A feasibility study of a new method for electrically producing seizures in man: focal electrically administered seizure therapy [FEAST]. Brain Stimul, 6(3), 403-408. doi:10.1016/j.brs.2013.03.004

Narang, P., Glowacki, A., \& Lippmann, S. (2015). Electroconvulsive therapy intervention for Parkinson's disease. Innov Clin Neurosci, 12(9-10), 25-28. Retrieved from http://www.ncbi.nlm.nih.gov/pubmed/26634178

Navarro, V., Gasto, C., Torres, X., Masana, G., Penades, R., Guarch, J., . . Catalan, R. (2008). Continuation/maintenance treatment with nortriptyline versus combined nortriptyline and ECT in late-life psychotic depression: a two-year randomized study. Am J Geriatr Psychiatry, 16(6), 498-505. doi:10.1097/JGP.0b013e318170a6fa

Nordenskjold, A., von Knorring, L., Ljung, T., Carlborg, A., Brus, O., \& Engstrom, I. (2013). Continuation electroconvulsive therapy with pharmacotherapy versus pharmacotherapy alone for prevention of relapse of depression: a randomized controlled trial. J ect, 29(2), 86-92. doi:10.1097/YCT.0b013e318276591f

Petrides, G., Fink, M., Husain, M. M., Knapp, R. G., Rush, A. J., Mueller, M., . . Kellner, C. H. (2001). ECT remission rates in psychotic versus nonpsychotic depressed patients: a report from CORE. J ect, 17(4), 244-253. Retrieved from http://www.ncbi.nlm.nih.gov/pubmed/11731725

Rasmussen, K. G. (2015). Do patients with personality disorders respond differentially to electroconvulsive therapy? A review of the literature and consideration of conceptual issues. J ect, 31(1), 6-12. doi:10.1097/YCT.0000000000000165

Rasmussen, K. G., Hart, D. A., \& Lineberry, T. W. (2008). ECT in patients with psychopathology related to acute neurologic illness. Psychosomatics, 49(1), 67-72. doi:10.1176/appi.psy.49.1.67

Rosenquist, P. B., Miller, B., \& Pillai, A. (2014). The antipsychotic effects of ECT: a review of possible mechanisms. J ect, 30(2), 125-131. doi:10.1097/YCT.0000000000000131 
Rotheneichner, P., Lange, S., O'Sullivan, A., Marschallinger, J., Zaunmair, P., Geretsegger, C., . . . Couillard-Despres, S. (2014). Hippocampal neurogenesis and antidepressive therapy: shocking relations. Neural Plast, 2014, 723915. doi:10.1155/2014/723915

Rush, A. J., Bernstein, I. H., Trivedi, M. H., Carmody, T. J., Wisniewski, S., Mundt, J. C., ... Fava, M. (2006). An evaluation of the quick inventory of depressive symptomatology and the Hamilton Rating Scale for depression: a sequenced treatment alternatives to relieve depression trial report. Biol Psychiatry, 59(6), 493-501. doi:10.1016/j.biopsych.2005.08.022

Sackeim, H. A., Dillingham, E. M., Prudic, J., Cooper, T., McCall, W. V., Rosenquist, P., ... Haskett, R. F. (2009). Effect of concomitant pharmacotherapy on electroconvulsive therapy outcomes: short-term efficacy and adverse effects. Arch Gen Psychiatry, 66(7), 729-737. doi:10.1001/archgenpsychiatry.2009.75

Sackeim, H. A., Haskett, R. F., Mulsant, B. H., Thase, M. E., Mann, J. J., Pettinati, H. M., . . . Prudic, J. (2001). Continuation pharmacotherapy in the prevention of relapse following electroconvulsive therapy: a randomized controlled trial. Jama, 285(10), 1299-1307. doi:10.1001/jama.285.10.1299

Sackeim, H. A., Prudic, J., Fuller, R., Keilp, J., Lavori, P. W., \& Olfson, M. (2007). The cognitive effects of electroconvulsive therapy in community settings. Neuropsychopharmacology, 32(1), 244-254. doi:10.1038/sj.npp.1301180

Sackeim, H. A., Prudic, J., Nobler, M. S., Fitzsimons, L., Lisanby, S. H., Payne, N., . . Devanand, D. P. (2008). Effects of pulse width and electrode placement on the efficacy and cognitive effects of electroconvulsive therapy. Brain Stimul, 1(2), 71-83. doi:10.1016/j.brs.2008.03.001

Wang, W., Pu, C., Jiang, J., Cao, X., Wang, J., Zhao, M., \& Li, C. (2015). Efficacy and safety of treating patients with refractory schizophrenia with antipsychotic medication and adjunctive electroconvulsive therapy: a systematic review and meta-analysis. Shanghai Arch Psychiatry, 27(4), 206-219. doi:10.11919/j.issn.1002-0829.215093

Watts, B. V., Groft, A., Bagian, J. P., \& Mills, P. D. (2011). An examination of mortality and other adverse events related to electroconvulsive therapy using a national adverse event report system. J ect, 27(2), 105-108. doi:10.1097/YCT.0b013e3181f6d17f

Youssef, N. A., \& McCall, W. V. (2014). Relapse prevention after index electroconvulsive therapy in treatment-resistant depression. Ann Clin Psychiatry, 26(4), 288-296. Retrieved from http://www.ncbi.nlm.nih.gov/pubmed/25401716 


\section{Chapter 11 The Role of ECT in Posttraumatic Stress Disorder: A Systematic Review}

Nagy A. Youssef, MD¹; W. Vaughn McCall, MD, MS; Chittaranjan Andrade, $\mathrm{MD}^{2}$

${ }^{1}$ Department of Psychiatry and Health Behavior, The Medical College of Georgia, Augusta University, Augusta, Georgia, USA.

${ }^{2}$ Department of Psychopharmacology, National Institute of Mental Health and Neurosciences, Bangalore, India.

Published as: Youssef NA, McCall WV, Andrade C. The role of ECT in posttraumatic stress disorder: A systematic review. Ann Clin Psychiatry. 2017 Feb;29(1):62-70. Review. PubMed PMID: 28207917.

https://www.aacp.com/article/buy now/?id=464 


\section{Abstract}

Background: Posttraumatic stress disorder (PTSD) is associated with a high burden of disability and mortality and frequently is treatment-resistant. There is little to offer patients who are not responding to standard interventions. Thus, the objective of this report is to systematically review human data on whether electroconvulsive therapy (ECT) is effective in PTSD.

Methods: We performed a systematic literature review through August 2016 for clinical studies and case reports published in English examining the efficacy of ECT in improving PTSD symptoms.

Results: The literature search generated 3 retrospective studies, 1 prospective uncontrolled clinical trial, and 5 case reports. It is not clear given the small sample size and lack of a large randomized trial whether favorable outcomes were attributed to improvement in depression (as opposed to core PTSD symptoms).

Conclusions: Current efficacy data do not separate conclusively the effects of ECT on PTSD symptoms from those on depression. Randomized controlled trials are therefore necessary to examine the use of ECT in medication-refractory PTSD patients with and without comorbid depression. Subsequent studies may address response in PTSD subtypes, and the use of novel techniques such as memory reactivation before ECT. 


\section{INTRODUCTION}

The lifetime prevalence of posttraumatic stress disorder (PTSD) in the United States is approximately 7.8\% (R. C. Kessler, Chiu, Demler, Merikangas, \& Walters, 2005). The lifetime prevalence in other countries (using different survey administration and sampling strategies) for PTSD ranges from $0.3 \%$ in China to $6.1 \%$ in New Zealand (R. Kessler \& Ustun, 2008). PTSD is associated commonly with a high burden of disability, high suicide risk (Davidson, Hughes, Blazer, \& George, 1991) and functional impairment (Amaya-Jackson et al., 1999; Thomas et al., 2010). If not treated adequately, the prognosis often is chronic (R. C. Kessler, Sonnega, Bromet, Hughes, \& Nelson, 1995).

PTSD is frequently associated with other psychiatric comorbidities, which further complicates treatment strategies and response to treatment. This includes $45 \%$ comorbidity with major depression and more for depressive symptoms that are subsyndromal (Shalev et al., 1998). Suicidality (Davidson et al., 1991; Guerra, Calhoun, \& Mid-Atlantic Mental Illness Research, 2011) and substance use disorders (R. C. Kessler et al., 2005; Nock, Hwang, Sampson, \& Kessler, 2010; Wilcox, Storr, \& Breslau, 2009) further complicate PTSD (Dedert et al., 2009).

Individuals with PTSD also have higher rates of other physical disorders (Davidson et al., 1991) compared to those without PTSD in a community sample. Thus, those with PTSD have higher rates of mental and physical health services compared to those without PTSD (Calhoun, Bosworth, Grambow, Dudley, \& Beckham, 2002).

The first-line pharmacological treatment for PTSD is selective serotonin reuptake inhibitors (SSRIs). Nonetheless, less than one-half of patients achieve full remission (Amiel, Mangurian, Ganguli, \& Newcomer, 2008; Berger et al., 2009). Even fewer achieve full remission for combat-related PTSD and chronic PTSD (Davidson et al., 2003; Hertzberg, Feldman, Beckham, Kudler, \& Davidson, 2000). In addition, the effect size of improvement from SSRIs (standardized mean difference $-0.23,95 \% \mathrm{Cl}-0.33$ to -0.12 ) and pharmacotherapy (including studies of monoamine oxidase inhibitor, other antidepressants, olanzapine, and topiramate) in general has been found to be small according to a well-done meta-analysis (Hoskins et al., 2015).

Currently, sertraline (Brady et al., 2000; Schwartz \& Rothbaum, 2002) and paroxetine (Marshall et al., 2007; Tucker et al., 2001) are FDA-approved for PTSD. Nonetheless, many patients are unresponsive to first-line therapy with SSRI (Youssef et al., 2012). Also, SSRI trials have found that more than $50 \%$ of patients have incapacitating residual symptoms. A welldesigned trial of prazosin in active duty soldiers with PTSD found significant improvement in PTSD symptoms, especially nightmares, and sleep quality using prazosin. Although patients responded to prazosin, PTSD symptoms did not remit (mean Clinical-Administrated PTSD Scale (CAPS) score dropped from 77 to 52), and not all patients responded (Raskind et al., 2013). The effects of prazosin also have been demonstrated in civilian patients with PTSD (Taylor et al., 2008).

Pharmacotherapy and trauma-oriented psychotherapy (Cusack et al., 2016) are the most common treatment modalities used in clinical practice. Although these two modalities are mechanistically different and involve a multidisciplinary team, only approximately $50 \%$ of patients have remission of symptoms with both modalities. The other approximately $50 \%$ of patients remain treatment-resistant (R. C. Kessler et al., 1995). 
Electroconvulsive therapy (ECT) is the most effective treatment for major depressive (MDD) and bipolar disorders. Furthermore, it is also the most effective treatment for depressed patients who are treatment-resistant to pharmacotherapy and psychotherapy (Lisanby, 2007). However, PTSD is not a recognized indication for ECT. In this manuscript, we systematically review the available evidence in order to evaluate the effectiveness of ECT in treating PTSD, especially severe or treatment-resistant forms.

\section{METHODS}

We reviewed the medical literature for papers published on ECT treatment of patients with PTSD. We included reports in English from 1958 to August 2016 using PubMed/Medline. We also searched the Web of Science database and the Clinicaltrials.gov registry. We used the search terms: electroconvulsive therapy and posttraumatic stress disorder; electroconvulsive therapy and PTSD; ECT and PTSD. In PubMed, we also added the terms "Stress Disorders, PostTraumatic"[Mesh] AND "Electroconvulsive Therapy"[Mesh]. We also searched the bibliographies of the found articles and contacted several experts in the field for any possible additional publications/studies. As expected, there was overlap between the above searches. All papers were found in both PubMed and Web of Science except for Ozdemir et al.,(Ozdemir, Garip, Oznur, Erdem, \& Akarsu, 2013) which was only found in the Web of Science. Only one clinical trial was found in Clinicaltrials.gov registry, by Margoob et al.(Margoob, Ali, \& Andrade, 2010). See Figure 1 for PRISMA diagram. 
FIGURE

PRISMA diagram for the systematic literature review of original reports on the use of electroconvulsive therapy in patients with posttraumatic stress disorder
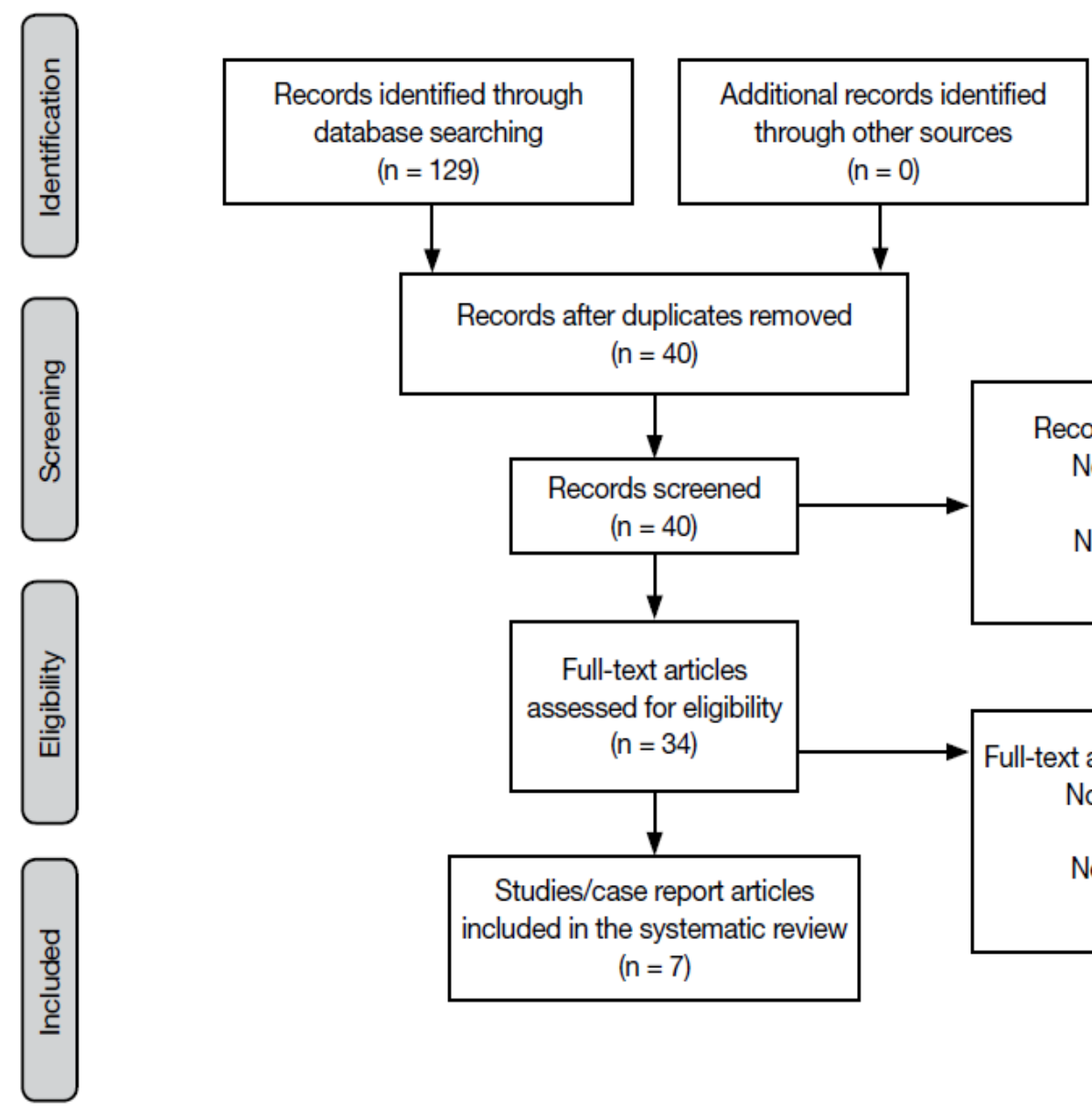

Records after duplicates removed $(n=40)$

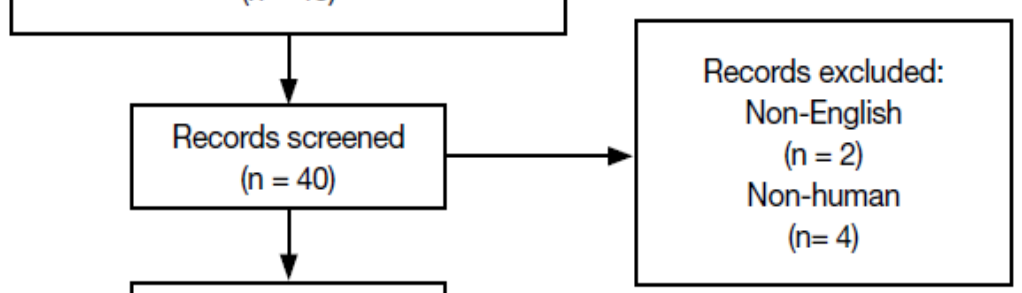

Full-text articles assessed for eligibility

$(n=34)$

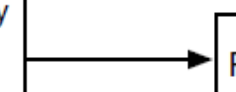

Full-text articles excluded:

Non-original $(n=10)$

Not in spirit

Studies/case report articles included in the systematic review $(\mathrm{n}=17)$

Source: Moher D, Liberati A, Tetzlaff J, et al; PRISMA Group. Preferred reporting items for systematic reviews and meta-analyses: the PRISMA statement. Ann Intern Med. 2009;151(4):264-269, W64

\section{RESULTS}

The literature search generated 7 published papers relevant to the efficacy of ECT in PTSD. There were no relevant papers published in languages other than English. Our findings included 3 studies (Table 1 ) and 5 case reports published in 4 papers (Table 2).

\section{Studies of PTSD}

In 2007, Watts (Watts, 2007) conducted a retrospective chart review study to examine the use of ECT for treatment of PTSD in 26 military veterans with PTSD and comorbid major depression. The study was carried out in a VA Hospital in the New England area (from January of 1998 to December of 2002). 
Out of 77 patients who were treated with an index course of ECT, 28 patients met the criterion of both current MDD and PTSD according to DSM-IV. Of those, 26 patients had complete baseline and endpoint assessments using PTSD Checklist (PCL)(Forbes, Creamer, \& Biddle, 2001) and the Montgomery-Åsberg Depression Rating Scale (MADRS)(Montgomery \& Asberg, 1979). These 26 patients were included in the statistical analysis. Sixteen patients started and completed the acute course of ECT while hospitalized, while 10 patients completed the course after hospital discharge.

Routine psychiatric medications were tapered and discontinued before ECT (as is usually the practice at that center). ECT was administered using MECTA SpECTrum 5000Q device (MECTA Corporation, Lake Oswego. OR). Anesthesia was administered with methohexital, and the muscle relaxant was succinylcholine. All patients started treatment with right unilateral ECT (administered at 2.5 times the seizure threshold). Twenty-one patients received unilateral treatments only (unilateral treatments sessions 9.7 [SD, 1.6]). Five patients who were nonresponders to right unilateral (RUL) ECT were switched after 8 treatments to bitemporal ECT (bitemporal treatments 4.6 [SD, 1.2]).

PTSD response was defined as $\geq 20 \%$ reduction in PTSD symptoms on the PCL. Depression response was defined as a $50 \%$ reduction in depressive symptoms, and remission was defined as $\geq 60 \%$ reduction plus final MADRS of $\leq 10$. The statistical analysis used a paired t-test comparing pre-treatment to post-treatment level of symptoms on the PCL and MADRS.

This study found that patients receiving ECT had a significant reduction in the symptoms of major depression and a statistically insignificant reduction in PTSD symptoms (Table 1). The authors concluded that "electroconvulsive therapy may be an effective treatment for patients with refractory depression and co-occurring PTSD." However, the major limitations were the small sample and the retrospective chart review nature of the study, which may have affected the accuracy of the PTSD diagnosis retrospectively.

A prospective open-label study by Margoob and Andrade (Margoob et al., 2010) enrolled 20 consecutive adult civilian patients with severe, chronic, treatment-resistant PTSD. Treatment resistance was defined as failure to respond to an adequate trial of at least 4 antidepressant medications and 12 sessions of cognitive-behavior therapy. Participants were treated with a fixed index course of 6 bilateral ECT sessions (sessions administered outpatient twice-weekly). Anesthesia was administered with pentothal; the muscle relaxant was succinylcholine. ECT was administered using the MECTA-SR1 device with current amplitude of $800 \mathrm{~mA}$, pulse width of 1.5 $\mathrm{ms}$, and frequency of $50 \mathrm{~Hz}$. Stimulus duration was increased as needed to elicit an adequate seizure. An adequate seizure was defined as an EEG seizure of least $25 \mathrm{~s}$. The stimulus intensity was barely suprathreshold. Psychotropic medications were tapered and stopped before the ECT course.

CAPS was used as the primary outcome measure. Response was defined as at least a $30 \%$ decrease in CAPS, and remission as a CAPS score of $<20$. Secondary outcome measures were the MADRS and the Clinical Global Impression-Severity scale (CGI-S).

Out of 20 participants, 17 completed the ECT course. An intent-to-treat analysis $(\mathrm{N}=20)$ showed a decrease in the CAPS scores by $34 \%(P<.001)$, and MADRS scores decreased by $51.1 \%$ 
$(P<.001)$. The authors reported that most of the improvement in PTSD and depression ratings developed by the third ECT session. It also was noted that the improvements in CAPS were independent of the improvements in MADRS. The authors noted that "improvement in CAPS did not differ significantly between patients with less severe vs more severe baseline depression". Statistical tests included paired $t$-test used to compare pre-post time points (before ECT, and after the sixth ECT session) and repeated measures ANOVA to compare across the three time points (before ECT, after the third, and the sixth ECT sessions).

A complete analysis also was performed for the 17 participants who completed the study. In those participants, CAPS scores dropped from 92 to 55 (40\%). The MADRS scores dropped from 24 to 10 (57\%). The CAPS response rate occurred in $82 \%$ of participants. The correlation between improvement in CAPS and improvement in MADRS was low and was not statistically significant.

Four of these patients had psychotic symptoms at baseline. In those patients, CAPS scores dropped from 86.5 to 47.0 (46\%). The MADRS scores dropped from 23.3 to 9.5 (59\%). The psychotic symptoms remitted in 3 patients (and were reduced markedly in the $4^{\text {th }}$ ). Treatment response (assessed clinically, not by rating scales) was maintained at a 4 to 6 month follow-up.

This was the only study in which PTSD was the indication for ECT (rather than depression or a psychotic disorder with comorbid PTSD in the other reports). On the other hand, patients were not formally assessed for comorbid MDD, and so the prevalence of MDD in the sample was not known.

In a retrospective, nested, matched case-control study, Ahmadi et al. (Ahmadi, Moss, Simon, Nemeroff, \& Atre-Vaidya, 2015) compared consecutive patients with PTSD and comorbid MDD who received ECT $(\mathrm{N}=92)$ to those with PTSD and comorbid MDD who did not receive ECT $(\mathrm{N}=3393)$.

ECT was performed using a Thymatron IV ECT device. All patients received bifrontal (BF) ECT, with stimulus dose administration at least $200 \%$ above the seizure threshold "based on agebased method". ECT was administered three times a week with an average of $6 \pm 1$ ECT sessions. Anesthesia consisted of pretreatment with glycopyrrolate, followed by induction with an anesthetic agent (methohexital, $0.75 \mathrm{mg} / \mathrm{kg}$; propofol $1 \mathrm{mg} / \mathrm{kg}$ in 5 cases) followed by succinylcholine $(0.75 \mathrm{mg} / \mathrm{kg})$ for muscle relaxation.

Improvement of PTSD and MDD symptoms, as assessed by clinical global impression scale (CGI) was better for ECT (90\%), compared to antidepressant medications (50\%) $(P=.001)$. Over a mean follow-up period of 8 years, they found that the ECT-treated group had statistically significant risk reductions of $64 \%$ for suicide, $65 \%$ for all-cause mortality, and $46 \%$ risk reduction for cardiovascular mortality relative to the group that did not receive ECT. Symptomatic improvement was also greater in the ECT-treated patients. 
TABLE 1

Electroconvulsive therapy studies in posttraumatic stress disorder

\begin{tabular}{|c|c|c|c|c|c|c|}
\hline $\begin{array}{l}\text { Author(s)/years } \\
\text { of the study } \\
\text { period }\end{array}$ & $\mathrm{N}$ & Design & Sample & EP and (XST) & PW & $\begin{array}{l}\text { Definition of response } \\
\text { and remission }\end{array}$ \\
\hline $\begin{array}{l}\text { Margoob et al } \\
(2010) \text { (January } \\
1,2005 \text {, to } \\
\text { December } 31 \text {, } \\
2005)^{28}\end{array}$ & 20 & $\begin{array}{l}\text { Open-label, } \\
\text { prospective } \\
\text { study }\end{array}$ & $\begin{array}{l}\text { All "consenting" adults with } \\
\text { PTSD (DSM-IV), outpatients } \\
\text { All eligible outpatients } \\
\text { undergoing ECT participated. } \\
\text { Age range: } 21 \text { to } 61 \text { years; mean } \\
38.0 \text { (SD 12.1); } 50 \% \text { males; } \\
\text { Duration of PTSD ranged from } \\
18 \text { to } 95 \text { months, mean } 35.9 \text { (SD } \\
\text { 18.7) months }\end{array}$ & $\begin{array}{l}\text { Fixed course } \\
\text { of } 6 \mathrm{BL} \text {, } \\
\text { twice-weekly } \\
\text { outpatient }\end{array}$ & $1.5 \mathrm{~ms}$ & $30 \%$ reduction in CAPS \\
\hline $\begin{array}{l}\text { Watts (2007) } \\
\text { (January } 1998 \\
\text { to December } \\
2002)^{29}\end{array}$ & 26 & $\begin{array}{l}\text { Retrospective } \\
\text { chart review }\end{array}$ & $\begin{array}{l}\text { Veterans with PTSD and } \\
\text { comorbid MDD (DSM-IV) }\end{array}$ & $\begin{array}{l}\text { All started } \\
\text { RUL (2.5); } 10 \\
\text { switched } \\
\text { to BL }\end{array}$ & - & $\begin{array}{l}\text { Depression, response } 50 \% \\
\text { reduction on MADRS, } \\
\text { remission } \geq 60 \% \text { reduction } \\
\text { and a final MADRS }+\leq 10 \\
\text { score. PTSD response } \geq 20 \% \\
\text { reduction on PCL }\end{array}$ \\
\hline $\begin{array}{l}\text { Ahmadi et al } \\
\text { (2016) } \\
\left(2004 \text { to 2013) }{ }^{32}\right.\end{array}$ & $\begin{array}{l}92 \text { received } \\
\text { ECT, } 3393 \\
\text { did not } \\
\text { receive ECT }\end{array}$ & $\begin{array}{l}\text { Retrospective, } \\
\text { nested, with } \\
\text { matched } \\
\text { case-control }\end{array}$ & $\begin{array}{l}\text { Patients with PTSD and } \\
\text { comorbid MDD who had ( } n=92) \\
\text { and had not }(n=3393) \text { received } \\
\text { ECT. Mean study period was } 8 \\
\text { years }\end{array}$ & $\begin{array}{l}\text { Bifrontal ECT } \\
\text { (at least 200\% } \\
\text { XST) }\end{array}$ & - & - \\
\hline
\end{tabular}

BL: bilateral ECT; CAPS: Clinician-Administrated PTSD scale, CGI-S: Clinical Global Impression-Severity; ECT: electroconvulsive therapy; EP: electrode placement; MADRS: Montgomery-Åsberg Depression Rating Scale; MDD: major depressive disorder; ms: milliseconds; PCL: PTSD Checklist; PTSD: posttraumatic stress disorder; PW: pulse width; RUL: right unilateral ECT; SD: standard deviation; XST: times above seizure threshold. 


\begin{tabular}{|c|c|}
\hline $\begin{array}{l}\text { Primary outcome } \\
\text { measure(s) }\end{array}$ & $\begin{array}{l}\text { Changes in primary outcome } \\
\text { measure(s) }\end{array}$ \\
\hline $\begin{array}{l}\text { CAPS } \\
\text { Secondary: MADRS, } \\
\text { CGI-S }\end{array}$ & $\begin{array}{l}\text { A fixed course of } 6 \text { bilateral ECT sessions } \\
\text { resulted in a CAPS improvement of } 34 \% \text {, } \\
\text { and MADRS improvement of } 51 \% \\
\text { Most improvement in both CAPS and } \\
\text { MADRS occurred by the third ECT session. } \\
\text { It was noted that the response rate was } \\
70 \% \text { in the intention-to-treat analysis and } \\
82 \% \text { in completers; no patients remitted. } \\
\text { Treatment gains were maintained at a } 4 \\
\text { to } 6 \text { month follow-up. However, this was } \\
\text { assessed clinically and not by rating scales }\end{array}$ \\
\hline PCL, MADRS & $\begin{array}{l}\text { Although the symptoms of MDD were } \\
\text { reduced significantly, symptoms of PTSD } \\
\text { showed some improvement. "The subjects } \\
\text { showed a reduction in PTSD, as measured } \\
\text { using the PCL, from } 71.08 \text { (SD, } 4.72 \text { ) before } \\
\text { ECT to } 55.62 \text { (SD, } 8.84 \text { ) after ECT ( } P>.001 \text { ). } \\
\text { Thirty-five percent of subjects showed a } \\
20 \% \text { reduction in symptoms, meeting the } \\
\text { established criterion for PTSD response" } \\
\text { The response rate for MDD was } 50 \% \text {, and } \\
\text { the remission rate was } 42 \%\end{array}$ \\
\hline $\begin{array}{l}\text { Symptomatic } \\
\text { improvement using } \\
\text { CGI-S and change } \\
\text { scales at baseline and } 3 \\
\text { month follow up } \\
\text { The long-term end points } \\
\text { were occurrence of } \\
\text { cardiovascular, all-cause } \\
\text { mortality, and suicide }\end{array}$ & $\begin{array}{l}\text { Symptomatic improvement was greater } \\
\text { in the ECT-treated patients. Moreover, } \\
\text { ECT-treated group had a risk reduction } \\
\text { of } 64 \% \text { for suicide, } 65 \% \text { reduction for } \\
\text { all-cause mortality, and } 46 \% \text { reduction for } \\
\text { cardiovascular mortality compared with } \\
\text { those who did not received ECT }\end{array}$ \\
\hline
\end{tabular}

\section{Case reports}

Our search also produced 5 case reports on the use of ECT in individuals with PTSD. The first case describes a 35-year-old woman who had severe combat-related PTSD and depression. The PTSD symptoms were resistant to standard therapies of both medication and psychotherapy (Helsley, Sheikh, Kim, \& Park, 1999). The patient underwent an acute course of 6 unilateral ECT sessions (3 times per week) after discontinuation of all psychotropic medications. PTSD symptoms responded "substantially" to the acute ECT course.

Another case depicts a 38-year-old woman who had medication-refractory depression and PTSD. The patient also had complete remission of depression and PTSD symptoms after 8 bilateral ECT sessions (Hanretta \& Malek-Ahmadi, 2006). In both of the above cases, no rating 
scales were used, but rather diagnoses and symptoms remission were based on clinical assessment (Table 2).

A third recently published case report utilized conversation-based reactivation of traumatic memory contents in PTSD and subsequent ECT-mediated impairment of reconsolidation (Gahr, Schonfeldt-Lecuona, Spitzer, \& Graf, 2014). The patient also was followed for 12 weeks after the last ECT session. The patient was assessed with rating scales for PTSD (Clinician-Administered PTSD scale, Part 2 [CAPS-2])(Blake et al., 1995) and depression (Beck Depression Inventory II [BDI])(Beck, Steer, \& Brown, 1996), MADRS, and Hamilton Rating Scale for Depression (Hamilton, 1960).

It might be argued that the efficacy of ECT in PTSD is due to impaired memory and thus impaired reactivation of traumatic memories. Memory contents are thought to be destabilized during retrieval of such memories, lending them sensitive to modifications (Schwabe, Nader, Wolf, Beaudry, \& Pruessner, 2012). This was the premise for this case, assuming that ECT might be "most effective immediately after an active (conversation-based) confrontation" with traumatic memories.

The patient received 8 sessions of right unilateral ECT ( 3 sessions per week). The ECT parameters and device were not specified. The authors also reported that they "did not observe any significant side effects related to ECT". Sertraline, $200 \mathrm{mg} / \mathrm{d}$, and trimipramine, $75 \mathrm{mg} / \mathrm{d}$ were co-administered during the ECT procedure (and after the ECT course). The patient had PTSD that was treatment resistant for 7 years to both psychotherapy and pharmacotherapy. After ECT, the patient experienced improvement in PTSD symptoms. Depressive symptoms also improved. The authors explain the improvement is due to possibly "successful ECT-mediated extinction" of traumatic memory.

The fourth and fifth cases were published by Ozdemir et al. (Ozdemir et al., 2013). The fourth case presents a 40-year-old male who developed PTSD and psychotic disorder not otherwise specified (NOS) after combat per DSM-IV-TR. He suffered a range of symptoms including recurring nightmares, hyper-arousal, and insomnia as well as auditory and visual hallucinations, persecutory delusions, and disorganized and violent behaviors. After hospitalization, the patient was started on risperidone $4 \mathrm{mg} / \mathrm{d}$ and venlafaxine $225 \mathrm{mg} / \mathrm{d}$. There was no improvement after 6 weeks of pharmacotherapy, thus he was offered a course of 10 sessions of bilateral ECT, 3 sessions per week, along with the same doses of the medications, using the Thymatron device (System IV; Somatics, Lake Bluff, III), with a stimulus titration schedule beginning at 25\% (charge, $126 \mathrm{mC}$; frequency, $30 \mathrm{~Hz}$; duration, $2.33 \mathrm{~s}$; current, $0.9 \mathrm{~A}$; and pulse width, $1.0 \mathrm{~ms}$ ) until seizure threshold of $60 \%$ was attained. Each stimulus resulted in seizure duration ranging from 20 to 45s. (It was not specified if subsequent treatments were subsequent treatments at threshold, or at a higher level.) Psychotic and PTSD symptoms improved, and the authors reported that after the $10^{\text {th }}$ session of ECT, both PTSD and psychotic symptoms resolved. Brief Psychiatric Rating Scale (BPRS) score improved by $33.6 \%$ (baseline 44 ), and the CAPS improved by $37.3 \%$ (baseline 67).

The fifth case also presents a 42-year-old man diagnosed with PTSD and psychotic disorder NOS after combat-related trauma, according to DSM-IV criteria. The patient presented with re-experiencing traumatic events, auditory and visual hallucinations, persecutory delusions, 
social isolation, and hearing sounds of dead soldiers. He was admitted for hospitalization and also was started on risperidone, $4 \mathrm{mg} / \mathrm{d}$, and venlafaxine, $225 \mathrm{mg} / \mathrm{d}$. There was "insignificant improvement" with medications for 6 weeks. Subsequently, the patient underwent ECT (8 sessions, electrode placement not mentioned) along with the medication. Both PTSD and psychotic symptoms improved after the 8 ECT sessions. Baseline BPRS score of 42 improved by $35.2 \%$, and baseline CAPS score of 66 improved by $37.2 \%$. Two weeks after the ECT course, the dose of risperidone was reduced to $1 \mathrm{mg} / \mathrm{d}$. The symptoms continued to improve with a decrease in BPRS by $65.1 \%$ and a decrease in CAPS by $44.5 \%$ from baseline.

TABLE 2

Electroconvulsive therapy case reports in posttraumatic stress disorder

\begin{tabular}{|c|c|c|c|c|c|}
\hline Study & Age, sex, $\mathrm{N}$ & Diagnosis & ECT course & $\begin{array}{l}\text { ECT device/ } \\
\text { parameters }\end{array}$ & $\begin{array}{l}\text { Psychotropics during } \\
\text { the ECT course }\end{array}$ \\
\hline \multirow[t]{2}{*}{$\begin{array}{l}\text { Özdemir et al } \\
(2013)^{27}\end{array}$} & $\begin{array}{l}\text { 40-year-old } \\
\text { male }\end{array}$ & $\begin{array}{l}\text { Combat-related PTSD } \\
\text { and comorbid psychosis } \\
\text { NOS }\end{array}$ & 10 BL ECT sessions & $\begin{array}{l}\text { Thymatron device } \\
\text { IV. Titration schedule } \\
\text { beginning at } 25 \% \\
\text { with charge, } 126 \mathrm{mC} \text {; } \\
\text { frequency, } 30 \mathrm{~Hz} \text {; } \\
\text { duration, } 2.33 \mathrm{~seconds} \text {; } \\
\text { current, } 0.9 \mathrm{~A} \text {; and } \\
\text { pulse width, } 1.0 \mathrm{~ms} \\
\text { was increased until } \\
\text { seizure threshold of } \\
60 \% \text { was attained. } \\
\text { Each stimulus resulted } \\
\text { in seizure duration from } \\
20 \text { to } 45 \mathrm{~s}\end{array}$ & $\begin{array}{l}\text { Risperidone, } 4 \mathrm{mg} / \mathrm{d} \text {, and } \\
\text { venlafaxine, } 225 \mathrm{mg} / \mathrm{d}\end{array}$ \\
\hline & $\begin{array}{l}\text { 42-year-old } \\
\text { male }\end{array}$ & $\begin{array}{l}\text { Combat-related PTSD } \\
\text { and comorbid psychosis } \\
\text { NOS }\end{array}$ & $\begin{array}{l}8 \text { ECT sessions; } \\
\text { electrode placement } \\
\text { not mentioned }\end{array}$ & Not mentioned & $\begin{array}{l}\text { Risperidone, } 4 \mathrm{mg} / \mathrm{d} \text {, and } \\
\text { venlafaxine, } 225 \mathrm{mg} / \mathrm{d}\end{array}$ \\
\hline $\begin{array}{l}\text { Helsley et al } \\
(1999)^{33}\end{array}$ & $\begin{array}{l}\text { 35-year-old } \\
\text { woman }\end{array}$ & $\begin{array}{l}\text { Severe combat-related } \\
\text { treatment-resistant } \\
\text { PTSD and depression }\end{array}$ & $\begin{array}{l}\text { Index course of } 6 \\
\text { unilateral ECT, } 3 \text { per } \\
\text { week }\end{array}$ & -- & $\begin{array}{l}\text { Discontinuation of all } \\
\text { psychotropic medications }\end{array}$ \\
\hline $\begin{array}{l}\text { Hanretta and } \\
\text { Malek-Ahmadi } \\
(2006)^{34}\end{array}$ & $\begin{array}{l}\text { 38-year-old } \\
\text { woman }\end{array}$ & $\begin{array}{l}\text { Medication-refractory } \\
\text { depression and PTSD }\end{array}$ & 8 BL ECT sessions & $\begin{array}{l}\text { Thymatron DG. Energy } \\
\text { dial was set at } 40 \% \\
\text { (frequency, } 50 \mathrm{~Hz} \text {; } \\
\text { duration, } 2.24 \mathrm{~seconds} \text {; } \\
\text { number of pulses, } 224 \text {; } \\
\text { charge, } 201.6 \mathrm{mC} \text { ) } \\
\text { Seizure duration range, } \\
26 \text { to } 44 \text { seconds }\end{array}$ & $\begin{array}{l}\text { Combination of } \\
\text { duloxetine, } 60 \mathrm{mg} / \mathrm{d} \text {, and } \\
\text { olanzapine, } 15 \mathrm{mg} / \mathrm{d}\end{array}$ \\
\hline $\begin{array}{l}\text { Gahr et al } \\
(2014)^{35}\end{array}$ & $\begin{array}{l}\text { 49-year-old } \\
\text { man }\end{array}$ & $\begin{array}{l}\text { Psychotherapy and } \\
\text { pharmacotherapy- } \\
\text { refractory PTSD for } 7 \\
\text { years (PTSD assessed } \\
\text { with CAPS-2) }\end{array}$ & $\begin{array}{l}\text { Conversation-based } \\
\text { reactivation of } \\
\text { traumatic memory } \\
\text { contents in PTSD, } \\
\text { followed by } 8 \text { RUL ECT } \\
\text { sessions ( } 3 \text { per week) }\end{array}$ & $\begin{array}{l}\text { ECT parameters } \\
\text { and device were not } \\
\text { specified }\end{array}$ & $\begin{array}{l}\text { Combination of } \\
\text { sertraline, } 200, \mathrm{mg} / \mathrm{d} \text {, and } \\
\text { trimipramine, } 75 \mathrm{mg} / \mathrm{d}\end{array}$ \\
\hline
\end{tabular}

BL: bilateral; BPRS: Brief Psychiatric Rating Scale; CAPS-2: Clinician-Administered PTSD scale, Part 2; ECT: electroconvulsive therapy; ms: milliseconds; NOS: not otherwise specified; PTSD: posttraumatic stress disorder; RUL: right unilateral. 


\section{DISCUSSION}

This review found limited publication on the treatment of PTSD, mainly treatmentresistant PTSD, using ECT. The available preliminary data suggest modest improvement in the severity of PTSD symptoms, especially in patients with concurrent PTSD and MDD. The improvement is not correlated merely with improvement in depressive symptoms (as might be thought intuitively).

A sizable portion of patients with PTSD is treatment-resistant (Brady et al., 2000; Davidson, Rothbaum, van der Kolk, Sikes, \& Farfel, 2001; Youssef et al., 2012). In non-treatmentresistant patients, response rates to approved pharmacotherapy are $50 \%$ to $60 \%$ at best (Brady et al., 2000; Davidson, Tharwani, \& Connor, 2002; Marshall, Beebe, Oldham, \& Zaninelli, 2001; Tucker et al., 2001). In the only clinical trial and the most rigorously available in the literature so far, by Margoob et al.(Margoob et al., 2010), the intention-to-treat response rate to ECT for PTSD in this pharmacotherapy- and psychotherapy-resistant sample was $70 \%$. If that holds in a larger controlled trial, it will offer substantial promise for treatment-resistant PTSD patients. Also, the participants had improvements after 6 ECT sessions. It is also possible that treatment response would be maintained or improved after a longer acute course analogous to the standard for MDD. Finally the techniques of memory reactivation before ECT procedure may be another important direction of research (Gahr et al., 2014).

There are several limitations regarding the conclusions that can be drawn from the available literature. First, this systematic review is limited by the small number of published reports and the small number of participants in most studies. It is also limited by the design of retrospective studies and one open-label prospective studies, and by the lack of randomized clinical trials. Second, it is hard to rule out the potential placebo response of ECT on PTSD symptoms in such studies with a small sample size and non-controlled studies, as well as the potential effect of hospitalization while receiving psychosocial treatment. Third, the possibility of publication bias (in which studies with negative outcomes are not published) could not be ruled out, especially in the absence of rigorously done randomized controlled trials in the literature.

As a result of these limitations, it is unclear whether ECT is effective for PTSD. As there is not enough evidence to support its efficacy so far, it is hard to ascertain if PTSD symptoms have improved only because of improvement in depression or if there is a true anti-PTSD effect. However, there is no evidence of it causing harm either. It is possible that a subset of patients with PTSD will respond to ECT. Moreover, accumulating evidence supports the notion that continuing antidepressants during ECT improves MDD outcomes (Youssef \& McCall, 2014). Similarly, it is probable that discontinuation of antidepressants in the 2 studies of the use of ECT in PTSD available in the literature (as well as in a case report) might have interfered with ECT outcomes for these patients. More rigorous and well-powered controlled clinical trials are needed, however, for more conclusive and more definitive results. 
Disclosure: Dr. Youssef has received research support from the U.S. Department of Veterans Affairs and the Augusta Biomedical Research Corporation, and is a speaker for CME honoraria from the Georgia Department of Behavioral Health and Developmental Disabilities. Dr. McCall has received research support from American Foundation for Suicide Prevention, MECTA Corporation, Merck Pharmaceuticals, and the National Institute of Mental Health; is a speaker for CME Outfitters; is a consultant to Anthem Insurance, Merck, and Multiple Energy Technologies; and has received royalties from Wolters Kluwer. Dr. Andrade reports no financial relationships with any company whose products are mentioned in this article or with manufacturers of competing products. 
Contributions (added for the purpose of this thesis): Nagy A. Youssef, W. Vaughn McCall, and Chittaranjan Andrade conceptualized the idea of this systematic review and developed the inclusion criteria. Nagy Youssef developed the search strategy, searched the literature, and wrote the initial draft of the manuscript. All coauthors contributed intellectually and edited the later versions of the paper. All coauthors approved the final version of the manuscript. 


\section{REFERENCES}

Ahmadi, N., Moss, L., Simon, E., Nemeroff, C. B., \& Atre-Vaidya, N. (2015). Efficacy and longterm clinical outcome of comorbid posttraumatic stress disorder and major depressive disorder after electroconvulsive therapy. Depress Anxiety, 33(7), 640-647. doi:10.1002/da.22451

Amaya-Jackson, L., Davidson, J. R., Hughes, D. C., Swartz, M., Reynolds, V., George, L. K., \& Blazer, D. G. (1999). Functional impairment and utilization of services associated with posttraumatic stress in the community. J Trauma Stress, 12(4), 709-724. doi:10.1023/A:1024781504756

Amiel, J. M., Mangurian, C. V., Ganguli, R., \& Newcomer, J. W. (2008). Addressing cardiometabolic risk during treatment with antipsychotic medications. Curr Opin Psychiatry, 21(6), 613-618. doi:10.1097/YCO.0b013e328314b74b

Beck, A., Steer, R., \& Brown, G. (1996). BDI-II Manual. San Antonio, TX: The Psychological Corporation.

Berger, W., Mendlowicz, M. V., Marques-Portella, C., Kinrys, G., Fontenelle, L. F., Marmar, C. R., \& Figueira, I. (2009). Pharmacologic alternatives to antidepressants in posttraumatic stress disorder: a systematic review. Prog Neuropsychopharmacol Biol Psychiatry, 33(2), 169-180. doi:10.1016/j.pnpbp.2008.12.004

Blake, D. D., Weathers, F. W., Nagy, L. M., Kaloupek, D. G., Gusman, F. D., Charney, D. S., \& Keane, T. M. (1995). The development of a Clinician-Administered PTSD Scale. J Trauma Stress, 8(1), 75-90. doi:10.1002/jts.2490080106

Brady, K., Pearlstein, T., Asnis, G. M., Baker, D., Rothbaum, B., Sikes, C. R., \& Farfel, G. M. (2000). Efficacy and safety of sertraline treatment of posttraumatic stress disorder: a randomized controlled trial. Jama, 283(14), 1837-1844. doi:10.1001/jama.283.14.1837

Calhoun, P. S., Bosworth, H. B., Grambow, S. C., Dudley, T. K., \& Beckham, J. C. (2002). Medical service utilization by veterans seeking help for posttraumatic stress disorder. Am J Psychiatry, 159(12), 2081-2086. doi:10.1176/appi.ajp.159.12.2081

Cusack, K., Jonas, D. E., Forneris, C. A., Wines, C., Sonis, J., Middleton, J. C., . . . Gaynes, B. N. (2016). Psychological treatments for adults with posttraumatic stress disorder: a systematic review and meta-analysis. Clin Psychol Rev, 43, 128-141. doi:10.1016/j.cpr.2015.10.003

Davidson, J. R., Hughes, D., Blazer, D. G., \& George, L. K. (1991). Post-traumatic stress disorder in the community: an epidemiological study. Psychol Med, 21(3), 713-721. doi:10.1017/S0033291700022352

Davidson, J. R., Rothbaum, B. O., van der Kolk, B. A., Sikes, C. R., \& Farfel, G. M. (2001). Multicenter, double-blind comparison of sertraline and placebo in the treatment of posttraumatic stress disorder. Arch Gen Psychiatry, 58(5), 485-492.

doi:10.1001/archpsyc.58.5.485 
Davidson, J. R., Tharwani, H. M., \& Connor, K. M. (2002). Davidson Trauma Scale (DTS): normative scores in the general population and effect sizes in placebo-controlled SSRI trials. Depress Anxiety, 15(2), 75-78. doi:10.1002/da.10021

Davidson, J. R., Weisler, R. H., Butterfield, M. I., Casat, C. D., Connor, K. M., Barnett, S., \& van Meter, S. (2003). Mirtazapine vs. placebo in posttraumatic stress disorder: a pilot trial. Biol Psychiatry, 53(2), 188-191. doi:10.1016/S0006-3223(02)01411-7

Dedert, E. A., Green, K. T., Calhoun, P. S., Yoash-Gantz, R., Taber, K. H., Mumford, M. M., . . . Beckham, J. C. (2009). Association of trauma exposure with psychiatric morbidity in military veterans who have served since September 11, 2001. J Psychiatr Res, 43(9), 830836. doi:10.1016/j.jpsychires.2009.01.004

Forbes, D., Creamer, M., \& Biddle, D. (2001). The validity of the PTSD checklist as a measure of symptomatic change in combat-related PTSD. Behav Res Ther, 39(8), 977-986. doi:10.1016/S0005-7967(00)00084-X

Gahr, M., Schonfeldt-Lecuona, C., Spitzer, M., \& Graf, H. (2014). Electroconvulsive therapy and posttraumatic stress disorder: first experience with conversation-based reactivation of traumatic memory contents and subsequent ECT-mediated impairment of reconsolidation. J Neuropsychiatry Clin Neurosci, 26(3), E38-39. doi:10.1176/appi.neuropsych.13070159

Guerra, V. S., Calhoun, P. S., \& Mid-Atlantic Mental Illness Research, Education Clinical Center Workgroup. (2011). Examining the relation between posttraumatic stress disorder and suicidal ideation in an OEF/OIF veteran sample. J Anxiety Disord, 25(1), 12-18. doi:10.1016/j.janxdis.2010.06.025

Hamilton, M. (1960). A rating scale for depression. J Neurol Neurosurg Psychiatry, 23, 56-62. doi:10.1136/jnnp.23.1.56

Hanretta, A. T., \& Malek-Ahmadi, P. (2006). Combined use of ECT with duloxetine and olanzapine: a case report. J ect, 22(2), 139-141. Retrieved from http://www.ncbi.nlm.nih.gov/pubmed/16801831

Helsley, S., Sheikh, T., Kim, K. Y., \& Park, S. K. (1999). ECT therapy in PTSD. Am J Psychiatry, 156(3), 494-495. Retrieved from http://www.ncbi.nlm.nih.gov/pubmed/10080573

Hertzberg, M. A., Feldman, M. E., Beckham, J. C., Kudler, H. S., \& Davidson, J. R. (2000). Lack of efficacy for fluoxetine in PTSD: a placebo controlled trial in combat veterans. Ann Clin Psychiatry, 12(2), 101-105. doi:10.1023/A:1009076231175

Hoskins, M., Pearce, J., Bethell, A., Dankova, L., Barbui, C., Tol, W. A., . . Bisson, J. I. (2015). Pharmacotherapy for post-traumatic stress disorder: systematic review and metaanalysis. Br J Psychiatry, 206(2), 93-100. doi:10.1192/bjp.bp.114.148551

Kessler, R., \& Ustun, T. B. (Eds.). (2008). The WHO world mental health surveys: global perspectives on the epidemiology of mental disorders New York: Cambridge University Press. 
Kessler, R. C., Chiu, W. T., Demler, O., Merikangas, K. R., \& Walters, E. E. (2005). Prevalence, severity, and comorbidity of 12-month DSM-IV disorders in the National Comorbidity Survey Replication. Arch Gen Psychiatry, 62(6), 617-627. doi:10.1001/archpsyc.62.6.617

Kessler, R. C., Sonnega, A., Bromet, E., Hughes, M., \& Nelson, C. B. (1995). Posttraumatic stress disorder in the National Comorbidity Survey. Arch Gen Psychiatry, 52(12), 1048-1060. doi:10.1001/archpsyc.1995.03950240066012

Lisanby, S. H. (2007). Electroconvulsive therapy for depression. N Engl J Med, 357(19), 19391945. doi:10.1056/NEJMct075234

Margoob, M. A., Ali, Z., \& Andrade, C. (2010). Efficacy of ECT in chronic, severe, antidepressantand CBT-refractory PTSD: an open, prospective study. Brain Stimul, 3(1), 28-35. doi:10.1016/j.brs.2009.04.005

Marshall, R. D., Beebe, K. L., Oldham, M., \& Zaninelli, R. (2001). Efficacy and safety of paroxetine treatment for chronic PTSD: a fixed-dose, placebo-controlled study. Am J Psychiatry, 158(12), 1982-1988. doi:10.1176/appi.ajp.158.12.1982

Marshall, R. D., Lewis-Fernandez, R., Blanco, C., Simpson, H. B., Lin, S. H., Vermes, D., . . . Liebowitz, M. R. (2007). A controlled trial of paroxetine for chronic PTSD, dissociation, and interpersonal problems in mostly minority adults. Depress Anxiety, 24(2), 77-84. doi:10.1002/da.20176

Montgomery, S. A., \& Asberg, M. (1979). A new depression scale designed to be sensitive to change. Br J Psychiatry, 134, 382-389. doi:10.1192/bjp.134.4.382

Nock, M. K., Hwang, I., Sampson, N. A., \& Kessler, R. C. (2010). Mental disorders, comorbidity and suicidal behavior: results from the National Comorbidity Survey Replication. Mol Psychiatry, 15(8), 868-876. doi:10.1038/mp.2009.29

Ozdemir, B., Garip, B., Oznur, T., Erdem, M., \& Akarsu, S. (2013). Post-traumatic stress disorder and comorbid psychotic disorder: electroconvulsive therapy response in two patients. Klinik Psikofarmakoloji Bülteni, 23(4), 373-377. doi:10.5455/bcp.20130216021755

Raskind, M. A., Peterson, K., Williams, T., Hoff, D. J., Hart, K., Holmes, H., . . Peskind, E. R. (2013). A trial of prazosin for combat trauma PTSD with nightmares in active-duty soldiers returned from Iraq and Afghanistan. Am J Psychiatry, 170(9), 1003-1010. doi:10.1176/appi.ajp.2013.12081133

Schwabe, L., Nader, K., Wolf, O. T., Beaudry, T., \& Pruessner, J. C. (2012). Neural signature of reconsolidation impairments by propranolol in humans. Biol Psychiatry, 71(4), 380-386. doi:10.1016/j.biopsych.2011.10.028

Schwartz, A. C., \& Rothbaum, B. O. (2002). Review of sertraline in post-traumatic stress disorder. Expert Opin Pharmacother, 3(10), 1489-1499. doi:10.1517/14656566.3.10.1489

Shalev, A. Y., Freedman, S., Peri, T., Brandes, D., Sahar, T., Orr, S. P., \& Pitman, R. K. (1998). Prospective study of posttraumatic stress disorder and depression following trauma. Am J Psychiatry, 155(5), 630-637. doi:10.1001/jamaoto.2015.0540 
Taylor, F. B., Martin, P., Thompson, C., Williams, J., Mellman, T. A., Gross, C., . . Raskind, M. A. (2008). Prazosin effects on objective sleep measures and clinical symptoms in civilian trauma posttraumatic stress disorder: a placebo-controlled study. Biol Psychiatry, 63(6), 629-632. doi:10.1016/j.biopsych.2007.07.001

Thomas, J. L., Wilk, J. E., Riviere, L. A., McGurk, D., Castro, C. A., \& Hoge, C. W. (2010). Prevalence of mental health problems and functional impairment among active component and National Guard soldiers 3 and 12 months following combat in Iraq. Arch Gen Psychiatry, 67(6), 614-623. doi:10.1001/archgenpsychiatry.2010.54

Tucker, P., Zaninelli, R., Yehuda, R., Ruggiero, L., Dillingham, K., \& Pitts, C. D. (2001). Paroxetine in the treatment of chronic posttraumatic stress disorder: results of a placebocontrolled, flexible-dosage trial. J Clin Psychiatry, 62(11), 860-868. Retrieved from http://www.ncbi.nlm.nih.gov/entrez/query.fcgi?cmd=Retrieve\&db=PubMed\&dopt=Cita tion\&list uids $=11775045$

Watts, B. V. (2007). Electroconvulsive therapy for comorbid major depressive disorder and posttraumatic stress disorder. J ect, 23(2), 93-95. doi:10.1097/01.yct.0000264369.38033.34

Wilcox, H. C., Storr, C. L., \& Breslau, N. (2009). Posttraumatic stress disorder and suicide attempts in a community sample of urban American young adults. Arch Gen Psychiatry, 66(3), 305-311. doi:10.1001/archgenpsychiatry.2008.557

Youssef, N. A., Marx, C. E., Bradford, D. W., Zinn, S., Hertzberg, M. A., Kilts, J. D., . . Strauss, J. L. (2012). An open-label pilot study of aripiprazole for male and female veterans with chronic post-traumatic stress disorder who respond suboptimally to antidepressants. Int Clin Psychopharmacol, 27(4), 191-196. doi:10.1097/YIC.0b013e328352ef4e

Youssef, N. A., \& McCall, W. V. (2014). Relapse prevention after index electroconvulsive therapy in treatment-resistant depression. Ann Clin Psychiatry, 26(4), 288-296. Retrieved from http://www.ncbi.nlm.nih.gov/pubmed/25401716 



\section{Chapter 12 Feasibility, Safety, and Preliminary Efficacy of Low}

Amplitude Seizure Therapy (LAP-ST): A Proof of Concept Clinical Trial in Man

Nagy A. Youssef, ${ }^{1 *}$ Emad Sidhom ${ }^{2,3}$

${ }^{1}$ Department of Psychiatry and Health Behavior, Medical College of Georgia at Augusta University.

2 The Behman Hospital, Helwan, Cairo, Egypt.

${ }^{3}$ Oxford Health NHS Foundation Trust, UK

Published as: Youssef NA, Sidhom E. Feasibility, safety, and preliminary efficacy of Low Amplitude Seizure Therapy (LAP-ST): A proof of concept clinical trial in man. J Affect Disord. 2017 Nov;222:1-6. doi: 10.1016/j.jad.2017.06.022. Epub 2017 Jun 16. PubMed PMID: 28667887. https://www.sciencedirect.com/science/article/pii/S016503271631789X?via\%3Dihub 


\section{Abstract}

Background: Current pulse amplitude used in clinical ECT may be higher than needed. Reducing pulse amplitude may improve focality of the electric field and thus cognitive adverse effects. Here we examine the feasibility, safety, and whether Low Pulse Amplitude Seizure Therapy (LAP-ST, 0.5-0.6A) minimizes cognitive adverse effects while retaining efficacy.

Methods: Patients with treatment-resistant primary mood (depressive episodes) or psychotic disorders who were clinically indicated to undergo ECT were offered to be enrolled in an openlabel study. The study consisted of a full acute course of LAP-ST under standard anesthesia and muscle relaxation. The primary outcome was feasibility of seizure induction. Clinical outcome measures were: time to reorientation (TRO), Mini Mental State Examination, Montgomery Aberg Depression Scale, and Brief Psychiatric Rating Scale, and Clinical Global Impression Scale.

Results: Twenty-two patients consented to enrollment in the study. LAP-ST was feasible, and all patients had seizures in the first session. Participants had a quick orientation with median TRO of 4.5 minutes. Treatment was efficacious for both depressive and psychotic symptoms.

Limitations: Relatively small sample size, non-blinded, and no randomization was performed in this initial proof of concept study.

Conclusions: This first human preliminary data of a full course of focal LAP-ST demonstrates that seizure induction is feasible. These results, although preliminary, suggest that the LAP-ST compared to the standard ECT techniques may result in less cognitive side effects, but comparable efficacy. Larger studies are needed to replicate these findings.

\section{Keywords}

Electroconvulsive therapy; seizure therapy; low amplitude seizure therapy; ECT; seizure threshold, current titration; focal ECT; treatment-resistant depression; mood disorders; psychotic disorders.

TRIAL REGISTRATION: ClinicalTrials.gov NCT02928159 


\section{INTRODUCTION}

Mood and psychotic disorders have a huge negative impact on morbidity and mortality from mental and physical illness, with a substantial burden on the health of individuals, families, and the society (Murray \& Lopez, 1997; Szkultecka-Debek et al., 2016). Depression is predicted to be the second highest cause of disability after ischemic heart diseases by year 2020 (Murray \& Lopez, 1997). Treatment-resistant depression (TRD) was defined by the World Psychiatric Organization and the Massachusetts General Hospital Depression Program as failure to respond to an adequate trial of medication (Ayd, 1983; Fava \& Davidson, 1996). However, about a third of patients do not achieve remission despite 3 lines of adequate treatment trials as shown by the STAR*D clinical trial (Rush et al., 2006; Subedi, Aich, \& Sharma, 2016; Szkultecka-Debek et al., 2016). ECT is used for treatment-resistant schizophrenia usually in combination with antipsychotics (Tharyan \& Adams, 2005).

Electroconvulsive therapy (ECT) has been the most potent (UK ECT Review Group, 2003) and most rapid acting (Husain et al., 2004) form of intervention especially for the treatmentresistant mood and psychotic disorders in combination with medications (Griffiths, Zarate, \& Rasimas, 2014; Kellner, Husain, Knapp, McCall, Petrides, Rudorfer, Young, Sampson, McClintock, Mueller, Prudic, Greenberg, Weiner, Bailine, Rosenquist, Raza, Kaliora, Latoussakis, Tobias, Briggs, Liebman, Geduldig, Teklehaimanot, Lisanby, et al., 2016; Levy-Rueff, Jurgens, Loo, Olie, \& Amado, 2008; Medda et al., 2015; Minnai, Salis, Manchia, Pinna, \& Tondo, 2016; Palma et al., 2016; Prudic \& Sackeim, 1999; Subedi et al., 2016; Tharyan \& Adams, 2005; Youssef \& McCall, 2014). ECT can also help prevent relapse in treatment-resistant depression with continuation ECT and medications (Kellner, Husain, Knapp, McCall, Petrides, Rudorfer, Young, Sampson, McClintock, Mueller, Prudic, Greenberg, Weiner, Bailine, Rosenquist, Raza, Kaliora, Latoussakis, Tobias, Briggs, Liebman, Geduldig, Teklehaimanot, Dooley, et al., 2016; Youssef \& McCall, 2014).

Nonetheless, cognitive and memory-related adverse effects of ECT are still a major concern. Historically, modifications in ECT parameters in the form of ultra-brief pulse instead of brief pulse (Cronholm \& Ottosson, 1963a, 1963b) and the use of rectangular instead of sine wave (L. R. Squire \& Zouzounis, 1986; Weiner, 1980), as well as right unilateral (RUL) electrode placement instead of bilateral (BL) (L. R. Squire, 1977; S. R. Squire \& Slater, 1978) have lowered cognitive and memory adverse effects (Sackeim et al., 1986; Sackeim et al., 1993; Sackeim, Prudic, et al., 2000; Sackeim et al., 2008). However, despite these advances, memory and cognitive side effects are still experienced by a significant proportion of patients, constitute a barrier to treatment, and may, in part, contribute to the stigma associated with ECT (Matthews, Rosenquist, \& McCall, 2016; Sienaert, 2016). Thus, ECT is still underutilized as an effective life-saving intervention (Prudic \& Sackeim, 1999), despite the fact that ECT, when indicated, has a great impact on the quality of life and functionality of patients (McCall et al., 2013; McCall et al., 2011).

The current amplitude of the electric pulse is the primary factor that drives the electric field deeper in the brain structures (Peterchev, Krystal, Rosa, \& Lisanby, 2015; Peterchev, Rosa,

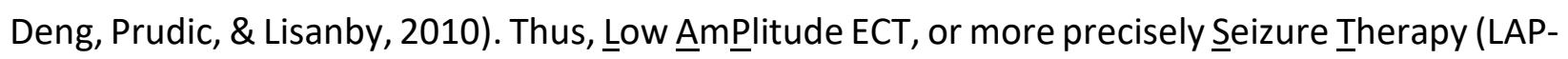


ST), should result in minimal spread of the electric field in the deeper hippocampal region and temporal lobe. (Peterchev et al., 2015; Peterchev et al., 2010). Avoidance or minimization of hippocampal and temporal lobe stimulation using LAP-ST is hypothesized to significantly reduce memory and cognitive adverse effects of ECT without affecting the more superficial stimulation of the Dorsolateral Prefrontal Cortex (DLPFC) and other cortical regions needed for the antidepressant effect (Noda et al., 2015).

Computational models of electric field induced in the brain using conventional high current amplitude is much higher than the neural activation threshold. This leads to deeper spread of the electric field in the brain. On the other hand, reducing pulse amplitude improves focality and decreases depth of penetration, thus potentially minimizing memory and cognitive adverse effects (Lee, Lisanby, Laine, \& Peterchev, 2012).

Low amplitude has been utilized in older ECT paradigms, (Friedman, 1942; Liberson, 1948) without anesthesia using older devices that have different specifications and parameters, such as being voltage constant (which leaves the current pulse amplitude to vary from patient to patient and session to session depending on the impedance). Modern ECT devices are currentconstant rather than voltage-constant. This allows for true fixed current amplitude. Thus, constantly minimizing the propagation of electric fields to the deeper memory areas of the brain that is associated with the cognitive adverse effects of standard ECT. Currently, in standard clinical practice, a high pulse amplitude of 800 to $900 \mathrm{~mA}$ is used (Lee, Lisanby, Laine, \& Peterchev, 2016). Also, in these older studies, anesthesia was not used, which would affect the seizure threshold (ST) estimates (Avramov, Husain, \& White, 1995).

In modern era, seizure titration during the first session using low amplitude was successfully reported in a letter by Rosa et al 2011 on 5 cases (Rosa, Abdo, Lisanby, \& Peterchev, 2011). These case reports involved one titration session, but no suprathreshold therapeutic dose sessions using low amplitude ECT.

Nahas et al performed a study to primarily examine investigational Focal Electrically Administered Seizure Therapy (FEAST) electrode placement and investigational unidirectional pulse (Nahas et al., 2013). In this study, low pulse amplitude were used to decrease an unusually high impedance at the smaller FEAST electrode. However, the primary aim of the study was to examine a novel electrode placement (along with a unidirectional current).

Although ECT has been in clinical practice for decades, so far, no human studies have primarily examined the feasibility or the effect of lower pulse amplitude of a full course on memory and cognition. Our hypothesis is that LAP-ST will reduce the cognitive burden of ECT. We also hypothesized that LAP-ST will be efficacious in both depressive and psychotic episodes. The aims of this study were to first examine the feasibility (primary outcome) of a full course of LAP-ST using low amplitude in humans and whether LAP-ST is associated with less cognitive adverse effects using Time to Orientation (as the main clinical outcome). We also explored the efficacy of LAP-ST. 


\section{METHODS}

\section{Participants.}

After IRB approval, patients were offered to be enrolled in the study if they were clinically indicated to undergo ECT, had capacity to consent for the study, and provided written informed consent. This study included patients with either primary mood or psychotic disorders who failed to respond to clinically adequate trials of psychopharmacological interventions in term of dosage and duration (with or without psychotherapy) as clinically indicated and were referred for ECT in Behman hospital in Cairo, Egypt. All patients were inpatients. Behman Hospital is a private psychiatric hospital accredited by the Royal College of Psychiatrists in England. Patients were enrolled between April 2013 to June 2015 after explanation of potential risks and any possible generalizable benefits of the study. The open-label study design involved a clinical course of LAPST typically given 3 times a week under standard anesthesia (sodium thiopental) and muscle relaxation (succinylcholine). There were no set minimum or maximum numbers of sessions.

\section{Eligibility.}

Inclusion criteria for enrollment were as follows: 1) ECT clinically indicated, 2) Males or females 22 to 80 years of age, 3) Use of effective method of birth control for women of childbearing capacity, 4) Patient is medically stable (i.e. no serious acute medical condition, generally assessed within the week before the ECT administration and on the morning of the ECT), 5) Capacity of patient to fully participate in the informed consent process as evaluated by their clinician.

Exclusion criteria included the following: 1) Current unstable or serious medical condition, or any co-morbid medical condition that substantially increases the risks of ECT, 2) History of conditions that may make ECT or anesthesia unsafe, 3) Female patients who are currently pregnant or plan to be pregnant during the study.

Patients were allowed to continue on their medications as clinically appropriate but on a fixed dose during the study.

\section{Assessments.}

Participants were diagnosed clinically according to Diagnostic and Statistical Manual of Mental Disorders, 4th Edition (DSM IV) criteria. Response and remission was determined clinically. Participants were also evaluated using Montgomery-Åsberg Depression Rating Scale (MADRS) (to measure change in depressive symptoms), Mini-Mental State Examination (for global cognitive function assessment) using standard Arabic translation (Al-Rajeh, Ogunniyi, Awada, Daif, \& Zaidan, 1999; Folstein, Folstein, \& McHugh, 1975) at baseline and endpoint. The 14-question Time to Reorientation test (TRO, the main cognitive measure) assessed the time to reorientation after ECT by asking 14 questions for person specific information (e.g., name, age), time information (e.g., date) and place information (e.g., location). The items are asked at 3, 5, 
10,15 , and 20 minutes following the end of seizure expression as documented on the EEG recording. The TRO score is the time it takes the patient to be able to answer 14/14 correctly. This test was modeled after and used by the PRIDE study workgroup (Kellner, Husain, Knapp, McCall, Petrides, Rudorfer, Young, Sampson, McClintock, Mueller, Prudic, Greenberg, Weiner, Bailine, Rosenquist, Raza, Kaliora, Latoussakis, Tobias, Briggs, Liebman, Geduldig, Teklehaimanot, Lisanby, et al., 2016). In addition to baseline and endpoint assessments, MADRS and MMSE were also done before each session, and TRO after each session. Patients diagnosed with schizophrenia or schizoaffective were also evaluated with Brief Psychiatric Rating Scale to measure change in psychotic symptoms (BPRS). Clinical Global Impression severity score (CGI-S) was used to assess overall change in clinical severity of symptoms. These scales were administered by an experienced staff psychiatrist (E.S). General side effects were assessed clinically by asking the patient if they have any side effects, or if patients simultaneously reported side effects that may be related to the ECT administration.

\section{Procedure.}

Patients received the standard clinical pre-ECT medical and laboratory workup as indicated by the treating clinicians for safe delivery of ECT and anesthetic procedures. Medication and anesthesia adjustments were used according to the discretion of the treating psychiatrist and ECT trained anesthesiologist as deemed clinically necessary according to the standard of care. A bite-block was inserted prior to seizure elicitation to protect the teeth. All other standard safety procedures for ECT were done as per the American Psychiatric Association ECT Guideline (American Psychiatric Association) (The Practice of Electroconvulsive Therapy: Recommendations for Treatment, Training, and Privileging: A Task Force Report of the American Psychiatric Association. (2001)). LAP-ST treatments during the acute course of this study were given three times per week (Kellner, Husain, Knapp, McCall, Petrides, Rudorfer, Young, Sampson, McClintock, Mueller, Prudic, Greenberg, Weiner, Bailine, Rosenquist, Raza, Kaliora, Latoussakis, Tobias, Briggs, Liebman, Geduldig, Teklehaimanot, Lisanby, et al., 2016).

\section{Low ÁmPlitude Seizure Therapy (LAP-ST).}

LAP-ST was performed using Mecta Spectrum 5000Q device and Right Unilateral (RUL) electrode placement. All seizure titrations were planned during the first session.

For seizure titration (ST) in the first session: Stimulus parameters were fixed at pulse width of $0.3 \mathrm{~ms}$ and duration of $8 \mathrm{~s}$. The seizure threshold was titrated using the current first from 500 to a maximum of $600 \mathrm{~mA}$ that was allowed in the study. Then, the frequency of the stimulus was titrated up if seizures were not induced; starting with frequency of $20 \mathrm{~Hz}$. Subsequent sessions change were 6 times that of the seizure threshold (that was determined in the first session). Tables 2 and 3 present further details of electric parameters, findings, duration of motor and EEG seizures, and anesthesia and muscle relaxation dosage.

\section{Anesthesia.}


Anesthesia was administered by an anesthesiologist with specific training and experience in ECT, as per standard anesthesia used for clinical ECT and the Hospital Policy and Procedure. Briefly, blood pressure, pulse, ECG, and pulse oximetry were monitored prior to anesthetic induction and continuously during the procedure. Standardized anesthesia procedures include induction with sodium thiopental $250 \mathrm{mg}$ and muscle relaxation with succinylcholine ( 0.75 $\mathrm{mg} / \mathrm{kg}$ ) or as determined clinically appropriate by the anesthesiologist. Patients were ventilated with $100 \%$ oxygen from anesthetic administration until the return of spontaneous respirations. Seizure expression was monitored by left fronto-mastoid EEG, and inspection of the cuffed foot to record motor manifestations. Both the duration of the EEG seizure and motor seizure duration were recorded in seconds.

\section{Electroencephalography (EEG) Acquisition and ictal monitoring.}

EEG seizure duration was monitored using two EEG channels (fronto-mastoid electrodes). The duration of the seizure in the brain was determined by the EEG recording (American Psychiatric Association) (The Practice of Electroconvulsive Therapy: Recommendations for Treatment, Training, and Privileging: A Task Force Report of the American Psychiatric Association. (2001)). Motor manifestations of seizures were monitored using the cuff technique. The cuff technique entails the placement of a blood pressure cuff on a limb. The cuff is then inflated above the systolic blood pressure immediately prior to the infusion of succinylcholine. Motor manifestations were observed and also monitored by Optical Motion Sensor (OMS) in combination with inspection of other cuffed foot and part of the body for motor seizures.

\section{Data analyses.}

The data was visualized and assessed for normality (i.e. normal distribution). Based on the normality of the data, or lack of normality, it was decided a priori to either use parametric or non-parametric tests, respectively. Due to the data not being normally distributed, it was reported using median and quartiles and compared by Wilcoxon Signed Ranks Test for paired data of the pre-post scores. TRO was reported with median and mean (to compare with historical controls). Wilcoxon Signed Ranks Tests were two tailed, and alpha was set a priori at $P=0.05$. SPSS version 23, (Inc., Chicago, IL, USA) was used for the statistical analyses. LOCF analysis was used in this pilot study.

\section{RESULTS}

\section{Demographic and clinical characteristics.}

Data from the total sample of 22 subjects are summarized in Table 1. Fifty percent (11) of participants from the total sample had a primary mood disorder diagnosis, and the other $50 \%$ had a primary psychotic disorder diagnosis as described in Table 1. All subjects, except for one, 
were taking psychotropic medication. The doses of the psychotropic medications were kept constant during the LAP-ST course.

\section{Treatment characteristics, parameters, and seizure duration.}

The dose of electrical stimulation and the electric parameters of the first titration session are described in Table 2, and those of subsequent suprathreshold sessions (sessions at 6 times seizure threshold) are described in Table 3. Patients were treated as determined clinically to respond, remit, decide to withdraw from the study, or were clinically determined as nonresponders by the ECT psychiatrist.

No patients had comorbid substance use disorder. This was determined by clinical history as well as negative drug screens. None of the patients were on prescribed benzodiazepines as per the hospital policy of not prescribing benzodiazepines during an ECT course.

Twenty-two patients underwent acute course of LAP-ST. All patients had seizure in the first session. No patients required more than 2 stimuli at the first session for seizure induction. The median number of sessions for the total sample was 6 .

\section{Feasibility and safety.}

The study showed feasibility of LAP-ST during a full course in humans. Threshold and suprathreshold seizures at 6 times seizure threshold were successfully induced in all patients.

One patient subjectively mentioned that for a few minutes after reorientation he had trouble focusing, however it was difficult to discern whether that was the effect of anesthesia and/or ECT. No participants subjectively complained of memory side effects during or after the LAP-ST course. Subjective memory problems were simply assessed by asking patients if they think they have any memory problems during or after ECT and as a result of it.

There were 3 patients who had headaches in the initial session which remitted after taking prophylactic ibuprofen the night before ECT sessions. Beyond that, there were no other reported or observed adverse effects related to the study.

\section{Cognitive assessment.}

The suprathreshold sessions for all participants during the LAP-ST course (excluding the first titration session) had a median time to full orientation (TRO) of $4.5 \mathrm{~min}(I Q R=3.2-10, N=20)$, and mean of 6.6 minutes. 
Median MMSE at baseline was 29.5 (IQR=27.8-30) and post LAP-ST was 30 (IQR=28-30), indicating no statistically significant difference $(p=0.6, z=0.6, N=18$ paired data, Wilcoxon Signed Ranks Test).

\section{Clinical efficacy.}

In patients who had a LAP-ST course of more than one suprathreshold treatment session (i.e. subsequent sessions at 6-times seizure threshold), the median Clinical Global ImpressionSeverity scales (CGI-S) improved from baseline of 6 down by 4 points (IQR $=2.5-4.5$ ) for patients in a depressive episode. For patients with schizophrenia, CGI-S improved from baseline of 5 down by 2.5 points (IQR = 1.25-3.0). CGI-S score for the total sample improved from baseline median of $5.5(I Q R=4-6)$ to endpoint of 3. However, this non-primary outcome (CGI-S) has some missing data and pre-post data for CGI were available for only 14 participants. The improvement in CGI$S$ was statistically significant $(p=0.002, z=-3.06)$.

On the MADRS, depression significantly improved ( $p 0.018, z=-2.4$, Wilcoxon Signed Ranks Test) in patients with unipolar or bipolar depression from baseline (median=34, IQR=12-46.5) to LAP-ST endpoint (median=10, IQR=6-36.5).

Psychotic symptoms (on BPRS) also significantly improved (Wilcoxon Signed Ranks Test $p=0.005, z=-2.8)$. The median improvement was $27(\mathrm{IQR}=21.5-32.3)$ in patients with schizophrenia or schizoaffective disorders from 73.5 (IQR=62-86.3) before LAP-ST to 47 (IQR=3652.8) after the LAP-ST.

\section{DISCUSSION}

This proof of concept study demonstrates that seizure induction using standard RUL electrode placement with focal LAP-ST course is both feasible and safe (with no significant side effects) in humans. This is considerable given the use of lower pulse amplitude along with thiopental, which probably leads to a higher seizure threshold compared to methohexital (Fond et al., 2016; Lihua, Su, Ke, \& Ziemann-Gimmel, 2014) (the most common anesthetic used in the US for ECT). In addition, as hypothesized LAP-ST seems to have reduced cognitive adverse effects (as measured by the TRO and MMSE). These data are promising and represent the first LAP-ST course in humans in modern times.

Similarly, in this preliminary study, efficacy was robust for both depressive and psychotic symptoms and seems comparable to studies of standard ECT. However, it should be noted that this proof of concept study warrants replication. 


\section{Acute cognitive side effects with LAP-ST versus standard ECT.}

Previous studies found that longer TRO is prospectively correlated with more long-term memory side effects with ECT (Martin, Galvez, \& Loo, 2015; Sackeim, Luber, et al., 2000; Sobin et al., 1995). TRO reported in published studies using standard ECT, with both RUL and bilateral ECT, were much longer (Kellner, Husain, Knapp, McCall, Petrides, Rudorfer, Young, Sampson, McClintock, Mueller, Prudic, Greenberg, Weiner, Bailine, Rosenquist, Raza, Kaliora, Latoussakis, Tobias, Briggs, Liebman, Geduldig, Teklehaimanot, Lisanby, et al., 2016; Kellner et al., 2010; Loo et al., 2014; Sackeim, Luber, et al., 2000; Sackeim et al., 2008) than the current study, predicting more memory adverse effects with standard ECT than with LAP-ST.

For instance, in a study by Sackeim and colleagues, 59 patients were randomized to either standard RUL or BL ECT administered at either "low dose" (just above ST) or "high dose" (150\% above ST). In this study, TRO was defined as the time after the end of seizure when patients could correctly answer 4 of 5 questions. Orientation questions were "presented continuously until positive response." The mean TRO ranged from $10.8 \mathrm{~min}(S D=8.3)$ for RUL low dose, $21.2 \mathrm{~min}$ $(S D=11.8)$ for RUL high dose, $39.4 \mathrm{~min}(S D=20.9)$ for $B L$ low dose and $42.7 \mathrm{~min}(S D=16.0)$ for $B L$ high dose ECT (Sackeim, Luber, et al., 2000). It should be noted that, generally speaking, low dose ECT means low total charge which is achieved by lowering the other electric parameter (such as the frequency or the duration of the stimulus) but not the current amplitude, while the current amplitude stays fixed at 800 or $900 \mathrm{~mA}$, depending on the device used in each study.

In another study by Kellner and colleagues (Kellner et al., 2010), reorientation score at 20 min after ECT was assessed using a ten-question instrument. Participants were randomized to receive bifrontal or BL ECT at 1.5 times above ST, or RUL ECT at 6 times above the ST. Averaged across all ECT sessions, except session one, it was reported that "the three electrode placements were not statistically different." The orientation time was assessed at $20 \mathrm{~min}$. The average questions answered correctly out of a total 10 questions were only 4.8 (SD $=2.5$ ) questions for bifrontal ECT, 5.7 (SD= 2.5) for RUL, and 5.5 (SD = 2.8) for BL (bitemporal). At 20 min, none of the electrode placement out of the 3 using high current amplitude was close to the full score of 10/10 correct questions.

Time to reorientation in other more recent published studies using ultra-brief pulse RUL ranged from 10-15 min, and for brief pulse RUL TRO ranged from 21-22 min (Kellner, Husain, Knapp, McCall, Petrides, Rudorfer, Young, Sampson, McClintock, Mueller, Prudic, Greenberg, Weiner, Bailine, Rosenquist, Raza, Kaliora, Latoussakis, Tobias, Briggs, Liebman, Geduldig, Teklehaimanot, Lisanby, et al., 2016; Loo et al., 2014; Sackeim et al., 2008). In a recent large clinical trial, the Prolonged Remission In Depressed Elderly (PRIDE), ultra-brief RUL ECT protocol was used for ECT in all participants reporting "relatively rapid reorientation, with close to half of patients being reoriented by 10 minutes." (PRIDE) (Kellner, Husain, Knapp, McCall, Petrides, Rudorfer, Young, Sampson, McClintock, Mueller, Prudic, Greenberg, Weiner, Bailine, Rosenquist,

Raza, Kaliora, Latoussakis, Tobias, Briggs, Liebman, Geduldig, Teklehaimanot, Lisanby, et al., 2016). Thus, it seems obvious that lower amplitude during a full course, as performed in this LAP- 
ST, provided even further benefit beyond standard ultra-brief RUL ECT that used high amplitude ECT.

Of note, the present study used more orientation questions (14 questions) and more rigorous cutoff to full orientation (14 out of 14 questions answered correctly) than many of the published studies, making a shorter TRO harder to achieve. Those who answered all 14 questions correctly did that in a mean TRO of $6.6 \mathrm{~min}$ and a median of 4.5 in the present study. Despite that, the present LAP-ST study had no participants who had mean TRO as long as $20 \mathrm{~min}$.

The mean MMSE scores at the end of the LAP-ST course showed no impairment, as an overall assessment of cognition throughout the LAP-ST course. In ECT using standard amplitude, MMSE scores typically show decline (Kellner et al., 2010; Sackeim, Luber, et al., 2000). For instance, in the one study, the mean MMSE dropped by 1.6 points from baseline to post ECT (Kellner et al., 2010). However, it should be noted that MMSE is not a sensitive measure of cognitive change and memory side effects in ECT literature, but that the decline in a short period (during one course) using standard ECT could also have some clinical importance. This could indicate even more significant clinical impairment in cognition over more frequent number of sessions or with multiple ECT courses (as in case of some treatment-resistant patients). Nonetheless, TRO is a more sensitive measure and predictive of memory side effects as explained earlier.

Weaknesses of the present study, however, include the use of only TRO and MMSE for assessment of cognition without additional cognitive tests. This was done due to the preliminary nature and limited budget of the study. As mentioned, however, TRO has been reliably shown in the ECT literature to correlate with memory problems prospectively (Lisanby, Luber, Schlaepfer, \& Sackeim, 2003; Sackeim, Luber, et al., 2000; Sackeim et al., 2008; Sobin et al., 1995). More extensive neurocognitive testing is warranted in larger studies. Secondly, this study had a relatively small sample size and was open-label and non-randomized. Further investigations using randomized clinical trial design with a larger sample size are warranted to support this first study in humans.

Nonetheless, data from this proof of concept study is very promising for the potential future utility of LAP-ST for treatment-resistant mood and psychotic disorders. A focal LAP-ST course seems to be efficacious with less memory side effects (as predicted by TRO) when compared to standard ECT. This line of research could substantially move the field forward in terms of reducing cognitive burden of ECT and offers groundwork for definitive studies for more focal seizure therapy. 
Acknowledgements: This study was presented at the Society of Biological Psychiatry $71^{\text {st }}$ Annual Meeting in Atlanta, Georgia (May 2016) and the International Society for ECT and Neurostimulation (ISEN) $26^{\text {th }}$ Annual Meeting in Atlanta, Georgia (May 2016).

This work was supported and sponsored by Behman Hospital (an accredited hospital by the Royal College of Psychiatrists, UK). We thank Drs. Nasser Loza and Sherif Attalla for their logistical support of this study. We acknowledge the work of Dr. Harold Sackeim in this area. We appreciate input from Drs. Sarah Lisanby, Angel Peterchev, Richard Weiner, and Andrew Krystal. We also thank Mark Yassa for his assistance with preparation of the tables and Susan Youssef for some edits in this manuscript.

ClinicalTrials.gov Identifier: NCT02928159

Funding: Dr. Nagy Youssef received research support from the Department of Veteran Affairs and The Augusta Biomedical Research Corporation for PTSD study. Dr. Youssef received Speaker CME honoraria from the Georgia Department of Behavioral Health and Developmental Disabilities (DHBDD) and Psychiatric Annals for unrelated CME topics. This study was supported by Behman Hospital.

Contributors: Dr. Nagy Youssef conceptualized and designed the study, wrote the protocol including the electric parameters and titration for LAP-ST, IRB submission, advised and consulted on study implementation, analyzed the data, and wrote the manuscript. Dr. Emad Sidhom helped with the addressing IRB questions and implemented all the study, administered the LAPST as the ECT psychiatrist, collected and entered the data and helped in editing the manuscript. All authors have approved the final article. 
Table 1. Subject demographic and clinical characteristics $(\mathrm{N}=22)$.

\begin{tabular}{|c|c|}
\hline Age, mean (SD), years & $41(15.2)$ \\
\hline Median & 38 \\
\hline Sex, Female, N (\%) & $16(73 \%)$ \\
\hline \multicolumn{2}{|l|}{ Marital Status, N (\%) } \\
\hline Single & $6(31.6 \%)$ \\
\hline Married & $12(63.2 \%)$ \\
\hline Divorced/Widowed & $1(5.2 \%)$ \\
\hline Number of LAP-ST sessions, mean (SD) & $5.7(3)$ \\
\hline Median & 6 \\
\hline Minimum & 1 \\
\hline Maximum & 12 \\
\hline Depressive episode, and did 1 session only, $\mathrm{N}$ & 1 \\
\hline Psychotic episode and did 1 session only, $\mathrm{N}$ & 1 \\
\hline $\begin{array}{l}\text { Primary Psychotic disorders (schizophrenia and } \\
\text { schizoaffective), } \mathrm{N}(\%)\end{array}$ & $11(50 \%)$ \\
\hline Primary Mood Disorders, N (\%) & $11(50 \%)$ \\
\hline \multicolumn{2}{|l|}{ Depressive Episodes: } \\
\hline Unipolar depression, $\mathrm{N}$ & 8 \\
\hline Bipolar depression, $\mathrm{N}$ & 3 \\
\hline
\end{tabular}

*N=19, (3 missing data) 
Table 2. Electric Parameters of first session of LAP-ST, median (IQR) (N=22). ${ }^{*}$

\begin{tabular}{|l|l|}
\hline Current (mA) & $500(500-500)$ \\
\hline Charge (mC) & $48(48-48)$ \\
\hline Dynamic Impedance (Ohm) & $319.5(296.5-389.5)$ \\
\hline Sodium thiopental (mg) & $250(250-250)$ \\
\hline Succinylcholine (mg) & $40(40-40)$ \\
\hline Motor seizures (Sec) & $31(19.3-34)$ \\
\hline EEG seizures (Sec) & $33.5(24.3-36)$ \\
\hline
\end{tabular}

*IQR=Interquartile range 
Table 3. Electric Parameters of Subsequent sessions of LAP-ST, median (IQR) (N= 22). ${ }^{*}$

\begin{tabular}{|l|l|}
\hline Current $(\mathrm{mA})$ & $500(500-512.5)$ \\
\hline Charge $(\mathrm{mC})$ & $288(288-288)$ \\
\hline Dynamic Impedance (Ohm) & $304.8(282-321.25)$ \\
\hline Sodium thiopental (mg) & $250(250-250)$ \\
\hline Succinylcholine (mg) & $40(40-40)$ \\
\hline Motor seizures (min) & \\
\hline EEG (min) & $24.3(18.3-31)$ \\
\hline
\end{tabular}

${ }^{*} \mathrm{QQR}=$ Interquartile range 


\section{REFERENCES}

Al-Rajeh, S., Ogunniyi, A., Awada, A., Daif, A., \& Zaidan, R. (1999). Preliminary assessment of an Arabic version of the Mini-Mental state examination. Ann Saudi Med, 19(2), 150-152. doi:10.5144/0256-4947.1999.150

American Psychiatric Association. (2001). The practice of electroconvulsive therapy: recommendations for treatment, training, and privileging: a task force report of the American Psychiatric Association (2nd ed.). Washington, DC: American Psychiatric Association.

Avramov, M. N., Husain, M. M., \& White, P. F. (1995). The comparative effects of methohexital, propofol, and etomidate for electroconvulsive therapy. Anesth Analg, 81(3), 596-602. doi:10.1097/00000539-199509000-00031

Ayd, F. (1983). Treatment-resistant depression. Intermational Drug Therapy Newsletter, 18, 2527.

Cronholm, B., \& Ottosson, J. O. (1963a). Ultrabrief stimulus technique in electroconvulsive therapy. I. Influence on retrograde amnesia of treatments with the Elther ES electroschock apparatus, Siemens Konvulsator III and of lidocaine-modified treatment. J Nerv Ment Dis, 137(2), 117-123. Retrieved from http://www.ncbi.nlm.nih.gov/entrez/query.fcgi?cmd=Retrieve\&db=PubMed\&dopt=Cita $\underline{\text { tion\&list uids }=14047818}$

Cronholm, B., \& Ottosson, J. O. (1963b). Ultrabrief stimulus technique in electroconvulsive therapy. II. Comparative studies of therapeutic effects and memory disturbances in treatment of endogenous depression with the Elther ES electroshock apparatus and Siemens Konvulsator III. J Nerv Ment Dis, 137(3), 268-276. Retrieved from http://www.ncbi.nlm.nih.gov/entrez/query.fcgi?cmd=Retrieve\&db=PubMed\&dopt=Cita $\underline{\text { tion\&list uids }=14051942}$

Fava, M., \& Davidson, K. G. (1996). Definition and epidemiology of treatment-resistant depression. Psychiatr Clin North Am, 19(2), 179-200. doi:10.1016/S0193-953X(05)702835

Folstein, M. F., Folstein, S. E., \& McHugh, P. R. (1975). "Mini-mental state". A practical method for grading the cognitive state of patients for the clinician. J Psychiatr Res, 12(3), 189198. doi:10.1016/0022-3956(75)90026-6

Fond, G., Bennabi, D., Haffen, E., Brunel, L., Micoulaud-Franchi, J. A., Loundou, A., . . Boyer, L. (2016). A Bayesian framework systematic review and meta-analysis of anesthetic agents effectiveness/tolerability profile in electroconvulsive therapy for major depression. Sci Rep, 6, 19847. doi:10.1038/srep19847

Friedman, E. (1942). Unidirectional electrostimulated convulsive therapy. Am J Psychiatry, 99(2), 218-223. doi:10.1176/ajp.99.2.218 
Griffiths, J. J., Zarate, C. A., Jr., \& Rasimas, J. J. (2014). Existing and novel biological therapeutics in suicide prevention. Am J Prev Med, 47(3 Suppl 2), S195-203.

doi:10.1016/j.amepre.2014.06.012

Husain, M. M., Rush, A. J., Fink, M., Knapp, R., Petrides, G., Rummans, T., . . Kellner, C. H. (2004). Speed of response and remission in major depressive disorder with acute electroconvulsive therapy (ECT): a Consortium for Research in ECT (CORE) report. J Clin Psychiatry, 65(4), 485-491.

Kellner, C. H., Husain, M. M., Knapp, R. G., McCall, W. V., Petrides, G., Rudorfer, M. V., . . . Group, C. P. W. (2016). A novel strategy for continuation ECT in geriatric depression: phase 2 of the PRIDE Study. Am J Psychiatry, 173(11), 1110-1118.

doi:10.1176/appi.ajp.2016.16010118

Kellner, C. H., Husain, M. M., Knapp, R. G., McCall, W. V., Petrides, G., Rudorfer, M. V., . . . Group, C. P. W. (2016). Right unilateral ultrabrief pulse ECT in geriatric depression: phase 1 of the PRIDE Study. Am J Psychiatry, 173(11), 1101-1109. doi:10.1176/appi.ajp.2016.15081101

Kellner, C. H., Knapp, R., Husain, M. M., Rasmussen, K., Sampson, S., Cullum, M., . . Petrides, G. (2010). Bifrontal, bitemporal and right unilateral electrode placement in ECT: randomised trial. Br J Psychiatry, 196(3), 226-234. doi:10.1192/bjp.bp.109.066183

Lee, W. H., Lisanby, S. H., Laine, A. F., \& Peterchev, A. V. (2012). Stimulation strength and focality of electroconvulsive therapy with individualized current amplitude: a preclinical study. Paper presented at the Annual International Conference of the IEEE Engineering in Medicine and Biology Society San Diego, CA. doi: 10.1109/EMBC.2012.6347466. INSPEC Accession Number: 13131273.

Lee, W. H., Lisanby, S. H., Laine, A. F., \& Peterchev, A. V. (2016). Comparison of electric field strength and spatial distribution of electroconvulsive therapy and magnetic seizure therapy in a realistic human head model. Eur Psychiatry, 36, 55-64. doi:10.1016/j.eurpsy.2016.03.003

Levy-Rueff, M., Jurgens, A., Loo, H., Olie, J. P., \& Amado, I. (2008). [Maintenance electroconvulsive therapy and treatment of refractory schizophrenia]. Encephale, 34(5), 526-533. doi:10.1016/j.encep.2007.08.008

Liberson, W. T. (1948). Brief stimulus therapy - physiological and clinical observations. Am J Psychiatry, 105(1), 28-39. doi:10.117/ajp.105.1.28

Lihua, P., Su, M., Ke, W., \& Ziemann-Gimmel, P. (2014). Different regimens of intravenous sedatives or hypnotics for electroconvulsive therapy (ECT) in adult patients with depression. Cochrane Database Syst Rev(4), CD009763. doi:10.1002/14651858.CD009763.pub2

Lisanby, S. H., Luber, B., Schlaepfer, T. E., \& Sackeim, H. A. (2003). Safety and feasibility of magnetic seizure therapy (MST) in major depression: randomized within-subject comparison with electroconvulsive therapy. Neuropsychopharmacology, 28(10), 18521865. doi:10.1038/sj.npp.1300229 
Loo, C. K., Katalinic, N., Smith, D. J., Ingram, A., Dowling, N., Martin, D., . . Schweitzer, I. (2014). A randomized controlled trial of brief and ultrabrief pulse right unilateral electroconvulsive therapy. Int J Neuropsychopharmacol, 18(1). doi:10.1093/ijnp/pyu045

Martin, D. M., Galvez, V., \& Loo, C. K. (2015). Predicting retrograde autobiographical memory changes following electroconvulsive therapy: relationships between individual, treatment, and early clinical factors. Int J Neuropsychopharmacol, 18(12). doi:10.1093/ijnp/pyv067

Matthews, A. M., Rosenquist, P. B., \& McCall, W. V. (2016). Representations of ECT in Englishlanguage film and television in the new millennium. $J$ ect, 32(3), 187-191. doi:10.1097/YCT.0000000000000312

McCall, W. V., Reboussin, D., Prudic, J., Haskett, R. F., Isenberg, K., Olfson, M., . . Sackeim, H. A. (2013). Poor health-related quality of life prior to ECT in depressed patients normalizes with sustained remission after ECT. J Affect Disord, 147(1-3), 107-111. doi:10.1016/j.jad.2012.10.018

McCall, W. V., Rosenquist, P. B., Kimball, J., Haskett, R., Isenberg, K., Prudic, J., . . Sackeim, H. A. (2011). Health-related quality of life in a clinical trial of ECT followed by continuation pharmacotherapy: effects immediately after ECT and at 24 weeks. J ect, 27(2), 97-102. doi:10.1097/YCT.0b013e318205c7d7

Medda, P., Toni, C., Luchini, F., Giorgi Mariani, M., Mauri, M., \& Perugi, G. (2015). Catatonia in 26 patients with bipolar disorder: clinical features and response to electroconvulsive therapy. Bipolar Disord, 17(8), 892-901. doi:10.1111/bdi.12348

Minnai, G. P., Salis, P., Manchia, M., Pinna, M., \& Tondo, L. (2016). What happens to the course of bipolar disorder after electroconvulsive therapy? J Affect Disord, 195, 180-184. doi:10.1016/j.jad.2016.02.027

Murray, C. J., \& Lopez, A. D. (1997). Alternative projections of mortality and disability by cause 1990-2020: Global Burden of Disease Study. Lancet, 349(9064), 1498-1504. doi:10.1016/S0140-6736(96)07492-2

Nahas, Z., Short, B., Burns, C., Archer, M., Schmidt, M., Prudic, J., . . Sackeim, H. A. (2013). A feasibility study of a new method for electrically producing seizures in man: focal electrically administered seizure therapy [FEAST]. Brain Stimul, 6(3), 403-408. doi:10.1016/j.brs.2013.03.004

Noda, Y., Silverstein, W. K., Barr, M. S., Vila-Rodriguez, F., Downar, J., Rajji, T. K., . . . Blumberger, D. M. (2015). Neurobiological mechanisms of repetitive transcranial magnetic stimulation of the dorsolateral prefrontal cortex in depression: a systematic review. Psychol Med, 45(16), 3411-3432. doi:10.1017/S0033291715001609

Palma, M., Ferreira, B., Borja-Santos, N., Trancas, B., Monteiro, C., \& Cardoso, G. (2016). Efficacy of electroconvulsive therapy in bipolar disorder with mixed features. Depress Res Treat, 2016, 8306071. doi:10.1155/2016/8306071 
Peterchev, A. V., Krystal, A. D., Rosa, M. A., \& Lisanby, S. H. (2015). Individualized low-amplitude seizure therapy: minimizing current for electroconvulsive therapy and magnetic seizure therapy. Neuropsychopharmacology, 40(9), 2076-2084. doi:10.1038/npp.2015.122

Peterchev, A. V., Rosa, M. A., Deng, Z. D., Prudic, J., \& Lisanby, S. H. (2010). Electroconvulsive therapy stimulus parameters: rethinking dosage. J ect, 26(3), 159-174. doi:10.1097/Yct.0b013e3181e48165

Prudic, J., \& Sackeim, H. (1999). Electroconvulsive therapy and suicide risk. J Clin Psychiatry, 60 Suppl 2, 104-110.

Rosa, M. A., Abdo, G. L., Lisanby, S. H., \& Peterchev, A. (2011). Seizure induction with lowamplitude-current (0.5 A) electroconvulsive therapy. J ect, 27(4), 341-342. doi:10.1097/YCT.0b013e31822149db

Rush, A. J., Trivedi, M. H., Wisniewski, S. R., Nierenberg, A. A., Stewart, J. W., Warden, D., . . Fava, M. (2006). Acute and longer-term outcomes in depressed outpatients requiring one or several treatment steps: a STAR*D report. American Journal of Psychiatry, 163(11), 1905-1917. doi:10.1176/appi.ajp.163.11.1905

Sackeim, H. A., Luber, B., Moeller, J. R., Prudic, J., Devanand, D. P., \& Nobler, M. S. (2000). Electrophysiological correlates of the adverse cognitive effects of electroconvulsive therapy. J ect, 16(2), 110-120. Retrieved from https://www.ncbi.nlm.nih.gov/pubmed/10868321

Sackeim, H. A., Portnoy, S., Neeley, P., Steif, B. L., Decina, P., \& Malitz, S. (1986). Cognitive consequences of low-dosage electroconvulsive therapy. Ann N Y Acad Sci, 462, 326-340. doi:10.1111/j.1749-6632.1986.tb51267.x

Sackeim, H. A., Prudic, J., Devanand, D. P., Kiersky, J. E., Fitzsimons, L., Moody, B. J., ... . Settembrino, J. M. (1993). Effects of stimulus intensity and electrode placement on the efficacy and cognitive effects of electroconvulsive therapy. N Engl J Med, 328(12), 839846. doi:10.1056/NEJM199303253281204

Sackeim, H. A., Prudic, J., Devanand, D. P., Nobler, M. S., Lisanby, S. H., Peyser, S., . . Clark, J. (2000). A prospective, randomized, double-blind comparison of bilateral and right unilateral electroconvulsive therapy at different stimulus intensities. Arch Gen Psychiatry, 57(5), 425-434. doi:10.1001/archpsyc.57.5.425

Sackeim, H. A., Prudic, J., Nobler, M. S., Fitzsimons, L., Lisanby, S. H., Payne, N., . . Devanand, D. P. (2008). Effects of pulse width and electrode placement on the efficacy and cognitive effects of electroconvulsive therapy. Brain Stimulat, 1(2), 71-83. doi:10.1016/j.brs.2008.03.001

Sienaert, P. (2016). Based on a true story? The portrayal of ECT in international movies and television programs. Brain Stimul, 9(6), 882-891. doi:10.1016/j.brs.2016.07.005

Sobin, C., Sackeim, H. A., Prudic, J., Devanand, D. P., Moody, B. J., \& McElhiney, M. C. (1995). Predictors of retrograde amnesia following ECT. Am J Psychiatry, 152(7), 995-1001. doi:10.1176/ajp.152.7.995 
Squire, L. R. (1977). ECT and memory loss. Am J Psychiatry, 134(9), 997-1001. doi:10.1176/ajp.134.9.997

Squire, L. R., \& Zouzounis, J. A. (1986). ECT and memory: brief pulse versus sine wave. Am J Psychiatry, 143(5), 596-601. doi:10.1176/ajp.143.5.596

Squire, S. R., \& Slater, P. C. (1978). Bilateral and unilateral ECT: effects on verbal and nonverbal memory. Am J Psychiatry, 135(11), 1316-1320. doi:10.1176/ajp.135.11.1316

Subedi, S., Aich, T. K., \& Sharma, N. (2016). Use of ECT in Nepal: A one year study from the country's largest psychiatric facility. J Clin Diagn Res, 10(2), VC01-VC04. doi:10.7860/JCDR/2016/14660.7269

Szkultecka-Debek, M., Miernik, K., Stelmachowski, J., Jakovljevic, M., Jukic, V., Aadamsoo, K., . . . Augustynska, J. (2016). Schizophrenia causes significant burden to patients' and caregivers' lives. Psychiatr Danub, 28(2), 104-110. Retrieved from http://www.ncbi.nlm.nih.gov/pubmed/27287783

Tharyan, P., \& Adams, C. E. (2005). Electroconvulsive therapy for schizophrenia. Cochrane Database Syst Rev(2), CD000076. doi:10.1002/14651858.CD000076.pub2

UK ECT Review Group. (2003). Efficacy and safety of electroconvulsive therapy in depressive disorders: a systematic review and meta-analysis. Lancet, 361(9360), 799-808. doi:10.1016/S0140-6736(03)12705-5

Weiner, R. D. (1980). ECT and seizure threshold: effects of stimulus wave form and electrode placement. Biol Psychiatry, 15(2), 225-241. Retrieved from http://www.ncbi.nlm.nih.gov/entrez/query.fcgi?cmd=Retrieve\&db=PubMed\&dopt=Cita tion\&list uids $=7417613$

Youssef, N. A., \& McCall, W. V. (2014). Relapse prevention after index electroconvulsive therapy in treatment-resistant depression. Ann Clin Psychiatry, 26(4), 288-296. Retrieved from http://www.ncbi.nlm.nih.gov/pubmed/25401716 
Chapter 13 General Discussion 


\section{GENERAL DISCUSSION}

\subsection{Summary of key findings}

This thesis addresses several questions in the field of epigenetics and brain stimulation in order to provide a step forward in furthering our understanding in each of these areas. Much work needs to be done, but we hope that this work not only gets us closer to advancing these fields, but also sheds light on the important questions in these fields, which will guide the reader to the next steps of inquiry.

Section I, Chapter 2 started with a discussion of the definition of epigenetics and goes on to systematically review and explore the studies in the literature related to the association between epigenetics and depression and/or suicide. Based on pre-specified terms and criteria, sixteen studies met the inclusion criteria. I found that epigenetic changes seem to have associations with both depression and suicide. Several studies showed that hypermethylation of Brain-derived neurotrophic factor (BDNF) is specifically involved in suicide. Tropomycin receptor kinase $B$ (TrkB) hypermethylation was also shown to be associated with suicide in several studies. Future research is needed in a larger sample to further characterize and replicate these changes.

Chapter $\mathbf{3}$ explores the impact of trauma and PTSD beyond the affected individual. It not only examines the possibility that psychological trauma can affect the biology of the individual (by review of the current literature), but also that epigenetic alterations related to trauma might be passed on to offspring. Thus, the main aim of this chapter is to review the emerging literature on evidence of transgenerational inheritance via DNA methylation in humans due to trauma exposure. This chapter found an accumulating amount of evidence of an enduring effect of trauma exposure being passed down to offspring transgenerationally via DNA methylation alterations, which has the capacity to (epigenetically) change the expression of genes and the metabolome. This chapter provides an overview of where we stand in this area and a clearer view of where we should go in terms of future research directions.

To overcome an important limitation of the reviewed studies in chapters 2 and 3 (and a common limitation in the field of epigenetics in general), which is that epigenetic studies are commonly underpowered, Chapter 4 presents a combined effort by creating a consortium for detecting epigenetic markers in PTSD. This consortium has led to the publications of combined analysis of over 34 centers. Here, we describe the purpose and methods of this international consortium that was developed to promote collaborative studies on the role of epigenetics in PTSD. We brought together investigators representing seven cohorts (total $N=1147$ ) that include detailed information on trauma exposure, PTSD symptomatology, and genome-wide DNA methylation. The objective of this consortium was to increase the analytical sample size by pooling data and combining expertise, in order to identify the DNA methylation patterns associated with PTSD. Several quality control and analytical pipelines were evaluated for their control of genomic inflation and technical artifacts, and a joint analysis procedure was established to derive comparable data over the cohorts for meta-analysis. The proposed ways help with dealing with type I error inflation and discuss the advantages and disadvantages of applying robust error estimates. To evaluate our pipeline, we report results from an EpigenomeWide Association Study (EWAS) of age, which is a well-characterized phenotype with known 
epigenetic associations. Overall, while EWAS are highly complex and subject to similar challenges as Genome-Wide Association Studies (GWAS), we demonstrated that an epigenetic metaanalysis can be well-powered to identify epigenetic associations. This study can be used as a framework for consortium efforts for EWAS.

Epigenetics may also play a role in resilience against mental illness. Moving from the molecular level to the clinical level as it relates to resilience, Chapter $\mathbf{5}$ presents a 3-year longitudinal clinical observation study in U.S. military veterans to evaluate the correlation and role of resilience and resilience factors in predicting suicidal ideation and attempts. In this study of 178 veterans, we found that higher resilience at baseline predicted lower suicidality at followup, suggesting a protective effect for resilience. Within the specific domains of resilience, secure relationships and positive acceptance of change significantly predicted lower suicidality. The findings indicate the importance of developing programs to boost resilience as part of clinical care or as preventative efforts.

Chapter 6 examines if there are racial differences to the effect of childhood trauma, and how resilience influences the effect of adverse childhood experiences (ACE) on the development of depressive symptoms. This is an area that is much less studied. The specific aims of this study were to examine: 1) whether there is a dose-response relationship between trauma and depressive symptoms; 2) whether early trauma affects European Americans (EA) and African Americans (AA) in a similar fashion; and 3) whether resilience mitigates the effect of trauma.

This was a cross-sectional study of 413 subjects who were 19 years or older. ACEs were significantly associated with depression severity in a dose-response fashion $(p<0.001)$. Notably, AAs had lower depression scores at low to moderate levels of ACEs than EAs but reported comparable levels of depression with severe exposure to ACEs ( $p$ interaction $=0.05$ ). In both EAs and AAs participants, those with high and medium levels of resilience showed less depressive symptoms when compared to those with low resilience $(p<0.05)$.

Despite the limitation that this was a cross-sectional study, it provided initial evidence to be examined in a longitudinal study that higher resilience mitigated the impact of childhood adversities on depressive symptoms in young adults. In addition, the study points to the racial difference in resilience to $A C E$, which points to the biological component of resilience as a likely candidate.

In order to examine other non-biological factors that can possibly moderate the effect on either making individuals more vulnerable to developing PTSD or to boost resilience, we performed a cross-sectional study to examine the relationship between moral injury and PTSD, and the impact of religious involvement (RI) on that relationship presented in Chapter 7. Religiosity and other moral values are used by some individuals to boost resilience. Traumatic experiences can cause ethical conflicts. Moral injury has been used to describe this emotional/cognitive state and may either contribute to the development of PTSD or block its recovery. This study of 120 veterans found a strong relationship between moral injury and PTSD symptoms ( $r=0.54, p<0.0001)$, and between moral injury and $R I(r=-0.41, p<0.0001)$, but only a weak relationship was found between RI and PTSD symptoms ( $r=-0.17, p=0.058)$. RI did not mediate or moderate the relationship between moral injury and PTSD symptoms in the overall sample. However, among veterans who were in non-Middle Eastern conflicts, a significant 
buffering effect of religiosity was found. The explanation is not clear, but may be due to age differences with different adherences to religious values.

\section{Section II:}

While shifting from the epigenetics to the brain stimulation section, Chapter 8 reviews pertinent studies aimed to avoid relapse and recurrence of depressive symptoms. An acute course of ECT can lead to remission of depressive symptoms in a substantial portion of treatmentresistant patients, but many patients relapse after the ECT course. Thus, this chapter reviews the studies of continuation ECT to examine how to keep patients doing well. The chapter reviews published clinical trials until April 2014. Most studies suggest that combined continuation of ECT and continuation of pharmacotherapy are more effective together than either alone for preventing relapse. Of interest, this work was published prior to the multicenter randomized clinical trial: Prolonged Remission in Depressed Elderly (PRIDE) and has come to the same conclusions as the PRIDE clinical trial. This systematic review found that combined continuation of pharmacotherapy plus ECT is better than either alone.

Although Chapter 8 provides important insights regarding symptom remission and relapse prevention, symptom remission is not sufficient for individuals' well-being and functioning. Improvement in the well-being and functioning of patients is achieved by improvement in health-related quality of life (HRQOL), which is also important in its own right. Thus, in Chapter 9, we analyzed data from the PRIDE study to examine whether ECT plus medications ("STABLE + PHARM" group) had superior HRQOL compared with medications alone ("PHARM" group) as a continuation strategy after successful acute right unilateral ECT for major depressive disorder (MDD). The PHARM group included venlafaxine plus lithium. We hypothesized that scores from the Medical Outcomes Study Short Form-36 (SF-36) would be higher during continuation treatment in the "STABLE + PHARM" group versus the "PHARM" group. Remitters to the acute course of ECT were randomized to 6 months of "STABLE + PHARM" versus "PHARM." One hundred twenty patients $\geq 60$ years old were included. The "STABLE + PHARM" received the same medications (venlafaxine and lithium), plus 4 weekly outpatient ECT sessions with additional ECT sessions as needed. "STABLE + PHARM" group had a 7-point advantage $(3.5-10.4,95 \% \mathrm{Cl})$ for Physical Component Score of SF-36 ( $\mathrm{P}<0.0001)$, and an 8.2-point advantage $(4.2-12.2,95 \% \mathrm{Cl})$ for Mental Component Score $(\mathrm{P}<0.0001)$. Additional ECT resulted in overall net health benefit. Consideration should be given to administration of additional ECT to prevent relapse during the continuation phase of treatment of MDD.

Although depressive episodes are the most common indication for ECT in the United States (Patel, Sreeram, Thakur, Bachu, \& Youssef, 2019), there are several other potential indications that are still under investigation for ECT such as PTSD (Chapter 11) and novel techniques of administration such as LAP-ST (Chapter 12). Chapter 10, as its title indicates, provides a charting of "the ECT Course: Where have we been? Where are we headed?" More specifically, it discusses and reviews the current knowledge of ECT in general, while discussing several indications as well as patient selection, choice of electrode placement and stimulus parameters, augmentation with pharmacotherapy, and the use of continuation/maintenance treatment. Then, it discusses future lines of investigations in ECT including research into 
biomarkers and neuroplasticity related to ECT response, as well as new, investigational methods of ECT delivery.

Chapter 11 switches gears from some of the well-established indications for ECT to an investigational indication of ECT, namely treatment-resistant PTSD. There is little to offer patients who do not respond to standard interventions of psychotherapy and pharmacotherapy for PTSD. Thus, the objective of this chapter is to systematically review human data on whether ECT is effective in PTSD. The systematic literature review was performed through August 2016 for clinical studies and case reports examining the efficacy of ECT for PTSD symptoms. The literature search generated 3 retrospective studies, 1 prospective uncontrolled clinical trial, and 5 case reports. Given the small sample size and lack of a large randomized trial, it was not clear whether favorable outcomes were attributed to improvement in depression (as opposed to core PTSD symptoms) given that most patients underwent ECT for depression as well. Thus, current efficacy data are inconclusive on the effects of ECT on PTSD symptoms. Randomized controlled trials are necessary to examine the use of ECT in medication-refractory PTSD patients with and without comorbid depression. Subsequent studies may address response in PTSD subtypes and the use of novel techniques such as memory reactivation before ECT.

Chapter 12 focuses on another form of investigational trial in humans. I conceptualized a proof of concept (POC) trial to examine if low current pulse amplitude (500-600mA) used to induce seizures during a full acute course in humans can retain efficacy while reducing cognitive side effects of ECT. The standard clinical current amplitude of $800-900 \mathrm{~mA}$ may be too high, thus driving the electric field deeper to areas responsible for memory and cognitive side effects such as the hippocampus and temporal lobe. These cognitive side effects of ECT can discourage some patients indicated for ECT from receiving ECT and also affect some others after an acute course of ECT. I have called this technique Low Pulse Amplitude Seizure Therapy (LAP-ST) given that seizures (not convulsions) are the active ingredient needed for therapeutic response (even if the convulsions are blocked by a muscle relaxant). Reducing pulse amplitude has been shown in computer models to improve focality of the electric field, thus mitigating cognitive side effects. Thus, Chapter 12 examines in a POC open-label trial the feasibility, safety, and cognitive side effects of LAP-ST, if any.

Patients with treatment-resistant primary mood disorders (in depressive episodes), or psychotic disorders who were clinically indicated to undergo ECT were offered to be enrolled. The study consisted of a full acute course of LAP-ST. The primary outcome was feasibility of seizure induction. Clinical outcome measures were: time to reorientation (TRO), Mini Mental State Examination, Montgomery-Åsberg Depression Rating Scale, Brief Psychiatric Rating Scale, and Clinical Global Impression Scale. Twenty-two patients consented for enrollment. We found that LAP-ST is feasible with a quick median Time to Re-Orientation (TRO) of 4.5 minutes (TRO being the best acute predictor of memory and cognitive side effects as shown in several studies). Treatment was efficacious for both depressive and psychotic symptoms. These results, although preliminary, suggest that the LAP-ST technique may result in less cognitive side effects with comparable efficacy when compared to the standard ECT techniques. 


\subsection{Limitations \& Challenges}

Some of the important limitations encountered in epigenetics studies are described in the individual chapters. This thesis, (and the epigenetic studies it is based on), also have some of these limitations. One of the most important limitations may be the small to moderate sample size of many studies in epigenetics, limiting the power to detect significant changes. It is very difficult for most centers to gather a sufficiently large sample size given the huge participant numbers as well as the financial resources needed. Thus, it is crucial for the field to collaborate in organized consortia and pool data to generate sufficient power to detect actual differences and to perform replication studies.

Another limitation inherent to epigenetic studies is their interpretability of causality rather than associations. When associations are found, it is still unknown if the found epigenetic dysregulation caused either disease or resilience, whether it is a consequence, or a mere epiphenomenon. Thus, once a replicable association is found, more focused research is needed to unravel these issues. Disentangling these processes will require more longitudinal studies.

A third difficulty that can be a limitation of certain epigenetic studies is the dynamic nature of epigenetic regulation. This can take many forms including the possible observer effect known in quantum mechanics: where the mere act of observing might change the findings, i.e. the experimental procedure itself may result in epigenetic changes. For instance, while observing the behavioral impact of epigenetic alterations in an experiment, behavioral testing in that very experiment in animal models may be stressful enough to induce epigenetic changes (Stankiewicz, Swiergiel, \& Lisowski, 2013). It has also been shown that repeated testing can induce epigenetic changes related to learning and memory [2].

Fourthly, limitations in the tools used and cell-type specificity can mislead us. For example, proxy samples are used (like blood or saliva) for brain-mediated disorders. However, cell-type specifically (Davies et al., 2012) may limit the data extracted from proxy sites. Since sampling brain tissue in living individuals cannot be done for this purpose, this makes it a hard dilemma to solve. We hope more correlation studies between brain and peripheral samples can be done (Hannon, Lunnon, Schalkwyk, \& Mill, 2015; Smith et al., 2015) to try to get us as close as possible to the needed answer.

Regarding limitation of the brain stimulation studies, they are described in the individual chapters. However, we would like to add here that important limitations in the field relevant to ECT and general brain stimulation studies are that we still lack some important knowledge of the best combination of parameters to use to optimize efficacy and minimize side effects. There is still a need for studies that examine this further. Some of these questions may need to be examined first in animal models as a platform to optimize the parameters. This may apply to ECT as well as other modalities such as transcranial magnetic stimulation and deep brain stimulation in terms of optimizing which combination of parameters to be used in each case.

Another limitation is the lack of enough predictors and precision medicine variables in the field of ECT (and brain stimulation in general). Predictors and precision treatments are important areas for future studies. 


\subsection{Future perspectives}

Future perspectives of research would be to address the above limitations and challenges in the field by performing large-scale, concerted longitudinal studies encompassing multi-level variables in the biological, psychological, social and spiritual domains. Given the early phase of studies and the explorative nature of most epigenetic studies, it would also be important to reproduce relevant findings in independent cohorts and across people with different backgrounds.

Some other unique future directions that constitute an overlap and interaction between the field of epigenetics and the field of brain stimulation are also worth concluding and are outlined below.

\section{Next steps after the current work related to the overlap and interaction between the} fields of epigenetics and brain stimulation:

Although the two main sections of this thesis, namely brain stimulation on one side and resilience and epigenetics on the other side, are not always related, some preliminary studies in the literature have examined the relationship and interactions between brain stimulation modalities and epigenetics and between epigenetics and resilience. Although these studies are preliminary, there is enough evidence to suggest some validity in the interactions depicted in Figures 1 and 2, suggesting that brain stimulation interventions may lead to epigenetic changes. However, I believe that future research is much needed in these areas. I hope that this thesis has helped answer some of the needed questions and brought our understanding a step forward. In addition, I hypothesize that brain stimulation interventions might improve resilience (some support of this hypothesis comes from preliminary data from our lab not discussed here). These relations might be mediated by epigenetics (see Figure 1). These are very important areas to be explored further in the future.

Figure 1: Mediation model.

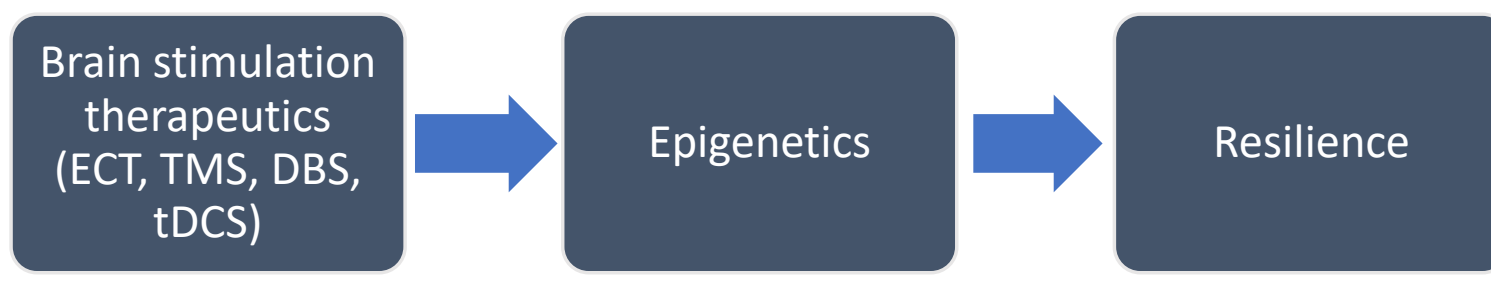

Mediation model: Epigenetics mediate the relationship between brain stimulation modalities and resilience. Brain stimulation may cause epigenetic alterations, which in turn would boost resilience (Youssef. Brain stimulation, epigenetics and resilience, submitted).

Figure 2: Predictor model 


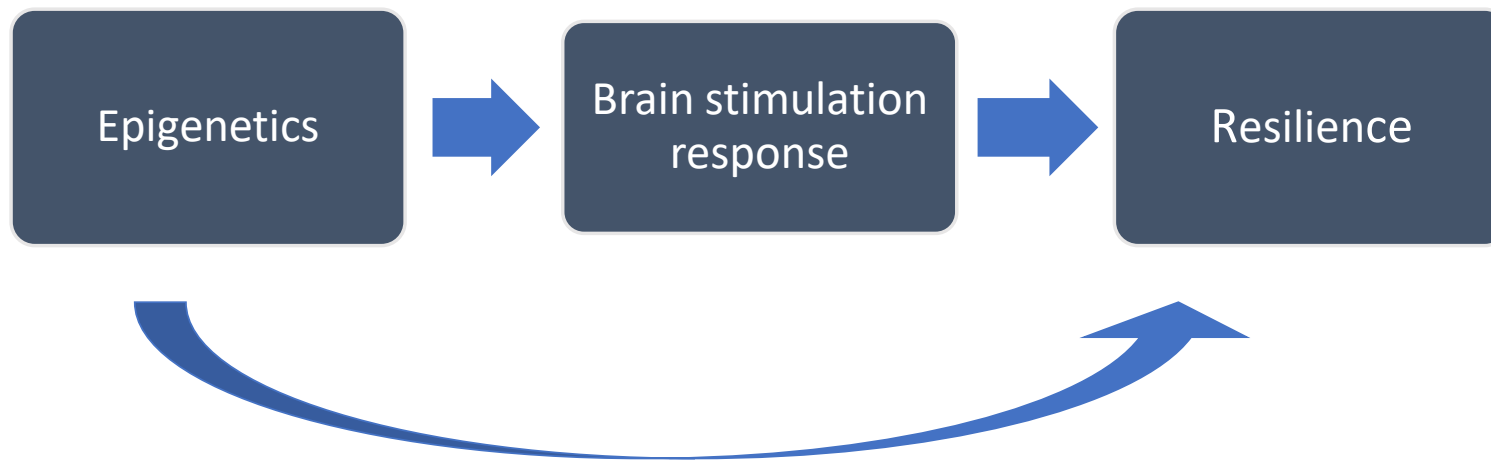

Predictor model: Epigenetics markers may predict treatment response or remission from brain stimulation therapeutics with no direct correlation with resilience. In addition, epigenetics might predict repose to brain stimulation intervention and in turn, brain stimulation intervention might improve resilience (Youssef. Brain stimulation, epigenetics and resilience, submitted).

On a related front, epigenetics' signature might provide markers of treatment response for brain stimulation interventions (see Figure 2). If this predictor relationship pans out, it could have very important clinical value and implications. Clinically, a simple blood test or saliva swab (if proven to correlate enough with target brain areas) could differentiate the group of patients who would benefit from a course of ECT, for instance, and the group who would not benefit. This also could be an important field that should be further explored in the future.

Another area of future direction, as described in Youssef 2018 is depicted in Figure 3 (Youssef, 2018). I hypothesized that clearance of traumatic and/or PTSD-related memories might be induced after provoking traumatic memories, then giving an ECT stimulus. This may work, at least in part, via induction of neurogenesis. A possible way this may happen is either via a direct electric stimulation inducing neurogenesis or via an epigenetic mechanism inducing neurogenesis (Youssef, 2018), which may in turn help clear traumatic memories and increase the resilience threshold. One demonstration that may hold relevance to solving this mystery is seen in the results of a recent study published in Nature demonstrating that hippocampal adult neurogenesis confers resilience towards stress (Anacker et al., 2018).

As indicated elsewhere, traumatic memories "generate some of the most persistent memories and are the hardest to erase. This might be explained by the cellular mechanism for encoding durable memories" (Youssef, 2018) possibly mediated via epigenetic alterations (Hao, Youssef, Davis, \& Su, 2018). 
Figure 3. Proposed mechanism for clearance of traumatic memories and neurogenesis.

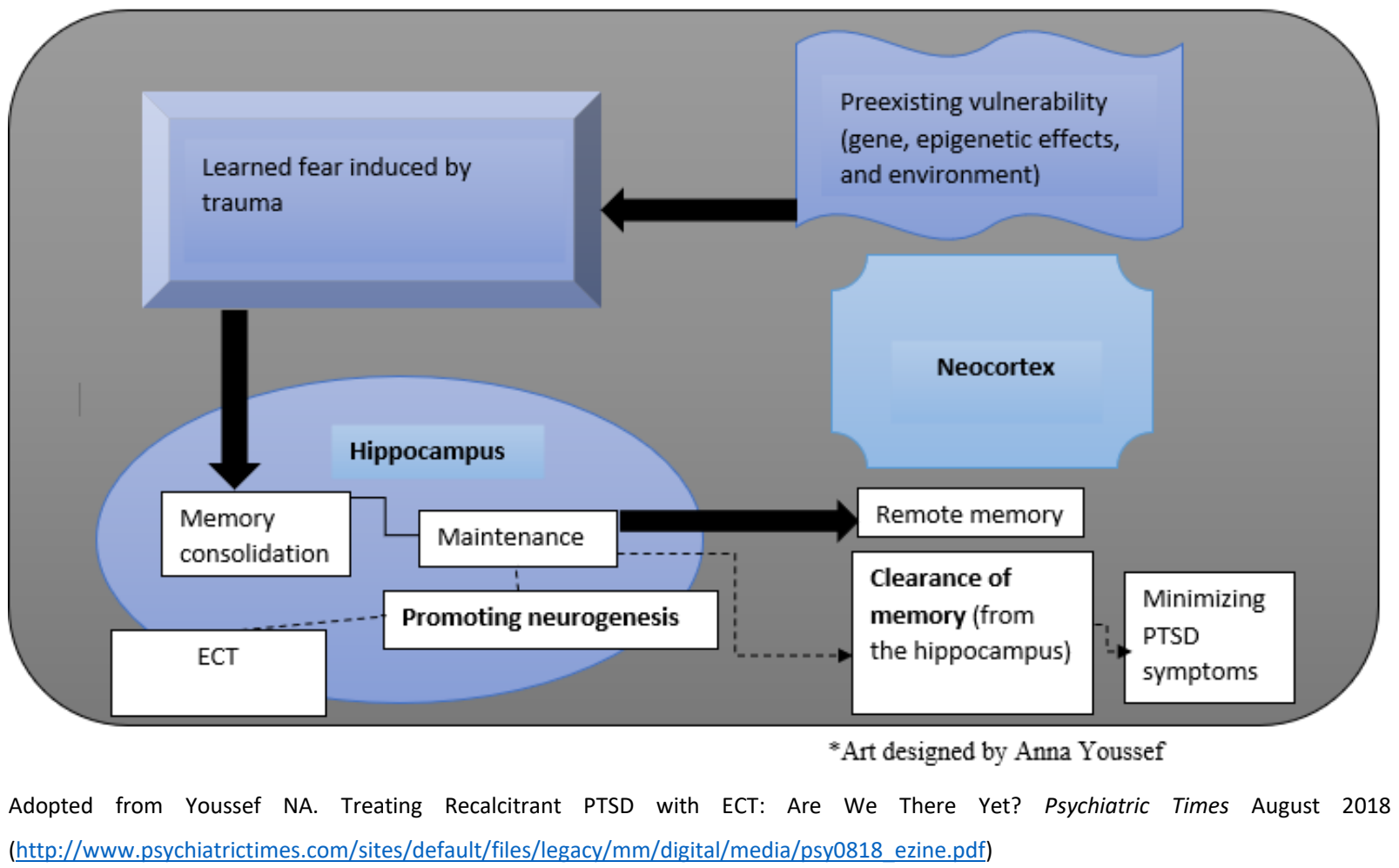

I hope the body of work in this thesis spurs more research on the relationship between epigenetics and brain stimulation in different laboratories around the world. Moreover, my next steps in my translational research are to investigate these relationships given my interest, prior work, and experience in these areas.

Among several areas, two concrete and immediate areas of future directions of research after my PhD that I plan to investigate (based on my work in the areas of brain stimulation and epigenetics) are as follows:

1. How ECT may improve resilience? Also, if our initial pilot data are confirmed in a larger study, can epigenetics be the (or one of the) mechanistic explanation(s)?

2. Investigate epigenetics' signature or markers of treatment response for ECT. This has important clinical implications, and will help us toward individualized health care.

This of course would be a rich area for collaboration with colleagues and with experts from both the field of epigenetics and that of ECT/brain stimulation. It would also be welcomed for other labs to simultaneously explore this over the next several years, as these questions can have an important impact on the field and on patients' lives. 


\section{REFERENCES}

Anacker, C., Luna, V. M., Stevens, G. S., Millette, A., Shores, R., Jimenez, J. C., . . Hen, R. (2018). Hippocampal neurogenesis confers stress resilience by inhibiting the ventral dentate gyrus. Nature, 559(7712), 98-102. doi:10.1038/s41586-018-0262-4

Davies, M. N., Volta, M., Pidsley, R., Lunnon, K., Dixit, A., Lovestone, S., . . Mill, J. (2012). Functional annotation of the human brain methylome identifies tissue-specific epigenetic variation across brain and blood. Genome Biol, 13(6), R43. doi:10.1186/gb2012-13-6-r43

Hannon, E., Lunnon, K., Schalkwyk, L., \& Mill, J. (2015). Interindividual methylomic variation across blood, cortex, and cerebellum: implications for epigenetic studies of neurological and neuropsychiatric phenotypes. Epigenetics, 10(11), 1024-1032. doi:10.1080/15592294.2015.1100786

Hao, G., Youssef, N. A., Davis, C. L., \& Su, S. (2018). The role of DNA methylation in the association between childhood adversity and cardiometabolic disease. Int J Cardiol, 255, 168-174. doi:10.1016/j.ijcard.2017.12.063

Patel, R., Sreeram, V., Thakur, T., Bachu, R., \& Youssef, N. (2019). A national longitudinal study for regional variation of inpatient ECT utilization from 4411 hospitals across the United States Brain Stimul, 12(2), 398-399. doi:10.1016/j.brs.2018.12.283

Smith, A. K., Kilaru, V., Klengel, T., Mercer, K. B., Bradley, B., Conneely, K. N., . . Binder, E. B. (2015). DNA extracted from saliva for methylation studies of psychiatric traits: evidence tissue specificity and relatedness to brain. Am J Med Genet B Neuropsychiatr Genet, 168b(1), 36-44. doi:10.1002/ajmg.b.32278

Stankiewicz, A. M., Swiergiel, A. H., \& Lisowski, P. (2013). Epigenetics of stress adaptations in the brain. Brain Res Bull, 98, 76-92. doi:10.1016/j.brainresbull.2013.07.003

Youssef, N. A. (2018). Treating recalcitrant PTSD with ECT: are we there yet? Psychiatric Times, August. Retrieved from http://www.psychiatrictimes.com/sites/default/files/legacy/mm/digital/media/psy0818 ezine.pdf 


\section{Valorization of Knowledge}

Nagy A. Youssef

Affective disorders, including major depressive disorder (MDD), are a leading cause of disability worldwide with a high burden of morbidity and mortality (World Health Organization, 2017). Affective disorders are also a major risk factor for mortality, including both death due to suicide and other medical conditions.

Affective disorders and depressive episodes have long been recognized to result from an interplay between environmental and biological factors (Wankerl et al., 2014). Epigenetics involves functional modifications of genes that are affected by environmental factors. Thus, epigenetics is likely an important mechanism that contributes to the development of and/or relapse from affective disorders. Several studies showed that certain epigenetic changes (mainly hypermethylation) to be associated with suicide and depression.

Treatment-resistant depression (TRD) is defined as MDD that is not responsive to treatment despite two different antidepressant regimens of adequate duration and dosage; 1 out of 3 patients suffering from MDD has TRD (European Medicines Agency, 2013; Kular \& Kular, 2018; U.S. Department of Health and Human Services, 2018 ). Many patients who do not respond to medications and psychotherapy may have a robust response and remission to Electroconvulsive Therapy (ECT).

Another stress related disorder is Posttraumatic Stress Disorder (PTSD), which also causes substantial burden and suffering (American Psychiatric Association, 2013; Department of Veterans Affairs and Department of Defense, 2017). PTSD has a high prevalence in many countries, and is more prevalent in combat veterans representing up to $17 \%$ (American Psychiatric Association, 2013; Department of Veterans Affairs and Department of Defense, 2017; Kessler, Chiu, Demler, Merikangas, \& Walters, 2005; Miao, Chen, Wei, Tao, \& Lu, 2018). PTSD has a strong genetic component, evidenced by numerous research studies. For instance, twin studies found estimated heritability ranging from 30\% to as high as $72 \%$ (Sartor et al., 2011; Stein, Jang, Taylor, Vernon, \& Livesley, 2002). Transgenerational studies done with children of holocaust survivors show that PTSD is more likely to occur in certain families, for review see e.g. Youssef et al. (Youssef, Lockwood, Su, Hao, \& Rutten, 2018). However, PTSD etiologically involves multifactorial processes and a dynamic and complex interplay of different biological systems and psychological trauma (Ratanatharathorn et al., 2017). Even though it is known that PTSD is heritable, the complex underlying genetic architecture of this disorder is unknown (Ratanatharathorn et al., 2017).

Within the nature-nurture discussions, scholars have proposed to study the complex interaction between genetics and the environment in stress-related mental disorders (depression and PTSD) using epigenetics. Similar research has proven fruitful in the field of cancer genomics. This approach may in fact be uniquely suited to be applied to PTSD research, since the effect of trauma on mental health is suspected to be mediated through epigenetic changes. Consequently, there is growing appreciation of the role of epigenetics in the etiology of PTSD. 
Potential applications of the knowledge gained for future research of stress related disorders.

A. Progress in the scientific areas of research of epigenetics and resilience can advance our understanding of the mechanism underlying these stress-related mental disorders and on the other hand may innovate treatment modalities to manage these disorders. The research progress may thereby assist in several manners in the following 2 ways:

1) Sub-classifying PTSD and depressive episodes of mood disorders with the help of epigenetic biological markers. This can improve possibilities in personalizing diagnosis and treatment interventions (Feng \& Youssef, 2019).

2) Epigenetics plays an important role in improving our understanding of the interplay between environment and underlying genetics, and identifying mediating biological processes, which may be targeted for modulation by interventions, such as medication, lifestyle and dietary components.

B. Also, based on the findings of this thesis, the identification of factors and mechanisms involved in co-determining and/or mediating differential susceptibility and resilience to the effects of trauma and other exposures may enable novel preventative and treatment modalities for stress-related disorders. Moreover, brain stimulation interventions also can play important roles in the treatment of stress disorders especially the most powerful of these interventions, namely ECT (Lisanby, 2007; Rush et al., 2006).

Potential applications of the knowledge gained for improving patient care and translational research to prevent and/or treat stress-related disorders: ${ }^{*}$

Some studies have already identified epigenetics markers that can be tested clinically and can be used in clinical care not too far in the future. This can, for instance, help in attempts for predicting inter-individual differences in response to treatment modalities, i.e. personalized medicine.

Precision medicine can benefit both the patient and clinician in terms of saving suffering, money, time, and frustration (Feng \& Youssef, 2019). Epigenetic biomarkers have been suggested (at least in some initial studies) to predict response to pharmacological treatment in depression (Goud Alladi, Etain, Bellivier, \& Marie-Claire, 2018). Promising studies found that hypomethylation of genes related to the serotonin transporter (Domschke et al., 2014) and receptor (Wang et al., 2018) predicted impaired response to escitalopram. Also, another study found hypermethylation at a different gene, the IL-11 gene promoter, predicted favorable response to escitalopram and decreased response to nortriptyline (Powell et al., 2013). However, in order to reduce false-positive findings, the field warrants adequately powered studies with an agnostic, genome-wide approach and using a discovery as well as a replication approach.

Regarding epigenetics and ECT, data have both suggested epigenetic modifications by ECT in animals, and on a different front, initial human data examined epigenetics as biomarkers of treatment response (Feng \& Youssef, 2019). Initial data of epigenetic modifications produced by electroconvulsive seizures (ECS) has been presented by a review paper (de Jong et al., 2014). This paper reviewed all rodent studies. All studies in the review have multiple epigenetic effects that are suggested to modulate the therapeutic effects of ECT. This included increased histone acetylation in c-Fos, BDNF, and CREB genes, demethylation of the BDNF promoter, and changes in levels of various micro-RNAs. 
Initial human studies also examined epigenetics as predictors of treatment response for ECT (Kleimann et al., 2015), and was further supported by a follow-up study (Neyazi et al., 2018). The initial human study found that both ECT remitters and responders had significantly lower baseline methylation percentage in the BDNF promoter when compared to non-responders (Kleimann et al., 2015). They proposed the potential application of BDNF promoter methylation as a predictive biomarker for ECT. The follow-up study examined the methylation of the promoter for p11, (a molecule involved in serotonin signaling and upregulation of BDNF) (Neyazi et al., 2018). The researcher studied p11 promoter methylation in a mouse model of depression, and then did a translational proof-of-concept study in humans.

In mice, ECS responders showed hypermethylation of p11 promoter and increased p11 mRNA expression in the PFC. Patients undergoing ECT showed similar findings in serum samples at all-time points, and patients with higher baseline p11 promoter methylation had significantly less depression after ECT treatment. The results suggest that p11 methylation can predict ECT response, which was tested (Feng \& Youssef, 2019).

In a separate patient sample, p11 promoter methylation above $72.15 \%$ predicted response to ECT with a positive predictive value (PPV) of $90 \%$. The test identified responders with a sensitivity of $70 \%$ and specificity of $73 \%$.

This line of inquiry seems promising, and if pursued, can lead to fruitful epigenetic biomarkers of treatment response and precision psychiatry (Yao et al., 2019).

Further studies should also replicate the information in this thesis regarding that hypermethylation of brain-derived neurotrophic factor (BDNF) and tropomycin receptor kinase B (TrkB) hypermethylation are potential biomarkers for suicide. This could, if replicated, be used as a biomarker to help in focused efforts of suicide prevention.

Similarly, further replication studies should examine the accumulating evidence suggesting enduring effects of trauma exposure being passed down to offspring transgenerationally via DNA methylation alterations. This can inform and direct further public health efforts in preventing traumatic child abuse and partner violence and boosting resilience among other programs and initiatives.

\section{Policy, stakeholders, and society:}

As mentioned above, epigenetic research can provide both mechanistic understanding and biomarkers of treatment response. Policy makers should improve and allocate funding for the development of epigenetic research and support epigenetic consortia to increase sample size and for the statistical power needed for such research, which involves many multiple comparisons and thousands to millions of variables. We have demonstrated such an example in our collective efforts in developing Epigenome-Wide Consortium in PTSD. We demonstrated that epigenetic meta-analysis (as part of combined effort by the consortium) can be well-powered to identify epigenetic associations.

Also, further programs to boost resilience in both civilians and military veterans can be of help as adjunct treatment of depression and PTSD, or in boosting resilience for prior known traumatic events, for example, prior to deployment in order to prevent PTSD in veterans going to combat zones.

Traumatic experiences violating a certain moral code or specific religious beliefs can be associated with moral injury, and possibly block recovery from PTSD and/or depression. Further 
allocation of resources to improve future research in areas related to resilience, the relationship between moral injury and PTSD, as well as the impact of religious involvement and other violations of moral values are warranted, as these help in fostering resilience and providing meaning to life.

Another important movement related to policy makers and society at large is improvement of education about the potential benefit of approved brain stimulation modalities such as Transcranial Magnetic Stimulation (TMS) and ECT. Although ECT is the most effective treatment in psychiatry (especially for treatment resistant mood disorder), it has bad publicity due to lack of education of the public, and erroneous media depictions of ECT as a method of torture rather than treatment. This can have deleterious effects on our patients and provide a serious barrier for care. In many cases, this results in avoiding a much-needed lifesaving treatment. Education campaigns about the role of ECT and these misconceptions, in addition to providing resources for studies to improve the cognitive side effects of ECT, would prove very helpful. Another application for this thesis is the use of both medications and ECT, rather than either alone, for maintenance of treatment-resistant patients with depression. We have shown in both a review of the literature, as well as in the analysis of the Prolonged Remission in Depressed Elderly (PRIDE) study, that the combination of ECT with medications helps patients to stay well in remission and also to improve quality of life and overall net health benefits compared with medications alone in treatment-resistant depression.

In order to mitigate the cognitive side effects of ECT that can discourage many patients indicated for ECT from receiving it, we presented a novel and promising technique of ECT named Low Pulse Amplitude Seizure Therapy (LAP-ST). We have presented a proof of concept clinical trial showing it to be feasible, safe, and with minimal or no cognitive side effects compared to the standard ECT techniques. We followed also with another pilot randomized clinical trial (not included in this thesis) using low amplitude-titrated seizure therapy, which was similarly promising (Youssef et al., 2019). A replication with a larger clinical trial may confirm these results can have substantial benefits on patients in terms of minimizing or avoiding memory and cognitive side effects during such treatments.

Much work needs to be done, but we hope that this work does not only get us a bit closer to advancing these fields, but also sheds some light on the important questions in these fields with widespread implication in helping patients and the society at large.

\section{Dissemination towards implementation:}

All the studies presented in this thesis have been submitted and are now published in peer-reviewed journals to help dissemination and implementation by clinicians, educators and policy makers. In addition, many of these studies have been presented as abstracts, posters and/or oral presentations in targeted scientific meetings for researchers, and others for clinicians. Some were presented as oral presentations to patient advocacy groups. Some studies also motivated media requests for interviews, and the information was disseminated by the interviews with journalists and presented in newspaper articles about the research and its findings, as well as radio and TV interviews locally, nationally, and internationally in an effort to speed the knowledge dissemination and subsequent implementation (including directions on how it can be implemented). These efforts would benefit the largest number of patients possible, help direct policy, and help decrease stigma of mental illness and of certain treatment modalities. 
*Parts adopted from Feng T, Youssef NA. Can epigenetic biomarkers lead us to precision medicine in predicting treatment response and remission for patients being considered for ECT? Psychiatry Res. 2019 Oct 28:112659. doi: 10.1016/j.psychres.2019.112659. [Epub ahead of print] PubMed PMID: 31703983. 


\section{REFERENCES}

American Psychiatric Association. (2013). Diagnostic and statistical manual of mental disorders (5th ed.). (Vol. 5th).

de Jong, J. O., Arts, B., Boks, M. P., Sienaert, P., van den Hove, D. L., Kenis, G., . . Rutten, B. P. (2014). Epigenetic effects of electroconvulsive seizures. J ect, 30(2), 152-159. doi:10.1097/yct.0000000000000141

Department of Veterans Affairs and Department of Defense. (2017). VA/DOD CLINICAL PRACTICE GUIDELINE FOR THE MANAGEMENT OF POSTTRAUMATIC STRESS DISORDER AND ACUTE STRESS DISORDER.

Domschke, K., Tidow, N., Schwarte, K., Deckert, J., Lesch, K. P., Arolt, V., . . Baune, B. T. (2014). Serotonin transporter gene hypomethylation predicts impaired antidepressant treatment response. Int J Neuropsychopharmacol, 17(8), 1167-1176. doi:10.1017/s146114571400039x

European Medicines Agency. (2013). Guideline on clinical investigation of medicinal products in the treatment of depression. Retrieved from Available from: http://www.ema.europa.eu/docs/en GB/document library/Scientific guideline/2013/0 5/WC500143770.pdf

Feng, T., \& Youssef, N. A. (2019). Can epigenetic biomarkers lead us to precision medicine in predicting treatment response and remission for patients being considered for ECT? Psychiatry Res, 112659. doi:10.1016/j.psychres.2019.112659

Goud Alladi, C., Etain, B., Bellivier, F., \& Marie-Claire, C. (2018). DNA Methylation as a Biomarker of Treatment Response Variability in Serious Mental Illnesses: A Systematic Review Focused on Bipolar Disorder, Schizophrenia, and Major Depressive Disorder. Int J Mol Sci, 19(10). doi:10.3390/ijms19103026

Kessler, R. C., Chiu, W. T., Demler, O., Merikangas, K. R., \& Walters, E. E. (2005). Prevalence, severity, and comorbidity of 12-month DSM-IV disorders in the National Comorbidity Survey Replication. Archives of general psychiatry, 62(6), 617-627. doi:10.1001/archpsyc.62.6.617

Kleimann, A., Kotsiari, A., Sperling, W., Groschl, M., Heberlein, A., Kahl, K. G., . . Frieling, H. (2015). BDNF serum levels and promoter methylation of BDNF exon I, IV and $\mathrm{VI}$ in depressed patients receiving electroconvulsive therapy. J Neural Transm (Vienna), 122(6), 925-928. doi:10.1007/s00702-014-1336-6

Kular, L., \& Kular, S. (2018). Epigenetics applied to psychiatry: Clinical opportunities and future challenges. Psychiatry Clin Neurosci, 72(4), 195-211. doi:10.1111/pcn.12634

Lisanby, S. H. (2007). Electroconvulsive therapy for depression. N Engl J Med, 357(19), 1939-1945. doi:10.1056/NEJMct075234

Miao, X.-R., Chen, Q.-B., Wei, K., Tao, K.-M., \& Lu, Z.-J. (2018). Posttraumatic stress disorder: from diagnosis to prevention. Military Medical Research, 5(1), 32-32. doi:10.1186/s40779-0180179-0 
Neyazi, A., Theilmann, W., Brandt, C., Rantamaki, T., Matsui, N., Rhein, M., .. . Loscher, W. (2018). P11 promoter methylation predicts the antidepressant effect of electroconvulsive therapy. Transl Psychiatry, 8(1), 25. doi:10.1038/s41398-017-0077-3

Powell, T. R., Smith, R. G., Hackinger, S., Schalkwyk, L. C., Uher, R., McGuffin, P., . . Tansey, K. E. (2013). DNA methylation in interleukin-11 predicts clinical response to antidepressants in GENDEP. Transl Psychiatry, 3, e300. doi:10.1038/tp.2013.73

Ratanatharathorn, A., Boks, M. P., Maihofer, A. X., Aiello, A. E., Amstadter, A. B., Ashley-Koch, A. E., . . . Smith, A. K. (2017). Epigenome-wide association of PTSD from heterogeneous cohorts with a common multi-site analysis pipeline. Am J Med Genet B Neuropsychiatr Genet, 174(6), 619-630. doi:10.1002/ajmg.b.32568

Rush, A. J., Trivedi, M. H., Wisniewski, S. R., Nierenberg, A. A., Stewart, J. W., Warden, D., . . . Fava, M. (2006). Acute and longer-term outcomes in depressed outpatients requiring one or several treatment steps: a STAR*D report. Am J Psychiatry, 163(11), 1905-1917. doi:10.1176/ajp.2006.163.11.1905

Sartor, C. E., McCutcheon, V. V., Pommer, N. E., Nelson, E. C., Grant, J. D., Duncan, A. E., ... Heath, A. C. (2011). Common genetic and environmental contributions to post-traumatic stress disorder and alcohol dependence in young women. Psychol Med, 41(7), 1497-1505. doi:10.1017/S0033291710002072

Stein, M. B., Jang, K. L., Taylor, S., Vernon, P. A., \& Livesley, W. J. (2002). Genetic and environmental influences on trauma exposure and posttraumatic stress disorder symptoms: a twin study. Am J Psychiatry, 159(10), 1675-1681. Retrieved from http://www.ncbi.nlm.nih.gov/entrez/query.fcgi?cmd=Retrieve\&db=PubMed\&dopt=Cita tion\&list uids $=12359672$

U.S. Department of Health and Human Services. (2018). Major Depressive Disorder: Developing Drugs for Treatment (Guidance for Industry) Retrieved from Available from: https://www.fda.gov/downloads/Drugs/GuidanceComplianceRegulatoryInformation/Gu idances/UCM611259.pdf

Wang, P., Lv, Q., Mao, Y., Zhang, C., Bao, C., Sun, H., . . . Fang, Y. (2018). HTR1A/1B DNA methylation may predict escitalopram treatment response in depressed Chinese Han patients. J Affect Disord, 228, 222-228. doi:10.1016/j.jad.2017.12.010

Wankerl, M., Miller, R., Kirschbaum, C., Hennig, J., Stalder, T., \& Alexander, N. (2014). Effects of genetic and early environmental risk factors for depression on serotonin transporter expression and methylation profiles. Transl Psychiatry, 4, e402. doi:10.1038/tp.2014.37

World Health Organization. (2017). Depression and other common mental disorders: global health estimates. Retrieved from

Yao, Z., McCall, W. V., Essali, N., Wohl, E., Parker, C., Rosenquist, P. B., \& Youssef, N. A. (2019). Precision ECT for major depressive disorder: A review of clinical factors, laboratory, and physiologic biomarkers as predictors of response and remission. Personalized Medicine in Psychiatry, 17-18, 23-31. doi:https://doi.org/10.1016/i.pmip.2019.07.001 
Youssef, N. A., Lockwood, L., Su, S., Hao, G., \& Rutten, B. P. F. (2018). The Effects of Trauma, with or without PTSD, on the Transgenerational DNA Methylation Alterations in Human Offsprings. Brain Sci, 8(5). doi:10.3390/brainsci8050083

Youssef, N. A., Ravilla, D., Patel, C., Yassa, M., Sadek, R., Zhang, L. F., . . Rosenquist, P. B. (2019). Magnitude of Reduction and Speed of Remission of Suicidality for Low Amplitude Seizure Therapy (LAP-ST) Compared to Standard Right Unilateral Electroconvulsive Therapy: A Pilot Double-Blinded Randomized Clinical Trial. Brain Sci, 9(5). doi:10.3390/brainsci9050099 


\section{Summary}

Psychological adaptation or resilience reflects functional, cognitive and/or behavioral adjustment to contextual (environmental) changes that comprises a key factor in psychological well-being. Lack of (or dysfunctional) adaption/resilience might contribute to stress-related disorders such as mood disorders and Posttraumatic Stress Disorder (PTSD). Stress-related disorders are also good models to study the interaction between nature and nurture (as both have significant genetic-environmental (GXE) interaction). They can also help study undesirable changes in neuronal function, adaptability, and neuroplasticity to understand how to reverse them to produce remission of symptoms. At the molecular biological level, epigenetic mechanisms may have an important role in the response to rapid environmental change and adaptation.

This thesis focuses and converges on two promising and rapidly evolving areas of neuroscience and psychiatry. These areas are epigenetics and brain stimulation. These 2 evolving fields can help to modify neuronal function, adaptability, and neuroplasticity and to investigate underlying (biological) processes. Stimulation of the brain also reflects exogenously induced interventions and can be seen as an environmental exposure. Thus, the research compiled in this thesis can be divided in two main sections: 'Epigenetics and Resilience' and 'Brain Stimulation' with ECT as the prototype for the purpose of this thesis.

The first section of this thesis focuses on epigenetics as one possible molecular mechanism underlying risk and resilience to stress-related disorders. It starts by providing an overview of recent advances in epigenetics as biomarkers in depression and an overview on epigenetics and PTSD, especially the evolving transgenerational transmission of the effects of PTSD via epigenetic methylation. It then presents our studies showing that higher resilience (that also have an epigenetic/biological component) predicts lower suicidality at 3-year follow-up, as well as lower depression as shown in another study we conducted. It also examines racial differences on the effect of trauma on development of depression. In a third study, non-biological factors such as moral injury and spirituality were also examined in how it impacts and correlates with PTSD symptoms. Finally, it presents our group efforts of establishing epigenetic consortium in PTSD and pooling data to increase the sample size for well-powered more definitive studies.

The second section of this thesis focuses on electroconvulsive therapy (ECT). It starts by reviewing the best way to prevent relapse after induction of remission (using a clinically-indicated acute course of ECT) for depressive episode of mood disorders. It also reviews other indications for ECT, including the emerging evidence that ECT might be of help in treatment-resistant PTSD, and the future direction for ECT research. As a related inquiry to the common indication of ECT in treatment-resistant depression, it also presents our randomized multicenter clinical trial on the effects of continuation of ECT plus medications versus continuation of medication alone on how the combination has significantly better improvement on quality of life. Then, it discusses cutting-edge research in the field, including research into biomarkers and neuroplasticity related to ECT response.

Moreover, in order to find ways to avoid (or minimize) the cognitive side effects of ECT, we examine a novel and focal form of stimulation: Low Pulse Amplitude Seizure Therapy (LAP-ST) for treatment of depressive episodes. In this clinical trial, we specifically examine the feasibility and preliminary evidence that it has less cognitive adverse effects compared to standard ECT. Our proof of concept clinical trial showed that this technique is feasible, and initial data showed LAP-ST to be safe, and with minimal cognitive side effects compared to the standard ECT while retaining efficacy.

Much work needs to be done, but we hope that the work in this thesis gets us a few steps closer to advancing the fields of epigenetics and ECT. We also hope there will be more examination of the interaction of these fields as presented in the discussion in the last chapter of this thesis, where we present a plan for future steps of research in this direction. We then present an addendum chapter on valorization of this knowledge so as to benefit the society, stakeholders, policy makers, as well as, researchers and clinicians and the efforts made to disseminate this information. 


\section{Acknowledgements}

I highly appreciate the support and contributions of Professor Rutten, thesis promotor as well as those of Professor Sienaert, thesis co-promotor. I also thank the assessment and defense committees for their time and help. I thank the staff and colleagues at Maastricht University for their help and kindness. I am also very appreciative for my family and friends who endured with me during the PhD process as well as their feedback and encouragement.

This PhD journey and the thesis would have not been possible without all the generosity of all the above individuals, as well as that of my patients and my research participants who volunteered for the advancement of science, finding transformative treatments (and a future cures) and to whom I am greatly in dept; and from whom I learned a lot thought-out my medical career. 


\section{About the Author}

Nagy Youssef's medical training included medical school at Cairo University, Egypt; psychiatry residency at University of South Alabama (Mobile, AL, USA) and postgraduate Consultation-Liaison training at Yale University (New Haven, CT, USA). He also completed a 3year research fellowship at both Durham VA and Duke University (Durham, NC, USA) and electroconvulsive therapy (ECT) \& Transcranial Magnetic Stimulation (TMS) fellowship at Duke University. As a faculty at Duke University, he established and directed a Treatment-Resistant Mood Disorders Outpatient Clinic, supervised residents and medical students in the clinic, in the Duke ECT service, and during weekly ECT rounding. He was one of the ECT and TMS faculty and investigators in the Division of Brain Stimulation \& Neurophysiology at Duke. He also taught physicians in the Duke ECT fellowship program and TMS fellowship.

After moving to Augusta, GA, USA, and as a faculty at Medical College of Georgia (MCG), he served as the medical director of acute psychiatric services (psychiatry inpatient and the psychiatric emergency department) at the affiliated Veterans Affairs Medical Center, then developed and currently directs a Mood and Trauma Clinic at MCG, where he also supervises residents and students in the clinic and is an ECT and brain stimulation faculty as well as directs a student clerkship in Mood Disorders.

His primary research interests include mechanistic and therapeutic innovation for treatment-resistant mood disorders, PTSD, and suicide prevention. His methodology and interventions within these areas include: 1) multiple brain stimulation mechanistic probes and therapeutics, including ECT, Transcranial Magnetic Stimulation (TMS), Magnetic Seizure Therapy (MST), and Transcranial Direct Current Stimulation (tDCS), and 2) genomics research. His studies involve primary clinical research, but he has occasionally studied non-human primates and translated the findings to humans.

He has presented his research in over 150 scientific papers and published abstracts. Dr. Youssef has lectured nationally and internationally on many research findings. The research studies he was involved in has been supported by the Department of Veterans Affairs, National Institute of Health, several research foundations, academic institutions, and pharmaceutical and device industries.

His service to the scientific community locally and internationally includes being a member of the Research Ethics Committee for 5 years at the Medical College of Georgia and as a member on the editorial board of several scientific journals. 